2018-12-01

\title{
Normally Supportive Sublattices of Crystallographic Space Groups
}

Miles A. Clemens

Brigham Young University

Follow this and additional works at: https://scholarsarchive.byu.edu/etd

Part of the Physical Sciences and Mathematics Commons

\section{BYU ScholarsArchive Citation}

Clemens, Miles A., "Normally Supportive Sublattices of Crystallographic Space Groups" (2018). Theses and Dissertations. 7705.

https://scholarsarchive.byu.edu/etd/7705

This Thesis is brought to you for free and open access by BYU ScholarsArchive. It has been accepted for inclusion in Theses and Dissertations by an authorized administrator of BYU ScholarsArchive. For more information, please contact ellen_amatangelo@byu.edu. 
Normally Supportive Sublattices of Crystallographic Space Groups

Miles A. Clemens

A thesis submitted to the faculty of

Brigham Young University

in partial fulfillment of the requirements for the degree of

Master of Science

\author{
Branton Campbell, Chair \\ Gus Hart \\ John Colton
}

Department of Physics and Astronomy

Brigham Young University

Copyright () 2018 Miles A. Clemens

All Rights Reserved 


\author{
ABSTRACT \\ Normally Supportive Sublattices of Crystallographic Space Groups \\ Miles A. Clemens \\ Department of Physics and Astronomy, BYU \\ Master of Science
}

Normal subgroups can be thought of as the primary building blocks for decomposing mathematical groups into quotient groups. The properties of the resulting quotient groups are often used to determine properties of the group itself. This thesis considers normal subgroups of threedimensional crystallographic space groups that are themselves three-dimensional crystallographic space groups; for convenience, we refer to such a subgroup as a csg-normal subgroup. We identify practical restrictions on csg-normal subgroups that facilitate their tabulation. First, the point group of an csg-normal subgroup must be a normal subgroup of the crystallographic point group of the space group, which we refer to for convenience as a cpg-normal subgroup. For each of the cpgnormal subgroups, which are all well known, we identify the abstract quotient group. Secondly, we identify necessary conditions on the sublattice basis of any csg-normal subgroup, and tabulate the "normally supportive" sublattices that meet these conditions, where some tables are symbolic forms that represent infinite families of sublattices. For a given space group, every csg-normal subgroup must be an extension of such a normally supportive sublattice, though some normally supportive sublattices may not actually support such extensions.

Keywords: space groups, normal subgroups, crystallography 


\section{ACKNOWLEDGMENTS}

I would like to acknowledge Harold Stokes who provided the code that was used for calculating the action of a parent space group on the cosets of its subgroup, Stephen Humphries who greatly assisted in the derivation of the theoretical foundation of the problem and also provided the analyses of some of the more difficult extra conditions, Branton Campbell for advising this research, Gus Hart and Wiley Morgan for their helpful discussions on the $P_{G}$-invariant condition, and Mois Aroyo who directed us to Vojtěch Kopský's work, which was highly relevant to this research. Fi-

nally, I would like to thank my wife who has supported me through graduate school and has taken good care of our baby when I have been too tired. 


\section{Contents}

Table of Contents $\quad$ iv

List of Figures

1 Introduction $\quad 1$

1.1 Groups and Crystal-Symmetry Groups . . . . . . . . . . . . . . . . . 1

1.2 A Simple Example . . . . . . . . . . . . . . . . . . . . 3

1.3 Affine Matrix Representation of Space-Group Elements . . . . . . . . . . . . . . . . 4

1.4 Applications of Normal Subgroups of Space Groups . . . . . . . . . . . . . . . 5

1.5 Goals and Strategy . . . . . . . . . . . . . . . . . . . 6

1.6 Motivating Example: Domain Structure of $\mathrm{YMnO}_{3} \ldots \ldots \ldots$

2 Point Groups $\quad 11$

2.1 Normal Subgroups and Quotients of Crystallographic Point Groups . . . . . . . 11

3 Normally Supportive Sublattices $\quad 15$

3.1 The Hermite Normal Form of a Sublattice Basis . . . . . . . . . . . . . . . . 15

3.2 Normally Supportive Conditions . . . . . . . . . . . . . . . . . . 16

3.3 Inclusion Sublattices . . . . . . . . . . . . . . . . . . . . 18

3.4 The Extension Problem . . . . . . . . . . . . . . . . . . 24

4 Algorithm $\quad 26$

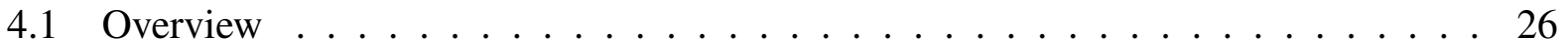

4.2 Subroutines . . . . . . . . . . . . . . . . . . 27

5 Results and Discussion $\quad 32$

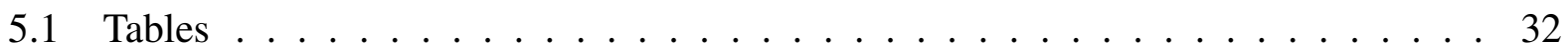

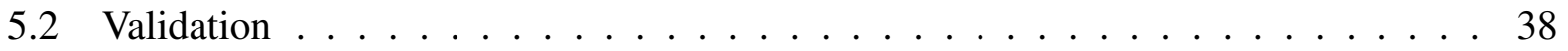

6 Examples 40

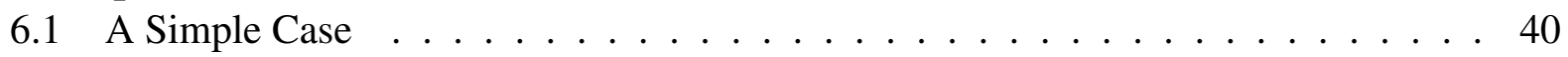

6.2 A Case with Bifurcation . . . . . . . . . . . . . . . . 43

6.3 A Case with Extra Conditions . . . . . . . . . . . . . . . 45 
6.4 A Very Messy Case . . . . . . . . . . . . . . . . . . . . . 48

7 Conclusions and Future Work $\quad 55$

$\begin{array}{ll}\text { Bibliography } & 58\end{array}$

$\begin{array}{lll}\text { Appendix A } & \text { Normally Supportive Sublattices } & 62\end{array}$ 


\section{List of Figures}

1.1 Dihedral Group . . . . . . . . . . . . . . . . . . . . . . . . 3

1.2 Commutative Diagram $\ldots \ldots \ldots \ldots \ldots \ldots$

1.3 Unit Cells of $\mathrm{YMnO}_{3}$ Domains $\ldots \ldots \ldots \ldots$

1.4 Topological Defect in $\mathrm{YMnO}_{3} \ldots \ldots \ldots \ldots$

1.5 Coset Graph of $\mathrm{YMnO}_{3}$ Domains $\ldots \ldots \ldots \ldots$ 


\section{List of Tables}

2.1 Normal Subgroups and Quotients of Point Groups . . . . . . . . . . . . . . . 13

2.2 Abstract Groups . . . . . . . . . . . . . . . . . . . . . 14

3.1 Inclusions . . . . . . . . . . . . . . . . . . . . . 19

5.1 Overview of Normally Supportive Sublattices . . . . . . . . . . . . . . . 33

A.1 Normally Supportive Sublattices . . . . . . . . . . . . . . . . . 62

A.2 Extra Conditions . . . . . . . . . . . . . . . . . 128 


\section{Chapter 1}

\section{Introduction}

\subsection{Groups and Crystal-Symmetry Groups}

A group, by definition, is a set with a binary operation with the following four properties: closure, associativity, an identity, and inverses for each element [1-4]. Closure means that the product of any two elements in the group will result in another element in the group (it is common to refer to the binary operation as multiplication and the result as the product). Associativity means that the order of operations is irrelevant, that is $a(b c)=(a b) c$ for any elements $a, b$, and $c$ in the group. The identity is an element (usually denoted as $e$ ) that leaves any other element invariant, that is to say $a e=a$ for any element $a$ in the group. The inverse of an element (usually denoted by -1 in the superscript) is the element that returns the identity when multiplied by the original element, that is to say $a^{-1} a=e$ for any element $a$ in the group.

We say that two elements $a, b \in G$ are conjugate elements if there exists some $g \in G$ such that $a=g^{-1} b g$. Any set of elements in $G$ that are all mutually conjugate is known as a conjugacy class. The set of all conjugacy classes of elements in $G$ partition the group, meaning that they are mutually disjoint and that their union is $G$. The identity is always alone in a conjugacy class since 
$g^{-1} e g=g^{-1} g=e$ for every $g \in G$.

A subgroup $H$ of $G$ is a subset of $G$ that possesses all of the properties that define a group under the same binary operation [1-4]. If $H$ is a subgroup of $G$, which we denote as $H \subseteq G$, then for any $a \in G$ we call the set $a H=\{a h \mid h \in H\}$ a coset of $H$ in $G$ containing $a$. If $N \subseteq G$ such that $a N=N a$ for every element $a \in G$ then we call $N$ a normal subgroup of $G$, which we denote as $N \unlhd G[1,2,4]$. A normal subgroup will only contain complete conjugacy classes of the parent, or in other words, either all of the elements in a conjugacy class are in the normal subgroup or none of them are. If $H \subseteq G$, then we call the largest subgroup of $H$ that is normal in $G$ the normal core of $H$ in $G$, and denote it as $N_{H}(G)$.

A normal subgroup $N \unlhd G$ is special in that the set of its cosets in $G$ form a group under the coset multiplication operation: $(a N)(b N)=a b N$ for any $a, b \in G$ (subgroups that are not normal do not have this property). This group of cosets is known as a quotient group (sometimes called a factor group) and is denoted by $G / N$ [1-4]. Coset multiplication is not well defined for a non-normal subgroup.

Group theory is relevant to the study of crystalline structures because the symmetries of a crystal form a group. A symmetry is any action on an object that leaves the object invariant. More generally, the symmetries of any physical system (e.g. an object, field, collection of objects and fields, model, or physical theory) form a group, wherein the group elements are transformations of the system and where the binary operation is composition. For objects like molecules and crystals, the relevant symmetries are geometric Euclidean isometries, which can be translations, point operations, or combinations of the two. A point symmetry leaves at least one point invariant. Examples are inversion, reflection about a plane, or rotation around a line. A translation symmetry translates the entire space by a fixed vector amount. The set of all point symmetries possessed by a crystal comprise a crystallographic point group [3]. The set of all symmetries possessed by a crystal comprise a crystallographic space group. A space group contains point symmetries, 
translational symmetries, and combinations of the two (glide planes and screw axes) [1,3]. The set of all pure translational symmetries possessed by a crystal comprise a crystallographic lattice group. This infinite lattice, whose rank is the dimension of the crystal, is a normal subgroup of the space group of the crystal. We will only consider three-dimensional crystals in the present work.

\subsection{A Simple Example}

To illustrate the concepts presented above, consider the six point symmetries of a $2 \mathrm{D}$ equilateral triangle, which form the dihedral group denoted as $D_{3}$. Let us define the composition $a b$ of geometric symmetries $a$ and $b$ such that the action of $b$ is applied to the object first, followed by the action of $a$.
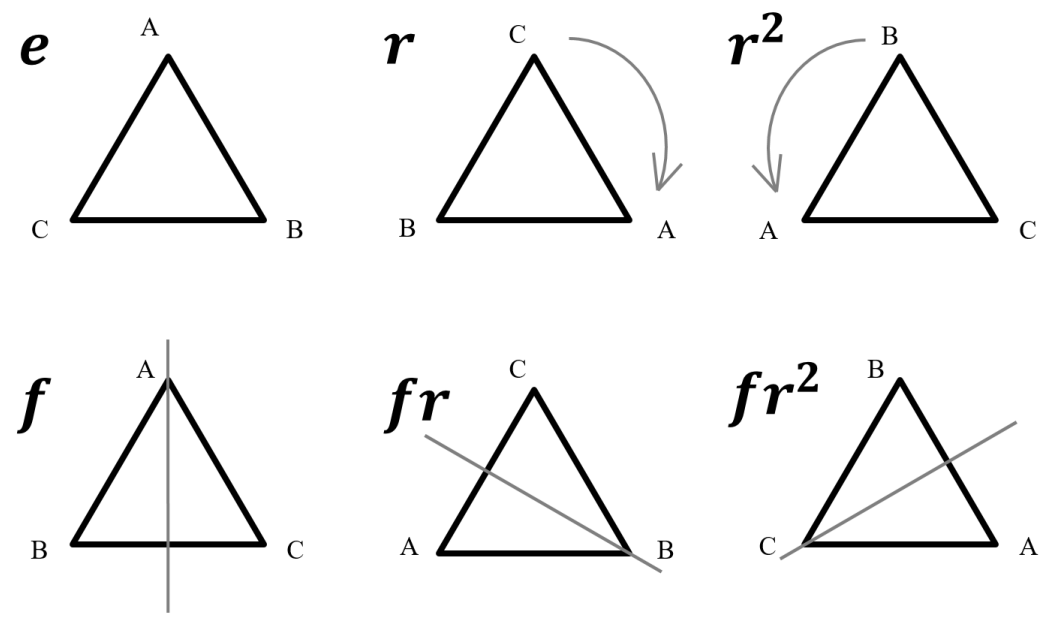

Figure 1.1 The symmetries of an equilateral triangle, commonly known as the dihedral group of order 6 and denoted as $D_{3}$.

The subgroups of $D_{3}$ are $\{e\},\{e, f\},\{e, f r\},\left\{e, f r^{2}\right\},\left\{e, r, r^{2}\right\}$, and $\left\{e, r, r^{2}, f, f r, f r^{2}\right\}$. Of these subgroups, both $\{e\}$ and $D_{3}$ are obviously normal subgroups. If we let $H=\left\{e, r, r^{2}\right\}$ then $H$ has two possible cosets, namely $e H=r H=r^{2} H=\left\{e, r, r^{2}\right\}$ and $f H=f r H=f r^{2} H=\left\{f, f r, f r^{2}\right\}$. Furthermore, we can see that $H e=H r=H r^{2}=\left\{e, r, r^{2}\right\}$ and $H f=H f r=H f r^{2}=\left\{f, f r, f r^{2}\right\}$. 
Thus for any element $a \in D_{3}, a H=H a$ so that $\left\{e, r, r^{2}\right\}$ is a normal subgroup of $D_{3}$. If we instead choose $H=\{e, f r\}$, the cosets of $H$ are $e H=f r H=\{e, f r\}, r H=f H=\{r, f\}$, and $r^{2} H=f r^{2} H=\left\{r^{2}, f r^{2}\right\}$. However, $H r=\left\{r, f r^{2}\right\} \neq r H$ so that $\{e, f r\}$ is not a normal subgroup of $D_{3}$. We see that $\{e\},\left\{e, r, r^{2}\right\}$, and $D_{3}$ are the only normal subgroups of $D_{3}$.

The cosets of the normal subgroup $N=\left\{e, r, r^{2}\right\}$ are $N=\left\{e, r, r^{2}\right\}$ and $f N=\left\{f, f r, f r^{2}\right\}$. Under coset multiplication, $(N)(N)=(f N)(f N)=\left\{e, r, r^{2}\right\}=N$ and $(N)(f N)=(f N)(N)=$ $\left\{f, f r, f r^{2}\right\}=f N$. Thus with $N$ as the identity, $\{N, f N\}$ is a group under the coset multiplication operation. In contrast, the cosets of non-normal subgroup $H=\{e, f r\}$ are $H=\{e, f r\}$, $r H=\{r, f\}$, and $r^{2} H=\left\{r^{2}, f r^{2}\right\}$, in which case $(r H)\left(r^{2} H\right)=\left\{e, r^{2}, f r, f r^{2}\right\}$ which is not one of the cosets of $H$. Because the cosets do not satisfy the closure condition, they do not form a group.

\subsection{Affine Matrix Representation of Space-Group Elements}

We find it convenient to use $4 \times 4$ affine-transformation matrices to represent the elements of a crystallographic space group, which allows us to employ matrix multiplication in performing symmetry-group calculations. The matrices are defined in the usual way relative to a basis of the lattice group of the crystal.

As an example, let $g$ be a $2_{1}$ screw axis in the $+z$ direction. The affine matrix representation of $g$ is

$$
g=\left(\begin{array}{cc}
p_{g} & t_{g} \\
0 & 1
\end{array}\right)=\left(\begin{array}{cccc}
-1 & 0 & 0 & 0 \\
0 & -1 & 0 & 0 \\
0 & 0 & 1 & \frac{1}{2} \\
0 & 0 & 0 & 1
\end{array}\right)
$$

where $p_{g}$ is a $3 \times 3$ matrix representing the point symmetry part and $t_{g}$ is a $3 \times 1$ translation vector. Every affine matrix has a $1 \times 3$ block of zeros on the bottom row followed by a 1 in the bottom right corner. 
The application of this affine matrix is the equivalent of applying the point operation first and then the translation. For example, the action of $g$ on coordinate $\chi=(x, y, z)$ is

$$
g \chi=p_{g}\left(\begin{array}{l}
x \\
y \\
z
\end{array}\right)+t_{g}=\left(\begin{array}{ccc}
-1 & 0 & 0 \\
0 & -1 & 0 \\
0 & 0 & 1
\end{array}\right)\left(\begin{array}{l}
x \\
y \\
z
\end{array}\right)+\left(\begin{array}{c}
0 \\
0 \\
\frac{1}{2}
\end{array}\right)=\left(\begin{array}{c}
-x \\
-y \\
z+\frac{1}{2}
\end{array}\right) .
$$

In other words, it will map the coordinate $(x, y, z)$ to $\left(-x,-y, z+\frac{1}{2}\right)$.

The International Tables for Crystallography presents representative space group elements (one for each coset of the translation subgroup) of each space group in terms of their action on coordinate $(x, y, z)$. The conversion of this action into affine matrix form is very straightforward.

\subsection{Applications of Normal Subgroups of Space Groups}

Normal subgroups and the corresponding quotients have seen a number of important applications in the context of crystallography. Let $H \subseteq G$ and let $K$ be the smallest subgroup of $G$ such that $H$ is a proper normal subgroup of $K ; K$ is then known as the normalizer of $H$ in $G$ [5]. Normalizers (Euclidean or affine) are commonly used for the identification of equivalent but symmetrically-unrelated Wyckoff orbits or crystal-structure coordinate descriptions [6-8]. The subperiodic groups (e.g. layer, rod, and frieze groups) of space groups have been systematically classified via their isomorphisms to space-group quotients $[9,10]$. Quotient groups of space groups with respect to normal subgroups are also employed in the construction of symmetry groups in higher than three dimensions via the use of subdirect products [11-13]. Subperiodic groups are associated via scanning tables with the symmetries of interfaces between crystal domains below a phase transition $[14,15]$. For a low-temperature phase transition giving rise to a ferroelectric space group (or ferromagntic space group) $H$ relative to a paraelectric (or paramagnetic) parent spacegroup $G$, the ferroic domains correspond to the cosets of $H$ in $G$, and the action of the normal core 
$N_{H}(G)$ on these cosets reveals the minimal symmetry of a multi-domain crystal with a spatially balanced domain configuration [16-18]. The ISOTROPY software program employs the action of the normal core to identify symmetry-unique domain-pair interfaces [19].

In 1987, Kopský laid out plans to identify and understand the normal subgroups of crystallographic space groups, and expected to follow his first article on this topic with a series of more specific results, though the subsequent articles never came to fruition. In that same article, he showed that only space groups that are irreducible (there is not a subgroup of the translation group that is left invariant the point group) have a finite number of csg-normal subgroups with nontrivial point groups [20]. A year later, it was reported that a table of normal subgroups of reducible space groups "require too much volume to be published in a journal" [21], which is a slightly odd statement given that the number of normal subgroups in such a case is actually infinite. In any case, the difficulty of the situation was clearly recognized. The present work is a key step towards an exhaustive table of three-dimensional normal subgroups of three-dimensional crystallographic space groups. One of our essential innovations has been the preparation of a finite table of infinite families of normally supportive sublattices in symbolic matrix form. Though Kopský and others clearly envisioned progress in this area, the computational tools that we used for symbolic algebraic reduction and manipulation were not available at that time.

\subsection{Goals and Strategy}

In the future, we hope to classify all csg-normal subgroups of each of the 230 crystallographic space groups, as well as the corresponding quotient groups. To accomplish this, our strategy is outlined as follows. Firstly, a space group $G$ is broken down into its point group, $P_{G}$, and its translation group, $T_{G}$. Secondly, all the normal subgroups of the point groups, $P_{N} \unlhd P_{G}$, are found individually. Thirdly, for each $P_{N}$, we must find the normal subgroups of the translation group, 
$T_{N} \unlhd T_{G}$, that support the normality of $T_{N} \rtimes P_{N}$ in $G$ (the symbol $\rtimes$ indicates a semidirect product); we explain what it means to support normality below in chapter $3[12,22]$. Fourthly, identify extensions $N$ of $P_{N}$ by $T_{N}$ such that $N \unlhd G$ (i.e. non-symmorphic space groups in the arithmetic crystal class of symmorphic space group $T_{N} \rtimes P_{N}$ ).

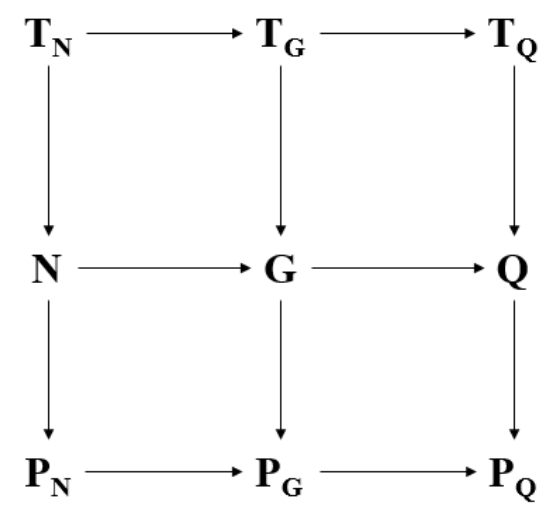

Figure 1.2 A commutative diagram consisting of short, exact sequences. The first arrow in any straight line points to a group from a normal subgroup of that group (e.g. $N \unlhd G$ ). The second arrow points to the quotient of the two groups (e.g. $Q=G / N$ ).

\subsection{Motivating Example: Domain Structure of $\mathrm{YMnO}_{3}$}

Here, we provide a motivating example that illustrates the use of csg-normal subgroups of a space group. Consider the domains of hexagonal yttrium manganite $\left(\mathrm{YMnO}_{3}\right) . \mathrm{YMnO}_{3}$ has a symmetry-lowering ferrielectric phase transition at $1258 \mathrm{~K}$ wherein atoms are displaced along the hexagonal $\mathrm{c}$ axis to form a low-symmetry hexagonal phase [23]. Below the transition temperature, there are six possible domains of the low temperature child structure which correspond to different directions of the ferroelectric and antiferroelectric order parameters [24]. The ferroelectric order parameter displaces all atoms of a given type in the same direction, whereas the antiferroelectric order parameter displaces some atoms of a given type in the $+z$ direction and the others in the $-z$ direction (see Figure 1.3) [25]. These six domains have been observed to meet at a one-dimensional 
topological defect, around which they form a six-fold pinwheel pattern (see Figure 1.4) [26, 27].
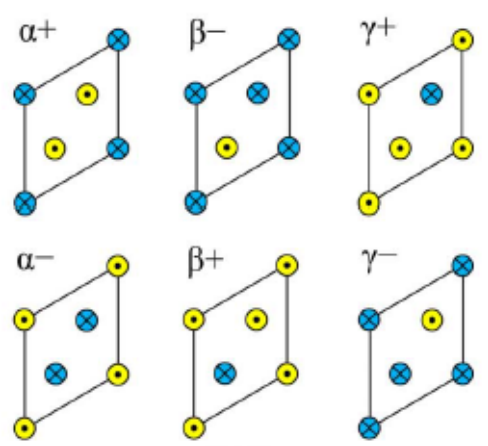

Figure 1.3 The displacements of yttrium atoms in the unit cells of each $\mathrm{YMnO}_{3}$ domain. [25]

Here, the space group $H$ of the low temperature phase $\left(P 6_{3} \mathrm{~cm}, \# 185\right)$ is a subgroup of the space group $G$ of the high temperature phase ( $P 6_{3} / m m c$, \#194); or in other words, $H \subseteq G$ [23]. However, because $H \not G$, the cosets of $H$ in $G$ do not form a quotient group, though the cosets of the normal core, $N_{H}(G)(P 3 c, \# 158)$, do result in a quotient group $Q=G / N_{H}(G)$. Because $Q$ is a group of cosets of $H$ in $G$, every element of $G$ acting on a coset is guaranteed to return another coset by closure. In other words, the action of $G$ permutes the elements of $Q$. This is physically meaningful because there is a one to one correspondence between the cosets of $H$ in $G$ and the physical domains of the $\mathrm{YMnO}_{3}$ crystal [28]. The elements of $G$ permute the elements of $Q$ in the same way the symmetries of the parent space group permute the low-temperature domains.

To visualize the physical significance of $Q$, consider Figure 1.3, where we focus attention on the three yttrium atoms in the unit cell. Notice that the $\beta^{+}$domain, if rotated 180 degrees about an axis parallel to the plane of the page, becomes the $\beta^{-}$domain. This rotation is a symmetry of the parent group which is not present in the child. Furthermore, the $\gamma^{+}$domain, if rotated along the exact same axis, becomes the $\gamma^{-}$domain. If a crystal had a domain boundary between the $\beta^{+}$ and $\gamma^{-}$domains, the boundary energy would have to be the same as that between the $\beta^{-}$and $\gamma^{+}$ domains because their boundaries are equivalent by symmetry. In general, if a pair of domains can 


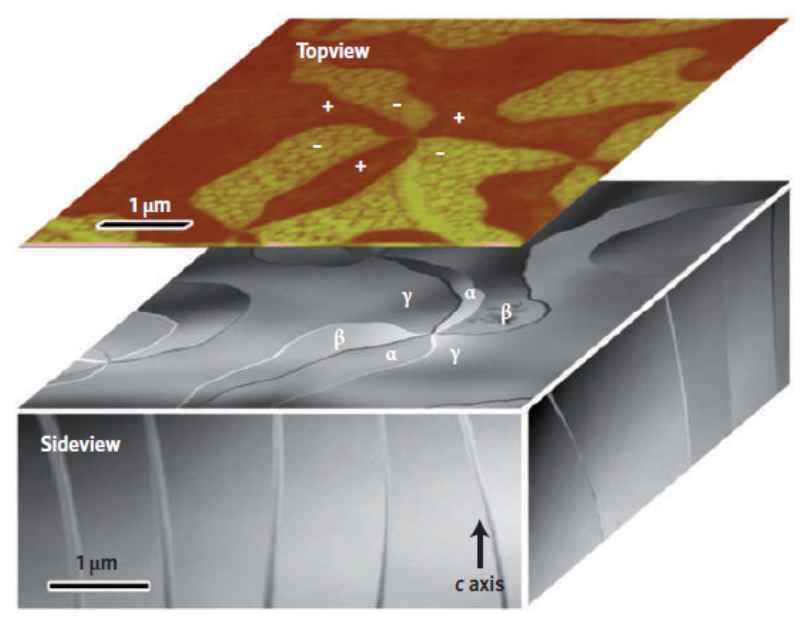

Figure 1.4 A ferroelectric map overlayed on an antiferroelectric map of $\mathrm{YMnO}_{3}$. This shows that all six domains come together to form topological defects. [27]

be transformed into another pair of domains by a symmetry operation of the parent group, then their boundary energies must be identical. The action of $G$ on the elements of $Q$ is transitive, so that any domain can be permuted into any other domain. However, this action is not transitive over domain pairs. Some domain pairs are not related to one another by any permutation enacted by $G$. Because not all pairs are symmetrically equivalent, we can group domain pairs into equivalence classes (Figure 1.5).

Using this idea, a graph of the domain boundary energies can be made to help visualize how the domains might meet in a crystal. Because $Q$ is isomorphic to three dimensional point group $6 \mathrm{~mm}$ (Schönflies $D_{6}$ or abstract $D_{3} \times \mathbb{Z}_{2}$ ), there is a natural way to embed the corresponding coset graph into the three-dimensional volume of the crystal. Assuming that the domain interfaces of one of the domain-pair equivalence classes will be energetically favorable relative to the others, we can select only the edges of that class from the coset graph to include in the embedding. In Figure 1.5, only the domain-pair equivalence class indicated by blue lines can yield the observed six-fold pinwheel pattern of Figure 1.4, wherein each domain favors interfaces with the two adjacement domains of 


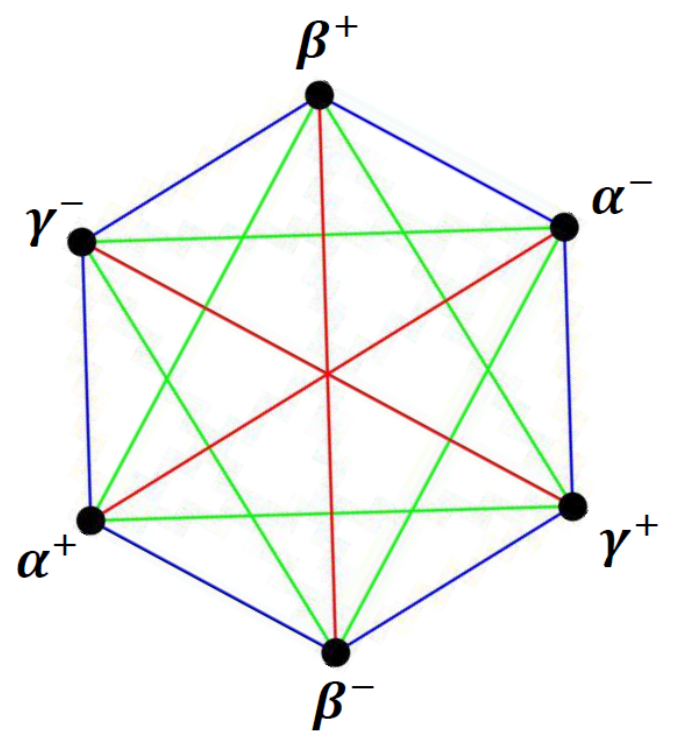

Figure 1.5 A domain graph of $\mathrm{YMnO}_{3}$. Each black vertex represents a domain and each connecting line between vertices represents a domain pair, where color indicates the domain-pair equivalence class.

the pinwheel. The green class breaks up into two subclasses of three domains each, either one of which would yield a three-fold pinwheel; and the red class breaks up into three subclasses of two domains, any of which would yield only single planar domain boundary.

We envision using this same approach to characterize domain-boundary topology in any crystal that exhibits symmetry-lowering phase transitions. For any $G$ and $H$ (both space groups), find the normal core, $N_{H}(G)$, and the quotient group, $G / N_{H}(G)$, generate the coset graph, and employ it to better understand the possible domain-interface topologies that can result. Hence the need to know the normal subgroups of crystallographic space groups, which have not been exhaustively studied in previous work. 


\section{Chapter 2}

\section{Point Groups}

\subsection{Normal Subgroups and Quotients of Crystallographic Point Groups}

The subgroups of each crystallographic point group are tabulated in the International Tables of Crystallography. [29] Our objective in this section is to identify each of the normal subgroups and to tabulate the corresponding quotient groups. That is, for a given subgroup $P_{H} \subseteq P_{G}$ we check to see if $g P_{H}=P_{H} g$ for every $g \in P_{G}$. If this is satisfied then $P_{H} \unlhd P_{G}$. Though not required for the derivation of normally supportive sublattices, point group quotients may prove to be useful in understanding the topology of defects in crystals. After finding the normal subgroups of a point group, it is not difficult to find the corresponding quotient group for each one.

Once we have tabulated every $P_{N} \unlhd P_{G}$, we can then tabulate every $P_{Q}=P_{G} / P_{N}$. To do this for a given $P_{G}$ and $P_{N}$, we first find the cosets of $P_{N}$ in $P_{G}$ by calculating $g P_{N}$ with every $g \in P_{G}$. This group of cosets under coset multiplication is $P_{Q}=P_{G} / P_{N}$. Table 2.1 lists each crystallographic point group $P_{G}$ and every cpg-normal subgroup $P_{N}$ with the corresponding quotient group $P_{Q}$. Though the contents of Table 2.1 have, no doubt, been derived in the past, we're not aware of a 
comparable presentation.

Since $P_{Q}$ is the group of cosets of $P_{N}$ in $P_{G}$, it is convenient to express $P_{Q}$ as an isomorphic abstract group. To do this, we see how each element of $P_{Q}$ permutes the elements of $P_{Q}$. We can then compare this permutation group to the permutation groups of the same order to find which groups are isomorphic. Table 2.2 lists the crystallographic point groups according to their isomorphism to abstract finite groups.

Tables 2.1 and 2.2 use symbols such as $\mathbb{Z}_{n}$ and $D_{n}$ to represent abstract groups. A brief explanation of each abstract group follows. $\mathbb{Z}_{n}$ represents the cyclic group of order $n$, which is isomorphic to the set of integers 0 through $n-1$ under addition modulo $n$. $D_{n}$ is the dihedral group of order $2 n$, which is isomorphic to the group of symmetries of a regular $n$-gon in two dimensions. $A_{n}$ is the alternating group of degree $n$, which is isomorphic to the set of even permutations of $n$ objects and has order $n ! / 2$. $S_{n}$ is the symmetric group of degree $n$, which is isomorphic to the set of all permutations of $n$ objects and has order $n !$. 
Table 2.1 A table of normal subgroups of crystallographic point groups and corresponding quotient groups. The first column is the crystallographic point group $P_{G}$, the second column is a crystallographic point group that is a normal subgroup $P_{N} \unlhd P_{G}$, the third column is an abstract group that is isomorphic to the quotient group $P_{Q}=P_{G} / P_{N}$. A subscript was added to the $P_{N}$ symbol where needed to clarify which crystallographic axis corresponds to the normal subgroup.

\begin{tabular}{|c|c|c|c|c|c|c|c|c|c|c|c|c|c|c|}
\hline$P_{G}$ & $P_{N}$ & $P_{Q}$ & $P_{G}$ & $P_{N}$ & $P_{Q}$ & $P_{G}$ & $P_{N}$ & $P_{Q}$ & $P_{G}$ & $P_{N}$ & $P_{Q}$ & $P_{G}$ & $P_{N}$ & $P_{Q}$ \\
\hline$\overline{1}$ & 1 & $\mathbb{Z}_{2}$ & $4 \mathrm{~mm}$ & 1 & $D_{4}$ & 321 & 1 & $D_{3}$ & 622 & 1 & $D_{6}$ & $6 / \mathrm{mmm}$ & $3 \mathrm{~m} 1$ & $D_{2}$ \\
\hline 2 & 1 & $\mathbb{Z}_{2}$ & $4 \mathrm{~mm}$ & 2 & $D_{2}$ & 321 & 3 & $\mathbb{Z}_{2}$ & 622 & $2 z$ & $D_{3}$ & $6 / \mathrm{mmm}$ & $31 \mathrm{~m}$ & $D_{2}$ \\
\hline $\mathrm{m}$ & 1 & $\mathbb{Z}_{2}$ & $4 \mathrm{~mm}$ & $\mathrm{~mm} 2$ & $\mathbb{Z}_{2}$ & $3 \mathrm{~m} 1$ & 1 & $D_{3}$ & 622 & 3 & $D_{2}$ & $6 / \mathrm{mmm}$ & $\overline{3} 1 \mathrm{~m}$ & $\mathbb{Z}_{2}$ \\
\hline $2 / \mathrm{m}$ & 1 & $D_{2}$ & $4 \mathrm{~mm}$ & 4 & $\mathbb{Z}_{2}$ & $3 \mathrm{~m} 1$ & 3 & $\mathbb{Z}_{2}$ & 622 & 312 & $\mathbb{Z}_{2}$ & $6 / \mathrm{mmm}$ & $\overline{3} \mathrm{~m} 1$ & $\mathbb{Z}_{2}$ \\
\hline $2 / \mathrm{m}$ & $\overline{1}$ & $\mathbb{Z}_{2}$ & $\overline{4} 2 \mathrm{~m}$ & 1 & $D_{4}$ & $31 \mathrm{~m}$ & 1 & $D_{3}$ & 622 & 321 & $\mathbb{Z}_{2}$ & $6 / \mathrm{mmm}$ & 6 & $D_{2}$ \\
\hline $2 / \mathrm{m}$ & 2 & $\mathbb{Z}_{2}$ & $\overline{4} 2 \mathrm{~m}$ & $2 z$ & $D_{2}$ & $31 \mathrm{~m}$ & 3 & $\mathbb{Z}_{2}$ & 622 & 6 & $\mathbb{Z}_{2}$ & $6 / \mathrm{mmm}$ & $\overline{6}$ & $D_{2}$ \\
\hline $2 / \mathrm{m}$ & $\mathrm{m}$ & $\mathbb{Z}_{2}$ & $\overline{4} 2 \mathrm{~m}$ & 222 & $\mathbb{Z}_{2}$ & $\overline{3} 1 \mathrm{~m}$ & 1 & $D_{6}$ & $6 \mathrm{~mm}$ & 1 & $D_{6}$ & $6 / \mathrm{mmm}$ & $6 / \mathrm{m}$ & $\mathbb{Z}_{2}$ \\
\hline 222 & 1 & $D_{2}$ & $\overline{4} 2 \mathrm{~m}$ & $\overline{4}$ & $\mathbb{Z}_{2}$ & $\overline{3} 1 \mathrm{~m}$ & $\overline{1}$ & $D_{3}$ & $6 \mathrm{~mm}$ & $2 z$ & $D_{3}$ & $6 / \mathrm{mmm}$ & 622 & $\mathbb{Z}_{2}$ \\
\hline 222 & 2 & $\mathbb{Z}_{2}$ & $\overline{4} \mathrm{~m} 2$ & 1 & $D_{4}$ & $\overline{3} 1 \mathrm{~m}$ & 3 & $D_{2}$ & $6 \mathrm{~mm}$ & 3 & $D_{2}$ & $6 / \mathrm{mmm}$ & $6 \mathrm{~mm}$ & $\mathbb{Z}_{2}$ \\
\hline $\mathrm{mm} 2$ & 1 & $D_{2}$ & $\overline{4} \mathrm{~m} 2$ & $2 z$ & $D_{2}$ & $\overline{3} 1 \mathrm{~m}$ & $\overline{3}$ & $\mathbb{Z}_{2}$ & $6 \mathrm{~mm}$ & $3 \mathrm{~m} 1$ & $\mathbb{Z}_{2}$ & $6 / \mathrm{mmm}$ & $\overline{6} \mathrm{~m} 2$ & $\mathbb{Z}_{2}$ \\
\hline $\mathrm{mm} 2$ & 2 & $\mathbb{Z}_{2}$ & $\overline{4} \mathrm{~m} 2$ & $\mathrm{~mm} 2$ & $\mathbb{Z}_{2}$ & $\overline{3} 1 \mathrm{~m}$ & 312 & $\mathbb{Z}_{2}$ & $6 \mathrm{~mm}$ & $31 \mathrm{~m}$ & $\mathbb{Z}_{2}$ & $6 / \mathrm{mmm}$ & $\overline{6} 2 \mathrm{~m}$ & $\mathbb{Z}_{2}$ \\
\hline $\mathrm{mmm}$ & 1 & $D_{2} \times \mathbb{Z}_{2}$ & $\overline{4} \mathrm{~m} 2$ & $\overline{4}$ & $\mathbb{Z}_{2}$ & $\overline{3} 1 \mathrm{~m}$ & $31 \mathrm{~m}$ & $\mathbb{Z}_{2}$ & $6 \mathrm{~mm}$ & 6 & $\mathbb{Z}_{2}$ & 23 & 1 & $A_{4}$ \\
\hline $\mathrm{mmm}$ & $\overline{1}$ & $D_{2}$ & $4 / \mathrm{mmm}$ & 1 & $D_{4} \times \mathbb{Z}_{2}$ & $\overline{3} \mathrm{~m} 1$ & 1 & $D_{6}$ & $\overline{6} \mathrm{~m} 2$ & 1 & $D_{6}$ & 23 & 222 & $\mathbb{Z}_{3}$ \\
\hline $\mathrm{mmm}$ & 2 & $D_{2}$ & $4 / \mathrm{mmm}$ & $\overline{1}$ & $D_{4}$ & $\overline{3} \mathrm{~m} 1$ & $\overline{1}$ & $D_{3}$ & $\overline{6} \mathrm{~m} 2$ & $\mathrm{~m}_{z}$ & $D_{3}$ & $\mathrm{~m} \overline{3}$ & 1 & $A_{4} \times \mathbb{Z}_{2}$ \\
\hline $\mathrm{mmm}$ & $\mathrm{m}$ & $D_{2}$ & $4 / \mathrm{mmm}$ & $2 z$ & $D_{2} \times \mathbb{Z}_{2}$ & $\overline{3} \mathrm{~m} 1$ & 3 & $D_{2}$ & $\overline{6} \mathrm{~m} 2$ & 3 & $D_{2}$ & $\mathrm{~m} \overline{3}$ & $\overline{1}$ & $A_{4}$ \\
\hline $\mathrm{mmm}$ & $2 / \mathrm{m}$ & $\mathbb{Z}_{2}$ & $4 / \mathrm{mmm}$ & $\mathrm{m}_{z}$ & $D_{4}$ & $\overline{3} \mathrm{~m} 1$ & $\overline{3}$ & $\mathbb{Z}_{2}$ & $6 \mathrm{~m} 2$ & 312 & $\mathbb{Z}_{2}$ & $\mathrm{~m} \overline{3}$ & 222 & $\mathbb{Z}_{6}$ \\
\hline $\mathrm{mmm}$ & 222 & $\mathbb{Z}_{2}$ & $4 / \mathrm{mmm}$ & $2 / \mathrm{m}_{z}$ & $D_{2}$ & $\overline{3} \mathrm{~m} 1$ & 321 & $\mathbb{Z}_{2}$ & $\overline{6} \mathrm{~m} 2$ & $3 \mathrm{~m} 1$ & $\mathbb{Z}_{2}$ & $\mathrm{~m} \overline{3}$ & $\mathrm{mmm}$ & $\mathbb{Z}_{3}$ \\
\hline $\mathrm{mmm}$ & $\mathrm{mm} 2$ & $\mathbb{Z}_{2}$ & $4 / \mathrm{mmm}$ & 222 & $D_{2}$ & $\overline{3} \mathrm{~m} 1$ & $3 \mathrm{~m} 1$ & $\mathbb{Z}_{2}$ & $6 \mathrm{~m} 2$ & $\overline{6}$ & $\mathbb{Z}_{2}$ & $\mathrm{~m} \overline{3}$ & 23 & $\mathbb{Z}_{2}$ \\
\hline 4 & 1 & $\mathbb{Z}_{4}$ & $4 / \mathrm{mmm}$ & $\mathrm{mm} 2_{z}$ & $D_{2}$ & 6 & 1 & $\mathbb{Z}_{6}$ & $\overline{6} 2 \mathrm{~m}$ & 1 & $D_{6}$ & 432 & 1 & $S_{4}$ \\
\hline 4 & 2 & $\mathbb{Z}_{2}$ & $4 / \mathrm{mmm}$ & $\mathrm{mmm}$ & $\mathbb{Z}_{2}$ & 6 & 2 & $\mathbb{Z}_{3}$ & $\overline{6} 2 \mathrm{~m}$ & $\mathrm{~m}_{z}$ & $D_{3}$ & 432 & 222 & $D_{3}$ \\
\hline$\overline{4}$ & 1 & $\mathbb{Z}_{4}$ & $4 / \mathrm{mmm}$ & 4 & $D_{2}$ & 6 & 3 & $\mathbb{Z}_{2}$ & $\overline{6} 2 \mathrm{~m}$ & 3 & $D_{2}$ & 432 & 23 & $\mathbb{Z}_{2}$ \\
\hline$\overline{4}$ & 2 & $\mathbb{Z}_{2}$ & $4 / \mathrm{mmm}$ & $\overline{4}$ & $D_{2}$ & $\overline{6}$ & 1 & $\mathbb{Z}_{6}$ & $\overline{6} 2 \mathrm{~m}$ & 321 & $\mathbb{Z}_{2}$ & $\overline{4} 3 \mathrm{~m}$ & 1 & $S_{4}$ \\
\hline $4 / \mathrm{m}$ & 1 & $\mathbb{Z}_{4} \times \mathbb{Z}_{2}$ & $4 / \mathrm{mmm}$ & $4 / \mathrm{m}$ & $\mathbb{Z}_{2}$ & $\overline{6}$ & $\mathrm{~m}$ & $\mathbb{Z}_{3}$ & $62 \mathrm{~m}$ & $31 \mathrm{~m}$ & $\mathbb{Z}_{2}$ & $\overline{4} 3 \mathrm{~m}$ & 222 & $D_{3}$ \\
\hline $4 / \mathrm{m}$ & $\overline{1}$ & $\mathbb{Z}_{4}$ & $4 / \mathrm{mmm}$ & 422 & $\mathbb{Z}_{2}$ & $\overline{6}$ & 3 & $\mathbb{Z}_{2}$ & $62 \mathrm{~m}$ & $\overline{6}$ & $\mathbb{Z}_{2}$ & $\overline{4} 3 \mathrm{~m}$ & 23 & $\mathbb{Z}_{2}$ \\
\hline $4 / \mathrm{m}$ & 2 & $D_{2}$ & $4 / \mathrm{mmm}$ & $4 \mathrm{~mm}$ & $\mathbb{Z}_{2}$ & $6 / \mathrm{m}$ & 1 & $\mathbb{Z}_{6} \times \mathbb{Z}_{2}$ & $6 / \mathrm{mmm}$ & 1 & $D_{6} \times \mathbb{Z}_{2}$ & $\mathrm{~m} \overline{3} \mathrm{~m}$ & 1 & $S_{4} \times \mathbb{Z}_{2}$ \\
\hline $4 / \mathrm{m}$ & $\mathrm{m}$ & $\mathbb{Z}_{4}$ & $4 / \mathrm{mmm}$ & $\overline{4} 2 \mathrm{~m}$ & $\mathbb{Z}_{2}$ & $6 / \mathrm{m}$ & $\overline{1}$ & $\mathbb{Z}_{6}$ & $6 / \mathrm{mmm}$ & $\overline{1}$ & $D_{6}$ & $\mathrm{~m} \overline{3} \mathrm{~m}$ & $\overline{1}$ & $S_{4}$ \\
\hline $4 / \mathrm{m}$ & $2 / \mathrm{m}$ & $\mathbb{Z}_{2}$ & $4 / \mathrm{mmm}$ & $\overline{4} \mathrm{~m} 2$ & $\mathbb{Z}_{2}$ & $6 / \mathrm{m}$ & 2 & $\mathbb{Z}_{6}$ & $6 / \mathrm{mmm}$ & $2 z$ & $D_{6}$ & $\mathrm{~m} \overline{3} \mathrm{~m}$ & 222 & $D_{6}$ \\
\hline $4 / \mathrm{m}$ & 4 & $\mathbb{Z}_{2}$ & 3 & 1 & $\mathbb{Z}_{3}$ & $6 / \mathrm{m}$ & $\mathrm{m}$ & $\mathbb{Z}_{6}$ & $6 / \mathrm{mmm}$ & $\mathrm{m}_{z}$ & $D_{6}$ & $\mathrm{~m} \overline{3} \mathrm{~m}$ & $\mathrm{mmm}$ & $D_{3}$ \\
\hline $4 / \mathrm{m}$ & $\overline{4}$ & $\mathbb{Z}_{2}$ & $\overline{3}$ & 1 & $\mathbb{Z}_{6}$ & $6 / \mathrm{m}$ & $2 / \mathrm{m}$ & $\mathbb{Z}_{3}$ & $6 / \mathrm{mmm}$ & $2 / \mathrm{m}_{z}$ & $D_{3}$ & $\mathrm{~m} \overline{3} \mathrm{~m}$ & 23 & $D_{2}$ \\
\hline 422 & 1 & $D_{4}$ & $\overline{3}$ & $\overline{1}$ & $\mathbb{Z}_{3}$ & $6 / \mathrm{m}$ & 3 & $D_{2}$ & $6 / \mathrm{mmm}$ & 3 & $D_{2} \times \mathbb{Z}_{2}$ & $\mathrm{~m} \overline{3} \mathrm{~m}$ & $\mathrm{~m} \overline{3}$ & $\mathbb{Z}_{2}$ \\
\hline 422 & $2 z$ & $D_{2}$ & $\overline{3}$ & 3 & $\mathbb{Z}_{2}$ & $6 / \mathrm{m}$ & $\overline{3}$ & $\mathbb{Z}_{2}$ & $6 / \mathrm{mmm}$ & $\overline{3}$ & $D_{2}$ & $\mathrm{~m} \overline{3} \mathrm{~m}$ & 432 & $\mathbb{Z}_{2}$ \\
\hline 422 & 222 & $\mathbb{Z}_{2}$ & 312 & 1 & $D_{3}$ & $6 / \mathrm{m}$ & 6 & $\mathbb{Z}_{2}$ & $6 / \mathrm{mmm}$ & 312 & $D_{2}$ & $\mathrm{~m} \overline{3} \mathrm{~m}$ & $\overline{4} 3 \mathrm{~m}$ & $\mathbb{Z}_{2}$ \\
\hline 422 & 4 & $\mathbb{Z}_{2}$ & 312 & 3 & $\mathbb{Z}_{2}$ & $6 / \mathrm{m}$ & $\overline{6}$ & $\mathbb{Z}_{2}$ & $6 / \mathrm{mmm}$ & 321 & $D_{2}$ & & & \\
\hline
\end{tabular}


Table 2.2 A table of abstract groups and all the crystallographic point groups that are isomorphic to each.

\begin{tabular}{|c|c|c|}
\hline Order & Abstract Groups & Isomorphic Point Groups \\
\hline 1 & $\mathbb{Z}_{1}$ & 1 \\
\hline 2 & $\mathbb{Z}_{2} \cong D_{1}$ & $\overline{1} \cong 2 \cong \mathrm{m}$ \\
\hline 3 & $\mathbb{Z}_{3}$ & 3 \\
\hline 4 & $D_{2} \cong \mathbb{Z}_{2} \times \mathbb{Z}_{2}$ & $222 \cong \mathrm{mm} 2 \cong 2 / \mathrm{m}$ \\
\hline 4 & $\mathbb{Z}_{4}$ & $4 \cong \overline{4}$ \\
\hline 6 & $D_{3}$ & $312 \cong 31 \mathrm{~m}$ \\
\hline 6 & $\mathbb{Z}_{6} \cong \mathbb{Z}_{3} \times \mathbb{Z}_{2}$ & $\overline{3} \cong 6 \cong \overline{6}$ \\
\hline 8 & $D_{2} \times \mathbb{Z}_{2} \cong \mathbb{Z}_{2} \times \mathbb{Z}_{2} \times \mathbb{Z}_{2}$ & $\mathrm{mmm}$ \\
\hline 8 & $D_{4}$ & $422 \cong 4 \mathrm{~mm} \cong \overline{4} 2 \mathrm{~m}$ \\
\hline 8 & $\mathbb{Z}_{4} \times \mathbb{Z}_{2}$ & $4 / \mathrm{m}$ \\
\hline 12 & $D_{6} \cong D_{3} \times \mathbb{Z}_{2}$ & $\overline{3} 1 \mathrm{~m} \cong 622 \cong 6 \mathrm{~mm} \cong \overline{6} \mathrm{~m} 2$ \\
\hline 12 & $A_{4}$ & 23 \\
\hline 12 & $\mathbb{Z}_{6} \times \mathbb{Z}_{2}$ & $6 / \mathrm{m}$ \\
\hline 16 & $D_{4} \times \mathbb{Z}_{2}$ & $4 / \mathrm{mmm}$ \\
\hline 24 & $A_{4} \times \mathbb{Z}_{2}$ & $\mathrm{~m} \overline{3}$ \\
\hline 24 & $S_{4}$ & $432 \cong \overline{4} 3 \mathrm{~m}$ \\
\hline 24 & $D_{6} \times \mathbb{Z}_{2}$ & $6 / \mathrm{mmm}$ \\
\hline 48 & $S_{4} \times \mathbb{Z}_{2}$ & $\mathrm{~m} \overline{3} \mathrm{~m}$ \\
\hline
\end{tabular}




\section{Chapter 3}

\section{Normally Supportive Sublattices}

\subsection{The Hermite Normal Form of a Sublattice Basis}

All matrices and vectors presented in this work are defined in the primitive parent setting, where the elements of $T_{G}$ can be represented as elements of $\mathbb{Z}^{3}$ (the set of all integer triplets). Any sublattice $T_{N} \subseteq T_{G}$ can then be represented as an integer matrix in Hermite normal form:

$$
B_{N}=\left(\begin{array}{ccc}
B_{11} & 0 & 0 \\
B_{21} & B_{22} & 0 \\
B_{31} & B_{32} & B_{33}
\end{array}\right)
$$

where the columns of $B_{N}$ are basis vectors of the sublattice $T_{N}$, and $0<\left\{B_{11}, B_{22}, B_{33}\right\}, 0 \leq B_{21}<$ $B_{22}$, and $0 \leq\left\{B_{31}, B_{32}\right\}<B_{33}$. The sublattice $T_{N}$ is then the set of translations $\left\{B_{N} t_{g} \mid t_{g} \in \mathbb{Z}^{3}\right\}$.

There are an infinite number of possible bases that span any given lattice, however each set of basis vectors will reduce to the same Hermite normal form matrix. By searching for a representation of $T_{N}$ in Hermite normal form, we are guaranteeing that each unique solution will represent a unique $T_{N}$. 


\subsection{Normally Supportive Conditions}

For a given parent space group $G$, tabulating all of the possible normal subgroups of the point group $P_{N} \unlhd P_{G}$ is not difficult since $P_{G}$ is finite [29]. Because any translation group is abelian, all elements of $T_{G}$ commute, and any possible sublattice $T_{N}$ of $T_{G}$ will be a normal subgroup of $T_{G}$. However, a given $P_{N} \unlhd P_{G}$ and an arbitrary $T_{N} \unlhd T_{G}$ may not be compatible for the purpose of generating subgroups of $G$, let alone normal subgroups of $G$ [21]. Because of this, we need to apply some restrictions on the form that $T_{N}$ can take.

In the setting of the parent lattice, each space group element can be expressed as a block affine matrix in the form

$$
g=\left(\begin{array}{cc}
p_{g} & t_{g} \\
0 & 1
\end{array}\right)=\left(\begin{array}{cc}
p_{g} & f_{g}+u_{g}+v_{g} \\
0 & 1
\end{array}\right) \in G
$$

where $p_{g} \in P_{G}$ is a $3 \times 3$ matrix representing the point component and $t_{g}$ is a $3 \times 1$ vector representing the translational component. The translation can be broken into three parts. The first part is a sublattice translation $v_{g} \in T_{N}$. The second part is a parent lattice translation $u_{g} \in T_{G}$ that lies within the unit cell of the sublattice (i.e. within the parallelepiped formed by the column vectors of $B_{N}$ ). The third part is a fractional translation $f_{g}$ that accommodates glide planes and screw axes.

Let us assume that $N \unlhd G$ and see what restrictions on $T_{N}$ result. Since normality requires $g h g^{-1} \in N$ for every $h \in N$ and $g \in G$, let us consider a $g$ that is simply a translation of the parent lattice. For any $u_{g}+v_{g} \in T_{G}$ and any $p_{h} \in P_{N}$ it follows that

$$
\left(\begin{array}{cc}
E & u_{g}+v_{g} \\
0 & 1
\end{array}\right)\left(\begin{array}{cc}
p_{h} & t_{h} \\
0 & 1
\end{array}\right)\left(\begin{array}{cc}
E & -\left(u_{g}+v_{g}\right) \\
0 & 1
\end{array}\right)=\left(\begin{array}{cc}
p_{h} & -p_{h}\left(u_{g}+v_{g}\right)+t_{h}+u_{g}+v_{g} \\
0 & 1
\end{array}\right) \in N
$$

However since

$$
\left(\begin{array}{cc}
p_{h} & t_{h} \\
0 & 1
\end{array}\right) \in N
$$


it follows that $u_{g}+v_{g}-p_{h}\left(u_{g}+v_{g}\right) \in T_{N}$ which can be rearranged as

$$
\left(E-p_{h}\right)\left(u_{g}+v_{g}\right) \in T_{N}
$$

Furthermore for any $v_{h} \in T_{N}$, normality requires that

$$
\left(\begin{array}{cc}
p_{g} & t_{g} \\
0 & 1
\end{array}\right)\left(\begin{array}{cc}
E & v_{h} \\
0 & 1
\end{array}\right)\left(\begin{array}{cc}
p_{g}^{-1} & -p_{g}^{-1} t_{g} \\
0 & 1
\end{array}\right)=\left(\begin{array}{cc}
E & p_{g} v_{h} \\
0 & 1
\end{array}\right) \in N
$$

and thus

$$
p_{g} v_{h} \in T_{N}
$$

It's important to recognize that applying $B_{N}^{-1}$ to every $v_{h} \in T_{N}$ yields the set of all integer triplets: $\left\{B_{N}^{-1} v_{h} \mid v_{h} \in T_{N}\right\}=\mathbb{Z}^{3}$. Thus, we can multiply equation 3.5 on the left side by $B_{N}^{-1}$ to obtain

$$
B_{N}^{-1}\left(E-p_{h}\right)\left(u_{g}+v_{g}\right) \in \mathbb{Z}^{3} .
$$

Equation 3.8 holds for every possible translation $u_{g}+v_{g}$, which runs over all of $\mathbb{Z}^{3}$. Only an integer matrix can transform every triplet in $\mathbb{Z}^{3}$ to $\mathbb{Z}^{3}$. Thus, equation 3.8 requires that

$$
B_{N}^{-1}\left(E-p_{h}\right) \in \mathbb{Z}^{3 \times 3}
$$

where $\mathbb{Z}^{3 \times 3}$ is the set of all $3 \times 3$ integer matrices.

Similarly we can multiply equation 3.7 on the left by $B_{N}^{-1}$ to get $B_{N}^{-1} p_{g} v_{h} \in \mathbb{Z}^{3}$ or equivalently

$$
\left(B_{N}^{-1} p_{g} B_{N}\right)\left(B_{N}^{-1} v_{h}\right) \in \mathbb{Z}^{3}
$$

and since $B_{N}^{-1} v_{h}$ can be any integer triplet then we must have

$$
B_{N}^{-1} p_{g} B_{N} \in \mathbb{Z}^{3 \times 3}
$$

For convenience, we refer to this condition as $P_{G}$-invariance. To support the normality condition, the basis of the sublattice must satisfy both equation 3.9 and equation 3.11 for every $p_{h} \in P_{N}$ and every $p_{g} \in P_{G}$. 
The focus of the present thesis, for a given $G$ and $P_{N}$, is to identify all $B_{N}$ that satisfy both equations 3.9 and 3.11 simultaneously. When $B_{N}$ satisfies both equations, we refer to the corresponding sublattice $T_{N}$ as "normally supportive" because it is capable of supporting an extension $N$ of $P_{N}$ by $T_{N}$ that is normal in $G$. This doesn't necessarily mean that such a normal extension will exist, only that its existence is not prevented by equations 3.9 and 3.11 .

One might imagine solving equations 3.9 and 3.11 separately, and then taking the intersection of their solution spaces. However, equation 3.11 by itself generally results in a very difficult system of Diophantine equations. Thus, we first use equation 3.9, which is straightforward to apply, to narrow the candidate forms of $B_{N}$, and only then apply equation 3.11 to each candidate form in order to further restrict the solution space of $B_{N}$. In the special case that $P_{N}=\{e\}$, equation 3.9 becomes trivial, thus leaving us to solve equation 3.11 alone, which does not lend itself to the computational tools used in this work and which would be impractical to treat by hand for every crystallographic space group $G$. Instead, such a case can be handled by a numerical approach that identifies all normally supportive $B_{N}$ up to a fixed supercell (i.e. sublattice unit cell) volume rather than determining all of the possibilities. [30]

\subsection{Inclusion Sublattices}

Here, we introduce another way of thinking about equation 3.9. Consider the group commutator of a lattice translation element $t_{g} \in T_{G}$ and a point group element $p_{h} \in P_{N}$, which is calculated as

$$
\left(\begin{array}{ll}
E & t_{g} \\
0 & 1
\end{array}\right)\left(\begin{array}{cc}
p_{h} & 0 \\
0 & 1
\end{array}\right)\left(\begin{array}{cc}
E & -t_{g} \\
0 & 1
\end{array}\right)\left(\begin{array}{cc}
p_{h}^{-1} & 0 \\
0 & 1
\end{array}\right)=\left(\begin{array}{cc}
E & \left(E-p_{h}\right) t_{g} \\
0 & 1
\end{array}\right) .
$$

By the conditions of normality, $p_{h}$ is conjugated by $t_{g}$ into another element of $N$ whose point part is $p_{h}$, which is then right-multiplied by $p_{h}^{-1}$ to obtain a pure translation that must be included in $N$. Or in other words, $\left(E-p_{h}\right) t_{g} \in T_{N}$ which is equivalent to equation 3.9 when $t_{g}$ is cycled over 
all of $\mathbb{Z}^{3}$. However, the restrictions imposed on $B_{N}$ are the same if we merely cycle $t_{g}$ over the generators of $\mathbb{Z}^{3}$, which generators are just the columns of the identity matrix. Our alternative to equation 3.9 then reduces to the statement that every row vector of $E-p_{h}$ for each $p_{h} \in P_{N} m u s t$ be included in $T_{N}$. For this reason, we refer to these row vectors loosely as "inclusion vectors". In general, the set of all row vectors obtained from each of the matrices $E-p_{h}$ result in some redundancy; in our implementation, we employ lattice-basis reduction to obtain a minimal set of inclusion vectors, which form the basis of an "inclusion sublattice" $T_{N}^{\prime} \subseteq T_{N}$. The restrictions on $B_{N}$ are then precisely those that allow only those sublattices $T_{N}$ that contain $T_{N}^{\prime}$.

The inclusion sublattice depends only upon the form of the operators $p_{h} \in P_{N}$ in the primitive parent setting, which in turn depend upon the lattice-centering type of $G$. When we consider all of the possibilities, we find 129 unique combinations of a parent lattice-centering type and a cpg-normal subgroup. We applied equation 3.9 to each of these 129 combinations and found only 41 unique inclusion sublattices, as detailed in Table 3.1. Thus, for any choice of $G$ and $P_{N}$, the restrictions imposed on $B_{N}$ from equation 3.9 will be identified with one of the 41 inclusion sublattices in this table. The actual restriction is that $B_{N}^{-1} b \in \mathbb{Z}^{3}$ for each basis vector $b$ in the inclusion sublattice.

Table 3.1 A table of the unique inclusion sublattice bases. The first column is an integer index for convenience. The second column contains unique inclusion sublattice bases, where each row is a single basis vector. The third column lists each of the pairs of parent lattice-centering type and cpg-normal subgroup that share the same inclusion sublattice. The $39^{\text {th }}$ inclusion sublattice, for example, lists 15 of such pairs.

Inclusion sublattice $P_{N}$ with corresponding centering type

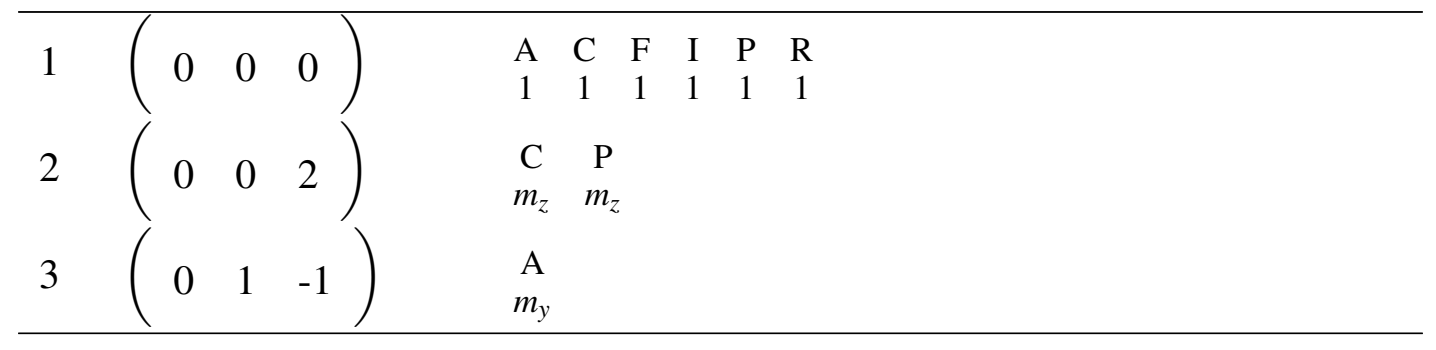


Table 3.1 - continued from previous page

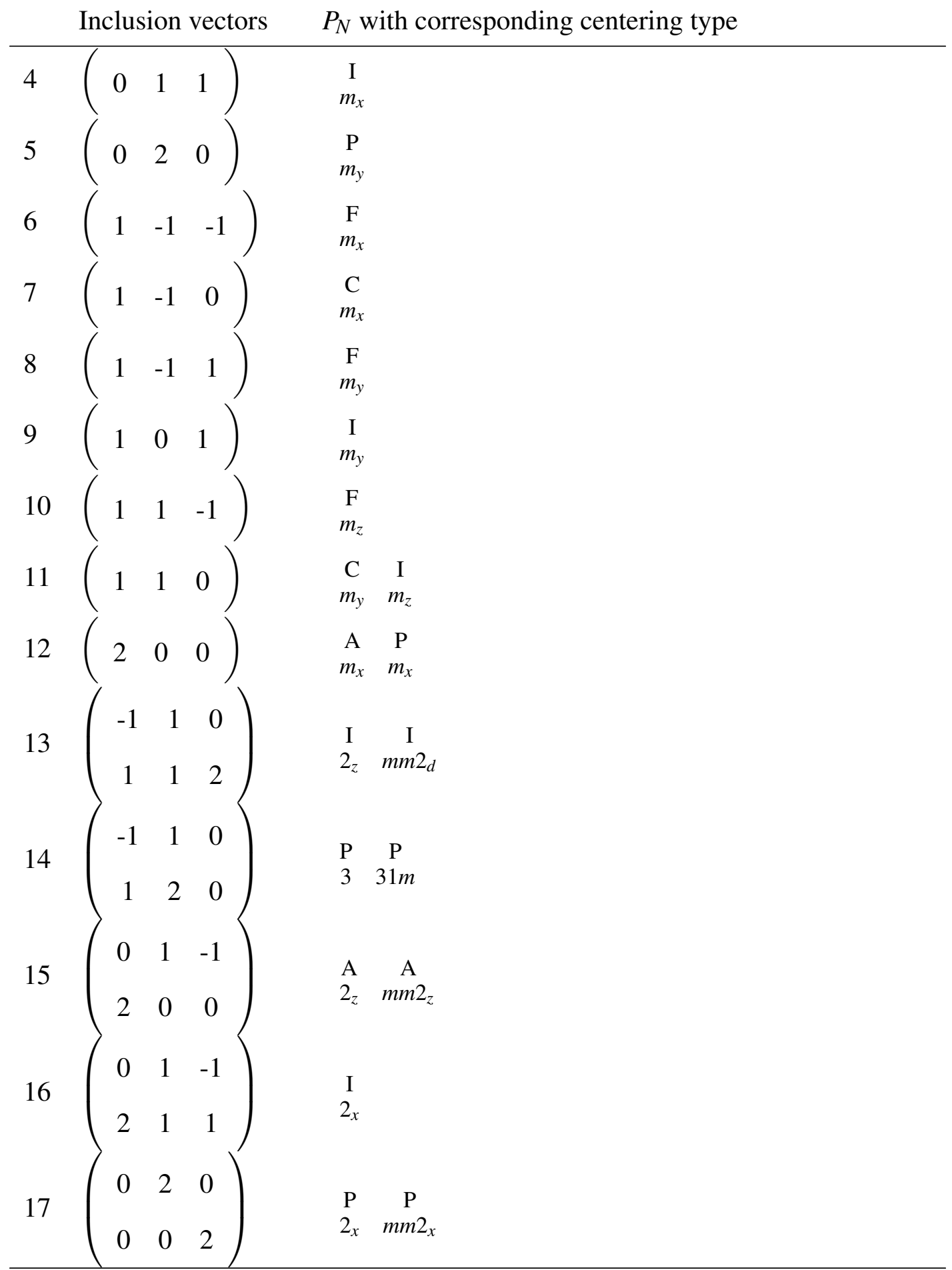


Table 3.1 - continued from previous page

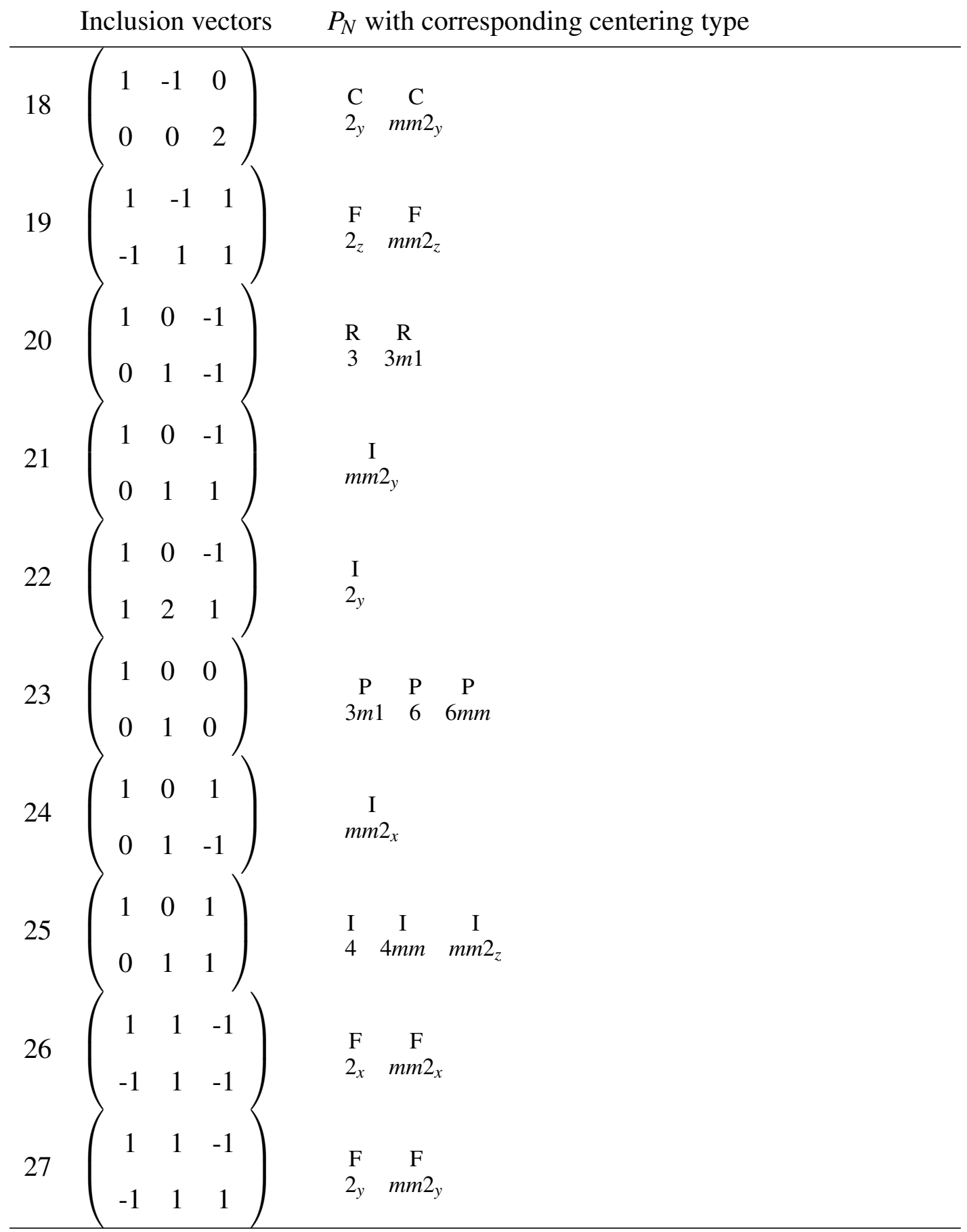


Table 3.1 - continued from previous page




Table 3.1 - continued from previous page

Inclusion vectors $\quad P_{N}$ with corresponding centering type

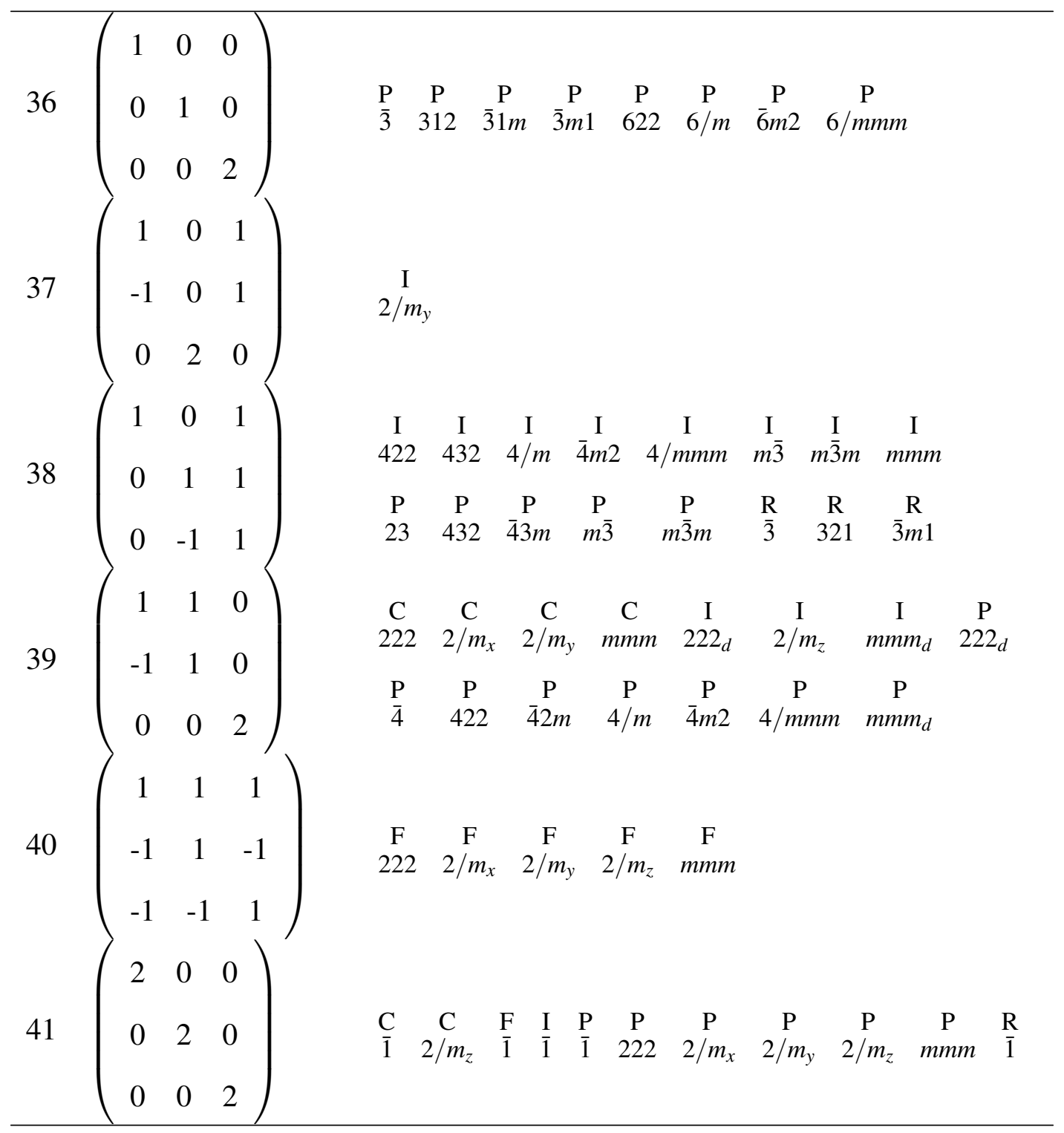




\subsection{The Extension Problem}

Because the fractional translation $f_{g}$ of each space group element is fixed by the choice of parent space group $G$, and the point operators $p_{h}$ are fixed by the choice of $P_{N}$, only the $u$ translations remain to be determined. Any set of $u$ translations that yields a valid subgroup (i.e. results in a set of operators that obey the same multiplication table as those of $P_{N}$ under multiplication modulo $T_{N}$ ) is an extension of $P_{N}$ by $T_{N}$ in $G$. Because the $u_{h}$ translation of any $p_{h}$ must be contained within the unit cell of the sublattice, there are only a finite number of ways to assign $u$ translations to each of the point operators, most of which will not result in subgroups. When a set of $u$ translations does yield a subgroup of $G$, that subgroup is a space group from the same arithmetic crystal class as $G$ (they are extensions of the same non-symmorphic group). In the remainder of this section, we will assume that the $u$ translations have already been restricted so that the extension $N$ of $P_{N}$ by $T_{N}$ is a valid subgroup of $G$.

In general, normality of $N$ in $G$ requires that $r=g h g^{-1} \in N$ for every $h \in N$ and $g \in G$. We can express this in affine matrix form as

$$
\left(\begin{array}{cc}
p_{r} & f_{r}+u_{r}+v_{r} \\
0 & 1
\end{array}\right)=\left(\begin{array}{cc}
p_{g} & f_{g}+u_{g}+v_{g} \\
0 & 1
\end{array}\right)\left(\begin{array}{cc}
p_{h} & f_{h}+u_{h}+v_{h} \\
0 & 1
\end{array}\right)\left(\begin{array}{cc}
p_{g}^{-1} & -p_{g}^{-1}\left(f_{g}+u_{g}+v_{g}\right) \\
0 & 1
\end{array}\right) .
$$

After expanding the product in equation 3.13, the point part reduces to

$$
p_{r}=p_{g} p_{h} p_{g}^{-1}
$$

and the translational part reduces to

$$
f_{r}+u_{r}+v_{r}=\left(E-p_{r}\right)\left(f_{g}+u_{g}+v_{g}\right)+p_{g}\left(f_{h}+u_{h}+v_{h}\right)
$$

Equation 3.14 merely requires that $P_{N} \unlhd P_{G}$, which is obvious. Since $v_{r} \in T_{N}$, we can rearrange equation 3.15 as

$$
v_{r}=\left[\left(E-p_{r}\right) f_{g}+p_{g}\left(f_{h}+u_{h}\right)-\left(f_{r}+u_{r}\right)\right]+\left(E-p_{r}\right)\left(u_{g}+v_{g}\right)+p_{g} v_{h} \in T_{N} .
$$


From equations $3.5,3.7$, and 3.16, it follows that

$$
\left(E-p_{r}\right) f_{g}+p_{g}\left(f_{h}+u_{h}\right)-\left(f_{r}+u_{r}\right) \in T_{N} .
$$

Equation 3.17 will be satisfied if and only if extension $N$ of $P_{N}$ by $T_{N}$ is normal in $G$. Again, we emphasize that for some normally supportive sublattices $T_{N}$ of $G$ with $P_{N}$, it may be that none of the extensions of $P_{N}$ by $T_{N}$ in $G$ will be normal in $G$. Thus some of the normally supportive sublattices obtained using the method described in the preceding sections may not actually support any normal extensions. But the sublattice of any csg-normal subgroup of $G$ must be normally supportive. Futhermore, because equations 3.9 and 3.11 do not involve the translational parts of the space-group operators, the normally-supportive sublattices $T_{N}$ with $P_{N}$ of two space groups $G_{1}$ and $G_{2}$ will be the same so long as $G_{1}$ and $G_{2}$ belong to the same arithmetic crystal class. 


\section{Chapter 4}

\section{Algorithm}

\subsection{Overview}

With a given point group $P_{G}$ and a normal subgroup $P_{N} \unlhd P_{G}$, the first step is to apply equation 3.9 with each $p_{h} \in P_{N}$ to find constraints on $B_{N}$. In general, each element of the matrix that results from the product on the left hand side of equation 3.9 can be a complicated rational expression containing all of the elements of $B_{N}$. Each of the matrix elements for each of the $p_{n} \in P_{N}$ must simultaneously take on integer values in order to satisfy the equation. We used Mathematica's built-in Reduce command to apply the integer restrictions and thereby find the values that the entries of $B_{N}$ can take.

Next, starting with the restricted form of $B_{N}$ resulting from equation 3.9, we do a similar thing with the much more difficult equation 3.11, where the elements of the matrix products for all $p_{g} \in P_{G}$ must simultaneously take on integer values. Of these terms, we are only interested in the ones that are not obviously integers. That is, we are interested in the terms with quotients. Again, we can use Mathematica's Reduce command to apply the integer-value restriction, and thereby obtain the restricted forms of $B_{N}$ that satisfy both equations 3.9 and 3.11. There are some quotient 
terms that are too complicated for Mathematica's built in reduction features. When this is the case, we simply keep track of these quotients and include the requirement that they be integral as extra conditions.

Because the normally supportive sublattices are identical for every space group in a given arithmetic crystal class, we organize our output according to the 73 arithmetic crystal classes, each of which is represented by its unique symmorphic space-group $G$. For a given $G$ and cpg-normal subgroup $P_{N}$, the output of our algorithm is a set of triplets, where the symmetry-restricted form of $B_{N}$ itself is the second part of each triplet. The first part of each triplet contains a list of inequalities that indicate the range of each variable in the corresponding $B_{N}$ matrix (this part is empty if there are no variables in $B_{N}$ ). The third part of each triplet contains any additional quotient expressions that must have integer values. In most cases, it was possible to achieve a standard form for the triplet in which the range of each variable is the set of all non-negative integers. The full set of triplets should collectively span the space of all normally supportive sublattices $T_{N}$ of $G$ with $P_{N}$. And all triplets should be mutually disjoint so as to entirely avoid redundancies.

\subsection{Subroutines}

The essential challenge with each of the many processing steps that we applied to the Mathematica's Reduce output was to modify the symbolic form of $B_{N}$ and its conditions without altering the solution space that they represent (i.e. the space of normally supportive sublattices). Thus every step of the processing yields a correct form but not necessarily a form that looks nice or is easy to understand. This effort required considerable attention to detail.

Depending on the specific case, the output from Reduce can take on a variety of different forms. A major component of the present research effort was devoted to bringing the output to a uniform format that follows relatively nice rules. First, simple steps were taken to extract information 
about the $B_{N}$ matrix and the conditions on its elements, eliminate special conditions that were obviously true, simplify complicated logical statements, and separate joint statements into lists of simultaneous conditions. A number of subroutines were then developed in order to further shape the output, each one handling a specific issue. We describe each subroutine below, where each paragraph begins with the name of the subroutine in quotes for easy reference to the Mathematica code.

The "relabelvars" module was used to relabel variables for convenience. To make each triplet look uniform, each variable is presented in the form of a letter combined with a number. Each variable that appears in an extra condition is denoted as $d_{n}$ while all the other variables are denoted as $c_{n}$ where $n$ is an integer. Each variable is then numbered in the order that it appears in the $B_{N}$ matrix, starting from the top and left side and proceeding row by row with the $c$ variables and $d$ variables numbered separately (i.e. a triplet can contain both $c_{1}$ and $d_{1}$ ). Because many of the subroutines introduce new variables into the triplets they modify, it was convenient to periodically relabel all of the variables in a triplet in a uniform way during the multi-step reduction process.

The "eliminatesimpleton" module was used to get rid of simple extra conditions. The extra conditions often took the form of an integer divided by a lone variable. To simplify this, we set the variable to a divisor of the numerator. Doing this can have multiple solutions when the numerator has multiple divisors. In such a case, the triplet bifurcates into multiple less complicated triplets, one for each solution.

The "removequotients" module was used to make the $B_{N}$ matrices look nicer. Sometimes the output of Reduce leaves quotients in the $B_{N}$ matrix. Since each $B_{N}$ must be in Hermite normal form, its entries must all be integers. For this reason, it was convenient to replace such a quotient with a new variable in $B_{N}$, and then solve for one of the other variables, adding the resulting quotient to the list of extra conditions in the last portion of the triplet.

The "cleanuptriplet" module was used to eliminate obvious extra conditions. Most of these 
subroutines aim to simplify the extra conditions, which sometimes leaves extra conditions that are unnecessary, redundant, or sometimes even impossible to satisfy. In the event that a triplet contains an extra condition that cannot possibly be an integer, such as $d_{2}+\frac{1}{2}$, it does not represent any possible normally supportive sublattices, and thus the entire triplet can be deleted. In the event that an extra condition is obviously an integer, e.g. $2, d_{1}+1,2 d_{2}\left(1-d_{2}\right)$, etc., we needn't keep track of it and thus it can be removed from the list of extra conditions. In the event that two extra conditions are linearly dependent, for example $\frac{1-d_{3}^{2}}{2}$ and $\frac{1-d_{3}^{2}}{2 d_{2}}$, the less restricting of the two $\left(\frac{1-d_{3}^{2}}{2}\right.$ in our example) is redundant and can be removed from the list of extra conditions. This module is implemented frequently because many of the other subroutines change the extra conditions so as to leave cases that can be cleaned up.

The "hyperreduce" module was used to simplify variable ranges. After removing the quotients from the $B_{N}$ matrices, some quotients are introduced into the conditions. This often tends to make the ranges on variables very complicated. To remedy this, we again use the Reduce command, but this time only on the obviously complicated conditions. This step sometimes has the side effect of making a single triplet split into multiple but nicer looking triplets.

The "eliminatefractional" module was used to simplify certain extra conditions. Sometimes there are extra conditions in the form $\frac{n_{1}}{n_{2}} d$ where $d$ is a variable in the $B_{N}$ matrix and $n_{1}$ and $n_{2}$ are integers. This can be simplified a little because the only values of $d$ that will satisfy this condition are multiples of $n_{2}$. If we simply let $h=d / n_{2}$, then we can replace all instances of $d$ in the triplet with $n_{2} h$ and thus we will have one less extra condition in these cases (the $h$ will then be relabeled as a new $c$ or $d$ variable).

The "replaceprettyuglies" module was used to simplify some of the nicer looking extra conditions. Sometimes there are extra conditions where a variable appears only once in the numerator of an extra condition. In other words, the extra condition has the form $\frac{\text { polynomial }_{1}+n d}{\text { polynomial }_{2}}$ where $n$ is a constant integer, $d$ is a variable in the $B_{N}$ matrix and the polynomials do not contain the variable $d$. 
This condition can be eliminated with a little work. If we let $\frac{\text { polynomial }_{1}+n d}{\text { polynomial }_{2}}=k$ then we can replace all instances of $d$ in the triplet with $\frac{1}{n}\left(\right.$ kpolynomial $_{2}-$ polynomial $\left._{1}\right)$ which will eliminate this extra condition ( $k$ will then be relabeled as a new $c$ or $d$ variable). The standard form doesn't allow any variable expression in $B_{N}$ that are not obviously integers. To avoid introducing numerical fractions in $B_{N}$ in this step, we make a second replacement to remove any $\frac{1}{n}$ that might appear in the triplet.

The "eliminatedependentvars" module was used to restrict the number of variables to a minimum. Sometimes the output of Reduce gives more variables than is necessary. For example, a particular $B_{N}$ matrix might have only three elements with variables which have the form $2\left(3+d_{1}+d_{2}+d_{3}\right), 2\left(4+d_{1}+2 d_{2}\right)$, and $4\left(3+d_{1}+d_{2}+d_{3}\right)$. By inspection it can be seen that the third element is exactly double the first element and thus there are only two linearly independent polynomials. This is a problem because there are three variables to span $B_{N}$ which means that there are redundancies. The desired format is by definition free of variable redundancies - it doesn't permit more than one set of variable values to achieve the same numerical $B_{N}$ matrix. To fix this problem, it was necessary to rewrite these polynomials in terms of only two variables that span the same $B_{N}$ space. To do this we implemented Mathematica's GroebnerBasis command which takes a series of polynomials and returns a set of linearly independent polynomials that span the same space. In the example above, the Gröbner basis is $\left\{d_{2}-d_{3}, d_{1}+2 d_{3}\right\}$. If we let $f_{1}=d_{2}-d_{3}$ and $f_{2}=d_{1}+2 d_{3}$ then we can replace every instance of $d_{1}$ with $f_{2}-2 d_{3}$ and every instance of $d_{2}$ with $f_{1}+d_{3}$ then the original expressions above become $2\left(3+f_{1}+f_{2}\right), 2\left(1+2 f_{1}+f_{2}\right)$, and $4\left(3+f_{1}+f_{2}\right)$ which has only two variables; the $f$ variables will then be relabeled as $c$ or $d$ variables.

The "removeexcesscondvars" module was used to get rid of unnecessary conditions on the ranges of the variables. While the eliminatedependentvars routine does an excellent job at removing unnecessary variables in the $B_{N}$ matrices, it fails to reduce the corresponding number of variables in the list of conditions. To illustrate this problem, consider the example in the previous 
paragraph where $d_{1}, d_{2}$, and $d_{3}$ could be any non-negative integer. After replacing $d_{1}$ with $f_{2}-2 d_{3}$ and $d_{2}$ with $f_{1}+d_{3}$, our conditions become $f_{2}-2 d_{3} \geq 0, f_{1}+d_{3} \geq 0$, and $d_{3} \geq 0$. It is no longer necessary to keep track of $d_{3}$ since it was eliminated from $B_{N}$, however $d_{3}$ is necessary to determine what values $f_{1}$ and $f_{2}$ can take. To eliminate $d_{3}$ from the conditions, we first isolate it in each inequality which $f_{2} / 2 \geq d_{3}, d_{3} \geq-f_{1}$, and $d_{3} \geq 0$. We can then combine these three conditions into the following two compound inequalities $f_{2} / 2 \geq d_{3} \geq 0$ and $f_{2} / 2 \geq d_{3} \geq-f_{1}$. Since the value of $d_{3}$ makes no difference in $B_{N}$, we can choose to set it equal to the shared maximum value in both compound inequalities which then simplifies them to $f_{2} / 2 \geq 0$ and $f_{2} / 2 \geq-f_{1}$ and thus $d_{3}$ is now completely removed from the triplet. This module has the unfortunate side effect of leaving somewhat awkward conditions, which are later improved by the hyperreduce subroutine.

The order in which the subroutines were applied is somewhat strategic and somewhat arbitrary. Often, the application of one subroutine recreated undesirable structures within the matrix or conditions that were resolved by a previous subroutine, so that the first subroutine needed to be reapplied. This led to seemingly arbitrary iterations in the application of certain groups of subroutines. The specific and rather delicate ordering of subroutine calls that we ultimately converged on did bring all cases into the desired uniform format; but it is likely that other orderings might also accomplish this. However, we also point out some small and seemingly unimportant deviations from the specific ordering that we applied failed spectacularly to deliver the desired format. 


\section{Chapter 5}

\section{Results and Discussion}

\subsection{Tables}

Normally supportive sublattices have been tabulated and are listed in appendix A. Many table entries contain a symbolic $B_{N}$ matrix that actually represents an infinite family of sublattices. Table 5.1 is a summary of Table A.1 organized by arithmetic crystal class. The first column is an integer index for the 73 arithmetic crystal classes. The second column is the symmorphic crytallographic space group symbol that represents the arithmetic class. The third column is the total number of $B_{N}$ matrices from all cpg-normal subgroups of the arithmetic class. The last column indicates the number of $B_{N}$ families in square brackets associated with each cpg-normal subgroup of an arithmetic class.

There are a total of $1773 B_{N}$ families accounted for in Table 5.1. Keep in mind that some families are larger than others due to differences in dimensionality (i.e. the number of free variables of infinite range in the symbolic form). For a given cpg-normal subgroup, one three-dimensional $B_{N}$ family is vastly larger than three zero-dimensional $B_{N}$ families, but collectively equivalent in size to three one-dimensional $B_{N}$ families. For example, ACC $R 3$ (\#39) lists only one cpg- 
normal subgroup, which has only a single $B_{N}$ family, which turns out to be a one-dimensional infinite family. ACC $P \overline{1}(\# 2)$, on the other hand, lists only one cpg-normal subgroup, which has 16 different $B_{N}$ families, all of which turn out to be zero-dimensional (i.e. families containing one individual each).

Due to the exceptional complexity of most cases for which the cpg-normal subgroup is 1 , all of these cases were excluded from the present work and tables, including the handful of cases that proved to be readily solvable using the present methods. As mentioned earlier, cases for which the npg-subgroup is 1 can be treated numerically by method of Morgan and Hart. [30]

Table 5.1 The number of families of normally-supportive sublattices for each arithmetic crystal class (ACC), and for each cpg-normal subgroup of each ACC.

\begin{tabular}{|c|c|c|c|c|c|}
\hline $\mathrm{ACC}$ & total & \multicolumn{4}{|c|}{ cpg-normal subgroups and number of corresponding $B_{N}$ families } \\
\hline $1 \mathrm{P} 1$ & - & - & & & \\
\hline $2 \mathrm{P} \overline{1}$ & 16 & $\overline{1}[16]$ & & & \\
\hline $3 \mathrm{P} 2$ & 11 & $2 z[11]$ & & & \\
\hline $4 \mathrm{C} 2$ & 11 & $2{ }_{z}[11]$ & & & \\
\hline $5 \mathrm{Pm}$ & 5 & $\mathrm{~m}_{z}[5]$ & & & \\
\hline $6 \mathrm{Cm}$ & 5 & $\mathrm{~m}_{z}[5]$ & & & \\
\hline $7 \mathrm{P} 2 / \mathrm{m}$ & 48 & $\overline{1}[16]$ & $2_{z}[11]$ & $\mathrm{m}_{z}[5]$ & $2 / \mathrm{m}_{z}[16]$ \\
\hline $8 \mathrm{C} 2 / \mathrm{m}$ & 48 & $\overline{1}[16]$ & $2_{z}[11]$ & $\mathrm{m}_{z}[5]$ & $2 / \mathrm{m}_{z}[16]$ \\
\hline 9 P222 & 49 & $2{ }_{x}[11]$ & $22_{y}[11]$ & $2{ }_{z}[11]$ & $222[16]$ \\
\hline $10 \mathrm{C} 222$ & 19 & $2_{x}[6]$ & $2 y[3]$ & $2_{z}[5]$ & $222[5]$ \\
\hline $11 \mathrm{~F} 222$ & 15 & $2 x_{x}[4]$ & $2 y[3]$ & $2 z[3]$ & $222[5]$ \\
\hline $12 \mathrm{I} 222$ & 21 & $2 x_{x}[5]$ & $2_{y}[5]$ & $2_{z}[5]$ & $222[6]$ \\
\hline 13 Pmm2 & 44 & $2{ }_{z}[11]$ & $\mathrm{m}_{x}[11]$ & $\mathrm{m}_{y}[11]$ & $\mathrm{mm} 2_{z}[11]$ \\
\hline $14 \mathrm{Cmm} 2$ & 14 & $2_{z}[5]$ & $\mathrm{m}_{x}[2]$ & $\mathrm{m}_{y}[4]$ & $\mathrm{mm} 2_{z}[3]$ \\
\hline $15 \mathrm{Amm} 2$ & 85 & $2 z[3]$ & $\mathrm{m}_{x}[77]$ & $\mathrm{m}_{y}[2]$ & $\mathrm{mm} 2_{z}[3]$ \\
\hline 16 Fmm2 & 19 & $2 z[3]$ & $\mathrm{m}_{x}[2]$ & $\mathrm{m}_{y}[11]$ & $\mathrm{mm} 2_{z}[3]$ \\
\hline $17 \mathrm{Imm} 2$ & 34 & $2{ }_{z}[5]$ & $\mathrm{m}_{x}[7]$ & $\mathrm{m}_{y}[20]$ & $\mathrm{mm} 2_{z}[2]$ \\
\hline
\end{tabular}


Table 5.1 - continued from previous page

\begin{tabular}{|c|c|c|c|c|c|c|c|c|}
\hline \multirow{2}{*}{$\begin{array}{l}\text { ACC } \\
18 \text { Pmmm }\end{array}$} & \multirow{2}{*}{$\begin{array}{l}\text { total } \\
192\end{array}$} & \multicolumn{7}{|c|}{ cpg-normal subgroups and number of corresponding $B_{N}$ families } \\
\hline & & $\overline{1}[16]$ & $22_{x}[11]$ & $2 y[11]$ & $2{ }_{z}[11]$ & $\mathrm{m}_{x}[11]$ & $\mathrm{m}_{y}[11]$ & $\mathrm{m}_{z}[8]$ \\
\hline \multirow{3}{*}{$19 \mathrm{Cmmm}$} & \multirow{3}{*}{77} & $\begin{array}{l}2 / \mathrm{m}_{x}[16] \\
\mathrm{mmm}[16]\end{array}$ & $2 / \mathrm{m}_{y}[16]$ & $2 / \mathrm{m}_{z}[16]$ & $222[16]$ & $\operatorname{mm} 2_{x}[11]$ & $\mathrm{mm} 2_{y}[11]$ & $\mathrm{mm} 2_{z}[11]$ \\
\hline & & $\overline{1}[8]$ & $2 x[6]$ & $2 y[3]$ & $2_{z}[5]$ & $\mathrm{m}_{x}[2]$ & $\mathrm{m}_{y}[4]$ & $\mathrm{m}_{z}[9]$ \\
\hline & & $\begin{array}{l}2 / \mathrm{m}_{x}[5] \\
\mathrm{mmm}[5]\end{array}$ & $2 / \mathrm{m}_{y}[5]$ & $2 / \mathrm{m}_{z}[8]$ & $222[5]$ & $\mathrm{mm} 2_{x}[6]$ & $\mathrm{mm} 22_{y}[3]$ & $\mathrm{mm} 2_{z}[3]$ \\
\hline \multirow[t]{2}{*}{$20 \mathrm{Fmmm}$} & \multirow[t]{2}{*}{79} & $\overline{1}[6]$ & $2 x[4]$ & $2 y[3]$ & $2_{z}[3]$ & $\mathrm{m}_{x}[2]$ & $\mathrm{m}_{y}[11]$ & $\mathrm{m}_{z}[15]$ \\
\hline & & $\begin{array}{l}2 / \mathrm{m}_{x}[5] \\
\mathrm{mmm}[5]\end{array}$ & $2 / \mathrm{m}_{y}[5]$ & $2 / \mathrm{m}_{z}[5]$ & $222[5]$ & $\mathrm{mm} 2_{x}[4]$ & $\mathrm{mm} 2_{y}[3]$ & $\mathrm{mm} 2_{z}[3]$ \\
\hline \multirow[t]{2}{*}{$21 \mathrm{Immm}$} & \multirow[t]{2}{*}{83} & $\overline{1}[6]$ & $22_{x}[5]$ & $22_{y}[5]$ & $2_{z}[5]$ & $\mathrm{m}_{x}[7]$ & $\mathrm{m}_{y}[20]$ & $\mathrm{m}_{z}[12]$ \\
\hline & & $\begin{array}{l}2 / \mathrm{m}_{x}[3] \\
\mathrm{mmm}[2]\end{array}$ & $2 / \mathrm{m}_{y}[3]$ & $2 / m_{z}[3]$ & $222[6]$ & $\mathrm{mm} 2_{x}[2]$ & $\mathrm{mm} 2_{y}[2]$ & $\mathrm{mm} 2_{z}[2]$ \\
\hline $22 \mathrm{P} 4$ & 8 & $2_{z}[5]$ & $4[3]$ & & & & & \\
\hline $23 \mathrm{I} 4$ & 7 & $2_{z}[5]$ & $4[2]$ & & & & & \\
\hline $24 \mathrm{P} \overline{4}$ & 10 & $2_{z}[5]$ & $\overline{4}[5]$ & & & & & \\
\hline $25 \mathrm{I} \overline{4}$ & 11 & $2{ }_{z}[5]$ & $\overline{4}[6]$ & & & & & \\
\hline $26 \mathrm{P} 4 / \mathrm{m}$ & 42 & $\overline{1}[8]$ & $2_{z}[5]$ & $\mathrm{m}_{z}[8]$ & $2 / \mathrm{m}_{z}[8]$ & $4[3]$ & $\overline{4}[5]$ & $4 / \mathrm{m}[5]$ \\
\hline $27 \mathrm{I} 4 / \mathrm{m}$ & 34 & $\overline{1}[4]$ & $2_{z}[5]$ & $\mathrm{m}_{z}[12]$ & $2 / \mathrm{m}_{z}[3]$ & $4[2]$ & $\overline{4}[6]$ & $4 / \mathrm{m}[2]$ \\
\hline $28 \mathrm{P} 422$ & 26 & $2_{z}[5]$ & $222[8]$ & $222_{d}[5]$ & $4[3]$ & $422[5]$ & & \\
\hline $29 \mathrm{I} 422$ & 18 & $2_{z}[5]$ & $222[6]$ & $222_{d}[3]$ & $4[2]$ & $422[2]$ & & \\
\hline $30 \mathrm{P} 4 \mathrm{~mm}$ & 19 & $2_{z}[5]$ & $\mathrm{mm} 2_{z}[5]$ & $\mathrm{mm} 2_{d}[3]$ & $4[3]$ & $4 \mathrm{~mm}[3]$ & & \\
\hline $31 \mathrm{I} 4 \mathrm{~mm}$ & 16 & $2_{z}[5]$ & $\mathrm{mm} 2_{z}[2]$ & $\mathrm{mm} 2_{d}[5]$ & $4[2]$ & $4 \mathrm{~mm}[2]$ & & \\
\hline $32 \mathrm{P} \overline{4} 2 \mathrm{~m}$ & 26 & $2_{z}[5]$ & $222[8]$ & $\mathrm{mm} 2_{d}[3]$ & $\overline{4}[5]$ & $\overline{4} 2 \mathrm{~m}[5]$ & & \\
\hline $33 \mathrm{P} \overline{4} \mathrm{~m} 2$ & 25 & $2_{z}[5]$ & $222_{d}[5]$ & $\mathrm{mm} 2_{z}[5]$ & $\overline{4}[5]$ & $\overline{4} \mathrm{~m} 2[5]$ & & \\
\hline $34 \mathrm{I} \overline{4} \mathrm{~m} 2$ & 18 & $2_{z}[5]$ & $222_{d}[3]$ & $\mathrm{mm} 2_{z}[2]$ & $\overline{4}[6]$ & $\overline{4} \mathrm{~m} 2[2]$ & & \\
\hline $35 \mathrm{I} \overline{4} 2 \mathrm{~m}$ & 28 & $2{ }_{z}[5]$ & $222[6]$ & $\mathrm{mm} 2_{d}[5]$ & $\overline{4}[6]$ & $\overline{4} 2 \mathrm{~m}[6]$ & & \\
\hline \multirow[t]{2}{*}{$36 \mathrm{P} 4 / \mathrm{mmm}$} & \multirow[t]{2}{*}{107} & $\overline{1}[8]$ & $2_{z}[5]$ & $\mathrm{m}_{z}[16]$ & $2 / m_{z}[8]$ & $222[8]$ & $222_{d}[5]$ & $\mathrm{mm} 2_{z}[5]$ \\
\hline & & $\mathrm{mm} 2_{d}[3]$ & $\mathrm{mmm}[8]$ & $\mathrm{mmm}_{d}[5]$ & $4[3]$ & $\overline{4}[5]$ & $4 / m[5]$ & $422[5]$ \\
\hline
\end{tabular}


Table 5.1 - continued from previous page

\begin{tabular}{|c|c|c|c|c|c|c|c|c|}
\hline \multirow{2}{*}{$\begin{array}{l}\mathrm{ACC} \\
36 \mathrm{P} 4 / \mathrm{mmm}\end{array}$} & \multirow{2}{*}{$\begin{array}{l}\text { total } \\
107\end{array}$} & \multicolumn{7}{|c|}{ cpg-normal subgroups and number of corresponding $B_{N}$ families } \\
\hline & & $4 \mathrm{~mm}[3]$ & $\overline{4} 2 \mathrm{~m}[5]$ & $\overline{4} \mathrm{~m} 2[5]$ & $4 / \mathrm{mmm}[5]$ & & & \\
\hline \multirow[t]{3}{*}{$37 \mathrm{I} 4 / \mathrm{mmm}$} & 69 & $\overline{1}[4]$ & $2_{z}[5]$ & $\mathrm{m}_{z}[12]$ & $2 / \mathrm{m}_{z}[3]$ & $222[6]$ & $222_{d}[3]$ & $\mathrm{mm} 2_{z}[2]$ \\
\hline & & $\mathrm{mm} 2_{d}[5]$ & $\mathrm{mmm}[2]$ & $\mathrm{mmm}_{d}[3]$ & $4[2]$ & $\overline{4}[6]$ & $4 / \mathrm{m}[2]$ & $422[2]$ \\
\hline & & $4 \mathrm{~mm}[2]$ & $\overline{4} 2 \mathrm{~m}[6]$ & $\overline{4} \mathrm{~m} 2[2]$ & $4 / \mathrm{mmm}[2]$ & & & \\
\hline $38 \mathrm{P} 3$ & 4 & $3[4]$ & & & & & & \\
\hline $39 \mathrm{R} 3$ & 1 & $3[1]$ & & & & & & \\
\hline $40 \mathrm{P} \overline{3}$ & 10 & $\overline{1}[4]$ & $3[4]$ & $\overline{3}[2]$ & & & & \\
\hline $41 \mathrm{R} \overline{3}$ & 7 & $\overline{1}[4]$ & $3[1]$ & $\overline{3}[2]$ & & & & \\
\hline 42 P312 & 6 & $3[4]$ & $312[2]$ & & & & & \\
\hline 43 P321 & 6 & $3[2]$ & $321[4]$ & & & & & \\
\hline 44 R32 & 3 & $3[1]$ & $321[2]$ & & & & & \\
\hline 45 P3m1 & 3 & $3[2]$ & $3 \mathrm{~m} 1[1]$ & & & & & \\
\hline 46 P31m & 8 & $3[4]$ & $31 \mathrm{~m}[4]$ & & & & & \\
\hline 47 R3m & 2 & $3[1]$ & $3 \mathrm{~m} 1[1]$ & & & & & \\
\hline 48 P $\overline{3} 1 \mathrm{~m}$ & 18 & $\overline{1}[4]$ & $3[4]$ & $\overline{3}[2]$ & $312[2]$ & $31 \mathrm{~m}[4]$ & $\overline{3} 1 \mathrm{~m}[2]$ & \\
\hline 49 P $3 \bar{m} 1$ & 15 & $\overline{1}[4]$ & $3[2]$ & $\overline{3}[2]$ & $321[4]$ & $3 \mathrm{~m} 1[1]$ & $\overline{3} \mathrm{~m} 1[2]$ & \\
\hline $50 \mathrm{R} \overline{3} \mathrm{~m}$ & 12 & $\overline{1}[4]$ & $3[1]$ & $\overline{3}[2]$ & $321[2]$ & $3 \mathrm{~m} 1[1]$ & $\overline{3} \mathrm{~m} 1[2]$ & \\
\hline 51 P6 & 5 & $2_{z}[2]$ & $3[2]$ & $6[1]$ & & & & \\
\hline $52 \mathrm{P} \overline{6}$ & 13 & $\mathrm{~m}_{z}[7]$ & $3[2]$ & $\overline{6}[4]$ & & & & \\
\hline \multirow[t]{2}{*}{53 P6/m } & 28 & $\overline{1}[4]$ & $2 z[2]$ & $\mathrm{m}_{z}[7]$ & $2 / \mathrm{m}_{z}[4]$ & $3[2]$ & $\overline{3}[2]$ & $6[1]$ \\
\hline & & $\overline{6}[4]$ & $6 / \mathrm{m}[2]$ & & & & & \\
\hline 54 P622 & 13 & $2_{z}[2]$ & $3[2]$ & $312[2]$ & $321[4]$ & $6[1]$ & $622[2]$ & \\
\hline 55 P6mm & 9 & $2_{z}[2]$ & $3[2]$ & $3 \mathrm{~m} 1[1]$ & $31 \mathrm{~m}[2]$ & $6[1]$ & $6 \mathrm{~mm}[1]$ & \\
\hline 56 Pб̄m2 & 16 & $\mathrm{~m}_{z}[5]$ & $3[2]$ & $312[2]$ & $3 \mathrm{~m} 1[1]$ & $\overline{6}[4]$ & $\overline{6} \mathrm{~m} 2[2]$ & \\
\hline $57 \mathrm{P} \overline{6} 2 \mathrm{~m}$ & 21 & $\mathrm{~m}_{z}[5]$ & $3[2]$ & $321[4]$ & $31 \mathrm{~m}[2]$ & $\overline{6}[4]$ & $\overline{6} 2 \mathrm{~m}[4]$ & \\
\hline \multirow[t]{3}{*}{$58 \mathrm{P} 6 / \mathrm{mmm}$} & 50 & $\overline{1}[4]$ & $2 z[2]$ & $\mathrm{m}_{z}[5]$ & $2 / \mathrm{m}_{z}[4]$ & $3[2]$ & $\overline{3}[2]$ & $312[2]$ \\
\hline & & $321[4]$ & $3 \mathrm{~m} 1[1]$ & $31 \mathrm{~m}[2]$ & $\overline{3} 1 \mathrm{~m}[2]$ & $\overline{3} \mathrm{~m} 1[2]$ & $6[1]$ & $\overline{6}[4]$ \\
\hline & & $6 / \mathrm{m}[2]$ & $622[2]$ & $6 \mathrm{~mm}[1]$ & $\overline{6} \mathrm{~m} 2[2]$ & $\overline{6} 2 \mathrm{~m}[4]$ & $6 / \mathrm{mmm}[2]$ & \\
\hline
\end{tabular}


Table 5.1 - continued from previous page

\begin{tabular}{|c|c|c|c|c|c|c|c|c|}
\hline ACC & total & \multicolumn{7}{|c|}{ cpg-normal subgroups and number of corresponding $B_{N}$ families } \\
\hline $59 \mathrm{P} 23$ & 6 & $222[4]$ & $23[2]$ & & & & & \\
\hline $60 \mathrm{~F} 23$ & 3 & $222[2]$ & $23[1]$ & & & & & \\
\hline $61 \mathrm{I} 23$ & 12 & $222[6]$ & $23[6]$ & & & & & \\
\hline $62 \mathrm{Pm} \overline{3}$ & 16 & $\overline{1}[4]$ & $222[4]$ & $\mathrm{mmm}[4]$ & $23[2]$ & $\mathrm{m} \overline{3}[2]$ & & \\
\hline $63 \mathrm{Fm} \overline{3}$ & 9 & $\overline{1}[3]$ & $222[2]$ & $\mathrm{mmm}[2]$ & $23[1]$ & $\mathrm{m} \overline{3}[1]$ & & \\
\hline $64 \operatorname{Im} \overline{3}$ & 19 & $\overline{1}[3]$ & $222[6]$ & $\mathrm{mmm}[2]$ & $23[6]$ & $\mathrm{m} \overline{3}[2]$ & & \\
\hline 65 P432 & 8 & $222[4]$ & $23[2]$ & $432[2]$ & & & & \\
\hline 66 F432 & 4 & $222[2]$ & $23[1]$ & $432[1]$ & & & & \\
\hline 67 I432 & 14 & $222[6]$ & $23[6]$ & $432[2]$ & & & & \\
\hline $68 \mathrm{P} \overline{4} 3 \mathrm{~m}$ & 8 & $222[4]$ & $23[2]$ & $\overline{4} 3 \mathrm{~m}[2]$ & & & & \\
\hline $69 \mathrm{~F} \overline{4} 3 \mathrm{~m}$ & 4 & $222[2]$ & $23[1]$ & $\overline{4} 3 \mathrm{~m}[1]$ & & & & \\
\hline $70 \mathrm{I} \overline{4} 3 \mathrm{~m}$ & 18 & $222[6]$ & $23[6]$ & $\overline{4} 3 \mathrm{~m}[6]$ & & & & \\
\hline $71 \mathrm{Pm} \overline{3} \mathrm{~m}$ & 22 & $\begin{array}{l}\overline{1}[4] \\
\mathrm{m} \overline{3} \mathrm{~m}[2]\end{array}$ & $222[4]$ & $\operatorname{mmm}[4]$ & $23[2]$ & $\mathrm{m} \overline{3}[2]$ & $432[2]$ & $\overline{4} 3 \mathrm{~m}[2]$ \\
\hline $72 \mathrm{Fm} \overline{3} \mathrm{~m}$ & 12 & $\begin{array}{l}\overline{1}[3] \\
\mathrm{m} \overline{3} \mathrm{~m}[1]\end{array}$ & $222[2]$ & $\mathrm{mmm}[2]$ & $23[1]$ & $\mathrm{m} \overline{3}[1]$ & $432[1]$ & $\overline{4} 3 \mathrm{~m}[1]$ \\
\hline $73 \operatorname{Im} \overline{3} \mathrm{~m}$ & 29 & $\begin{array}{l}\overline{1}[3] \\
\mathrm{m} \overline{3} \mathrm{~m}[2]\end{array}$ & $222[6]$ & $\mathrm{mmm}[2]$ & $23[6]$ & $\mathrm{m} \overline{3}[2]$ & $432[2]$ & $\overline{4} 3 \mathrm{~m}[6]$ \\
\hline
\end{tabular}

Consider the arithmetic class represented by crystallographic space group Pmmm (\#18 in Table 5.1) which has $m_{x}, m_{y}$, and $m_{z}$ each listed as separate cpg-normal subgroups, where $m_{x}$ and $m_{y}$ both have $11 B_{N}$ families while $m_{z}$ only has 8 . Even the $B_{N}$ families of $m_{x}$ and $m_{y}$ look very different; no numerical instance of one is contained in the other. Because $m_{x}, m_{y}$, and $m_{z}$ are equivalent in some sense (they can be conjugate subgroups of the normalizer of Pmmm with respect to Euclidean transformations), this is immediately surprising. The resolution of this paradox lies in the fact that $B_{N}$ is presented as a column-HNF matrix, which is lower triangular so that the $x, y$ and $z$ basis vectors can have different numbers of non-zero values. Thus, while the $B_{N}$ families of 
these cpg-normal subgroups look very different, one can swap the first two rows of any numeric instance of $B_{N}$ associated with $m_{x}$ and reduce the result again to a column-HNF matrix to obtain the corresponding numerical instance of $B_{N}$ associated with $m_{y}$.

Of the four cpg-normal subgroups of ACC Amm2 (\#15), cpg-normal subgroups $2{ }_{z}, m_{y}$, and $m m 2 z$ each have 3 or fewer $B_{N}$ families, while $m_{x}$ has an impressive $77 B_{N}$ families. This latter cpg-normal subgroup proved exceptional in a variety of ways. Of the list of 74 unique conditions listed in Table A.2, this case alone contributes 24 of them, including the only 4 cases where negative variable values are allowed. ACCs that stood out amongst the others in terms of complexity were those based on point groups $m m m, 4 / m m m$, and $6 / \mathrm{mmm}$. Here, the large numbers of total $B_{N}$ families per ACC arise due to large numbers of cpg-normal subgroups, and not because any one normal subgroup provides an extraordinary number of $B_{N}$ families.

The $B_{N}$ matrices of Table A.1 are all presented in the primitive HNF setting of the parent space group. To transform a $B_{N}$ matrix from this table into the more familiar conventional setting, we multiply it on the left by the appropriate transformation matrix from the list below according to lattice-centering type (e.g. P, A, C, I, F, or R).

$$
\begin{gathered}
Q(P)=\left(\begin{array}{ccc}
1 & 0 & 0 \\
0 & 1 & 0 \\
0 & 0 & 1
\end{array}\right) \quad Q(A)=\left(\begin{array}{ccc}
1 & 0 & 0 \\
0 & \frac{1}{2} & -\frac{1}{2} \\
0 & \frac{1}{2} & \frac{1}{2}
\end{array}\right) \quad Q(C)=\left(\begin{array}{ccc}
\frac{1}{2} & -\frac{1}{2} & 0 \\
\frac{1}{2} & \frac{1}{2} & 0 \\
0 & 0 & 1
\end{array}\right) \\
Q(F)=\left(\begin{array}{lll}
0 & \frac{1}{2} & \frac{1}{2} \\
\frac{1}{2} & 0 & \frac{1}{2} \\
\frac{1}{2} & \frac{1}{2} & 0
\end{array}\right) \quad Q(I)=\left(\begin{array}{ccc}
-\frac{1}{2} & \frac{1}{2} & \frac{1}{2} \\
\frac{1}{2} & -\frac{1}{2} & \frac{1}{2} \\
\frac{1}{2} & \frac{1}{2} & -\frac{1}{2}
\end{array}\right) \quad Q(R)=\left(\begin{array}{ccc}
\frac{2}{3} & -\frac{1}{3} & -\frac{1}{3} \\
\frac{1}{3} & \frac{1}{3} & -\frac{2}{3} \\
\frac{1}{3} & \frac{1}{3} & \frac{1}{3}
\end{array}\right)
\end{gathered}
$$

As an example, if the sublattice basis in the primitive HNF setting of a face-centered cubic 
parent lattice is

$$
B_{N}=\left(\begin{array}{ccc}
1 & 0 & 0 \\
1 & 2 & 0 \\
1+c_{1} & c_{1} & 2+c_{1}
\end{array}\right)
$$

the new basis in the conventional face-centered setting would be

$$
Q(F) B_{N}=\left(\begin{array}{ccc}
0 & \frac{1}{2} & \frac{1}{2} \\
\frac{1}{2} & 0 & \frac{1}{2} \\
\frac{1}{2} & \frac{1}{2} & 0
\end{array}\right)\left(\begin{array}{ccc}
1 & 0 & 0 \\
1 & 2 & 0 \\
1+c_{1} & c_{1} & 2+c_{1}
\end{array}\right)=\left(\begin{array}{ccc}
\frac{2+c_{1}}{2} & \frac{2+c_{1}}{2} & \frac{2+c_{1}}{2} \\
\frac{2+c_{1}}{2} & \frac{c_{1}}{2} & \frac{2+c_{1}}{2} \\
1 & 1 & 0
\end{array}\right) .
$$

Obviously, this is no longer in Hermite normal form.

\subsection{Validation}

Extensive brute-force testing of Table A.1 helped us to identify and resolve problems with the computer algorithm. Though we have considerable confidence in the present table, we emphasize that it is only practical to run tests over a finite range of the infinite space of possible numerical $B_{N}$ matrices. It is possible that a wider search would uncover problems that we have missed. For this reason, we will make our code publicly available. To verify the legitimacy of our output, we have performed the following three tests.

For the first test, a matrix is compared to the list of $B_{N}$ matrices that have been tabulated. This matrix is also checked to see if it supports normality by checking if it satisfies equations 3.9 and 3.11. The matrix should fit one of our tabulated $B_{N}$ forms if and only if it supports normality. If we find a matrix that fits one of our $B_{N}$ forms but does not support normality or does not fit any form but does support normality then we know that there is an error in our tabulations. We have used this test with every possible Hermite normal form matrix with entries of values equal to or less than 6. 
The second test focused on the variables in the $B_{N}$ matrices. For each of our tabulated $B_{N}$, if there are no variables we simply check that it supports normality. If there are variables, we plug in values for those variables to make a matrix. The values that are plugged in are checked to see if they satisfy any extra conditions that the $B_{N}$ has (if any) and the matrix is then checked to see if it supports normality. If there are any values that satisfy one but not the other then there is an error in our tabulations. We have applied this test plugging in every combination of values up to 50 for every one of our tabulated $B_{N}$ matrices.

The third test focused on extra conditions and was not applied to any $B_{N}$ that had no extra conditions. For each case, different values are plugged in for each variable in a systematic order until a combination of values that satisfy all extra conditions in a triplet are found. Then these values are plugged into the $B_{N}$ matrix and it is checked that is supports normality. We have used this test to find every combination of values up to 250 that satisfy each set of extra conditions and verify that each solution satisfies normality. The results of this test were also used as a brute force method to check if there were even any solutions for each set of extra conditions (there were some cases that had no solutions or had a finite number of solutions). 


\section{Chapter 6}

\section{Examples}

\subsection{A Simple Case}

Consider space group $P 312$ (\#149), the point group of which has six elements: $P_{G}=\{(x, y, z)$, $(y,-x-y, z),(-x-y, x, z),(-y,-x,-z),(x+y,-y,-z),(-x, x+y,-z)\} . P_{G}$ has three normal subgroups: $\{(x, y, z),(y,-x-y, z),(-x-y, x, z),(-y,-x,-z),(x+y,-y,-z),(-x, x+y,-z)\}$, $\{(x, y, z),(y,-x-y, z),(-x-y, x, z)\}$, and $\{(x, y, z)\}$. For this example, let us consider the normal subgroup $P_{N}=\{(x, y, z),(y,-x-y, z),(-x-y, x, z)\}$. The lattice-coordinate matrix representation of the elements of $P_{G}$ is

$$
\begin{gathered}
\left\{\left(\begin{array}{lll}
1 & 0 & 0 \\
0 & 1 & 0 \\
0 & 0 & 1
\end{array}\right),\left(\begin{array}{ccc}
0 & -1 & 0 \\
1 & -1 & 0 \\
0 & 0 & 1
\end{array}\right),\left(\begin{array}{ccc}
-1 & 1 & 0 \\
-1 & 0 & 0 \\
0 & 0 & 1
\end{array}\right),\right. \\
\left.\left(\begin{array}{ccc}
0 & -1 & 0 \\
-1 & 0 & 0 \\
0 & 0 & -1
\end{array}\right),\left(\begin{array}{ccc}
1 & 0 & 0 \\
1 & -1 & 0 \\
0 & 0 & -1
\end{array}\right),\left(\begin{array}{ccc}
-1 & 1 & 0 \\
0 & 1 & 0 \\
0 & 0 & -1
\end{array}\right)\right\}
\end{gathered}
$$


so that $P_{N}$ is represented as

$$
\left\{\left(\begin{array}{lll}
1 & 0 & 0 \\
0 & 1 & 0 \\
0 & 0 & 1
\end{array}\right),\left(\begin{array}{ccc}
0 & -1 & 0 \\
1 & -1 & 0 \\
0 & 0 & 1
\end{array}\right),\left(\begin{array}{ccc}
-1 & 1 & 0 \\
-1 & 0 & 0 \\
0 & 0 & 1
\end{array}\right)\right\}
$$

When we impose equation 3.9 on each of the point operators of $P_{N}$, the identity yields only trivial conditions, whereas the other two elements yield

$$
B_{N}^{-1}\left(\begin{array}{ccc}
1 & 1 & 0 \\
-1 & 2 & 0 \\
0 & 0 & 0
\end{array}\right)=\left(\begin{array}{ccc}
\frac{1}{B_{11}} & \frac{1}{B_{11}} & 0 \\
-\frac{1}{B_{22}}-\frac{B_{21}}{B_{11} B_{22}} & \frac{2}{B_{22}}-\frac{B_{21}}{B_{11} B_{22}} & 0 \\
\frac{B_{32}}{B_{22} B_{33}}+\frac{-B_{22} B_{31}+B_{21} B_{32}}{B_{11} B_{22} B_{33}} & \frac{-2 B_{32}}{B_{22} B_{33}}+\frac{-B_{22} B_{31}+B_{21} B_{32}}{B_{11} B_{22} B_{33}} & 0
\end{array}\right) \in \mathbb{Z}^{3 \times 3}
$$

and

$$
B_{N}^{-1}\left(\begin{array}{ccc}
2 & -1 & 0 \\
1 & 1 & 0 \\
0 & 0 & 0
\end{array}\right)=\left(\begin{array}{ccc}
\frac{2}{B_{11}} & -\frac{1}{B_{11}} & 0 \\
\frac{1}{B_{22}}-\frac{2 B_{21}}{B_{11} B_{22}} & \frac{1}{B_{22}}+\frac{B_{21}}{B_{11} B_{22}} & 0 \\
\frac{-B_{32}}{B_{22} B_{33}}+\frac{-2 B_{22} B_{31}+2 B_{21} B_{32}}{B_{11} B_{22} B_{33}} & -\frac{B_{32}}{B_{22} B_{33}}-\frac{-B_{22} B_{31}+B_{21} B_{32}}{B_{11} B_{22} B_{33}} & 0
\end{array}\right) \in \mathbb{Z}^{3 \times 3}
$$

By inspection, it can be seen that equation 6.3 requires that $\frac{1}{B_{11}} \in \mathbb{Z}$. Because $B_{N}$ is in Hermite normal form, this is only possible if $B_{11}=1$. Rather than manually applying such logic successively to each element of $B_{N}$, we simply set $B_{11}=1$ and then used Mathematica's Reduce function to simultaneously require that every element be integral. The result is that $B_{N}$ must take one of the following forms:

$$
\begin{gathered}
B_{N}=\left(\begin{array}{ccc}
1 & 0 & 0 \\
0 & 1 & 0 \\
0 & 0 & c_{1}+1
\end{array}\right),\left(\begin{array}{ccc}
1 & 0 & 0 \\
2 & 3 & 0 \\
0 & 0 & c_{1}+1
\end{array}\right), \\
\left(\begin{array}{ccc}
1 & 0 & 0 \\
0 & 1 & 0 \\
2 c_{1}+2 & 2 c_{1}+2 & 3 c_{1}+3
\end{array}\right),\left(\begin{array}{ccc}
1 & 0 & 0 \\
0 & 1 & 0 \\
c_{1}+1 & c_{1}+1 & 3 c_{1}+3
\end{array}\right)
\end{gathered}
$$


where $c_{1}$ can be any non-negative integer. Now let us try to further restrict the four $B_{N}$ families in equation 6.5 by imposing equation 3.11 on each one separately.

For the first matrix, we must have

$$
\left(\begin{array}{ccc}
1 & 0 & 0 \\
0 & 1 & 0 \\
0 & 0 & \frac{1}{c_{1}+1}
\end{array}\right) p_{g}\left(\begin{array}{ccc}
1 & 0 & 0 \\
0 & 1 & 0 \\
0 & 0 & c_{1}+1
\end{array}\right) \in \mathbb{Z}^{3 \times 3}
$$

for every $p_{g} \in P_{G}$. However, by inspection, we can see that every $p_{g}$ commutes with the matrix so that the condition reduces to $p_{g} \in \mathbb{Z}^{3 \times 3}$, which does not further restrict $B_{N}$.

For the second matrix, the application of equation 3.11 to the five non-identity elements of $P_{G}$ yields the following matrices:

$$
\left\{\left(\begin{array}{ccc}
-2 & -3 & 0 \\
1 & 1 & 0 \\
0 & 0 & 1
\end{array}\right),\left(\begin{array}{ccc}
1 & 3 & 0 \\
-1 & -2 & 0 \\
0 & 0 & 1
\end{array}\right),\left(\begin{array}{ccc}
-2 & -3 & 0 \\
1 & 2 & 0 \\
0 & 0 & -1
\end{array}\right),\left(\begin{array}{ccc}
1 & 0 & 0 \\
-1 & -1 & 0 \\
0 & 0 & -1
\end{array}\right),\left(\begin{array}{ccc}
1 & 3 & 0 \\
0 & -1 & 0 \\
0 & 0 & -1
\end{array}\right)\right\},
$$

none of which contain fractions or depend on $c_{1}$ and thus do not further restrict $B_{N}$.

Application of equation 3.11 to the third and fourth matrices yields

$$
\begin{gathered}
\left\{\left(\begin{array}{ccc}
0 & -1 & 0 \\
1 & -1 & 0 \\
0 & 2 & 1
\end{array}\right),\left(\begin{array}{ccc}
-1 & 1 & 0 \\
-1 & 0 & 0 \\
2 & 0 & 1
\end{array}\right),\left(\begin{array}{ccc}
0 & -1 & 0 \\
-1 & 0 & 0 \\
0 & 0 & -1
\end{array}\right),\right. \\
\left.\left(\begin{array}{ccc}
1 & 0 & 0 \\
1 & -1 & 0 \\
-2 & 0 & -1
\end{array}\right),\left(\begin{array}{ccc}
-1 & 1 & 0 \\
0 & 1 & 0 \\
0 & -2 & -1
\end{array}\right)\right\} \in \mathbb{Z}^{3 \times 3}
\end{gathered}
$$


and

$$
\begin{gathered}
\left\{\left(\begin{array}{ccc}
0 & -1 & 0 \\
1 & -1 & 0 \\
0 & 1 & 1
\end{array}\right),\left(\begin{array}{ccc}
-1 & 1 & 0 \\
-1 & 0 & 0 \\
1 & 0 & 1
\end{array}\right),\left(\begin{array}{ccc}
0 & -1 & 0 \\
-1 & 0 & 0 \\
0 & 0 & -1
\end{array}\right),\right. \\
\left.\left(\begin{array}{ccc}
1 & 0 & 0 \\
1 & -1 & 0 \\
-1 & 0 & -1
\end{array}\right),\left(\begin{array}{ccc}
-1 & 1 & 0 \\
0 & 1 & 0 \\
0 & -1 & -1
\end{array}\right)\right\} \in \mathbb{Z}^{3 \times 3}
\end{gathered}
$$

respectively. Again, both of these conditions are clearly true without further restricting the form of $B_{N}$.

For this particular $G$ and $P_{N}$, imposing equation 3.11 does not restrict our solution any further. Hence, the four infinite families in equation 6.5 span all the possible normally supportive sublattices for the space group $P 312$ (\#149) and this particular normal cpg-subgroup $P_{N}$.

\subsection{A Case with Bifurcation}

Consider space group Pmm2 (\#25), which has a point group $P_{G}=\{(x, y, z),(-x,-y, z)$, $(-x, y, z),(x,-y, z)\}$ with the lattice coordinate representation

$$
\left\{\left(\begin{array}{lll}
1 & 0 & 0 \\
0 & 1 & 0 \\
0 & 0 & 1
\end{array}\right),\left(\begin{array}{ccc}
-1 & 0 & 0 \\
0 & -1 & 0 \\
0 & 0 & 1
\end{array}\right),\left(\begin{array}{ccc}
-1 & 0 & 0 \\
0 & 1 & 0 \\
0 & 0 & 1
\end{array}\right),\left(\begin{array}{ccc}
1 & 0 & 0 \\
0 & -1 & 0 \\
0 & 0 & 1
\end{array}\right)\right\} .
$$

$P_{G}$ has the following five normal subgroups: $\{(x, y, z)\},\{(x, y, z),(-x,-y, z)\},\{(x, y, z),(-x, y, z)\}$, $\{(x, y, z),(x,-y, z)\}$, and $\{(x, y, z),(-x,-y, z),(-x, y, z),(x,-y, z)\}$. Let us consider the case of $P_{N}=m_{y}=\{(x, y, z),(x,-y, z)\}$.

When we impose equation 3.9 on the non-identity elements of $P_{N}$, Mathematica's Reduce 
function yields the following eight $B_{N}$ families:

$$
\begin{gathered}
B_{N}=\left(\begin{array}{ccc}
1+c_{1} & 0 & 0 \\
0 & 1 & 0 \\
2 c_{2} & 1+c_{2}+c_{3} & 2+2 c_{2}+2 c_{3}
\end{array}\right),\left(\begin{array}{ccc}
1+c_{1} & 0 & 0 \\
0 & 1 & 0 \\
1+2 c_{2} & 1+c_{2}+c_{3} & 2+2 c_{2}+2 c_{3}
\end{array}\right) \\
\left(\begin{array}{ccc}
1+c_{1} & 0 & 0 \\
0 & 1 & 0 \\
2 c_{2} & 0 & 2+2 c_{2}+2 c_{3}
\end{array}\right),\left(\begin{array}{ccc}
1+c_{1} & 0 & 0 \\
0 & 1 & 0 \\
2 c_{2} & 0 & 1+2 c_{2}+2 c_{3}
\end{array}\right),\left(\begin{array}{ccc}
1+c_{1} & 0 & 0 \\
0 & 1 & 0 \\
1+c_{1} & 0 & 0 \\
0 & 1 & 0 \\
1+2 c_{2} & 0 & 3+2 c_{2}+2 c_{3}
\end{array}\right),\left(\begin{array}{ccc}
1+c_{1} & 0 & 2+2 c_{2}+2 c_{3}
\end{array}\right) \\
0 \\
\left(\begin{array}{ccc}
1+c_{2} & 0 & 0 \\
c_{2} & 0 & 1+c_{2}+c_{3}
\end{array}\right)
\end{gathered}
$$

where $c_{1}, c_{2}$, and $c_{3}$ can be any non-negative integers.

For the sake of brevity, we'll only consider the first $B_{N}$ family in equation 6.11 , to which the application of equation 3.11 yields the condition

$$
\left(\begin{array}{ccc}
\frac{1}{1+c_{1}} & 0 & 0 \\
0 & 1 & 0 \\
-\frac{c_{2}}{\left(1+c_{1}\right)\left(1+c_{2}+c_{3}\right)} & -\frac{1}{2} & \frac{1}{2+2 c_{2}+2 c_{3}}
\end{array}\right) \operatorname{pg}\left(\begin{array}{ccc}
1+c_{1} & 0 & 0 \\
0 & 1 & 0 \\
2 c_{2} & 1+c_{2}+c_{3} & 2+2 c_{2}+2 c_{3}
\end{array}\right) \in \mathbb{Z}^{3 \times 3}
$$

for each $p_{g} \in P_{G}$. Inserting each $p_{g}$ then yields

$$
\left\{\left(\begin{array}{lll}
1 & 0 & 0 \\
0 & 1 & 0 \\
0 & 0 & 1
\end{array}\right),\left(\begin{array}{ccc}
-1 & 0 & 0 \\
0 & -1 & 0 \\
\frac{2 c_{2}}{1+c_{2}+c_{3}} & 1 & 1
\end{array}\right),\left(\begin{array}{ccc}
-1 & 0 & 0 \\
0 & 1 & 0 \\
\frac{2 c_{2}}{1+c_{2}+c_{3}} & 0 & 1
\end{array}\right),\left(\begin{array}{ccc}
1 & 0 & 0 \\
0 & -1 & 0 \\
0 & 1 & 1
\end{array}\right)\right\} \in \mathbb{Z}^{3 \times 3}
$$

which has only one nontrivial condition: $\frac{2 c_{2}}{1+c_{2}+c_{3}} \in \mathbb{Z}$. There are two ways to satisfy this condition. The first is to let $c_{2}=0$ and the second is to let $c_{2}=c_{3}+1$. Applying these two replacements 
separately effectively bifurcates the original $B_{N}$ family into the following two families:

$$
\left(\begin{array}{ccc}
1+c_{1} & 0 & 0 \\
0 & 1 & 0 \\
0 & 1+c_{2} & 2+2 c_{2}
\end{array}\right) \text { and }\left(\begin{array}{ccc}
1+c_{1} & 0 & 0 \\
0 & 1 & 0 \\
2+2 c_{2} & 2+2 c_{2} & 4+4 c_{2}
\end{array}\right)
$$

where we have relabeled the old $c_{3}$ as the new $c_{2}$ for convenience, and where $c_{1}$ and $c_{2}$ can be any non-negative integers.

\subsection{A Case with Extra Conditions}

Consider space group Imm2 (\#44) which has a point group $P_{G}=m m 2_{z}$ whose point-group operators in the primitive setting are $\{(x, y, z),(y-z, x-z,-z),(x, x-z, x-y),(y-z, y,-x+y)\}$ with the lattice coordinate representation

$$
\left\{\left(\begin{array}{lll}
1 & 0 & 0 \\
0 & 1 & 0 \\
0 & 0 & 1
\end{array}\right),\left(\begin{array}{ccc}
0 & 1 & 0 \\
1 & 0 & 0 \\
-1 & -1 & -1
\end{array}\right),\left(\begin{array}{ccc}
1 & 1 & 1 \\
0 & 0 & -1 \\
0 & -1 & 0
\end{array}\right),\left(\begin{array}{ccc}
0 & 0 & -1 \\
1 & 1 & 1 \\
-1 & 0 & 0
\end{array}\right)\right\} .
$$

$P_{G}$ has the following five normal subgroups: $\{(x, y, z)\},\{(x, y, z),(y-z, x-z,-z)\},\{(x, y, z),(x, x-$ $z, x-y)\},\{(x, y, z),(y-z, y,-x+y)\}$, and $\{(x, y, z),(y-z, x-z,-z),(x, x-z, x-y),(y-z, y,-x+$ $y)\}$. Let us consider the case of $P_{N}=m_{x}=\{(x, y, z),(x, x-z, x-y)\}$.

When we impose equation 3.9 on the non-identity elements of $P_{N}$, Mathematica's Reduce function yields the following three $B_{N}$ families:

$$
B_{N}=\left(\begin{array}{ccc}
1+c_{1} & 0 & 0 \\
0 & 1 & 0 \\
0 & 0 & 1
\end{array}\right),\left(\begin{array}{ccc}
1+c_{1} & 0 & 0 \\
0 & 1 & 0 \\
c_{2} & 1 & 2+c_{2}+c_{3}
\end{array}\right),\left(\begin{array}{ccc}
1+c_{1} & 0 & 0 \\
0 & 1 & 0 \\
1+c_{2} & 1 & 2+c_{2}+c_{3}
\end{array}\right)
$$

where $c_{1}, c_{2}$, and $c_{3}$ can be any non-negative integers. 
For this example, we'll only consider the third $B_{N}$ family in equation 6.16 , to which the application of equation 3.11 yields the condition

$$
\left(\begin{array}{ccc}
\frac{1}{1+c_{1}} & 0 & 0 \\
0 & 1 & 0 \\
-\frac{1+c_{2}}{\left(1+c_{1}\right)\left(2+c_{2}+c_{3}\right)} & -\frac{1}{2+c_{2}+c_{3}} & \frac{1}{2+c_{2}+c_{3}}
\end{array}\right) \operatorname{pg}\left(\begin{array}{ccc}
1+c_{1} & 0 & 0 \\
0 & 1 & 0 \\
1+c_{2} & 1 & 2+c_{2}+c_{3}
\end{array}\right) \in \mathbb{Z}^{3 \times 3}
$$

for each $p_{g} \in P_{G}$. Inserting each $p_{g}$ then yields

$$
\begin{gathered}
\left(\begin{array}{lll}
1 & 0 & 0 \\
0 & 1 & 0 \\
0 & 0 & 1
\end{array}\right),\left(\begin{array}{ccc}
-\frac{1+c_{2}}{1+c_{1}} & 0 & -\frac{2+c_{2}+c_{3}}{1+c_{1}} \\
c_{1}-c_{2} & -1 & -2-c_{2}-c_{3} \\
-\frac{\left(c_{1}-c_{2}\right)\left(2+c_{1}+c_{2}\right)}{\left(1+c_{1}\right)\left(2+c_{2}+c_{3}\right)} & 0 & \frac{1+c_{2}}{1+c_{1}}
\end{array}\right), \\
\left(\begin{array}{ccccc}
1 & 0 & 0 \\
c_{1}-c_{2} & -1 & -2-c_{2}-c_{3} \\
0 & 0 & 1
\end{array}\right),\left(\begin{array}{ccc}
1+c_{1} & 0 & -\frac{2+c_{2}+c_{3}}{1+c_{1}} \\
0 & 1 & 0 \\
-\frac{\left(c_{1}-c_{2}\right)\left(2+c_{1}+c_{2}\right)}{\left(1+c_{1}\right)\left(2+c_{2}+c_{3}\right)} & 0 & \frac{1+c_{2}}{1+c_{1}}
\end{array}\right) \in \mathbb{Z}^{3 \times 3} .
\end{gathered}
$$

This essentially has three nontrivial conditions: $\left\{d_{1}=\frac{1+c_{2}}{1+c_{1}}, d_{2}=\frac{2+c_{2}+c_{3}}{1+c_{1}}, d_{3}=\frac{\left(c_{1}-c_{2}\right)\left(2+c_{1}+c_{2}\right)}{\left(1+c_{1}\right)\left(2+c_{2}+c_{3}\right)}\right\}$ $\in \mathbb{Z}$. Using Mathematica's Reduce command under the assumption that all $c$ and $d$ variables are integers, we find that we can satisfy both of these conditions by bifurcating this $B_{N}$ family into the following three cases.

For the first case, we let $d_{1}=1$ and $d_{2}=2$ by setting $c_{2}=c_{1}$ and $c_{3}=-2-c_{1}+2+2 c_{1}$. Making these substitutions will change $d_{3}$ to 0 , which is trivially integral, and simplify $B_{N}$ to

$$
\left(\begin{array}{ccc}
1+c_{1} & 0 & 0 \\
0 & 1 & 0 \\
1+c_{1} & 1 & 2+2 c_{1}
\end{array}\right)
$$

where $c_{1}$ can be any non-negative integer.

For the second case, we let $d_{1}=1$ by setting $c_{2}=c_{1}$ and $c_{3}=-2-c_{1}+d_{2}+c_{1} d_{2}$. Making 
these substitutions reduces $d_{3}$ to 0 , which is trivially integral, and changes $B_{N}$ to

$$
\left(\begin{array}{ccc}
1+c_{1} & 0 & 0 \\
0 & 1 & 0 \\
1+c_{1} & 1 & d_{2}+c_{1} d_{2}
\end{array}\right)
$$

Because $d_{2}=\frac{2+c_{1}+c_{3}}{1+c_{1}}$, where $c_{1}$ and $c_{3}$ can take on any non-negative integers, an appropriate value of $c_{3}$ will achieve any desired integer value of $d_{2}$ greater than 1 for any given non-negative integer value of $c_{1}$. Thus, the range of $d_{2}$ is $d_{2} \geq 2$. However, because we have already treated the $d_{2}=2$ case above, we will restrict the new $B_{N}$ to the range $d_{2} \geq 3$ in order to ensure that all $B_{N}$ families are mutually disjoint. Finally, because it is convenient to define each variable in the $B_{N}$ family over the range of non-negative integers, because $d_{2}$ does not appear in any extra conditions, and because the old $c_{2}$ variable is no longer being used, we define a new variable $c_{2}=d_{2}-3 \geq 0$ and replace $d_{2}$ with $c_{2}+3$ within $B_{N}$ to obtain

$$
\left(\begin{array}{ccc}
1+c_{1} & 0 & 0 \\
0 & 1 & 0 \\
1+c_{1} & 1 & \left(1+c_{1}\right)\left(3+c_{2}\right)
\end{array}\right)
$$

where $c_{1}$ and $c_{2}$ can be any non-negative integers.

For the third case, we let $c_{2}=-1+d_{1}+c_{1} d_{1}$ and $c_{3}=-1-d_{1}-c_{1} d_{1}+d_{2}+c_{1} d_{2}$, which reduces the $d_{3}$ condition to $\frac{1-d_{1}^{2}}{d_{2}} \in \mathbb{Z}$ and $B_{N}$ to

$$
\left(\begin{array}{ccc}
1+c_{1} & 0 & 0 \\
0 & 1 & 0 \\
d_{1}+c_{1} d_{1} & 1 & d_{2}+c_{1} d_{2}
\end{array}\right)
$$

Using similar logic to that applied in the previous case, and skipping the previously-treated $d_{1}=1$ scenario, our $d$-variable ranges are now $d_{1} \geq 2$ and $d_{2} \geq 1+d_{1}$. For convenience, we replace $d_{1}$ with $2+d_{1}$ and $d_{2}$ with $3+d_{1}+d_{2}$. Making these substitutions reduces the $d_{3}$ condition to 
$\frac{3+4 d_{1}+d_{1}^{2}}{3+d_{1}+d_{2}} \in \mathbb{Z}$ and $B_{N}$ to

$$
\left(\begin{array}{ccc}
1+c_{1} & 0 & 0 \\
0 & 1 & 0 \\
\left(1+c_{1}\right)\left(2+d_{1}\right) & 1 & \left(1+c_{1}\right)\left(3+d_{1}+d_{2}\right)
\end{array}\right)
$$

where $c_{1}, d_{1}$, and $d_{2}$ can be any non-negative integers that satisfy $\frac{3+4 d_{1}+d_{1}^{2}}{3+d_{1}+d_{2}} \in \mathbb{Z}$.

\subsection{A Very Messy Case}

Consider space group Amm2 (\#38) which has a point group $P_{G}=m m 2_{z}$ whose point-group operators in the primitive setting are $\{(x, y, z),(-x, z, y),(-x, y, z),(x, z, y)\}$ with the lattice coordinate representation

$$
\left\{\left(\begin{array}{lll}
1 & 0 & 0 \\
0 & 1 & 0 \\
0 & 0 & 1
\end{array}\right),\left(\begin{array}{ccc}
-1 & 0 & 0 \\
0 & 0 & 1 \\
0 & 1 & 0
\end{array}\right),\left(\begin{array}{ccc}
-1 & 0 & 0 \\
0 & 1 & 0 \\
0 & 0 & 1
\end{array}\right),\left(\begin{array}{lll}
1 & 0 & 0 \\
0 & 0 & 1 \\
0 & 1 & 0
\end{array}\right)\right\} .
$$

$P_{G}$ has the following five normal subgroups: $\{(x, y, z)\},\{(x, y, z),(-x, z, y)\},\{(x, y, z),(-x, y, z)\}$, $\{(x, y, z),(x, z, y)\}$, and $\{(x, y, z),(-x, z, y),(-x, y, z),(x, z, y)\}$. Let us consider the case of $P_{N}=$ $m_{x}=\{(x, y, z),(-x, y, z)\}$.

When we impose equation 3.9 on the non-identity elements of $P_{N}$, Mathematica's Reduce 
function yields the following eight $B_{N}$ families:

$$
\begin{aligned}
& B_{N}=\left(\begin{array}{ccc}
1 & 0 & 0 \\
1+c_{3} & 2+2 c_{3} & 0 \\
1+c_{1}+c_{2} & c_{1} & 2+c_{1}+2 c_{2}
\end{array}\right),\left(\begin{array}{ccc}
1 & 0 & 0 \\
1+c_{3} & 2+2 c_{3} & 0 \\
c_{1} & 2 c_{1} & 1+2 c_{1}+c_{2}
\end{array}\right) \\
& \left(\begin{array}{ccc}
1 & 0 & 0 \\
0 & 1+c_{3} & 0 \\
0 & c_{1} & 1+c_{1}+c_{2}
\end{array}\right),\left(\begin{array}{ccc}
2 & 0 & 0 \\
0 & 1+c_{3} & 0 \\
0 & c_{1} & 1+c_{1}+c_{2}
\end{array}\right) \\
& \left(\begin{array}{ccc}
1 & 0 & 0 \\
0 & 1+c_{2} & 0 \\
1+c_{1}+c_{3} & 2 c_{1} & 2+2 c_{1}+2 c_{3}
\end{array}\right),\left(\begin{array}{ccc}
1 & 0 & 0 \\
0 & 1+c_{2} & 0 \\
2+c_{1}+c_{3} & 1+2 c_{1} & 4+2 c_{1}+2 c_{3}
\end{array}\right) \\
& \left(\begin{array}{ccc}
1 & 0 & 0 \\
0 & 1+c_{2} & 0 \\
1+c_{1}+c_{3} & 1+2 c_{1} & 2+2 c_{1}+2 c_{3}
\end{array}\right),\left(\begin{array}{ccc}
1 & 0 & 0 \\
0 & 1+c_{2} & 0 \\
2+c_{1}+c_{3} & 2+2 c_{1} & 4+2 c_{1}+2 c_{3}
\end{array}\right)
\end{aligned}
$$

where $c_{1}, c_{2}$, and $c_{3}$ can be any non-negative integers.

For the sake of brevity, we'll only consider the first $B_{N}$ family in equation 6.25 , to which the application of equation 3.11 yields the condition

$$
\begin{gathered}
\left(\begin{array}{ccc}
1 & 0 & 0 \\
-\frac{1+c_{3}}{2+2 c_{2}} & \frac{1}{2+2 c_{2}} & 0 \\
\frac{-2\left(1+c_{2}\right)^{2}+c_{1}\left(-1-2 c_{2}+c_{3}\right)}{\left(2+2 c_{2}\right)\left(2+c_{1}+2 c_{2}\right)} & -\frac{c_{1}}{\left(2+2 c_{2}\right)\left(2+c_{1}+2 c_{2}\right)} & \frac{1}{2+c_{1}+2 c_{2}}
\end{array}\right) p_{g} \\
*\left(\begin{array}{ccc}
1 & 0 & 0 \\
1+c_{3} & 2+2 c_{2} & 0 \\
1+c_{1}+c_{2} & c_{1} & 2+c_{1}+2 c_{2}
\end{array}\right) \in \mathbb{Z}^{3 \times 3}
\end{gathered}
$$


for each $p_{g} \in P_{G}$. Inserting each $p_{g}$ then yields

$$
\begin{gathered}
\left(\begin{array}{lll}
1 & 0 & 0 \\
0 & 1 & 0 \\
0 & 0 & 1
\end{array}\right),\left(\begin{array}{ccc}
-1 & 0 & 0 \\
\frac{2+c_{1}+c_{2}+c_{3}}{2+2 c_{2}} & \frac{c_{1}}{2+2 c_{2}} & \frac{2+c_{1}+2 c_{2}}{2+2 c_{2}} \\
\frac{\left(2-c_{1}+2 c_{2}\right)\left(2+c_{1}+c_{2}+c_{3}\right)}{\left(2+2 c_{2}\right)\left(2+c_{1}+2 c_{2}\right)} & \frac{2-c_{1}+2 c_{2}}{2+2 c_{2}} & -\frac{c_{1}}{2+2 c_{2}}
\end{array}\right), \\
\left(\begin{array}{ccc}
1 & 0 & 0 \\
\frac{1}{1+c_{3}} & 1 & 0 \\
\frac{2\left(1+c_{2}\right)^{2}+c_{2}\left(1+2 c_{2}-c_{3}\right)}{\left(1+c_{2}\right)\left(2+c_{1}+2 c_{2}\right)} & 0 & 1
\end{array}\right),\left(\begin{array}{ccc}
1 & 0 \\
\frac{c_{1}+c_{2}-c_{3}}{2+2 c_{2}} & \frac{c_{1}}{2+2 c_{2}} & \frac{2+c_{1}+2 c_{2}}{2+2 c_{2}} \\
-\frac{c_{1}+c_{2}-c_{3}}{2+2 c_{2}} & \frac{2-c_{1}+2 c_{2}}{2+2 c_{2}} & -\frac{c_{1}}{2+2 c_{2}}
\end{array}\right)
\end{gathered}
$$

which has five unique nontrivial conditions: $\left\{d_{1}=\frac{c_{1}}{2+2 c_{3}}, d_{2}=\frac{2+c_{1}+2 c_{2}}{2+2 c_{3}}, d_{3}=\frac{c_{1}+c_{2}-c_{3}}{2+2 c_{3}}, d_{4}=\right.$ $\left.\frac{\left(c_{1}+c_{2}-c_{3}\right)\left(2+c_{1}+2 c_{3}\right)}{\left(2+2 c_{3}\right)\left(2+c_{1}+2 c_{2}\right)}, d_{5}=\frac{c_{1}^{2}-\left(2+2 c_{3}\right)^{2}}{\left(2+2 c_{3}\right)\left(2+c_{1}+2 c_{2}\right)}\right\}$. Applying Mathematica's Reduce function under the assumption that all $c$ and $d$ variables are integers, we find that we can satisfy most of these conditions by breaking up $B_{N}$ into the following seven cases.

For the first case, we set $c_{1}=c_{2}=c_{3}=d_{1}=d_{3}=d_{4}=0$ and $d_{2}=d_{5}=0$. This simplifies the $B_{N}$ family to

$$
\left(\begin{array}{lll}
1 & 0 & 0 \\
1 & 2 & 0 \\
1 & 0 & 2
\end{array}\right) .
$$

For the second case, we set $c_{1}=c_{3}=0$ and $c_{2}=-1+d_{2}$ so that $d_{1}=0, d_{3}=\frac{-1+d_{2}}{2}, d_{4}=$ $\frac{-1+d_{2}}{2 d_{2}}$, and $d_{5}=\frac{1}{d_{2}}$. This simplifies the $B_{N}$ family to

$$
\left(\begin{array}{ccc}
1 & 0 & 0 \\
1 & 2 & 0 \\
d_{2} & 0 & 2 d_{2}
\end{array}\right)
$$

where $d_{2}$ can be any integer greater than or equal to 2 that satisfies $\left\{\frac{-1+d_{2}}{2}, \frac{1}{d_{2}}\right\} \in \mathbb{Z}$. By inspection, it can be seen that no possible values of $d_{2}$ that will simultaneously satisfy all of these conditions and so this particular $B_{N}$ family can be discarded. 
For the third case, we set $c_{1}=0$ and $c_{2}=c_{3}$ so that $d_{1}=d_{3}=d_{4}=0$ and $d_{2}=d_{5}=1$. Then we define $c_{1}[$ new $]=c_{3}[$ old $]-1$ so as to replace $c_{3}$ with $c_{1}+1$. This simplifies the $B_{N}$ family to

$$
\left(\begin{array}{ccc}
1 & 0 & 0 \\
2+c_{1} & 4+2 c_{1} & 0 \\
2+c_{1} & 0 & 4+2 c_{1}
\end{array}\right)
$$

where $c_{1} \geq 0$.

For the fourth case, we set $c_{1}=0$ and $c_{2}=-1+d_{2}+c_{3} d_{2}$ so that $d_{1}=0, d_{3}=\frac{-1+d_{2}}{2}, d_{4}=$ $\frac{-1+d_{2}}{2 d_{2}}$, and $d_{5}=\frac{1}{d_{2}}$. This simplifies the $B_{N}$ family to

$$
\left(\begin{array}{ccc}
1 & 0 & 0 \\
1+c_{3} & 2+2 c_{3} & 0 \\
\left(1+c_{3}\right) d_{2} & 0 & \left(2+2 c_{3}\right) d_{2}
\end{array}\right)
$$

where $c_{3}$, and $d_{2}$ are integers that satisfy $c_{3} d_{2}>0, d_{2} \geq 2$, and $\left\{\frac{-1+d_{2}}{2}, \frac{1}{d_{2}}\right\} \in \mathbb{Z}$. By inspection, it can be seen that there are no solutions to these conditions and thus this $B_{N}$ family can be discarded.

For the fifth case, we set $c_{1}=2 d_{1}$ and $c_{2}=c_{3}=0$ so that $d_{2}=1+d_{1}, d_{3}=d_{4}=d_{1}$, and $d_{5}=1-d_{1}$. Then we define $c_{1}[\mathrm{new}]=d_{1}[\mathrm{old}]-1$ so as to replace $d_{1}$ with $c_{1}+1$. This simplifies the $B_{N}$ family to

$$
\left(\begin{array}{ccc}
1 & 0 & 0 \\
1 & 2 & 0 \\
3+2 c_{1} & 2+2 c_{1} & 4+2 c_{1}
\end{array}\right)
$$

where $c_{1} \geq 0$.

For the sixth case, we set $c_{2}=c_{3}=\frac{c_{1}-2 d_{1}}{2 d_{1}}$ so that $d_{2}=1+d_{1}, d_{3}=d_{4}=d_{1}$, and $d_{5}=1-d_{1}$. This simplifies the $B_{N}$ family to

$$
\left(\begin{array}{ccc}
1 & 0 & 0 \\
\frac{c_{1}}{2 d_{1}} & \frac{c_{1}}{d_{1}} & 0 \\
c_{1}+\frac{c_{1}}{2 d_{1}} & c_{1} & c_{1}+\frac{c_{1}}{d_{1}}
\end{array}\right)
$$


where $c_{1}$ and $d_{1}$ are any integers that satisfy $c_{1}>2 d_{1}, d_{1} \geq 1$, and $\frac{c_{1}}{2 d_{1}} \in \mathbb{Z}$. Additional reduction is needed to bring this family into our prescribed form, which is

$$
\left(\begin{array}{ccc}
1 & 0 & 0 \\
2+c_{1} & 2\left(2+c_{1}\right) & 0 \\
\left(2+c_{1}\right)\left(3+2 c_{2}\right) & 2\left(2+c_{1}\right)\left(1+c_{2}\right) & 2\left(2+c_{1}\right)\left(2+c_{2}\right)
\end{array}\right)
$$

where $c_{1}$ and $c_{2}$ can be any non-negative integers. However, we choose to skip the details here because we instead want to focus more attention on the seventh case.

For the seventh and final case (to which the remainder of this section will be devoted), we set $c_{2}=\frac{-2 d_{1}-c_{1} d_{1}+c_{1} d_{2}}{2 d_{1}}$ and $c_{3}=\frac{c_{1}-2 d_{1}}{2 d_{1}}$ so that $d_{3}=\frac{-1+d_{1}+d_{2}}{2}, d_{4}=\frac{\left(1+d_{1}\right)\left(-1+d_{1}+d_{2}\right)}{2 d_{2}}$, and $d_{5}=\frac{1-d_{1}^{2}}{d_{2}}$. This simplifies the $B_{N}$ family to

$$
\left(\begin{array}{ccc}
1 & 0 & 0 \\
\frac{c_{1}}{2 d_{1}} & \frac{c_{1}}{d_{1}} & 0 \\
\frac{c_{1}\left(d_{1}+d_{2}\right)}{2 d_{1}} & c_{1} & \frac{c_{1} d_{2}}{d_{1}}
\end{array}\right)
$$

where $c_{1}, d_{1}$, and $d_{2}$ are any integers that satisfy $d_{2}>1+d_{1}, c_{1} \geq 2 d_{1}, d_{1} \geq 1$, and $\left\{\frac{\left(1+d_{1}\right)\left(-1+d_{1}+d_{2}\right)}{2 d_{1}}\right.$, $\left.\frac{1-d_{1}^{2}}{d_{2}}, \frac{c_{1}}{2 d_{1}}, \frac{-\left(2+c_{1}\right) d_{1}+c_{1} d_{2}}{2 d_{1}}, \frac{-1+d_{1}+d_{2}}{2}\right\} \in \mathbb{Z}$.

Here the quotients in $B_{N}$ are undesirable because each element must be integral. To simplify $B_{N}$, we can replace $d_{1}$ with $\frac{c_{1}}{2 g_{1}}$ which simplifies the $B_{N}$ family to

$$
\left(\begin{array}{ccc}
1 & 0 & 0 \\
d_{1} & 2 d_{1} & 0 \\
d_{1} d_{2}+d_{3} & 2 d_{3} & 2 d_{1} d_{2}
\end{array}\right)
$$

where, for convenience, $g_{1}$ and $c_{1}$ have been relabeled as $d_{1}$ and $d_{3}$, respectively, and $d_{1}, d_{2}$, and $d_{3}$ can be any integers that satisfy $d_{2}>1+\frac{d_{3}}{d_{1}}, d_{3} \geq \frac{d_{3}}{d_{1}}, \frac{d_{3}}{d_{1}} \geq 1$, and $\left\{\frac{\left(d_{1}+d_{3}\right)\left(d_{1}\left(-1+d_{2}\right)+d_{3}\right)}{4 d_{1}^{2} d_{2}}, \frac{d_{1}^{2}-d_{3}^{2}}{d_{1}^{2} d_{2}}\right.$, $\left.\frac{d_{1}\left(-1+d_{2}\right)+d_{3}}{2 d_{1}}, \frac{d_{3}}{d_{1}}\right\} \in \mathbb{Z}$.

This $B_{N}$ family is not ideal because the allowed ranges of values are dependent on other variables. To remedy this, we can again apply Mathematica's Reduce command which bifurcates 
the family into three subcases. The first subcase has $d_{1}=d_{3}=1$ and $d_{2} \geq 3$. The second has $d_{1}=1, d_{2} \geq 3, d_{3} \geq 2$, and $d_{2}>1+d_{3}$. The third has $d_{1} \geq 2, d_{2} \geq 3, d_{1}\left(-1+d_{2}\right)>d_{3}$, and $d_{3} \geq d_{1}$. Once again, for the sake of brevity, we will only explore the second subcase, which nicely illustrates some of the complexities encountered in producing Table A.1. For this subcase, $B_{N}$ simplifies to

$$
\left(\begin{array}{ccc}
1 & 0 & 0 \\
1 & 2 & 0 \\
d_{1}+d_{2} & 2 d_{2} & 2 d_{1}
\end{array}\right)
$$

where for convenience, $d_{2}$ and $d_{3}$ have been relabeled as $d_{1}$ and $d_{2}$, respectively, and $d_{1}$ and $d_{2}$ can be any integers that satisfy $d_{1} \geq 3, d_{2} \geq 2, d_{1}>1+d_{2}$ and $\left\{\frac{\left(1+d_{2}\right)\left(-1+d_{1}+d_{2}\right)}{2 d_{1}}, \frac{-1+d_{2}^{2}}{d_{1}}\right.$, $\left.\frac{-1+d_{1}+d_{2}}{2}\right\} \in \mathbb{Z}$.

The extra condition $\frac{-1+d_{1}+d_{2}}{2} \in \mathbb{Z}$ can be eliminated if we define $k_{1}=\frac{-1+d_{1}+d_{2}}{2}$ and then replace each occurrence of $d_{2}$ with $1-d_{1}+2 k_{1}$. This substitution simplifies the $B_{N}$ family to

$$
\left(\begin{array}{ccc}
1 & 0 & 0 \\
1 & 2 & 0 \\
1+2 d_{2} & 2-2 d_{1}+4 d_{2} & 2 d_{1}
\end{array}\right)
$$

where $k_{1}$ has been relabeled $d_{2}$ [new] for convenience, and $d_{1}$ and $d_{2}$ can be any integers that satisfy $d_{1} \geq 3,2 d_{2} \geq 1+d_{1}, d_{1}>1+d_{2}$ and $\frac{2\left(d_{2}+d_{2}^{2}\right)}{d_{1}} \in \mathbb{Z}$.

While the extra conditions are looking much cleaner, the ranges of the variables have grown significantly more convoluted. To remedy this, we again use Mathematica's Reduce command which replaces $d_{1}$ with $5+2 d_{1}+d_{2}$ and $d_{2}$ with $3+d_{1}+d_{2}$. This simplifies the $B_{N}$ family to

$$
\left(\begin{array}{ccc}
1 & 0 & 0 \\
1 & 2 & 0 \\
7+2 d_{1}+2 d_{2} & 4+2 d_{2} & 10+4 d_{1}+2 d_{2}
\end{array}\right)
$$

where $d_{1}$ and $d_{2}$ can be any non-negative integers that satisfy $\frac{2\left(2+3 d_{1}+d_{1}^{2}\right)}{5+2 d_{1}+d_{2}} \in \mathbb{Z}$. 
We only followed the $2^{\text {nd }}$ subcase of the $7^{\text {th }}$ case bifurcated from the $1^{\text {st }} B_{N}$ family from the application of equation 3.9. For $G=A m m 2(\mathrm{ACC} \# 15)$ and $P_{N}=m_{x}$, other threads were similarly complicated. Hence the extraordinary number of unique $B_{N}$ families associated with this case, as seen in Table 5.1. 


\section{Chapter 7}

\section{Conclusions and Future Work}

This thesis contains several key results. First, the cpg-normal subgroups for each crystallographic space group (i.e. normal subgroups of the point group of a space group) have been tabulated and the corresponding abstract quotient groups have been calculated. Second, we established two conditions that a sublattice $T_{N}$ of crystallographic space group $G$ must satisfy in order to support extensions with cpg-normal subgroup $P_{N}$ that are normal subgroups of $G$. Third, an algorithm to find these normally supportive sublattices has been developed, along with Mathematica code that implements the algorithm. The algorithm first applies the simpler condition (inclusion, equation 3.9) to narrow the range of candidate sublattices, and then applies the more difficult condition ( $P_{G}$-invariance, equation 3.11$)$ to each sublattice that met the first condition. Fourth, the normally supportive sublattices only depend on the lattice centering type of the parent space group $G$ and the cpg-normal subgroup $P_{N}$ of the point group $P_{G}$ of $G$; we found that there are only 129 unique combinations of parent lattice centering and cpg-normal subgroup that need to be studied.

Fifth, we applied our code to determine and tabulate all of the normally supportive sublattices for each of the cpg-normal subgroups $P_{N}$ of each of the 230 crystallographic space groups, excepting those cases for which $P_{N}$ was the trivial subgroup 1 (these cases were too complicated with the present approach). Because space groups of the same arithmetic class have the same 
cpg-normal subgroups, this analysis was only performed for the unique symmorphic space group from each arithmetic crystal class. Upon applying the inclusion condition (equation 3.9) to the 129 input scenarios, only 41 unique inclusion sublattices emerged. The $P_{G}$-invariance test (equation 3.11) required only the point-group operators of $P_{G}$ and the unique inclusion sublattice associated with $P_{N} \subseteq P_{G}$. For a given arithemetic class and cpg-normal subgroup, the basis vectors of each normally-supportive sublattice are represented symbolically in Table A.1 by the columns of a $B_{N}$ matrix in Hermite normal form. With considerable computer-guided algebraic manipulation, we were able to bring all but a handful of the $B_{N}$ matrices into a standard form in which all variables have integer values, all variable ranges extend from zero to infinity, and no matrix elements contain quotients; however, some cases do include additional conditions on the variables (a quotient expression that is required to be integral). Four cases had slightly more complicated variable ranges. A matrix $B_{N}$ containing symbolic variables actually represents an infinite family of sublattices, and in most cases, the complete set of normally-supportive sublattices could only be described using multiple $B_{N}$ matrices that each represent multiple disjoint subfamilies. The most complicated case required 77 such subfamilies. A total of 1773 such $B_{N}$ families are presented in Table A.1. The algebraic manipulation required to achieve the nice standard form was the most difficult aspect of this research. Of particular interest, a paper by Litvin and Kopský in 2000 claimed that cubic space groups will only have a finite number of normal subgroups [9]. Our results support this claim as there are no infinite $B_{N}$ families corresponding to any of the cubic space groups (arithmetic crystal classes 59-73).

The ultimate goal of this research is to determine, in general, the types of topological defects that can be formed by the intersection of domain boundaries in crystals. During a crystal-crystal phase transition involving a loss of symmetry, domains of the low-symmetry phase form and eventually fill the volume of the crystal. The cosets of the child symmetry group $H$ of the low-symmetry phase in the parent symmetry group $G$ of the high-symmetry phase are acted upon by the symme- 
tries of the quotient group $Q=G / N$, where $N$ is the normal core of $H$ in $G$. This action of $Q$ on these cosets generates a coset graph, the topology of which appears to be relevant to the defecttopology problem.

Now that the normally supportive sublattices have been tabulated, the broader vision of understanding and possibly classifying the topologies of domain-interface defects will require additional work. The first step will be the group extension problem, or in other words combining $P_{N}$ and $T_{N}$ to obtain subgroups $N$ of $G$ (i.e. space groups in the same arithmetic crystal class as $T_{N} \rtimes P_{N}$ ) [31]. The second step will be to enforce the normality condition $N \unlhd G$ to obtain the csg-normal subgroups of $G$. Once the csg-normal subgroups of $G$ are known, the third step will be to tabulate quotient groups $Q=G / N$ for each one. Fourth, a general strategy will be needed for generating the coset graph of a given $Q$ and using it to deduce the defect-interface topologies that might result. By coset graph, we mean a complete colored digraph, wherein the vertices are the cosets of $N$ in $G$, the arcs are ordered vertex pairs and the quotient group $Q$ is an externally-defined vertex-transitive automorphism group whose action on the arcs assigns distinct colors to distinct arc orbits [32,33]. Figure 1.5 is an example of a coset graph with $G=P 6_{3} / m m c(\# 194)$ and $N=P 3 c(\# 158)$.

Though difficult, the determination of normally-supportive sublattices was a necessary step in identifying and classifying the csg-normal subgroups of crystallographic space groups. The results should be useful in future work that aims to understand the topology of domain-intersection defects in crystals. After this work has been published, the code and relevant data files will be made available in a public Github repository. 


\section{Bibliography}

[1] J. A. Gallian, Contemporary Abstract Algebra, 7th ed. (Brooks/Cole Cengage Learning, Belmont, CA, 2010).

[2] J. R. Durbin, Modern Algebra: An Introduction (John Wiley \& Sons, New York, 2008).

[3] M. S. Dresselhaus, G. Dresselhaus, and A. Jorio, Group Theory: Application to the Physics of Condensed Matter (Springer, Heidelberg, Germany, 2008).

[4] S. Hassani, Mathematical Physics: A Modern Introduction to Its Foundations, 2nd ed. (Springer, Switzerland, 2013).

[5] E. Koch, W. Fisher, and U. Müller, "Normalizers of Space Groups and their use in Crystallography,", M. I. Aroyo ed., (2016), International Tables for Crystallography A, Space Group Symmetry, Chap. 3.5.1.

[6] M. Gubler, "Normalizer Groups and Automorphism Groups of Symmetry Groups," Zeitschrift für Kristallographie 158, 1-26 (1982).

[7] E. Koch and W. Fisher, "Examples of the use of Normalizers,", M. I. Aroyo ed., (2016), International Tables for Crystallography A, Space Group Symmetry, Chap. 3.5.3. 
[8] E. Koch and U. Müller, "Euklidische Normalisatoren für trikline und monokline Raumgruppen bei spezieller Metrik des Translationengitters,” Acta Crystallographica A46, 826-831 (1990).

[9] D. B. Litvin and V. Kopský, "Subperiodic Groups Isomorphic to Factor Groups of Reducible Space Groups," Acta Crystallographica A56, 370-374 (2000).

[10] V. Kopský and D. B. Litvin, "Scanning of Space Groups,", (2010), International Tables for Crystallography E, Subperiodic Groups, Chap. 5.

[11] J. D. Jarratt, "The decomposition of crystal families," Mathematical Proceedings of the Cambridge Philosophical Society 88, 245-263 (1980).

[12] V. Kopský, "The Use of Subdirect and Multiple Subdirect Products or Sums in Crystallography," Czechoslovak Journal of Physics D 26, 945-967 (1988).

[13] V. Kopský, "Reducible Space Groups," Proceedings from the 16th International Colloquium on Group Theoretical Methods in Physics 313, 352-356 (1988).

[14] V. Janovec and V. Kopský, "Layer Groups, Scanning Tables and the Structure of Domain Walls," Ferroelectrics 191, 23-28 (1997).

[15] V. Janovec, M. Grocký, V. Kopský, and Z. Kluiber, "On Atomic Displacements in 90 Degree Ferroelectric Domain Walls of Tetragonal $\mathrm{BaTiO}_{3}$ Crystals," Ferroelectrics 303, 65-68 (2004).

[16] J. Fuksa and V. Janovec, "Macroscopic symmetries and domain configurations of engineered domain structures," Journal of Physics: Condensed Matter pp. 3795-3812 (2002). 
[17] D. M. Hatch, H. T. Stokes, and W. Cao, "Allowed Mesoscopic Point Group Symmetries in Domain Average Engineering of Perovskite Ferroelectric Crystals," Journal of Applied Physics 94, 5220-5227 (2003).

[18] H. T. Stokes and D. M. Hatch, "Symmetry of Possible Average Multidomain Structures at a Phase Transition," Ferroelectrics 292, 59-63 (2003).

[19] H. T. Stokes, D. M. Hatch, and B. J. Campbell, "Isotropy Software Suite," iso.byu.edu .

[20] V. Kopský, "Lattices of Normal Subgroups of Space and Subperiodic Groups: A Scouting View of the Problem," Czechoslovak Journal of Physics B 37, 785-808 (1987).

[21] V. Kopský, "Towards a System in Space Group Representations," Computers and Mathematics with Applications 16, 493-505 (1988).

[22] J. Fuksa and V. Kopský, "Isomorphism of lattices of subgroups of the layer and rod groups with sublattices of subgroups of the space groups," Acta Crystallographica Section A Foundations of Crystallography A67, 87-92 (2010).

[23] A. S. Gibbs, K. S. Knight, and P. Lightfoot, "The High Temperature Phase Transitions of Hexagonal YMnO3," Physical Review B 83, 1-9 (2011).

[24] M. Fiebig, T. Lottermoser, D. Fröhlich, A. V. Goltsev, and R. V. Pisarev, "Observation of coupled magnetic and electric domains," Nature 419, 818-820 (2002).

[25] Q. Zhang, G. Tan, L. Gu, Y. Yao, C. Jin, Y. Wang, X. Duan, and R. Yu, "Direct Observation of Multiferroic Vortex Domains in $\mathrm{YMnO}_{3}$," Nature pp. 1-5 (2013).

[26] S. Cheng, D. Zhang, S. Deng, X. Li, J. Li, G. Tan, Y. Zhu, and J. Zhu, "Domain Configurations in Dislocations Embedded Hexagonal Manganite Systems: From the View of Graph Theory," Applied Physics Letters 112, 1-5 (2018). 
[27] M. Mostovoy, “A whirlwind of opportunities," Nature Materials 9, 188-190 (2010).

[28] A. P. Cracknell, J. Lorenc, and J. A. Przystawa, "Landau's theory of second-order phase transitions and its application to ferromagnetism," Journal of Physics C: Solid State Physics 9, 1731-1758 (1976).

[29] T. Hahn, H. Klapper, U. Müller, and M. I. Aroyo, "Point Groups and Crystal Classes,", (2016), International Tables for Crystallography A, Space Group Symmetry, Chap. 3.2.

[30] W. S. Morgan and G. L. W. Hart, Private communication (2018).

[31] E. Ascher and A. Janner, "Algebraic Aspects of Crystallography: Space Groups as Extensions," Helvetica Physica Acta 38, 551-572 (1965).

[32] R. Dirl and B. L. Davies, "Space Group Subgroups generated by Sublattice Relations: Software for IBM-Compatible PCs," World Scientific: Symmetry and Structural Properties of Condensed Matter, Proceeding of the Third International School on Theoretical Physics pp. 338-341 (1995).

[33] B. L. Davies, R. Dirl, P. Zeiner, and V. Janovec, "Space Group Coset Decompositions: Software and Applications to Phase Transitions," World Scientific: Symmetry and Structural Properties of Condensed Matter, Proceeding of the Third International School on Theoretical Physics pp. 342-345 (1995). 


\section{Appendix A}

\section{Normally Supportive Sublattices}

Table A.1 A table of normally supportive sublattices of crystallographic space groups ordered by arithmetic crystal class and cpg-normal subgroup. The first column is the arithmetic class number and crystallographic space group representative. The second column is the cpg-normal subgroup $P_{N}$ with a number and a name. The cpg-normal subgroup 1 in each case is omitted from this table for reasons explained in Chapter 3. The third column is the HNF matrix that represents a normally supportive sublattice laid out row by row. These matrices are numbered for convenience. The fourth column is an index (c\#) which identifies additional conditions on the $d$ parameters in $B_{N}$ (rational polynomial functions that must have integer values) from Table A.2. An asterisk next to the $\mathrm{c} \#$ denotes conditions that have no possible solutions. When no $\mathrm{c \#}$ is provided, the variables can take on any non-negative integer values.

\begin{tabular}{l|l|l|l} 
ACC & $P_{N}$ & $B_{N}$ matrix row by row & c\# \\
\hline $2 P \overline{1}$ & $2 \overline{1}$ & $1\{\{1,0,0\},\{0,1,0\},\{0,0,1\}\}$ & \\
$2 P \overline{1}$ & $2 \overline{1}$ & $2\{\{1,0,0\},\{0,1,0\},\{1,1,2\}\}$ & \\
$2 P \overline{1}$ & $2 \overline{1}$ & $3\{\{1,0,0\},\{0,1,0\},\{1,0,2\}\}$ & \\
$2 P \overline{1}$ & $2 \overline{1}$ & $4\{\{1,0,0\},\{0,1,0\},\{0,1,2\}\}$ & \\
$2 P \overline{1}$ & $2 \overline{1}$ & $5\{\{1,0,0\},\{0,1,0\},\{0,0,2\}\}$ & \\
$2 P \overline{1}$ & $2 \overline{1}$ & $6\{\{1,0,0\},\{1,2,0\},\{0,0,1\}\}$ & \\
$2 P \overline{1}$ & $2 \overline{1}$ & $7\{\{1,0,0\},\{0,2,0\},\{0,0,1\}\}$ & \\
$2 P \overline{1}$ & $2 \overline{1}$ & $8\{\{1,0,0\},\{1,2,0\},\{1,0,2\}\}$ & \\
\hline
\end{tabular}


Table A.1 - continued from previous page

\begin{tabular}{|c|c|c|c|}
\hline ACC & $P_{N}$ & $B_{N}$ matrix row by row & $\mathrm{c \#}$ \\
\hline $2 P \overline{1}$ & $2 \overline{1}$ & $9\{\{1,0,0\},\{1,2,0\},\{0,0,2\}\}$ & \\
\hline $2 P \overline{1}$ & $2 \overline{1}$ & $10\{\{1,0,0\},\{0,2,0\},\{1,0,2\}\}$ & \\
\hline $2 P \overline{1}$ & $2 \overline{1}$ & $11\{\{1,0,0\},\{0,2,0\},\{0,0,2\}\}$ & \\
\hline $2 P \overline{1}$ & $2 \overline{1}$ & $12\{\{2,0,0\},\{0,1,0\},\{0,0,1\}\}$ & \\
\hline $2 P \overline{1}$ & $2 \overline{1}$ & $13\{\{2,0,0\},\{0,1,0\},\{0,1,2\}\}$ & \\
\hline $2 P \overline{1}$ & $2 \overline{1}$ & $14\{\{2,0,0\},\{0,1,0\},\{0,0,2\}\}$ & \\
\hline $2 P \overline{1}$ & $2 \overline{1}$ & $15\{\{2,0,0\},\{0,2,0\},\{0,0,1\}\}$ & \\
\hline $2 P \overline{1}$ & $2 \overline{1}$ & $16\{\{2,0,0\},\{0,2,0\},\{0,0,2\}\}$ & \\
\hline $3 P 2$ & $22 z$ & $1\left\{\{1,0,0\},\{0,1,0\},\left\{1+c_{1}, 1+c_{1}, 2+2 c_{1}\right\}\right\}$ & \\
\hline $3 P 2$ & $22_{z}$ & $2\left\{\{1,0,0\},\{0,1,0\},\left\{1+c_{1}, 0,2+2 c_{1}\right\}\right\}$ & \\
\hline $3 P 2$ & $22_{z}$ & $3\left\{\{1,0,0\},\{0,1,0\},\left\{0,1+c_{1}, 2+2 c_{1}\right\}\right\}$ & \\
\hline $3 P 2$ & $22_{z}$ & $4\left\{\{1,0,0\},\{0,1,0\},\left\{0,0,1+c_{1}\right\}\right\}$ & \\
\hline $3 P 2$ & $22 z$ & $5\left\{\{1,0,0\},\{1,2,0\},\left\{1+c_{1}, 0,2+2 c_{1}\right\}\right\}$ & \\
\hline $3 P 2$ & $22 z$ & $6\left\{\{1,0,0\},\{1,2,0\},\left\{0,0,1+c_{1}\right\}\right\}$ & \\
\hline $3 P 2$ & $22 z$ & $7\left\{\{1,0,0\},\{0,2,0\},\left\{1+c_{1}, 0,2+2 c_{1}\right\}\right\}$ & \\
\hline $3 P 2$ & $22 z$ & $8\left\{\{1,0,0\},\{0,2,0\},\left\{0,0,1+c_{1}\right\}\right\}$ & \\
\hline $3 P 2$ & $22 z$ & $9\left\{\{2,0,0\},\{0,1,0\},\left\{0,1+c_{1}, 2+2 c_{1}\right\}\right\}$ & \\
\hline $3 P 2$ & $22 z$ & $10\left\{\{2,0,0\},\{0,1,0\},\left\{0,0,1+c_{1}\right\}\right\}$ & \\
\hline $3 P 2$ & $22 z$ & $11\left\{\{2,0,0\},\{0,2,0\},\left\{0,0,1+c_{1}\right\}\right\}$ & \\
\hline $4 C 2$ & $22 z$ & $1\left\{\{1,0,0\},\{0,1,0\},\left\{1+c_{1}, 1+c_{1}, 2+2 c_{1}\right\}\right\}$ & \\
\hline $4 C 2$ & $22 z$ & $2\left\{\{1,0,0\},\{0,1,0\},\left\{1+c_{1}, 0,2+2 c_{1}\right\}\right\}$ & \\
\hline $4 C 2$ & $22 z$ & $3\left\{\{1,0,0\},\{0,1,0\},\left\{0,1+c_{1}, 2+2 c_{1}\right\}\right\}$ & \\
\hline $4 C 2$ & $22 z$ & $4\left\{\{1,0,0\},\{0,1,0\},\left\{0,0,1+c_{1}\right\}\right\}$ & \\
\hline $4 C 2$ & $22 z$ & $5\left\{\{1,0,0\},\{1,2,0\},\left\{1+c_{1}, 0,2+2 c_{1}\right\}\right\}$ & \\
\hline $4 C 2$ & $22_{z}$ & $6\left\{\{1,0,0\},\{1,2,0\},\left\{0,0,1+c_{1}\right\}\right\}$ & \\
\hline $4 C 2$ & $22 z$ & $7\left\{\{1,0,0\},\{0,2,0\},\left\{1+c_{1}, 0,2+2 c_{1}\right\}\right\}$ & \\
\hline $4 C 2$ & $22 z$ & $8\left\{\{1,0,0\},\{0,2,0\},\left\{0,0,1+c_{1}\right\}\right\}$ & \\
\hline $4 C 2$ & $22 z$ & $9\left\{\{2,0,0\},\{0,1,0\},\left\{0,1+c_{1}, 2+2 c_{1}\right\}\right\}$ & \\
\hline
\end{tabular}


Table A.1 - continued from previous page

\begin{tabular}{|c|c|c|c|}
\hline ACC & $P_{N}$ & $B_{N}$ matrix row by row & $\mathrm{c \#}$ \\
\hline $4 C 2$ & $22_{z}$ & $10\left\{\{2,0,0\},\{0,1,0\},\left\{0,0,1+c_{1}\right\}\right\}$ & \\
\hline $4 C 2$ & $22 z$ & $11\left\{\{2,0,0\},\{0,2,0\},\left\{0,0,1+c_{1}\right\}\right\}$ & \\
\hline $5 \mathrm{Pm}$ & $2 m_{z}$ & $1\left\{\left\{1+c_{1}, 0,0\right\},\left\{c_{2}, 1+c_{2}+c_{3}, 0\right\},\{0,0,1\}\right\}$ & \\
\hline $5 \mathrm{Pm}$ & $2 m_{z}$ & $2\left\{\left\{1+c_{1}, 0,0\right\},\left\{c_{2}, 1+c_{2}+c_{3}, 0\right\},\{0,0,2\}\right\}$ & \\
\hline $5 \mathrm{Pm}$ & $2 m_{z}$ & $3\left\{\left\{1+c_{1}, 0,0\right\},\left\{c_{2}, 1+c_{2}+c_{3}, 0\right\},\{0,1,2\}\right\}$ & \\
\hline $5 \mathrm{Pm}$ & $2 m_{z}$ & $4\left\{\left\{1+c_{1}, 0,0\right\},\left\{c_{2}, 1+c_{2}+c_{3}, 0\right\},\{1,0,2\}\right\}$ & \\
\hline $5 \mathrm{Pm}$ & $2 m_{z}$ & $5\left\{\left\{1+c_{1}, 0,0\right\},\left\{c_{2}, 1+c_{2}+c_{3}, 0\right\},\{1,1,2\}\right\}$ & \\
\hline $6 \mathrm{Cm}$ & $2 m_{z}$ & $1\left\{\left\{1+c_{1}, 0,0\right\},\left\{c_{2}, 1+c_{2}+c_{3}, 0\right\},\{0,0,1\}\right\}$ & \\
\hline $6 \mathrm{Cm}$ & $2 m_{z}$ & $2\left\{\left\{1+c_{1}, 0,0\right\},\left\{c_{2}, 1+c_{2}+c_{3}, 0\right\},\{0,0,2\}\right\}$ & \\
\hline $6 \mathrm{Cm}$ & $2 m_{z}$ & $3\left\{\left\{1+c_{1}, 0,0\right\},\left\{c_{2}, 1+c_{2}+c_{3}, 0\right\},\{0,1,2\}\right\}$ & \\
\hline $6 \mathrm{Cm}$ & $2 m_{z}$ & $4\left\{\left\{1+c_{1}, 0,0\right\},\left\{c_{2}, 1+c_{2}+c_{3}, 0\right\},\{1,0,2\}\right\}$ & \\
\hline $6 \mathrm{Cm}$ & $2 m_{z}$ & $5\left\{\left\{1+c_{1}, 0,0\right\},\left\{c_{2}, 1+c_{2}+c_{3}, 0\right\},\{1,1,2\}\right\}$ & \\
\hline $7 P 2 / m$ & $2 \overline{1}$ & $1\{\{1,0,0\},\{0,1,0\},\{0,0,1\}\}$ & \\
\hline $7 P 2 / m$ & $2 \overline{1}$ & $2\{\{1,0,0\},\{0,1,0\},\{1,1,2\}\}$ & \\
\hline $7 P 2 / m$ & $2 \overline{1}$ & $3\{\{1,0,0\},\{0,1,0\},\{1,0,2\}\}$ & \\
\hline $7 P 2 / m$ & $2 \overline{1}$ & $4\{\{1,0,0\},\{0,1,0\},\{0,1,2\}\}$ & \\
\hline $7 P 2 / m$ & $2 \overline{1}$ & $5\{\{1,0,0\},\{0,1,0\},\{0,0,2\}\}$ & \\
\hline $7 P 2 / m$ & $2 \overline{1}$ & $6\{\{1,0,0\},\{1,2,0\},\{0,0,1\}\}$ & \\
\hline $7 P 2 / m$ & $2 \overline{1}$ & $7\{\{1,0,0\},\{0,2,0\},\{0,0,1\}\}$ & \\
\hline $7 P 2 / m$ & $2 \overline{1}$ & $8\{\{1,0,0\},\{1,2,0\},\{1,0,2\}\}$ & \\
\hline $7 P 2 / m$ & $2 \overline{1}$ & $9\{\{1,0,0\},\{1,2,0\},\{0,0,2\}\}$ & \\
\hline $7 P 2 / m$ & $2 \overline{1}$ & $10\{\{1,0,0\},\{0,2,0\},\{1,0,2\}\}$ & \\
\hline $7 P 2 / m$ & $2 \overline{1}$ & $11\{\{1,0,0\},\{0,2,0\},\{0,0,2\}\}$ & \\
\hline $7 P 2 / m$ & $2 \overline{1}$ & $12\{\{2,0,0\},\{0,1,0\},\{0,0,1\}\}$ & \\
\hline $7 P 2 / m$ & $2 \overline{1}$ & $13\{\{2,0,0\},\{0,1,0\},\{0,1,2\}\}$ & \\
\hline $7 P 2 / m$ & $2 \overline{1}$ & $14\{\{2,0,0\},\{0,1,0\},\{0,0,2\}\}$ & \\
\hline $7 P 2 / m$ & $2 \overline{1}$ & $15\{\{2,0,0\},\{0,2,0\},\{0,0,1\}\}$ & \\
\hline $7 P 2 / m$ & $2 \overline{1}$ & $16\{\{2,0,0\},\{0,2,0\},\{0,0,2\}\}$ & \\
\hline
\end{tabular}


Table A.1 - continued from previous page

\begin{tabular}{|c|c|c|c|}
\hline ACC & $P_{N}$ & $B_{N}$ matrix row by row & $\mathrm{c} \#$ \\
\hline $7 P 2 / m$ & $32 z$ & $1\left\{\{1,0,0\},\{0,1,0\},\left\{1+c_{1}, 1+c_{1}, 2+2 c_{1}\right\}\right\}$ & \\
\hline $7 P 2 / m$ & $32 z$ & $2\left\{\{1,0,0\},\{0,1,0\},\left\{1+c_{1}, 0,2+2 c_{1}\right\}\right\}$ & \\
\hline $7 P 2 / m$ & $32 z$ & $3\left\{\{1,0,0\},\{0,1,0\},\left\{0,1+c_{1}, 2+2 c_{1}\right\}\right\}$ & \\
\hline $7 P 2 / m$ & $32 z$ & $4\left\{\{1,0,0\},\{0,1,0\},\left\{0,0,1+c_{1}\right\}\right\}$ & \\
\hline $7 P 2 / m$ & $32 z_{z}$ & $5\left\{\{1,0,0\},\{1,2,0\},\left\{1+c_{1}, 0,2+2 c_{1}\right\}\right\}$ & \\
\hline $7 P 2 / m$ & $32 z$ & $6\left\{\{1,0,0\},\{1,2,0\},\left\{0,0,1+c_{1}\right\}\right\}$ & \\
\hline $7 P 2 / m$ & $32 z$ & $7\left\{\{1,0,0\},\{0,2,0\},\left\{1+c_{1}, 0,2+2 c_{1}\right\}\right\}$ & \\
\hline $7 P 2 / m$ & $32 z$ & $8\left\{\{1,0,0\},\{0,2,0\},\left\{0,0,1+c_{1}\right\}\right\}$ & \\
\hline $7 P 2 / m$ & $32 z$ & $9\left\{\{2,0,0\},\{0,1,0\},\left\{0,1+c_{1}, 2+2 c_{1}\right\}\right\}$ & \\
\hline $7 P 2 / m$ & $32 z$ & $10\left\{\{2,0,0\},\{0,1,0\},\left\{0,0,1+c_{1}\right\}\right\}$ & \\
\hline $7 P 2 / m$ & $32 z$ & $11\left\{\{2,0,0\},\{0,2,0\},\left\{0,0,1+c_{1}\right\}\right\}$ & \\
\hline $7 P 2 / m$ & $4 m_{z}$ & $1\left\{\left\{1+c_{1}, 0,0\right\},\left\{c_{2}, 1+c_{2}+c_{3}, 0\right\},\{0,0,1\}\right\}$ & \\
\hline $7 P 2 / m$ & $4 m_{z}$ & $2\left\{\left\{1+c_{1}, 0,0\right\},\left\{c_{2}, 1+c_{2}+c_{3}, 0\right\},\{0,0,2\}\right\}$ & \\
\hline $7 P 2 / m$ & $4 m_{z}$ & $3\left\{\left\{1+c_{1}, 0,0\right\},\left\{c_{2}, 1+c_{2}+c_{3}, 0\right\},\{0,1,2\}\right\}$ & \\
\hline $7 P 2 / m$ & $4 m_{z}$ & $4\left\{\left\{1+c_{1}, 0,0\right\},\left\{c_{2}, 1+c_{2}+c_{3}, 0\right\},\{1,0,2\}\right\}$ & \\
\hline $7 P 2 / m$ & $4 m_{z}$ & $5\left\{\left\{1+c_{1}, 0,0\right\},\left\{c_{2}, 1+c_{2}+c_{3}, 0\right\},\{1,1,2\}\right\}$ & \\
\hline $7 P 2 / m$ & $52 / m_{z}$ & $1\{\{1,0,0\},\{0,1,0\},\{0,0,1\}\}$ & \\
\hline $7 P 2 / m$ & $52 / m_{z}$ & $2\{\{1,0,0\},\{0,1,0\},\{1,1,2\}\}$ & \\
\hline $7 P 2 / m$ & $52 / m_{z}$ & $3\{\{1,0,0\},\{0,1,0\},\{1,0,2\}\}$ & \\
\hline $7 P 2 / m$ & $52 / m_{z}$ & $4\{\{1,0,0\},\{0,1,0\},\{0,1,2\}\}$ & \\
\hline $7 P 2 / m$ & $52 / m_{z}$ & $5\{\{1,0,0\},\{0,1,0\},\{0,0,2\}\}$ & \\
\hline $7 P 2 / m$ & $52 / m_{z}$ & $6\{\{1,0,0\},\{1,2,0\},\{0,0,1\}\}$ & \\
\hline $7 P 2 / m$ & $52 / m_{z}$ & $7\{\{1,0,0\},\{0,2,0\},\{0,0,1\}\}$ & \\
\hline $7 P 2 / m$ & $52 / m_{z}$ & $8\{\{1,0,0\},\{1,2,0\},\{1,0,2\}\}$ & \\
\hline $7 P 2 / m$ & $52 / m_{z}$ & $9\{\{1,0,0\},\{1,2,0\},\{0,0,2\}\}$ & \\
\hline $7 P 2 / m$ & $52 / m_{z}$ & $10\{\{1,0,0\},\{0,2,0\},\{1,0,2\}\}$ & \\
\hline $7 P 2 / m$ & $52 / m_{z}$ & $11\{\{1,0,0\},\{0,2,0\},\{0,0,2\}\}$ & \\
\hline $7 P 2 / m$ & $52 / m_{z}$ & $12\{\{2,0,0\},\{0,1,0\},\{0,0,1\}\}$ & \\
\hline
\end{tabular}


Table A.1 - continued from previous page

\begin{tabular}{|c|c|c|c|}
\hline ACC & $P_{N}$ & $B_{N}$ matrix row by row & $\mathrm{c \#}$ \\
\hline $7 P 2 / m$ & $52 / m_{z}$ & $13\{\{2,0,0\},\{0,1,0\},\{0,1,2\}\}$ & \\
\hline $7 P 2 / m$ & $52 / m_{z}$ & $14\{\{2,0,0\},\{0,1,0\},\{0,0,2\}\}$ & \\
\hline $7 P 2 / m$ & $52 / m_{z}$ & $15\{\{2,0,0\},\{0,2,0\},\{0,0,1\}\}$ & \\
\hline $7 P 2 / m$ & $52 / m_{z}$ & $16\{\{2,0,0\},\{0,2,0\},\{0,0,2\}\}$ & \\
\hline $8 C 2 / m$ & $2 \overline{1}$ & $1\{\{1,0,0\},\{0,1,0\},\{0,0,1\}\}$ & \\
\hline $8 C 2 / m$ & $2 \overline{1}$ & $2\{\{1,0,0\},\{0,1,0\},\{1,1,2\}\}$ & \\
\hline $8 C 2 / m$ & $2 \overline{1}$ & $3\{\{1,0,0\},\{0,1,0\},\{1,0,2\}\}$ & \\
\hline $8 C 2 / m$ & $2 \overline{1}$ & $4\{\{1,0,0\},\{0,1,0\},\{0,1,2\}\}$ & \\
\hline $8 C 2 / m$ & $2 \overline{1}$ & $5\{\{1,0,0\},\{0,1,0\},\{0,0,2\}\}$ & \\
\hline $8 C 2 / m$ & $2 \overline{1}$ & $6\{\{1,0,0\},\{1,2,0\},\{0,0,1\}\}$ & \\
\hline $8 C 2 / m$ & $2 \overline{1}$ & $7\{\{1,0,0\},\{0,2,0\},\{0,0,1\}\}$ & \\
\hline $8 C 2 / m$ & $2 \overline{1}$ & $8\{\{1,0,0\},\{1,2,0\},\{1,0,2\}\}$ & \\
\hline $8 C 2 / m$ & $2 \overline{1}$ & $9\{\{1,0,0\},\{1,2,0\},\{0,0,2\}\}$ & \\
\hline $8 C 2 / m$ & $2 \overline{1}$ & $10\{\{1,0,0\},\{0,2,0\},\{1,0,2\}\}$ & \\
\hline $8 C 2 / m$ & $2 \overline{1}$ & $11\{\{1,0,0\},\{0,2,0\},\{0,0,2\}\}$ & \\
\hline $8 C 2 / m$ & $2 \overline{1}$ & $12\{\{2,0,0\},\{0,1,0\},\{0,0,1\}\}$ & \\
\hline $8 C 2 / m$ & $2 \overline{1}$ & $13\{\{2,0,0\},\{0,1,0\},\{0,1,2\}\}$ & \\
\hline $8 C 2 / m$ & $2 \overline{1}$ & $14\{\{2,0,0\},\{0,1,0\},\{0,0,2\}\}$ & \\
\hline $8 C 2 / m$ & $2 \overline{1}$ & $15\{\{2,0,0\},\{0,2,0\},\{0,0,1\}\}$ & \\
\hline $8 C 2 / m$ & $2 \overline{1}$ & $16\{\{2,0,0\},\{0,2,0\},\{0,0,2\}\}$ & \\
\hline $8 C 2 / m$ & $32 z$ & $1\left\{\{1,0,0\},\{0,1,0\},\left\{1+c_{1}, 1+c_{1}, 2+2 c_{1}\right\}\right\}$ & \\
\hline $8 C 2 / m$ & $32 z$ & $2\left\{\{1,0,0\},\{0,1,0\},\left\{1+c_{1}, 0,2+2 c_{1}\right\}\right\}$ & \\
\hline $8 C 2 / m$ & $32 z$ & $3\left\{\{1,0,0\},\{0,1,0\},\left\{0,1+c_{1}, 2+2 c_{1}\right\}\right\}$ & \\
\hline $8 C 2 / m$ & $32 z$ & $4\left\{\{1,0,0\},\{0,1,0\},\left\{0,0,1+c_{1}\right\}\right\}$ & \\
\hline $8 C 2 / m$ & $32 z$ & $5\left\{\{1,0,0\},\{1,2,0\},\left\{1+c_{1}, 0,2+2 c_{1}\right\}\right\}$ & \\
\hline $8 C 2 / m$ & $32 z$ & $6\left\{\{1,0,0\},\{1,2,0\},\left\{0,0,1+c_{1}\right\}\right\}$ & \\
\hline $8 C 2 / m$ & $32 z$ & $7\left\{\{1,0,0\},\{0,2,0\},\left\{1+c_{1}, 0,2+2 c_{1}\right\}\right\}$ & \\
\hline $8 C 2 / m$ & $32 z$ & $8\left\{\{1,0,0\},\{0,2,0\},\left\{0,0,1+c_{1}\right\}\right\}$ & \\
\hline
\end{tabular}


Table A.1 - continued from previous page

\begin{tabular}{|c|c|c|c|}
\hline ACC & $P_{N}$ & $B_{N}$ matrix row by row & $\mathrm{c \#}$ \\
\hline $8 C 2 / m$ & $32 z$ & $9\left\{\{2,0,0\},\{0,1,0\},\left\{0,1+c_{1}, 2+2 c_{1}\right\}\right\}$ & \\
\hline $8 C 2 / m$ & $32 z$ & $10\left\{\{2,0,0\},\{0,1,0\},\left\{0,0,1+c_{1}\right\}\right\}$ & \\
\hline $8 C 2 / m$ & $32 z$ & $11\left\{\{2,0,0\},\{0,2,0\},\left\{0,0,1+c_{1}\right\}\right\}$ & \\
\hline $8 C 2 / m$ & $4 m_{z}$ & $1\left\{\left\{1+c_{1}, 0,0\right\},\left\{c_{2}, 1+c_{2}+c_{3}, 0\right\},\{0,0,1\}\right\}$ & \\
\hline $8 C 2 / m$ & $4 m_{z}$ & $2\left\{\left\{1+c_{1}, 0,0\right\},\left\{c_{2}, 1+c_{2}+c_{3}, 0\right\},\{0,0,2\}\right\}$ & \\
\hline $8 C 2 / m$ & $4 m_{z}$ & $3\left\{\left\{1+c_{1}, 0,0\right\},\left\{c_{2}, 1+c_{2}+c_{3}, 0\right\},\{0,1,2\}\right\}$ & \\
\hline $8 C 2 / m$ & $4 m_{z}$ & $4\left\{\left\{1+c_{1}, 0,0\right\},\left\{c_{2}, 1+c_{2}+c_{3}, 0\right\},\{1,0,2\}\right\}$ & \\
\hline $8 C 2 / m$ & $4 m_{z}$ & $5\left\{\left\{1+c_{1}, 0,0\right\},\left\{c_{2}, 1+c_{2}+c_{3}, 0\right\},\{1,1,2\}\right\}$ & \\
\hline $8 C 2 / m$ & $52 / m_{z}$ & $1\{\{1,0,0\},\{0,1,0\},\{0,0,1\}\}$ & \\
\hline $8 C 2 / m$ & $52 / m_{z}$ & $2\{\{1,0,0\},\{0,1,0\},\{1,1,2\}\}$ & \\
\hline $8 C 2 / m$ & $52 / m_{z}$ & $3\{\{1,0,0\},\{0,1,0\},\{1,0,2\}\}$ & \\
\hline $8 C 2 / m$ & $52 / m_{z}$ & $4\{\{1,0,0\},\{0,1,0\},\{0,1,2\}\}$ & \\
\hline $8 C 2 / m$ & $52 / m_{z}$ & $5\{\{1,0,0\},\{0,1,0\},\{0,0,2\}\}$ & \\
\hline $8 C 2 / m$ & $52 / m_{z}$ & $6\{\{1,0,0\},\{1,2,0\},\{0,0,1\}\}$ & \\
\hline $8 C 2 / m$ & $52 / m_{z}$ & $7\{\{1,0,0\},\{0,2,0\},\{0,0,1\}\}$ & \\
\hline $8 C 2 / m$ & $52 / m_{z}$ & $8\{\{1,0,0\},\{1,2,0\},\{1,0,2\}\}$ & \\
\hline $8 C 2 / m$ & $52 / m_{z}$ & $9\{\{1,0,0\},\{1,2,0\},\{0,0,2\}\}$ & \\
\hline $8 C 2 / m$ & $52 / m_{z}$ & $10\{\{1,0,0\},\{0,2,0\},\{1,0,2\}\}$ & \\
\hline $8 C 2 / m$ & $52 / m_{z}$ & $11\{\{1,0,0\},\{0,2,0\},\{0,0,2\}\}$ & \\
\hline $8 C 2 / m$ & $52 / m_{z}$ & $12\{\{2,0,0\},\{0,1,0\},\{0,0,1\}\}$ & \\
\hline $8 C 2 / m$ & $52 / m_{z}$ & $13\{\{2,0,0\},\{0,1,0\},\{0,1,2\}\}$ & \\
\hline $8 C 2 / m$ & $52 / m_{z}$ & $14\{\{2,0,0\},\{0,1,0\},\{0,0,2\}\}$ & \\
\hline $8 C 2 / m$ & $52 / m_{z}$ & $15\{\{2,0,0\},\{0,2,0\},\{0,0,1\}\}$ & \\
\hline $8 C 2 / m$ & $52 / m_{z}$ & $16\{\{2,0,0\},\{0,2,0\},\{0,0,2\}\}$ & \\
\hline $9 P 222$ & $22 z$ & $1\left\{\{1,0,0\},\{0,1,0\},\left\{1+c_{1}, 1+c_{1}, 2+2 c_{1}\right\}\right\}$ & \\
\hline $9 P 222$ & $22_{z}$ & $2\left\{\{1,0,0\},\{0,1,0\},\left\{1+c_{1}, 0,2+2 c_{1}\right\}\right\}$ & \\
\hline $9 P 222$ & $22 z$ & $3\left\{\{1,0,0\},\{0,1,0\},\left\{0,1+c_{1}, 2+2 c_{1}\right\}\right\}$ & \\
\hline $9 P 222$ & $22_{z}$ & $4\left\{\{1,0,0\},\{0,1,0\},\left\{0,0,1+c_{1}\right\}\right\}$ & \\
\hline
\end{tabular}


Table A.1 - continued from previous page

\begin{tabular}{|c|c|c|c|}
\hline ACC & $P_{N}$ & $B_{N}$ matrix row by row & $\mathrm{c \#}$ \\
\hline $9 P 222$ & $22_{z}$ & $5\left\{\{1,0,0\},\{1,2,0\},\left\{1+c_{1}, 0,2+2 c_{1}\right\}\right\}$ & \\
\hline $9 P 222$ & $22 z$ & $6\left\{\{1,0,0\},\{1,2,0\},\left\{0,0,1+c_{1}\right\}\right\}$ & \\
\hline $9 P 222$ & $22 z$ & $7\left\{\{1,0,0\},\{0,2,0\},\left\{1+c_{1}, 0,2+2 c_{1}\right\}\right\}$ & \\
\hline $9 P 222$ & $22 z$ & $8\left\{\{1,0,0\},\{0,2,0\},\left\{0,0,1+c_{1}\right\}\right\}$ & \\
\hline $9 P 222$ & $22 z$ & $9\left\{\{2,0,0\},\{0,1,0\},\left\{0,1+c_{1}, 2+2 c_{1}\right\}\right\}$ & \\
\hline $9 P 222$ & $22 z$ & $10\left\{\{2,0,0\},\{0,1,0\},\left\{0,0,1+c_{1}\right\}\right\}$ & \\
\hline $9 P 222$ & $22 z$ & $11\left\{\{2,0,0\},\{0,2,0\},\left\{0,0,1+c_{1}\right\}\right\}$ & \\
\hline $9 P 222$ & $32 y$ & $1\left\{\{1,0,0\},\left\{1+c_{1}, 2+2 c_{1}, 0\right\},\{0,0,1\}\right\}$ & \\
\hline $9 P 222$ & $32 y$ & $2\left\{\{1,0,0\},\left\{0,1+c_{1}, 0\right\},\{0,0,1\}\right\}$ & \\
\hline $9 P 222$ & $32 y$ & $3\left\{\{1,0,0\},\left\{1+c_{1}, 2+2 c_{1}, 0\right\},\{1,0,2\}\right\}$ & \\
\hline $9 P 222$ & $32 y$ & $4\left\{\{1,0,0\},\left\{1+c_{1}, 2+2 c_{1}, 0\right\},\{0,0,2\}\right\}$ & \\
\hline $9 P 222$ & $322_{y}$ & $5\left\{\{1,0,0\},\left\{0,1+c_{1}, 0\right\},\{1,0,2\}\right\}$ & \\
\hline $9 P 222$ & $32 y$ & $6\left\{\{1,0,0\},\left\{0,1+c_{1}, 0\right\},\{1,1,2\}\right\}$ & \\
\hline $9 P 222$ & $32 y$ & $7\left\{\{1,0,0\},\left\{0,1+c_{1}, 0\right\},\{0,0,2\}\right\}$ & \\
\hline $9 P 222$ & $322_{y}$ & $8\left\{\{1,0,0\},\left\{0,1+c_{1}, 0\right\},\{0,1,2\}\right\}$ & \\
\hline $9 P 222$ & $32 y$ & $9\left\{\{2,0,0\},\left\{0,1+c_{1}, 0\right\},\{0,0,1\}\right\}$ & \\
\hline $9 P 222$ & $32 y$ & $10\left\{\{2,0,0\},\left\{0,1+c_{1}, 0\right\},\{0,0,2\}\right\}$ & \\
\hline $9 P 222$ & $322_{y}$ & $11\left\{\{2,0,0\},\left\{0,1+c_{1}, 0\right\},\{0,1,2\}\right\}$ & \\
\hline $9 P 222$ & $42 x$ & $1\left\{\left\{1+c_{1}, 0,0\right\},\{0,1,0\},\{0,0,1\}\right\}$ & \\
\hline $9 P 222$ & $42 x$ & $2\left\{\left\{1+c_{1}, 0,0\right\},\{0,1,0\},\{0,1,2\}\right\}$ & \\
\hline $9 P 222$ & $42 x$ & $3\left\{\left\{1+c_{1}, 0,0\right\},\{0,1,0\},\{1,1,2\}\right\}$ & \\
\hline $9 P 222$ & $42 x$ & $4\left\{\left\{1+c_{1}, 0,0\right\},\{0,1,0\},\{0,0,2\}\right\}$ & \\
\hline $9 P 222$ & $42 x$ & $5\left\{\left\{1+c_{1}, 0,0\right\},\{0,1,0\},\{1,0,2\}\right\}$ & \\
\hline $9 P 222$ & $42 x$ & $6\left\{\left\{1+c_{1}, 0,0\right\},\{0,2,0\},\{0,0,1\}\right\}$ & \\
\hline $9 P 222$ & $42 x$ & $7\left\{\left\{1+c_{1}, 0,0\right\},\{1,2,0\},\{0,0,1\}\right\}$ & \\
\hline $9 P 222$ & $42 x$ & $8\left\{\left\{1+c_{1}, 0,0\right\},\{0,2,0\},\{0,0,2\}\right\}$ & \\
\hline $9 P 222$ & $42 x$ & $9\left\{\left\{1+c_{1}, 0,0\right\},\{0,2,0\},\{1,0,2\}\right\}$ & \\
\hline $9 P 222$ & $42 x$ & $10\left\{\left\{1+c_{1}, 0,0\right\},\{1,2,0\},\{0,0,2\}\right\}$ & \\
\hline
\end{tabular}


Table A.1 - continued from previous page

\begin{tabular}{|c|c|c|c|}
\hline ACC & $P_{N}$ & $B_{N}$ matrix row by row & $\mathrm{c \#}$ \\
\hline $9 P 222$ & $42_{x}$ & $11\left\{\left\{1+c_{1}, 0,0\right\},\{1,2,0\},\{1,0,2\}\right\}$ & \\
\hline $9 P 222$ & 5222 & $1\{\{1,0,0\},\{0,1,0\},\{0,0,1\}\}$ & \\
\hline $9 P 222$ & 5222 & $2\{\{1,0,0\},\{0,1,0\},\{1,1,2\}\}$ & \\
\hline $9 P 222$ & 5222 & $3\{\{1,0,0\},\{0,1,0\},\{1,0,2\}\}$ & \\
\hline $9 P 222$ & 5222 & $4\{\{1,0,0\},\{0,1,0\},\{0,1,2\}\}$ & \\
\hline $9 P 222$ & 5222 & $5\{\{1,0,0\},\{0,1,0\},\{0,0,2\}\}$ & \\
\hline $9 P 222$ & 5222 & $6\{\{1,0,0\},\{1,2,0\},\{0,0,1\}\}$ & \\
\hline $9 P 222$ & 5222 & $7\{\{1,0,0\},\{0,2,0\},\{0,0,1\}\}$ & \\
\hline $9 P 222$ & 5222 & $8\{\{1,0,0\},\{1,2,0\},\{1,0,2\}\}$ & \\
\hline $9 P 222$ & 5222 & $9\{\{1,0,0\},\{1,2,0\},\{0,0,2\}\}$ & \\
\hline $9 P 222$ & 5222 & $10\{\{1,0,0\},\{0,2,0\},\{1,0,2\}\}$ & \\
\hline $9 P 222$ & 5222 & $11\{\{1,0,0\},\{0,2,0\},\{0,0,2\}\}$ & \\
\hline $9 P 222$ & 5222 & $12\{\{2,0,0\},\{0,1,0\},\{0,0,1\}\}$ & \\
\hline $9 P 222$ & 5222 & $13\{\{2,0,0\},\{0,1,0\},\{0,1,2\}\}$ & \\
\hline $9 P 222$ & 5222 & $14\{\{2,0,0\},\{0,1,0\},\{0,0,2\}\}$ & \\
\hline $9 P 222$ & 5222 & $15\{\{2,0,0\},\{0,2,0\},\{0,0,1\}\}$ & \\
\hline $9 P 222$ & 5222 & $16\{\{2,0,0\},\{0,2,0\},\{0,0,2\}\}$ & \\
\hline $10 C 222$ & $22 z$ & $1\left\{\{1,0,0\},\{0,1,0\},\left\{1+c_{1}, 1+c_{1}, 2+2 c_{1}\right\}\right\}$ & \\
\hline $10 C 222$ & $22 z$ & $2\left\{\{1,0,0\},\{0,1,0\},\left\{0,0,1+c_{1}\right\}\right\}$ & \\
\hline $10 C 222$ & $22 z$ & $3\left\{\{1,0,0\},\{1,2,0\},\left\{1+c_{1}, 0,2+2 c_{1}\right\}\right\}$ & \\
\hline $10 C 222$ & $22 z$ & $4\left\{\{1,0,0\},\{1,2,0\},\left\{0,0,1+c_{1}\right\}\right\}$ & \\
\hline $10 C 222$ & $22 z$ & $5\left\{\{2,0,0\},\{0,2,0\},\left\{0,0,1+c_{1}\right\}\right\}$ & \\
\hline $10 C 222$ & $32 y$ & $1\left\{\{1,0,0\},\left\{c_{1}, 1+c_{1}, 0\right\},\{0,0,1\}\right\}$ & \\
\hline $10 C 222$ & $32 y$ & $2\left\{\{1,0,0\},\left\{c_{1}, 1+c_{1}, 0\right\},\{0,0,2\}\right\}$ & \\
\hline $10 C 222$ & $32 y$ & $3\left\{\{1,0,0\},\left\{c_{1}, 1+c_{1}, 0\right\},\{1,1,2\}\right\}$ & \\
\hline $10 C 222$ & $42 x$ & $1\{\{1,0,0\},\{0,1,0\},\{0,0,1\}\}$ & \\
\hline $10 C 222$ & $42 x$ & $2\left\{\{1,0,0\},\left\{1,2+c_{1}, 0\right\},\{0,0,1\}\right\}$ & \\
\hline $10 C 222$ & $42_{x}$ & $3\{\{1,0,0\},\{0,1,0\},\{1,1,2\}\}$ & \\
\hline
\end{tabular}


Table A.1 - continued from previous page

\begin{tabular}{|c|c|c|c|}
\hline ACC & $P_{N}$ & $B_{N}$ matrix row by row & $\mathrm{c \#}$ \\
\hline $10 C 222$ & $42_{x}$ & $4\{\{1,0,0\},\{0,1,0\},\{0,0,2\}\}$ & \\
\hline $10 C 222$ & $42 x$ & $5\left\{\{1,0,0\},\left\{1,2+c_{1}, 0\right\},\{0,0,2\}\right\}$ & \\
\hline $10 C 222$ & $42 x$ & $6\left\{\{1,0,0\},\left\{1,2+c_{1}, 0\right\},\{0,1,2\}\right\}$ & \\
\hline $10 C 222$ & 5222 & $1\{\{1,0,0\},\{1,2,0\},\{0,0,1\}\}$ & \\
\hline $10 C 222$ & 5222 & $2\{\{1,0,0\},\{0,1,0\},\{0,0,1\}\}$ & \\
\hline $10 C 222$ & 5222 & $3\{\{1,0,0\},\{1,2,0\},\{0,0,2\}\}$ & \\
\hline $10 C 222$ & 5222 & $4\{\{1,0,0\},\{0,1,0\},\{1,1,2\}\}$ & \\
\hline $10 C 222$ & 5222 & $5\{\{1,0,0\},\{0,1,0\},\{0,0,2\}\}$ & \\
\hline $11 F 222$ & $22_{z}$ & $1\left\{\{1,0,0\},\left\{c_{1}, 1+c_{1}, 0\right\},\{1,0,2\}\right\}$ & \\
\hline $11 F 222$ & $22 z$ & $2\left\{\{1,0,0\},\left\{c_{1}, 1+c_{1}, 0\right\},\{0,1,2\}\right\}$ & \\
\hline $11 F 222$ & $22 z$ & $3\left\{\{1,0,0\},\left\{c_{1}, 1+c_{1}, 0\right\},\{0,0,1\}\right\}$ & \\
\hline $11 F 222$ & $32 y$ & $1\left\{\{1,0,0\},\{1,2,0\},\left\{c_{1}, 0,1+c_{1}\right\}\right\}$ & \\
\hline $11 F 222$ & $32 y$ & $2\left\{\{1,0,0\},\{0,1,0\},\left\{c_{1}, 1+c_{1}, 2+2 c_{1}\right\}\right\}$ & \\
\hline $11 F 222$ & $32 y$ & $3\left\{\{1,0,0\},\{0,1,0\},\left\{c_{1}, 0,1+c_{1}\right\}\right\}$ & \\
\hline $11 F 222$ & $42 x$ & $1\{\{1,0,0\},\{1,2,0\},\{0,0,1\}\}$ & \\
\hline $11 F 222$ & $42 x$ & $2\left\{\{1,0,0\},\{1,2,0\},\left\{1+c_{1}, c_{1}, 2+c_{1}\right\}\right\}$ & \\
\hline $11 F 222$ & $42 x$ & $3\left\{\{1,0,0\},\{0,1,0\},\left\{0, c_{1}, 1+c_{1}\right\}\right\}$ & \\
\hline $11 F 222$ & $42_{x}$ & $4\left\{\{1,0,0\},\{0,1,0\},\left\{1+c_{1}, c_{1}, 2+2 c_{1}\right\}\right\}$ & \\
\hline $11 F 222$ & 5222 & $1\{\{1,0,0\},\{0,1,0\},\{0,1,2\}\}$ & \\
\hline $11 F 222$ & 5222 & $2\{\{1,0,0\},\{0,1,0\},\{1,0,2\}\}$ & \\
\hline $11 F 222$ & 5222 & $3\{\{1,0,0\},\{0,1,0\},\{0,0,1\}\}$ & \\
\hline $11 F 222$ & 5222 & $4\{\{1,0,0\},\{1,2,0\},\{1,0,2\}\}$ & \\
\hline $11 F 222$ & 5222 & $5\{\{1,0,0\},\{1,2,0\},\{0,0,1\}\}$ & \\
\hline $12 I 222$ & $22 z$ & $1\{\{1,0,0\},\{1,2,0\},\{0,0,2\}\}$ & \\
\hline $12 I 222$ & $22_{z}$ & $2\{\{1,0,0\},\{0,1,0\},\{0,0,1\}\}$ & \\
\hline $12 I 222$ & $22_{z}$ & $3\left\{\{1,0,0\},\{1,2,0\},\left\{2,2,2\left(2+c_{1}\right)\right\}\right\}$ & \\
\hline $12 I 222$ & $22 z$ & $4\left\{\{1,0,0\},\{0,1,0\},\left\{3+2 c_{1}, 3+2 c_{1}, 4\left(1+c_{1}\right)\right\}\right\}$ & \\
\hline $12 I 222$ & $22_{z}$ & $5\left\{\{1,0,0\},\{0,1,0\},\left\{1,1,2+c_{1}\right\}\right\}$ & \\
\hline
\end{tabular}


Table A.1 - continued from previous page

\begin{tabular}{|c|c|c|c|}
\hline ACC & $P_{N}$ & $B_{N}$ matrix row by row & $\mathrm{c \#}$ \\
\hline $12 I 222$ & $32 y$ & $1\{\{1,0,0\},\{0,2,0\},\{1,0,2\}\}$ & \\
\hline $12 I 222$ & $32 y$ & $2\{\{1,0,0\},\{0,1,0\},\{0,0,1\}\}$ & \\
\hline $12 I 222$ & $32 y$ & $3\left\{\{1,0,0\},\{0,2,0\},\left\{3+2 c_{1}, 2,2\left(2+c_{1}\right)\right\}\right\}$ & \\
\hline $12 I 222$ & $32 y$ & $4\left\{\{1,0,0\},\{0,1,0\},\left\{3+4 c_{1}, 3+2 c_{1}, 4\left(1+c_{1}\right)\right\}\right\}$ & \\
\hline $12 I 222$ & $32 y$ & $5\left\{\{1,0,0\},\{0,1,0\},\left\{1+c_{1}, 1,2+c_{1}\right\}\right\}$ & \\
\hline $12 I 222$ & $42 x$ & $1\{\{1,0,0\},\{0,1,0\},\{0,0,1\}\}$ & \\
\hline $12 I 222$ & $42 x$ & $2\left\{\{1,0,0\},\{0,1,0\},\left\{3+2 c_{1}, 3+4 c_{1}, 4\left(1+c_{1}\right)\right\}\right\}$ & \\
\hline $12 I 222$ & $42 x$ & $3\left\{\{1,0,0\},\{0,1,0\},\left\{1,1+c_{1}, 2+c_{1}\right\}\right\}$ & \\
\hline $12 I 222$ & $42_{x}$ & $4\{\{2,0,0\},\{0,1,0\},\{0,1,2\}\}$ & \\
\hline $12 I 222$ & $42_{x}$ & $5\left\{\{2,0,0\},\{0,1,0\},\left\{2,3+2 c_{1}, 2\left(2+c_{1}\right)\right\}\right\}$ & \\
\hline $12 I 222$ & 5222 & $1\{\{1,0,0\},\{0,1,0\},\{3,3,4\}\}$ & \\
\hline $12 I 222$ & 5222 & $2\{\{1,0,0\},\{0,1,0\},\{1,1,2\}\}$ & \\
\hline $12 I 222$ & 5222 & $3\{\{1,0,0\},\{0,1,0\},\{0,0,1\}\}$ & \\
\hline $12 I 222$ & 5222 & $4\{\{1,0,0\},\{0,1,0\},\{3,3,4\}\}$ & \\
\hline $12 I 222$ & 5222 & $5\{\{1,0,0\},\{0,1,0\},\{1,1,2\}\}$ & \\
\hline $12 I 222$ & 5222 & $6\{\{1,0,0\},\{0,1,0\},\{0,0,1\}\}$ & \\
\hline 13 Pmm 2 & $22_{z}$ & $1\left\{\{1,0,0\},\{0,1,0\},\left\{1+c_{1}, 1+c_{1}, 2+2 c_{1}\right\}\right\}$ & \\
\hline 13 Pmm 2 & $22 z$ & $2\left\{\{1,0,0\},\{0,1,0\},\left\{1+c_{1}, 0,2+2 c_{1}\right\}\right\}$ & \\
\hline $13 P m m 2$ & $22 z$ & $3\left\{\{1,0,0\},\{0,1,0\},\left\{0,1+c_{1}, 2+2 c_{1}\right\}\right\}$ & \\
\hline 13 Pmm 2 & $22 z$ & $4\left\{\{1,0,0\},\{0,1,0\},\left\{0,0,1+c_{1}\right\}\right\}$ & \\
\hline 13 Pmm 2 & $22 z$ & $5\left\{\{1,0,0\},\{1,2,0\},\left\{1+c_{1}, 0,2+2 c_{1}\right\}\right\}$ & \\
\hline 13 Pmm 2 & $22 z$ & $6\left\{\{1,0,0\},\{1,2,0\},\left\{0,0,1+c_{1}\right\}\right\}$ & \\
\hline 13 Pmm 2 & $22 z$ & $7\left\{\{1,0,0\},\{0,2,0\},\left\{1+c_{1}, 0,2+2 c_{1}\right\}\right\}$ & \\
\hline 13 Pmm2 & $22 z$ & $8\left\{\{1,0,0\},\{0,2,0\},\left\{0,0,1+c_{1}\right\}\right\}$ & \\
\hline 13 Pmm 2 & $22_{z}$ & $9\left\{\{2,0,0\},\{0,1,0\},\left\{0,1+c_{1}, 2+2 c_{1}\right\}\right\}$ & \\
\hline 13 Pmm 2 & $22_{z}$ & $10\left\{\{2,0,0\},\{0,1,0\},\left\{0,0,1+c_{1}\right\}\right\}$ & \\
\hline 13 Pmm 2 & $22 z$ & $11\left\{\{2,0,0\},\{0,2,0\},\left\{0,0,1+c_{1}\right\}\right\}$ & \\
\hline 13 Pmm2 & $3 m_{x}$ & $1\left\{\{1,0,0\},\left\{1+c_{1}, 2\left(1+c_{1}\right), 0\right\},\left\{1+c_{2}, 0,2\left(1+c_{2}\right)\right\}\right\}$ & \\
\hline
\end{tabular}


Table A.1 - continued from previous page

\begin{tabular}{|c|c|c|c|}
\hline ACC & $P_{N}$ & $B_{N}$ matrix row by row & $\mathrm{c} \#$ \\
\hline 13 Pmm 2 & $3 m_{x}$ & $2\left\{\{1,0,0\},\left\{1+c_{1}, 2\left(1+c_{1}\right), 0\right\},\left\{0,0,1+c_{2}\right\}\right\}$ & \\
\hline 13 Pmm2 & $3 m_{x}$ & $3\left\{\{1,0,0\},\left\{0,1+c_{1}, 0\right\},\left\{0,0,1+c_{2}\right\}\right\}$ & \\
\hline $13 \mathrm{Pmm} 2$ & $3 m_{x}$ & $4\left\{\{1,0,0\},\left\{0,1+c_{1}, 0\right\},\left\{0,1+c_{2}, 2\left(1+c_{2}\right)\right\}\right\}$ & \\
\hline 13 Pmm2 & $3 m_{x}$ & $5\left\{\{2,0,0\},\left\{0,1+c_{1}, 0\right\},\left\{0,0,1+c_{2}\right\}\right\}$ & \\
\hline 13 Pmm2 & $3 m_{x}$ & $6\left\{\{2,0,0\},\left\{0,1+c_{1}, 0\right\},\left\{0,1+c_{2}, 2\left(1+c_{2}\right)\right\}\right\}$ & \\
\hline 13 Pmm2 & $3 m_{x}$ & $7\left\{\{1,0,0\},\left\{0,1+c_{1}, 0\right\},\left\{1+c_{2}, 0,2\left(1+c_{2}\right)\right\}\right\}$ & \\
\hline 13 Pmm 2 & $3 m_{x}$ & $8\left\{\{1,0,0\},\left\{0,1+c_{1}, 0\right\},\left\{2\left(1+c_{2}\right), 2\left(1+c_{2}\right), 4\left(1+c_{2}\right)\right\}\right\}$ & \\
\hline 13 Pmm2 & $3 m_{x}$ & $9\left\{\{1,0,0\},\left\{0,1+c_{1}, 0\right\},\left\{3+2 c_{2}, 3+2 c_{2}, 6+4 c_{2}\right\}\right\}$ & \\
\hline 13 Pmm 2 & $3 m_{x}$ & $10\left\{\{1,0,0\},\left\{0,1+c_{1}, 0\right\},\left\{1+2 c_{2}, 1+2 c_{2}, 2+4 c_{2}\right\}\right\}$ & \\
\hline 13 Pmm2 & $3 m_{x}$ & $11\left\{\{1,0,0\},\left\{0,1+c_{1}, 0\right\},\left\{2\left(1+c_{2}\right), 2\left(1+c_{2}\right), 4\left(1+c_{2}\right)\right\}\right\}$ & \\
\hline 13 Pmm2 & $4 m_{y}$ & $1\left\{\left\{1+c_{1}, 0,0\right\},\{0,1,0\},\left\{0,1+c_{2}, 2\left(1+c_{2}\right)\right\}\right\}$ & \\
\hline 13 Pmm2 & $4 m_{y}$ & $2\left\{\left\{1+c_{1}, 0,0\right\},\{0,1,0\},\left\{2\left(1+c_{2}\right), 2\left(1+c_{2}\right), 4\left(1+c_{2}\right)\right\}\right\}$ & \\
\hline 13 Pmm2 & $4 m_{y}$ & $3\left\{\left\{1+c_{1}, 0,0\right\},\{0,1,0\},\left\{1+2 c_{2}, 1+2 c_{2}, 2+4 c_{2}\right\}\right\}$ & \\
\hline $13 \operatorname{Pmm} 2$ & $4 m_{y}$ & $4\left\{\left\{1+c_{1}, 0,0\right\},\{0,1,0\},\left\{0,0,2\left(1+c_{2}\right)\right\}\right\}$ & \\
\hline $13 \operatorname{Pmm} 2$ & $4 m_{y}$ & $5\left\{\left\{1+c_{1}, 0,0\right\},\{0,1,0\},\left\{2\left(1+c_{2}\right), 0,4\left(1+c_{2}\right)\right\}\right\}$ & \\
\hline $13 P m m 2$ & $4 m_{y}$ & $6\left\{\left\{1+c_{1}, 0,0\right\},\{0,1,0\},\left\{0,0,1+2 c_{2}\right\}\right\}$ & \\
\hline 13 Pmm 2 & $4 m_{y}$ & $7\left\{\left\{1+c_{1}, 0,0\right\},\{0,1,0\},\left\{1+2 c_{2}, 0,2+4 c_{2}\right\}\right\}$ & \\
\hline 13 Pmm 2 & $4 m_{y}$ & $8\left\{\left\{1+c_{1}, 0,0\right\},\{0,2,0\},\left\{0,0,1+c_{2}\right\}\right\}$ & \\
\hline $13 \operatorname{Pmm} 2$ & $4 m_{y}$ & $9\left\{\left\{1+c_{1}, 0,0\right\},\{0,2,0\},\left\{1+c_{2}, 0,2\left(1+c_{2}\right)\right\}\right\}$ & \\
\hline 13 Pmm 2 & $4 m_{y}$ & $10\left\{\left\{1+c_{1}, 0,0\right\},\{1,2,0\},\left\{0,0,1+c_{2}\right\}\right\}$ & \\
\hline $13 \operatorname{Pmm} 2$ & $4 m_{y}$ & $11\left\{\left\{1+c_{1}, 0,0\right\},\{1,2,0\},\left\{1+c_{2}, 0,2\left(1+c_{2}\right)\right\}\right\}$ & \\
\hline $13 \mathrm{Pmm} 2$ & $5 m m 2_{z}$ & $1\left\{\{1,0,0\},\{0,1,0\},\left\{1+c_{1}, 1+c_{1}, 2+2 c_{1}\right\}\right\}$ & \\
\hline 13 Pmm2 & $5 m m 2_{z}$ & $2\left\{\{1,0,0\},\{0,1,0\},\left\{1+c_{1}, 0,2+2 c_{1}\right\}\right\}$ & \\
\hline 13 Pmm2 & $5 m m 2_{z}$ & $3\left\{\{1,0,0\},\{0,1,0\},\left\{0,1+c_{1}, 2+2 c_{1}\right\}\right\}$ & \\
\hline 13 Pmm2 & $5 m m 2_{z}$ & $4\left\{\{1,0,0\},\{0,1,0\},\left\{0,0,1+c_{1}\right\}\right\}$ & \\
\hline 13 Pmm2 & $5 m m 2_{z}$ & $5\left\{\{1,0,0\},\{1,2,0\},\left\{1+c_{1}, 0,2+2 c_{1}\right\}\right\}$ & \\
\hline 13 Pmm2 & $5 m m 2_{z}$ & $6\left\{\{1,0,0\},\{1,2,0\},\left\{0,0,1+c_{1}\right\}\right\}$ & \\
\hline 13 Pmm2 & $5 m m 2_{z}$ & $7\left\{\{1,0,0\},\{0,2,0\},\left\{1+c_{1}, 0,2+2 c_{1}\right\}\right\}$ & \\
\hline
\end{tabular}


Table A.1 - continued from previous page

\begin{tabular}{|c|c|c|c|}
\hline ACC & $P_{N}$ & $B_{N}$ matrix row by row & $\mathrm{c} \#$ \\
\hline 13 Pmm 2 & $5 m m 2_{z}$ & $8\left\{\{1,0,0\},\{0,2,0\},\left\{0,0,1+c_{1}\right\}\right\}$ & \\
\hline 13 Pmm 2 & $5 m m 2_{z}$ & $9\left\{\{2,0,0\},\{0,1,0\},\left\{0,1+c_{1}, 2+2 c_{1}\right\}\right\}$ & \\
\hline 13 Pmm 2 & $5 m m 2_{z}$ & $10\left\{\{2,0,0\},\{0,1,0\},\left\{0,0,1+c_{1}\right\}\right\}$ & \\
\hline 13 Pmm2 & $5 m m 2_{z}$ & $11\left\{\{2,0,0\},\{0,2,0\},\left\{0,0,1+c_{1}\right\}\right\}$ & \\
\hline $14 \mathrm{Cmm} 2$ & $22 z$ & $1\left\{\{1,0,0\},\{0,1,0\},\left\{1+c_{1}, 1+c_{1}, 2+2 c_{1}\right\}\right\}$ & \\
\hline $14 \mathrm{Cmm} 2$ & $22_{z}$ & $2\left\{\{1,0,0\},\{0,1,0\},\left\{0,0,1+c_{1}\right\}\right\}$ & \\
\hline $14 \mathrm{Cmm} 2$ & $22_{z}$ & $3\left\{\{1,0,0\},\{1,2,0\},\left\{1+c_{1}, 0,2+2 c_{1}\right\}\right\}$ & \\
\hline $14 \mathrm{Cmm} 2$ & $22_{z}$ & $4\left\{\{1,0,0\},\{1,2,0\},\left\{0,0,1+c_{1}\right\}\right\}$ & \\
\hline $14 \mathrm{Cmm} 2$ & $22_{z}$ & $5\left\{\{2,0,0\},\{0,2,0\},\left\{0,0,1+c_{1}\right\}\right\}$ & \\
\hline $14 \mathrm{Cmm} 2$ & $3 m_{x}$ & $1\left\{\{1,0,0\},\left\{c_{1}, 1+c_{1}, 0\right\},\left\{0,0,1+c_{2}\right\}\right\}$ & \\
\hline $14 \mathrm{Cmm} 2$ & $3 m_{x}$ & $2\left\{\{1,0,0\},\left\{c_{1}, 1+c_{1}, 0\right\},\left\{1+c_{2}, 1+c_{2}, 2\left(1+c_{2}\right)\right\}\right\}$ & \\
\hline $14 \mathrm{Cmm} 2$ & $4 m_{y}$ & $1\left\{\{1,0,0\},\{0,1,0\},\left\{0,0,1+c_{1}\right\}\right\}$ & \\
\hline $14 \mathrm{Cmm} 2$ & $4 m_{y}$ & $2\left\{\{1,0,0\},\{0,1,0\},\left\{1+c_{1}, 1+c_{1}, 2\left(1+c_{1}\right)\right\}\right\}$ & \\
\hline $14 \mathrm{Cmm} 2$ & $4 m_{y}$ & $3\left\{\{1,0,0\},\left\{1,2+c_{1}, 0\right\},\left\{0,0,1+c_{2}\right\}\right\}$ & \\
\hline $14 \mathrm{Cmm} 2$ & $4 m_{y}$ & $4\left\{\{1,0,0\},\left\{1,2+c_{1}, 0\right\},\left\{0,1+c_{2}, 2\left(1+c_{2}\right)\right\}\right\}$ & \\
\hline $14 C m m 2$ & $5 m m 2_{z}$ & $1\left\{\{1,0,0\},\{1,2,0\},\left\{0,0,1+c_{1}\right\}\right\}$ & \\
\hline $14 \mathrm{Cmm} 2$ & $5 m m 2_{z}$ & $2\left\{\{1,0,0\},\{0,1,0\},\left\{1+c_{1}, 1+c_{1}, 2+2 c_{1}\right\}\right\}$ & \\
\hline $14 \mathrm{Cmm} 2$ & $5 m m 2_{z}$ & $3\left\{\{1,0,0\},\{0,1,0\},\left\{0,0,1+c_{1}\right\}\right\}$ & \\
\hline $15 \mathrm{Amm} 2$ & $22 z$ & $1\left\{\{1,0,0\},\{0,1,0\},\left\{1+c_{1}, 1+2 c_{1}, 2+2 c_{1}\right\}\right\}$ & \\
\hline 15 Amm 2 & $22 z$ & $2\left\{\{1,0,0\},\{0,1,0\},\left\{0, c_{1}, 1+c_{1}\right\}\right\}$ & \\
\hline 15 Amm 2 & $22 z$ & $3\left\{\{2,0,0\},\{0,1,0\},\left\{0, c_{1}, 1+c_{1}\right\}\right\}$ & \\
\hline 15 Amm 2 & $3 m_{x}$ & $1\{\{1,0,0\},\{1,2,0\},\{1,0,2\}\}$ & \\
\hline $15 \mathrm{Amm} 2$ & $3 m_{x}$ & $2\left\{\{1,0,0\},\{1,2,0\},\left\{3+2 c_{1}, 2\left(1+c_{1}\right), 2\left(2+c_{1}\right)\right\}\right\}$ & \\
\hline 15 Amm 2 & $3 m_{x}$ & $3\left\{\{1,0,0\},\{1,2,0\},\left\{5+2 c_{1}, 2,4\left(2+c_{1}\right)\right\}\right\}$ & \\
\hline 15 Amm 2 & $3 m_{x}$ & $4\left\{\{1,0,0\},\left\{2+c_{1}, 2\left(2+c_{1}\right), 0\right\},\left\{2+c_{1}, 0,2\left(2+c_{1}\right)\right\}\right\}$ & \\
\hline 15 Amm2 & $3 m_{x}$ & $5\left\{\{1,0,0\},\left\{2+c_{1}, 2\left(2+c_{1}\right), 0\right\}\right.$ & \\
\hline & & $\left.\left\{\left(2+c_{1}\right)\left(3+2 c_{2}\right), 2\left(2+c_{1}\right)\left(1+c_{2}\right), 2\left(2+c_{1}\right)\left(2+c_{2}\right)\right\}\right\}$ & \\
\hline 15 Amm 2 & $3 m_{x}$ & $6\left\{\{1,0,0\},\{1,2,0\},\left\{7+2 d_{1}+2 d_{2}, 2\left(2+d_{2}\right), 2\left(5+2 d_{1}+d_{2}\right)\right\}\right\}$ & 21 \\
\hline
\end{tabular}


Table A.1 - continued from previous page

\begin{tabular}{|c|c|c|c|}
\hline ACC & $P_{N}$ & $B_{N}$ matrix row by row & $\mathrm{c \#}$ \\
\hline $15 \mathrm{Amm} 2$ & $3 m_{x}$ & $\begin{aligned} 7 & \left\{\{1,0,0\},\left\{2+c_{1}, 2\left(2+c_{1}\right), 0\right\}\right. \\
& \left.\left\{\left(2+c_{1}\right)\left(5+2 d_{1}+2 d_{2}\right), 2\left(2+c_{1}\right)\left(1+d_{2}\right), 2\left(2+c_{1}\right)\left(4+2 d_{1}+d_{2}\right)\right\}\right\}\end{aligned}$ & 57 \\
\hline 15 Amm2 & $3 m_{x}$ & $8\{\{1,0,0\},\{2,4,0\},\{2,4,8\}\}$ & \\
\hline 15 Amm 2 & $3 m_{x}$ & $9\left\{\{1,0,0\},\{1,2,0\},\left\{1,2,2\left(2+c_{1}\right)\right\}\right\}$ & \\
\hline 15 Amm 2 & $3 m_{x}$ & $10\left\{\{1,0,0\},\left\{3+c_{1}, 2\left(3+c_{1}\right), 0\right\},\left\{3+c_{1}, 2\left(3+c_{1}\right), 4\left(3+c_{1}\right)\right\}\right\}$ & \\
\hline 15 Amm 2 & $3 m_{x}$ & $11\left\{\{1,0,0\},\{1,2,0\},\left\{3+2 d_{1}, 6+4 d_{1}, 2\left(4+2 d_{1}+d_{2}\right)\right\}\right\}$ & 20 \\
\hline 15 Amm 2 & $3 m_{x}$ & $12\left\{\{1,0,0\},\{2,4,0\},\left\{2+4 d_{1}, 4+8 d_{1}, 4\left(3+2 d_{1}+d_{2}\right)\right\}\right\}$ & 19 \\
\hline $15 \mathrm{Amm} 2$ & $3 m_{x}$ & $13\left\{\{1,0,0\},\{2,4,0\},\left\{6+4 d_{1}, 12+8 d_{1}, 4\left(4+2 d_{1}+d_{2}\right)\right\}\right\}$ & 20 \\
\hline $15 \mathrm{Amm} 2$ & $3 m_{x}$ & $\begin{aligned} 14 & \left\{\{1,0,0\},\left\{3+c_{1}, 2\left(3+c_{1}\right), 0\right\}\right. \\
& \left.\left\{\left(3+c_{1}\right)\left(1+2 d_{1}\right),\left(3+c_{1}\right)\left(2+4 d_{1}\right), 2\left(3+c_{1}\right)\left(3+2 d_{1}+d_{2}\right)\right\}\right\}\end{aligned}$ & 54 \\
\hline 15 Amm 2 & $3 m_{x}$ & $\begin{aligned} 15 & \left\{\{1,0,0\},\left\{3+c_{1}, 2\left(3+c_{1}\right), 0\right\}\right. \\
& \left.\left\{\left(3+c_{1}\right)\left(3+2 d_{1}\right), 2\left(3+c_{1}\right)\left(3+2 d_{1}\right), 2\left(3+c_{1}\right)\left(4+2 d_{1}+d_{2}\right)\right\}\right\}\end{aligned}$ & 56 \\
\hline 15 Amm2 & $3 m_{x}$ & $16\{\{1,0,0\},\{0,1,0\},\{0,0,1\}\}$ & \\
\hline 15 Amm2 & $3 m_{x}$ & $17\{\{1,0,0\},\{0,2,0\},\{0,2,6\}\}$ & \\
\hline 15 Amm 2 & $3 m_{x}$ & $18\left\{\{1,0,0\},\{0,1,0\},\left\{0,1,3+c_{1}\right\}\right\}$ & \\
\hline 15 Amm 2 & $3 m_{x}$ & $19\left\{\{1,0,0\},\{0,1,0\},\left\{0,1+c_{1}, 2+c_{1}\right\}\right\}$ & \\
\hline 15 Amm 2 & $3 m_{x}$ & $20\left\{\{1,0,0\},\left\{0,2+c_{1}, 0\right\},\left\{0,0,2+c_{1}\right\}\right\}$ & \\
\hline 15 Amm 2 & $3 m_{x}$ & $21\left\{\{1,0,0\},\left\{0,3+c_{1}, 0\right\},\left\{0,3+c_{1}, 3\left(3+c_{1}\right)\right\}\right\}$ & \\
\hline $15 \mathrm{Amm} 2$ & $3 m_{x}$ & $22\left\{\{1,0,0\},\left\{0,2+c_{1}, 0\right\},\left\{0,\left(2+c_{1}\right)\left(1+c_{2}\right),\left(2+c_{1}\right)\left(2+c_{2}\right)\right\}\right\}$ & \\
\hline $15 \mathrm{Amm} 2$ & $3 m_{x}$ & $23\left\{\{1,0,0\},\{0,1,0\},\left\{0,2+d_{1}, 4+d_{1}+d_{2}\right\}\right\}$ & 14 \\
\hline $15 \mathrm{Amm} 2$ & $3 m_{x}$ & $24\left\{\{1,0,0\},\{0,2,0\},\left\{0,2\left(1+d_{1}\right), 2\left(4+d_{1}+d_{2}\right)\right\}\right\}$ & 12 \\
\hline 15 Amm 2 & $3 m_{x}$ & $25\left\{\{1,0,0\},\{0,2,0\},\left\{0,2\left(2+d_{1}\right), 2\left(4+d_{1}+d_{2}\right)\right\}\right\}$ & 14 \\
\hline 15 Amm 2 & $3 m_{x}$ & $26\left\{\{1,0,0\},\left\{0,3+c_{1}, 0\right\},\left\{0,\left(3+c_{1}\right)\left(1+d_{1}\right),\left(3+c_{1}\right)\left(4+d_{1}+d_{2}\right)\right\}\right\}$ & 45 \\
\hline 15 Amm2 & $3 m_{x}$ & $27\left\{\{1,0,0\},\left\{0,3+c_{1}, 0\right\},\left\{0,\left(3+c_{1}\right)\left(2+d_{1}\right),\left(3+c_{1}\right)\left(4+d_{1}+d_{2}\right)\right\}\right\}$ & 47 \\
\hline 15 Amm 2 & $3 m_{x}$ & $28\{\{2,0,0\},\{0,1,0\},\{0,0,1\}\}$ & \\
\hline 15 Amm 2 & $3 m_{x}$ & $29\{\{2,0,0\},\{0,2,0\},\{0,2,6\}\}$ & \\
\hline 15 Amm 2 & $3 m_{x}$ & $30\left\{\{2,0,0\},\{0,1,0\},\left\{0,1,3+c_{1}\right\}\right\}$ & \\
\hline 15 Amm 2 & $3 m_{x}$ & $31\left\{\{2,0,0\},\{0,1,0\},\left\{0,1+c_{1}, 2+c_{1}\right\}\right\}$ & \\
\hline
\end{tabular}


Table A.1 - continued from previous page

\begin{tabular}{|c|c|c|c|}
\hline ACC & $P_{N}$ & $B_{N}$ matrix row by row & $\mathrm{c} \#$ \\
\hline 15 Amm 2 & $3 m_{x}$ & $32\left\{\{2,0,0\},\left\{0,2+c_{1}, 0\right\},\left\{0,0,2+c_{1}\right\}\right\}$ & \\
\hline $15 \mathrm{Amm} 2$ & $3 m_{x}$ & $33\left\{\{2,0,0\},\left\{0,3+c_{1}, 0\right\},\left\{0,3+c_{1}, 3\left(3+c_{1}\right)\right\}\right\}$ & \\
\hline $15 \mathrm{Amm} 2$ & $3 m_{x}$ & $34\left\{\{2,0,0\},\left\{0,2+c_{1}, 0\right\},\left\{0,\left(2+c_{1}\right)\left(1+c_{2}\right),\left(2+c_{1}\right)\left(2+c_{2}\right)\right\}\right\}$ & \\
\hline $15 \mathrm{Amm} 2$ & $3 m_{x}$ & $35\left\{\{2,0,0\},\{0,1,0\},\left\{0,2+d_{1}, 4+d_{1}+d_{2}\right\}\right\}$ & 14 \\
\hline 15 Amm2 & $3 m_{x}$ & $36\left\{\{2,0,0\},\{0,2,0\},\left\{0,2\left(1+d_{1}\right), 2\left(4+d_{1}+d_{2}\right)\right\}\right\}$ & 12 \\
\hline 15 Amm2 & $3 m_{x}$ & $37\left\{\{2,0,0\},\{0,2,0\},\left\{0,2\left(2+d_{1}\right), 2\left(4+d_{1}+d_{2}\right)\right\}\right\}$ & 14 \\
\hline 15 Amm2 & $3 m_{x}$ & $38\left\{\{2,0,0\},\left\{0,3+c_{1}, 0\right\},\left\{0,\left(3+c_{1}\right)\left(1+d_{1}\right),\left(3+c_{1}\right)\left(4+d_{1}+d_{2}\right)\right\}\right\}$ & 45 \\
\hline 15 Amm2 & $3 m_{x}$ & $39\left\{\{2,0,0\},\left\{0,3+c_{1}, 0\right\},\left\{0,\left(3+c_{1}\right)\left(2+d_{1}\right),\left(3+c_{1}\right)\left(4+d_{1}+d_{2}\right)\right\}\right\}$ & 47 \\
\hline 15 Amm2 & $3 m_{x}$ & $40\{\{1,0,0\},\{0,2,0\},\{2,2,4\}\}$ & \\
\hline 15 Amm 2 & $3 m_{x}$ & $41\{\{1,0,0\},\{0,2,0\},\{4,2,8\}\}$ & \\
\hline 15 Amm2 & $3 m_{x}$ & $42\left\{\{1,0,0\},\left\{0,2\left(1+c_{1}\right), 0\right\},\left\{4\left(1+c_{1}\right), 6\left(1+c_{1}\right), 8\left(1+c_{1}\right)\right\}\right\}$ & \\
\hline 15 Amm 2 & $3 m_{x}$ & $43\left\{\{1,0,0\},\left\{0,2\left(2+c_{1}\right), 0\right\},\left\{2\left(2+c_{1}\right), 2\left(2+c_{1}\right), 4\left(2+c_{1}\right)\right\}\right\}$ & \\
\hline 15 Amm 2 & $3 m_{x}$ & $44\left\{\{1,0,0\},\left\{0,2\left(2+c_{1}\right), 0\right\},\left\{4\left(2+c_{1}\right), 2\left(2+c_{1}\right), 8\left(2+c_{1}\right)\right\}\right\}$ & \\
\hline 15 Amm2 & $3 m_{x}$ & $\begin{aligned} 45\left\{\{1,0,0\},\left\{0,2\left(1+c_{1}\right), 0\right\},\right. \\
\\
\left.\left\{2\left(1+c_{1}\right)\left(3+c_{2}\right), 2\left(1+c_{1}\right)\left(5+2 c_{2}\right), 4\left(1+c_{1}\right)\left(3+c_{2}\right)\right\}\right\}\end{aligned}$ & \\
\hline $15 \mathrm{Amm} 2$ & $3 m_{x}$ & $46\left\{\{1,0,0\},\{0,2,0\},\left\{2\left(4+d_{1}+2 d_{2}\right), 6+4 d_{1}+4 d_{2}, 4\left(4+d_{1}+2 d_{2}\right)\right\}\right\}$ & 26 \\
\hline $15 \mathrm{Amm} 2$ & $3 m_{x}$ & $47\left\{\{1,0,0\},\{0,2,0\},\left\{2\left(4+d_{1}+2 d_{2}\right), 10+4 d_{1}+4 d_{2}, 4\left(4+d_{1}+2 d_{2}\right)\right\}\right\}$ & 27 \\
\hline $15 \mathrm{Amm} 2$ & $3 m_{x}$ & $48\left\{\{1,0,0\},\{0,2,0\},\left\{2\left(3+d_{1}+2 d_{2}\right), 2+4 d_{1}+4 d_{2}, 4\left(3+d_{1}+2 d_{2}\right)\right\}\right\}$ & 29 \\
\hline $15 \mathrm{Amm} 2$ & $3 m_{x}$ & $49\left\{\{1,0,0\},\{0,2,0\},\left\{2\left(3+d_{1}+2 d_{2}\right), 6+4 d_{1}+4 d_{2}, 4\left(3+d_{1}+2 d_{2}\right)\right\}\right\}$ & 28 \\
\hline 15 Amm 2 & $3 m_{x}$ & $\begin{aligned} 50 & \left\{\{1,0,0\},\left\{0,2\left(2+c_{1}\right), 0\right\}\right. \\
& \left.\left\{2\left(2+c_{1}\right)\left(3+d_{1}+d_{2}\right), 2\left(2+c_{1}\right)\left(1+2 d_{1}\right), 4\left(2+c_{1}\right)\left(3+d_{1}+d_{2}\right)\right\}\right\}\end{aligned}$ & 37 \\
\hline 15 Amm2 & $3 m_{x}$ & $\begin{aligned} 51 & \left\{\{1,0,0\},\left\{0,2\left(2+c_{1}\right), 0\right\}\right. \\
& \left.\left\{2\left(2+c_{1}\right)\left(3+d_{1}+d_{2}\right), 2\left(2+c_{1}\right)\left(3+2 d_{1}\right), 4\left(2+c_{1}\right)\left(3+d_{1}+d_{2}\right)\right\}\right\}\end{aligned}$ & 40 \\
\hline $15 \mathrm{Amm} 2$ & $3 m_{x}$ & $52\left\{\{1,0,0\},\left\{0,2\left(2+c_{1}\right), 0\right\},\left\{4\left(2+c_{1}\right), 6\left(2+c_{1}\right), 8\left(2+c_{1}\right)\right\}\right\}$ & \\
\hline 15 Amm2 & $3 m_{x}$ & $53\left\{\{1,0,0\},\left\{0,1+2 c_{1}, 0\right\},\left\{2+4 c_{1}, 1+2 c_{1}, 4+8 c_{1}\right\}\right\}$ & \\
\hline 15 Amm2 & $3 m_{x}$ & $54\left\{\{1,0,0\},\left\{0,3+2 c_{1}, 0\right\},\left\{3+2 c_{1}, 3+2 c_{1}, 6+4 c_{1}\right\}\right\}$ & \\
\hline 15 Amm2 & $3 m_{x}$ & $55\left\{\{1,0,0\},\left\{0,3+2 c_{1}, 0\right\},\left\{9+6 c_{1}, 5\left(3+2 c_{1}\right), 6\left(3+2 c_{1}\right)\right\}\right\}$ & \\
\hline 15 Amm2 & $3 m_{x}$ & $56\left\{\{1,0,0\},\left\{0,1+2 c_{1}, 0\right\},\left\{\left(1+2 c_{1}\right)\left(3+c_{2}\right), 1+2 c_{1}, 2\left(1+2 c_{1}\right)\left(3+c_{2}\right)\right\}\right\}$ & \\
\hline
\end{tabular}


Table A.1 - continued from previous page

\begin{tabular}{|c|c|c|c|}
\hline ACC & $P_{N}$ & $B_{N}$ matrix row by row & $\mathrm{c} \#$ \\
\hline 15 Amm2 & $3 m_{x}$ & $\begin{aligned} 57 & \left\{\{1,0,0\},\left\{0,3+2 c_{1}, 0\right\}\right. \\
& \left.\left\{\left(3+2 c_{1}\right)\left(4+c_{2}\right),\left(3+2 c_{1}\right)\left(7+2 c_{2}\right), 2\left(3+2 c_{1}\right)\left(4+c_{2}\right)\right\}\right\}\end{aligned}$ & \\
\hline 15 Amm 2 & $3 m_{x}$ & $58\left\{\{1,0,0\},\{0,1,0\},\left\{4+d_{1}+d_{2}, 3+2 d_{1}, 2\left(4+d_{1}+d_{2}\right)\right\}\right\}$ & 13 \\
\hline $15 \mathrm{Amm} 2$ & $3 m_{x}$ & $59\left\{\{1,0,0\},\{0,1,0\},\left\{4+d_{1}+d_{2}, 5+2 d_{1}, 2\left(4+d_{1}+d_{2}\right)\right\}\right\}$ & 15 \\
\hline $15 \mathrm{Amm} 2$ & $3 m_{x}$ & $\begin{aligned} 60\left\{\{1,0,0\},\left\{0,2\left(1+c_{1}\right), 0\right\}\right. \\
\\
\left.\left\{2\left(1+c_{1}\right)\left(4+d_{1}+d_{2}\right), 2\left(1+c_{1}\right)\left(3+2 d_{1}\right), 4\left(1+c_{1}\right)\left(4+d_{1}+d_{2}\right)\right\}\right\}\end{aligned}$ & 46 \\
\hline 15 Amm2 & $3 m_{x}$ & $\begin{aligned} 61 & \left\{\{1,0,0\},\left\{0,2\left(1+c_{1}\right), 0\right\}\right. \\
& \left.\left\{2\left(1+c_{1}\right)\left(4+d_{1}+d_{2}\right), 2\left(1+c_{1}\right)\left(5+2 d_{1}\right), 4\left(1+c_{1}\right)\left(4+d_{1}+d_{2}\right)\right\}\right\}\end{aligned}$ & 48 \\
\hline 15 Amm 2 & $3 m_{x}$ & $62\{\{1,0,0\},\{0,1,0\},\{2,3,4\}\}$ & \\
\hline 15 Amm 2 & $3 m_{x}$ & $63\left\{\{1,0,0\},\left\{0,2\left(1+c_{1}\right), 0\right\},\left\{4\left(1+c_{1}\right), 6\left(1+c_{1}\right), 8\left(1+c_{1}\right)\right\}\right\}$ & \\
\hline $15 \mathrm{Amm} 2$ & $3 m_{x}$ & $64\left\{\{1,0,0\},\left\{0,1+2 c_{1}, 0\right\},\left\{1+2 c_{1}, 1+2 c_{1}, 2+4 c_{1}\right\}\right\}$ & \\
\hline $15 A m m 2$ & $3 m_{x}$ & $65\left\{\{1,0,0\},\left\{0,1+2 c_{1}, 0\right\},\left\{\left(1+2 c_{1}\right)\left(2+c_{2}\right), 1+2 c_{1}, 2\left(1+2 c_{1}\right)\left(2+c_{2}\right)\right\}\right\}$ & \\
\hline $15 A m m 2$ & $3 m_{x}$ & $66\left\{\{1,0,0\},\{0,1,0\},\left\{3+d_{1}+d_{2}, 3+2 d_{1}, 2\left(3+d_{1}+d_{2}\right)\right\}\right\}$ & 8 \\
\hline $15 A m m 2$ & $3 m_{x}$ & $67\left\{\{1,0,0\},\{0,1,0\},\left\{3+d_{1}+d_{2}, 5+2 d_{1}, 2\left(3+d_{1}+d_{2}\right)\right\}\right\}$ & 11 \\
\hline 15 Amm 2 & $3 m_{x}$ & $\begin{aligned} 68 & \left\{\{1,0,0\},\left\{0,2\left(1+c_{1}\right), 0\right\}\right. \\
& \left.\left\{2\left(1+c_{1}\right)\left(3+d_{1}+d_{2}\right), 2\left(1+c_{1}\right)\left(3+2 d_{1}\right), 4\left(1+c_{1}\right)\left(3+d_{1}+d_{2}\right)\right\}\right\}\end{aligned}$ & 41 \\
\hline $15 \mathrm{Amm} 2$ & $3 m_{x}$ & $\begin{aligned} 69 & \left\{\{1,0,0\},\left\{0,2\left(1+c_{1}\right), 0\right\}\right. \\
& \left.\left\{2\left(1+c_{1}\right)\left(3+d_{1}+d_{2}\right), 2\left(1+c_{1}\right)\left(5+2 d_{1}\right), 4\left(1+c_{1}\right)\left(3+d_{1}+d_{2}\right)\right\}\right\}\end{aligned}$ & 44 \\
\hline $15 \mathrm{Amm} 2$ & $3 m_{x}$ & $70\{\{1,0,0\},\{0,2,0\},\{4,6,8\}\}$ & \\
\hline $15 \mathrm{Amm} 2$ & $3 m_{x}$ & $71\left\{\{1,0,0\},\left\{0,2\left(1+c_{1}\right), 0\right\},\left\{2\left(1+c_{1}\right), 2\left(1+c_{1}\right), 4\left(1+c_{1}\right)\right\}\right\}$ & \\
\hline $15 A m m 2$ & $3 m_{x}$ & $72\left\{\{1,0,0\},\left\{0,2\left(1+c_{1}\right), 0\right\},\left\{6\left(1+c_{1}\right), 10\left(1+c_{1}\right), 12\left(1+c_{1}\right)\right\}\right\}$ & \\
\hline $15 A m m 2$ & $3 m_{x}$ & $73\left\{\{1,0,0\},\left\{0,2\left(2+c_{1}\right), 0\right\},\left\{4\left(2+c_{1}\right), 6\left(2+c_{1}\right), 8\left(2+c_{1}\right)\right\}\right\}$ & \\
\hline $15 \mathrm{Amm} 2$ & $3 m_{x}$ & $\begin{aligned} 74 & \left\{\{1,0,0\},\left\{0,2\left(1+c_{1}\right), 0\right\}\right. \\
& \left.\left\{2\left(1+c_{1}\right)\left(2+c_{2}\right), 2\left(1+c_{1}\right), 4\left(1+c_{1}\right)\left(2+c_{2}\right)\right\}\right\}\end{aligned}$ & \\
\hline 15 Amm 2 & $3 m_{x}$ & $\begin{aligned} 75\left\{\{1,0,0\},\left\{0,2\left(1+c_{1}\right), 0\right\}\right. \\
\left.\left\{2\left(1+c_{1}\right)\left(4+c_{2}\right), 2\left(1+c_{1}\right)\left(7+2 c_{2}\right), 4\left(1+c_{1}\right)\left(4+c_{2}\right)\right\}\right\}\end{aligned}$ & \\
\hline $15 \mathrm{Amm} 2$ & $3 m_{x}$ & $\begin{aligned} 76 & \left\{\{1,0,0\},\left\{0,2\left(1+c_{1}\right), 0\right\}\right. \\
& \left.\left\{2\left(1+c_{1}\right)\left(4+d_{1}+d_{2}\right), 2\left(1+c_{1}\right)\left(3+2 d_{1}\right), 4\left(1+c_{1}\right)\left(4+d_{1}+d_{2}\right)\right\}\right\}\end{aligned}$ & 46 \\
\hline
\end{tabular}


Table A.1 - continued from previous page

\begin{tabular}{|c|c|c|c|}
\hline $\mathrm{ACC}$ & $P_{N}$ & $B_{N}$ matrix row by row & $\mathrm{c} \#$ \\
\hline 15 Amm2 & $3 m_{x}$ & $\begin{aligned} 77\{ & \{1,0,0\},\left\{0,2\left(1+c_{1}\right), 0\right\} \\
& \left.\left\{2\left(1+c_{1}\right)\left(4+d_{1}+d_{2}\right), 2\left(1+c_{1}\right)\left(5+2 d_{1}\right), 4\left(1+c_{1}\right)\left(4+d_{1}+d_{2}\right)\right\}\right\}\end{aligned}$ & 48 \\
\hline $15 \mathrm{Amm} 2$ & $4 m_{y}$ & $1\left\{\left\{1+c_{1}, 0,0\right\},\{0,1,0\},\left\{0, c_{2}, 1+c_{2}\right\}\right\}$ & \\
\hline $15 \mathrm{Amm} 2$ & $4 m_{y}$ & $2\left\{\left\{1+c_{1}, 0,0\right\},\{0,1,0\},\left\{1+c_{2}, 1+2 c_{2}, 2\left(1+c_{2}\right)\right\}\right\}$ & \\
\hline 15 Amm 2 & $5 m m 2_{z}$ & $1\left\{\{1,0,0\},\{0,1,0\},\left\{1+c_{1}, 1+2 c_{1}, 2+2 c_{1}\right\}\right\}$ & \\
\hline 15 Amm 2 & $5 m m 2_{z}$ & $2\left\{\{1,0,0\},\{0,1,0\},\left\{0, c_{1}, 1+c_{1}\right\}\right\}$ & \\
\hline 15 Amm 2 & $5 m m 2_{z}$ & $3\left\{\{2,0,0\},\{0,1,0\},\left\{0, c_{1}, 1+c_{1}\right\}\right\}$ & \\
\hline 16 Fmm2 & $22 z$ & $1\left\{\{1,0,0\},\left\{c_{1}, 1+c_{1}, 0\right\},\{1,0,2\}\right\}$ & \\
\hline 16 F $m m 2$ & $22 z$ & $2\left\{\{1,0,0\},\left\{c_{1}, 1+c_{1}, 0\right\},\{0,1,2\}\right\}$ & \\
\hline $16 F m m 2$ & $22_{z}$ & $3\left\{\{1,0,0\},\left\{c_{1}, 1+c_{1}, 0\right\},\{0,0,1\}\right\}$ & \\
\hline 16 Fmm2 & $3 m_{x}$ & $1\left\{\{1,0,0\},\left\{c_{2}, 1+c_{2}, 0\right\},\left\{c_{1}, 0,1+c_{1}\right\}\right\}$ & \\
\hline 16 Fmm2 & $3 m_{x}$ & $2\left\{\{1,0,0\},\left\{c_{1}, 1+c_{1}, 0\right\},\left\{c_{2}, 1+c_{2}, 2\left(1+c_{2}\right)\right\}\right\}$ & \\
\hline 16 Fmm2 & $4 m_{y}$ & $1\left\{\{1,0,0\},\left\{\left(1+c_{1}\right) c_{2}, 1+c_{2}+c_{1} c_{2}, 0\right\},\left\{0, c_{1}, 1+c_{1}\right\}\right\}$ & \\
\hline 16 Fmm2 & $4 m_{y}$ & $\begin{aligned} 2 & \left\{\{1,0,0\},\left\{\left(1+c_{1}\right)\left(1+2 c_{2}\right), 2+c_{1}+2 c_{2}+2 c_{1} c_{2}, 0\right\}\right. \\
& \left.\left\{0,1+2 c_{1}, 2\left(1+c_{1}\right)\right\}\right\}\end{aligned}$ & \\
\hline 16 Fmm2 & $4 m_{y}$ & $3\left\{\{1,0,0\},\left\{c_{1}, 1+c_{1}, 0\right\},\left\{1,0,2\left(1+c_{1}\right)\right\}\right\}$ & \\
\hline 16 Fmm2 & $4 m_{y}$ & $4\left\{\{1,0,0\},\left\{c_{1}, 1+c_{1}, 0\right\},\left\{2\left(1+c_{2}\right), 1+2 c_{2}, 2\left(2+c_{1}+2 c_{2}\right)\right\}\right\}$ & \\
\hline 16 Fmm2 & $4 m_{y}$ & $5\left\{\{1,0,0\},\left\{c_{1}, 1+c_{1}, 0\right\},\left\{3+2 c_{2}, 2\left(1+c_{2}\right), 2\left(3+c_{1}+2 c_{2}\right)\right\}\right\}$ & \\
\hline 16 Fmm2 & $4 m_{y}$ & $\begin{aligned} 6 & \left\{\{1,0,0\},\left\{1+2 c_{3}+2 c_{1}\left(1+c_{2}+c_{3}\right), 2\left(1+c_{3}+c_{1}\left(1+c_{2}+c_{3}\right)\right), 0\right\}\right. \\
& \left.\left\{1+2 c_{2}, 2 c_{2}, 2\left(1+c_{2}+c_{3}\right)\right\}\right\}\end{aligned}$ & \\
\hline 16 Fmm2 & $4 m_{y}$ & $\begin{aligned} 7 & \left\{\{1,0,0\},\left\{2+c_{2}+3 c_{3}+2 c_{1}\left(1+c_{2}+c_{3}\right)\right.\right. \\
& \left.\left.3+c_{2}+3 c_{3}+2 c_{1}\left(1+c_{2}+c_{3}\right), 0\right\},\left\{1+2 c_{2}, 2 c_{2}, 2\left(1+c_{2}+c_{3}\right)\right\}\right\}\end{aligned}$ & \\
\hline 16 Fmm2 & $4 m_{y}$ & $\begin{aligned} 8 & \left\{\{1,0,0\},\left\{2\left(1+c_{3}+c_{1}\left(2+c_{2}+c_{3}\right)\right), 3+2 c_{3}+2 c_{1}\left(2+c_{2}+c_{3}\right), 0\right\}\right. \\
& \left.\left\{2\left(1+c_{2}\right), 1+2 c_{2}, 2\left(2+c_{2}+c_{3}\right)\right\}\right\}\end{aligned}$ & \\
\hline 16 Fmm2 & $4 m_{y}$ & $\begin{aligned} 9 & \left\{\{1,0,0\},\left\{4+c_{2}+3 c_{3}+2 c_{1}\left(2+c_{2}+c_{3}\right)\right.\right. \\
& \left.\left.5+c_{2}+3 c_{3}+2 c_{1}\left(2+c_{2}+c_{3}\right), 0\right\},\left\{2\left(1+c_{2}\right), 1+2 c_{2}, 2\left(2+c_{2}+c_{3}\right)\right\}\right\}\end{aligned}$ & \\
\hline 16 Fmm2 & $4 m_{y}$ & $\begin{aligned} 10 & \left\{\{1,0,0\},\left\{1+2 c_{3}+c_{1}\left(3+2 c_{2}+2 c_{3}\right), 2\left(1+c_{3}\right)+c_{1}\left(3+2 c_{2}+2 c_{3}\right), 0\right\}\right. \\
& \left.\left\{2\left(1+c_{2}\right), 1+2 c_{2}, 3+2 c_{2}+2 c_{3}\right\}\right\}\end{aligned}$ & \\
\hline
\end{tabular}


Table A.1 - continued from previous page

\begin{tabular}{|c|c|c|c|}
\hline ACC & $P_{N}$ & $B_{N}$ matrix row by row & $\mathrm{c \#}$ \\
\hline $16 \mathrm{Fmm} 2$ & $4 m_{y}$ & $\begin{aligned} 11 & \left\{\{1,0,0\},\left\{2\left(1+c_{3}\right)+c_{1}\left(3+2 c_{2}+2 c_{3}\right), 3+2 c_{3}+c_{1}\left(3+2 c_{2}+2 c_{3}\right), 0\right\}\right. \\
& \left.\left\{1+2 c_{2}, 2 c_{2}, 3+2 c_{2}+2 c_{3}\right\}\right\}\end{aligned}$ & \\
\hline $16 F m m 2$ & $5 m m 2_{z}$ & $1\left\{\{1,0,0\},\left\{c_{1}, 1+c_{1}, 0\right\},\{1,0,2\}\right\}$ & \\
\hline 16 Fmm2 & $5 m m 2_{z}$ & $2\left\{\{1,0,0\},\left\{c_{1}, 1+c_{1}, 0\right\},\{0,1,2\}\right\}$ & \\
\hline $16 \mathrm{Fmm} 2$ & $5 m m 2_{z}$ & $3\left\{\{1,0,0\},\left\{c_{1}, 1+c_{1}, 0\right\},\{0,0,1\}\right\}$ & \\
\hline $17 \operatorname{Imm} 2$ & $22_{z}$ & $1\{\{1,0,0\},\{1,2,0\},\{0,0,2\}\}$ & \\
\hline $17 \operatorname{Imm} 2$ & $22_{z}$ & $2\{\{1,0,0\},\{0,1,0\},\{0,0,1\}\}$ & \\
\hline $17 \operatorname{Imm} 2$ & $22_{z}$ & $3\left\{\{1,0,0\},\{1,2,0\},\left\{2,2,2\left(2+c_{1}\right)\right\}\right\}$ & \\
\hline $17 \operatorname{Imm} 2$ & $22_{z}$ & $4\left\{\{1,0,0\},\{0,1,0\},\left\{3+2 c_{1}, 3+2 c_{1}, 4\left(1+c_{1}\right)\right\}\right\}$ & \\
\hline $17 \operatorname{Imm} 2$ & $22_{z}$ & $5\left\{\{1,0,0\},\{0,1,0\},\left\{1,1,2+c_{1}\right\}\right\}$ & \\
\hline $17 \operatorname{Imm} 2$ & $3 m_{x}$ & $1\{\{1,0,0\},\{0,1,0\},\{0,0,1\}\}$ & \\
\hline $17 \operatorname{Imm} 2$ & $3 m_{x}$ & $2\left\{\left\{2+c_{1}, 0,0\right\},\{0,1,0\},\left\{0,1,2+c_{1}\right\}\right\}$ & \\
\hline $17 \operatorname{Imm} 2$ & $3 m_{x}$ & $3\left\{\left\{2+c_{1}, 0,0\right\},\{0,1,0\},\left\{\left(2+c_{1}\right)\left(1+c_{2}\right), 1,\left(2+c_{1}\right)\left(2+c_{2}\right)\right\}\right\}$ & \\
\hline $17 \operatorname{Imm} 2$ & $3 m_{x}$ & $4\left\{\left\{1+c_{1}, 0,0\right\},\{0,1,0\},\left\{\left(1+c_{1}\right)\left(1+d_{1}\right), 1,\left(1+c_{1}\right)\left(3+d_{1}+d_{2}\right)\right\}\right\}$ & 38 \\
\hline $17 \operatorname{Imm} 2$ & $3 m_{x}$ & $5\left\{\left\{1+c_{1}, 0,0\right\},\{0,1,0\},\left\{1+c_{1}, 1,2\left(1+c_{1}\right)\right\}\right\}$ & \\
\hline $17 \operatorname{Imm} 2$ & $3 m_{x}$ & $6\left\{\left\{1+c_{1}, 0,0\right\},\{0,1,0\},\left\{1+c_{1}, 1,\left(1+c_{1}\right)\left(3+c_{2}\right)\right\}\right\}$ & \\
\hline $17 \operatorname{Imm} 2$ & $3 m_{x}$ & $7\left\{\left\{1+c_{1}, 0,0\right\},\{0,1,0\},\left\{\left(1+c_{1}\right)\left(2+d_{1}\right), 1,\left(1+c_{1}\right)\left(3+d_{1}+d_{2}\right)\right\}\right\}$ & 42 \\
\hline $17 \operatorname{Imm} 2$ & $4 m_{y}$ & $1\{\{1,0,0\},\{0,1,0\},\{0,0,1\}\}$ & \\
\hline $17 \operatorname{Imm} 2$ & $4 m_{y}$ & $2\{\{1,0,0\},\{0,1,0\},\{1,1,3\}\}$ & \\
\hline $17 \operatorname{Imm} 2$ & $4 m_{y}$ & $3\{\{1,0,0\},\{0,2,0\},\{1,2,8\}\}$ & \\
\hline $17 \operatorname{Imm} 2$ & $4 m_{y}$ & $4\left\{\{1,0,0\},\{0,1,0\},\left\{1,1,4+c_{1}\right\}\right\}$ & \\
\hline $17 \operatorname{Imm} 2$ & $4 m_{y}$ & $5\left\{\{1,0,0\},\left\{0,2+c_{1}, 0\right\},\left\{1,0,2+c_{1}\right\}\right\}$ & \\
\hline $17 \operatorname{Imm} 2$ & $4 m_{y}$ & $6\left\{\{1,0,0\},\left\{0,2+c_{1}, 0\right\},\left\{1,2+c_{1}, 3\left(2+c_{1}\right)\right\}\right\}$ & \\
\hline $17 \operatorname{Imm} 2$ & $4 m_{y}$ & $7\left\{\{1,0,0\},\left\{0,3+c_{1}, 0\right\},\left\{1,3+c_{1}, 4\left(3+c_{1}\right)\right\}\right\}$ & \\
\hline $17 \operatorname{Imm} 2$ & $4 m_{y}$ & $8\left\{\{1,0,0\},\left\{0,2+c_{1}, 0\right\},\left\{1,\left(2+c_{1}\right)\left(1+c_{2}\right),\left(2+c_{1}\right)\left(2+c_{2}\right)\right\}\right\}$ & \\
\hline $17 \operatorname{Imm} 2$ & $4 m_{y}$ & $9\left\{\{1,0,0\},\{0,1,0\},\left\{1,2+d_{1}, 5+d_{1}+d_{2}\right\}\right\}$ & 17 \\
\hline $17 \operatorname{Imm} 2$ & $4 m_{y}$ & $10\left\{\{1,0,0\},\{0,2,0\},\left\{1,2\left(1+d_{1}\right), 2\left(5+d_{1}+d_{2}\right)\right\}\right\}$ & 16 \\
\hline $17 \operatorname{Imm} 2$ & $4 m_{y}$ & $11\left\{\{1,0,0\},\{0,2,0\},\left\{1,2\left(2+d_{1}\right), 2\left(5+d_{1}+d_{2}\right)\right\}\right\}$ & 17 \\
\hline
\end{tabular}


Table A.1 - continued from previous page

\begin{tabular}{|c|c|c|c|}
\hline ACC & $P_{N}$ & $B_{N}$ matrix row by row & $\mathrm{c} \#$ \\
\hline $17 \operatorname{Imm} 2$ & $4 m_{y}$ & $12\left\{\{1,0,0\},\left\{0,3+c_{1}, 0\right\},\left\{1,\left(3+c_{1}\right)\left(1+d_{1}\right),\left(3+c_{1}\right)\left(5+d_{1}+d_{2}\right)\right\}\right\}$ & 49 \\
\hline $17 \operatorname{Imm} 2$ & $4 m_{y}$ & $13\left\{\{1,0,0\},\left\{0,3+c_{1}, 0\right\},\left\{1,\left(3+c_{1}\right)\left(2+d_{1}\right),\left(3+c_{1}\right)\left(5+d_{1}+d_{2}\right)\right\}\right\}$ & 50 \\
\hline $17 \operatorname{Imm} 2$ & $4 m_{y}$ & $14\left\{\{1,0,0\},\{0,1,0\},\left\{1,2+c_{1}, 3+c_{1}\right\}\right\}$ & \\
\hline $17 \operatorname{Imm} 2$ & $4 m_{y}$ & $15\left\{\{1,0,0\},\left\{0,1+c_{1}, 0\right\},\left\{1,1+c_{1}, 2\left(1+c_{1}\right)\right\}\right\}$ & \\
\hline $17 \operatorname{Imm} 2$ & $4 m_{y}$ & $16\left\{\{1,0,0\},\left\{0,1+c_{1}, 0\right\},\left\{1,1+c_{1},\left(1+c_{1}\right)\left(3+c_{2}\right)\right\}\right\}$ & \\
\hline $17 \operatorname{Imm} 2$ & $4 m_{y}$ & $17\left\{\{1,0,0\},\left\{0,2+c_{1}, 0\right\},\left\{1,\left(2+c_{1}\right)\left(2+c_{2}\right),\left(2+c_{1}\right)\left(3+c_{2}\right)\right\}\right\}$ & \\
\hline $17 \operatorname{Imm} 2$ & $4 m_{y}$ & $18\left\{\{1,0,0\},\{0,1,0\},\left\{1,3+d_{1}, 5+d_{1}+d_{2}\right\}\right\}$ & 18 \\
\hline $17 \operatorname{Imm} 2$ & $4 m_{y}$ & $19\left\{\{1,0,0\},\left\{0,2+c_{1}, 0\right\},\left\{1,\left(2+c_{1}\right)\left(2+d_{1}\right),\left(2+c_{1}\right)\left(5+d_{1}+d_{2}\right)\right\}\right\}$ & 51 \\
\hline $17 \operatorname{Imm} 2$ & $4 m_{y}$ & $20\left\{\{1,0,0\},\left\{0,2+c_{1}, 0\right\},\left\{1,\left(2+c_{1}\right)\left(3+d_{1}\right),\left(2+c_{1}\right)\left(5+d_{1}+d_{2}\right)\right\}\right\}$ & 52 \\
\hline $17 \operatorname{Imm} 2$ & $5 m m 2_{z}$ & $1\{\{1,0,0\},\{0,1,0\},\{0,0,1\}\}$ & \\
\hline $17 \operatorname{Imm} 2$ & $5 m m 2_{z}$ & $2\left\{\{1,0,0\},\{0,1,0\},\left\{1,1,2+c_{1}\right\}\right\}$ & \\
\hline 18 Pmmm & $2 \overline{1}$ & $1\{\{1,0,0\},\{0,1,0\},\{0,0,1\}\}$ & \\
\hline 18 Pmmm & $2 \overline{1}$ & $2\{\{1,0,0\},\{0,1,0\},\{1,1,2\}\}$ & \\
\hline 18 Pmmm & $2 \overline{1}$ & $3\{\{1,0,0\},\{0,1,0\},\{1,0,2\}\}$ & \\
\hline 18 Pmmm & $2 \overline{1}$ & $4\{\{1,0,0\},\{0,1,0\},\{0,1,2\}\}$ & \\
\hline 18 Pmmm & $2 \overline{1}$ & $5\{\{1,0,0\},\{0,1,0\},\{0,0,2\}\}$ & \\
\hline 18 Pmmm & $2 \overline{1}$ & $6\{\{1,0,0\},\{1,2,0\},\{0,0,1\}\}$ & \\
\hline 18 Pmmm & $2 \overline{1}$ & $7\{\{1,0,0\},\{0,2,0\},\{0,0,1\}\}$ & \\
\hline 18 Pmmm & $2 \overline{1}$ & $8\{\{1,0,0\},\{1,2,0\},\{1,0,2\}\}$ & \\
\hline 18 Pmmm & $2 \overline{1}$ & $9\{\{1,0,0\},\{1,2,0\},\{0,0,2\}\}$ & \\
\hline 18 Pmmm & $2 \overline{1}$ & $10\{\{1,0,0\},\{0,2,0\},\{1,0,2\}\}$ & \\
\hline 18 Pmmm & $2 \overline{1}$ & $11\{\{1,0,0\},\{0,2,0\},\{0,0,2\}\}$ & \\
\hline 18 Pmmm & $2 \overline{1}$ & $12\{\{2,0,0\},\{0,1,0\},\{0,0,1\}\}$ & \\
\hline 18 Pmmm & $2 \overline{1}$ & $13\{\{2,0,0\},\{0,1,0\},\{0,1,2\}\}$ & \\
\hline 18 Pmmm & $2 \overline{1}$ & $14\{\{2,0,0\},\{0,1,0\},\{0,0,2\}\}$ & \\
\hline 18 Pmmm & $2 \overline{1}$ & $15\{\{2,0,0\},\{0,2,0\},\{0,0,1\}\}$ & \\
\hline 18 Pmmm & $2 \overline{1}$ & $16\{\{2,0,0\},\{0,2,0\},\{0,0,2\}\}$ & \\
\hline 18 Pmmm & $32 z$ & $1\left\{\{1,0,0\},\{0,1,0\},\left\{1+c_{1}, 1+c_{1}, 2+2 c_{1}\right\}\right\}$ & \\
\hline
\end{tabular}


Table A.1 - continued from previous page

\begin{tabular}{|c|c|c|c|}
\hline ACC & $P_{N}$ & $B_{N}$ matrix row by row & $\mathrm{c \#}$ \\
\hline 18 Pmmm & $32 z$ & $2\left\{\{1,0,0\},\{0,1,0\},\left\{1+c_{1}, 0,2+2 c_{1}\right\}\right\}$ & \\
\hline 18 Pmmm & $32_{z}$ & $3\left\{\{1,0,0\},\{0,1,0\},\left\{0,1+c_{1}, 2+2 c_{1}\right\}\right\}$ & \\
\hline 18 Pmmm & $32_{z}$ & $4\left\{\{1,0,0\},\{0,1,0\},\left\{0,0,1+c_{1}\right\}\right\}$ & \\
\hline 18 Pmmm & $32_{z}$ & $5\left\{\{1,0,0\},\{1,2,0\},\left\{1+c_{1}, 0,2+2 c_{1}\right\}\right\}$ & \\
\hline 18 Pmmm & $32_{z}$ & $6\left\{\{1,0,0\},\{1,2,0\},\left\{0,0,1+c_{1}\right\}\right\}$ & \\
\hline 18 Pmmm & $32 z$ & $7\left\{\{1,0,0\},\{0,2,0\},\left\{1+c_{1}, 0,2+2 c_{1}\right\}\right\}$ & \\
\hline $18 \mathrm{Pmmm}$ & $32 z$ & $8\left\{\{1,0,0\},\{0,2,0\},\left\{0,0,1+c_{1}\right\}\right\}$ & \\
\hline 18 Pmmm & $32 z$ & $9\left\{\{2,0,0\},\{0,1,0\},\left\{0,1+c_{1}, 2+2 c_{1}\right\}\right\}$ & \\
\hline 18 Pmmm & $32 z$ & $10\left\{\{2,0,0\},\{0,1,0\},\left\{0,0,1+c_{1}\right\}\right\}$ & \\
\hline 18 Pmmm & $32 z$ & $11\left\{\{2,0,0\},\{0,2,0\},\left\{0,0,1+c_{1}\right\}\right\}$ & \\
\hline 18 Pmmm & $42 y$ & $1\left\{\{1,0,0\},\left\{1+c_{1}, 2+2 c_{1}, 0\right\},\{0,0,1\}\right\}$ & \\
\hline 18 Pmmm & $42 y$ & $2\left\{\{1,0,0\},\left\{0,1+c_{1}, 0\right\},\{0,0,1\}\right\}$ & \\
\hline 18 Pmmm & $42 y$ & $3\left\{\{1,0,0\},\left\{1+c_{1}, 2+2 c_{1}, 0\right\},\{1,0,2\}\right\}$ & \\
\hline 18 Pmmm & $42 y$ & $4\left\{\{1,0,0\},\left\{1+c_{1}, 2+2 c_{1}, 0\right\},\{0,0,2\}\right\}$ & \\
\hline 18 Pmmm & $42 y$ & $5\left\{\{1,0,0\},\left\{0,1+c_{1}, 0\right\},\{1,0,2\}\right\}$ & \\
\hline 18 Pmmm & $42 y$ & $6\left\{\{1,0,0\},\left\{0,1+c_{1}, 0\right\},\{1,1,2\}\right\}$ & \\
\hline $18 \mathrm{Pmmm}$ & $42 y$ & $7\left\{\{1,0,0\},\left\{0,1+c_{1}, 0\right\},\{0,0,2\}\right\}$ & \\
\hline 18 Pmmm & $42 y$ & $8\left\{\{1,0,0\},\left\{0,1+c_{1}, 0\right\},\{0,1,2\}\right\}$ & \\
\hline 18 Pmmm & $42 y$ & $9\left\{\{2,0,0\},\left\{0,1+c_{1}, 0\right\},\{0,0,1\}\right\}$ & \\
\hline 18 Pmmm & $42 y$ & $10\left\{\{2,0,0\},\left\{0,1+c_{1}, 0\right\},\{0,0,2\}\right\}$ & \\
\hline $18 \mathrm{Pmmm}$ & $42 y$ & $11\left\{\{2,0,0\},\left\{0,1+c_{1}, 0\right\},\{0,1,2\}\right\}$ & \\
\hline 18 Pmmm & $5 m_{x}$ & $1\left\{\{1,0,0\},\left\{1+c_{1}, 2\left(1+c_{1}\right), 0\right\},\left\{1+c_{2}, 0,2\left(1+c_{2}\right)\right\}\right\}$ & \\
\hline 18 Pmmm & $5 m_{x}$ & $2\left\{\{1,0,0\},\left\{1+c_{1}, 2\left(1+c_{1}\right), 0\right\},\left\{0,0,1+c_{2}\right\}\right\}$ & \\
\hline 18 Pmmm & $5 m_{x}$ & $3\left\{\{1,0,0\},\left\{0,1+c_{1}, 0\right\},\left\{0,0,1+c_{2}\right\}\right\}$ & \\
\hline 18 Pmmm & $5 m_{x}$ & $4\left\{\{1,0,0\},\left\{0,1+c_{1}, 0\right\},\left\{0,1+c_{2}, 2\left(1+c_{2}\right)\right\}\right\}$ & \\
\hline 18 Pmmm & $5 m_{x}$ & $5\left\{\{2,0,0\},\left\{0,1+c_{1}, 0\right\},\left\{0,0,1+c_{2}\right\}\right\}$ & \\
\hline $18 \mathrm{Pmmm}$ & $5 m_{x}$ & $6\left\{\{2,0,0\},\left\{0,1+c_{1}, 0\right\},\left\{0,1+c_{2}, 2\left(1+c_{2}\right)\right\}\right\}$ & \\
\hline 18 Pmmm & $5 m_{x}$ & $7\left\{\{1,0,0\},\left\{0,1+c_{1}, 0\right\},\left\{1+c_{2}, 0,2\left(1+c_{2}\right)\right\}\right\}$ & \\
\hline
\end{tabular}


Table A.1 - continued from previous page

\begin{tabular}{|c|c|c|c|}
\hline ACC & $P_{N}$ & $B_{N}$ matrix row by row & $\mathrm{c \#}$ \\
\hline 18 Pmmm & $5 m_{x}$ & $8\left\{\{1,0,0\},\left\{0,1+c_{1}, 0\right\},\left\{2\left(1+c_{2}\right), 2\left(1+c_{2}\right), 4\left(1+c_{2}\right)\right\}\right\}$ & \\
\hline 18 Pmmm & $5 m_{x}$ & $9\left\{\{1,0,0\},\left\{0,1+c_{1}, 0\right\},\left\{3+2 c_{2}, 3+2 c_{2}, 6+4 c_{2}\right\}\right\}$ & \\
\hline 18 Pmmm & $5 m_{x}$ & $10\left\{\{1,0,0\},\left\{0,1+c_{1}, 0\right\},\left\{1+2 c_{2}, 1+2 c_{2}, 2+4 c_{2}\right\}\right\}$ & \\
\hline 18 Pmmm & $5 m_{x}$ & $11\left\{\{1,0,0\},\left\{0,1+c_{1}, 0\right\},\left\{2\left(1+c_{2}\right), 2\left(1+c_{2}\right), 4\left(1+c_{2}\right)\right\}\right\}$ & \\
\hline 18 Pmmm & $62_{x}$ & $1\left\{\left\{1+c_{1}, 0,0\right\},\{0,1,0\},\{0,0,1\}\right\}$ & \\
\hline 18 Pmmm & $62_{x}$ & $2\left\{\left\{1+c_{1}, 0,0\right\},\{0,1,0\},\{0,1,2\}\right\}$ & \\
\hline $18 \mathrm{Pmmm}$ & $62_{x}$ & $3\left\{\left\{1+c_{1}, 0,0\right\},\{0,1,0\},\{1,1,2\}\right\}$ & \\
\hline 18 Pmmm & $62_{x}$ & $4\left\{\left\{1+c_{1}, 0,0\right\},\{0,1,0\},\{0,0,2\}\right\}$ & \\
\hline 18 Pmmm & $62_{x}$ & $5\left\{\left\{1+c_{1}, 0,0\right\},\{0,1,0\},\{1,0,2\}\right\}$ & \\
\hline 18 Pmmm & $62_{x}$ & $6\left\{\left\{1+c_{1}, 0,0\right\},\{0,2,0\},\{0,0,1\}\right\}$ & \\
\hline 18 Pmmm & $62_{x}$ & $7\left\{\left\{1+c_{1}, 0,0\right\},\{1,2,0\},\{0,0,1\}\right\}$ & \\
\hline 18 Pmmm & $62_{x}$ & $8\left\{\left\{1+c_{1}, 0,0\right\},\{0,2,0\},\{0,0,2\}\right\}$ & \\
\hline 18 Pmmm & $62_{x}$ & $9\left\{\left\{1+c_{1}, 0,0\right\},\{0,2,0\},\{1,0,2\}\right\}$ & \\
\hline 18 Pmmm & $62_{x}$ & $10\left\{\left\{1+c_{1}, 0,0\right\},\{1,2,0\},\{0,0,2\}\right\}$ & \\
\hline 18 Pmmm & $62_{x}$ & $11\left\{\left\{1+c_{1}, 0,0\right\},\{1,2,0\},\{1,0,2\}\right\}$ & \\
\hline 18 Pmmm & $7 m_{y}$ & $1\left\{\left\{1+c_{1}, 0,0\right\},\{0,1,0\},\left\{0,1+c_{2}, 2\left(1+c_{2}\right)\right\}\right\}$ & \\
\hline $18 \mathrm{Pmmm}$ & $7 m_{y}$ & $2\left\{\left\{1+c_{1}, 0,0\right\},\{0,1,0\},\left\{2\left(1+c_{2}\right), 2\left(1+c_{2}\right), 4\left(1+c_{2}\right)\right\}\right\}$ & \\
\hline 18 Pmmm & $7 m_{y}$ & $3\left\{\left\{1+c_{1}, 0,0\right\},\{0,1,0\},\left\{1+2 c_{2}, 1+2 c_{2}, 2+4 c_{2}\right\}\right\}$ & \\
\hline 18 Pmmm & $7 m_{y}$ & $4\left\{\left\{1+c_{1}, 0,0\right\},\{0,1,0\},\left\{0,0,2\left(1+c_{2}\right)\right\}\right\}$ & \\
\hline 18 Pmmm & $7 m_{y}$ & $5\left\{\left\{1+c_{1}, 0,0\right\},\{0,1,0\},\left\{2\left(1+c_{2}\right), 0,4\left(1+c_{2}\right)\right\}\right\}$ & \\
\hline 18 Pmmm & $7 m_{y}$ & $6\left\{\left\{1+c_{1}, 0,0\right\},\{0,1,0\},\left\{0,0,1+2 c_{2}\right\}\right\}$ & \\
\hline 18 Pmmm & $7 m_{y}$ & $7\left\{\left\{1+c_{1}, 0,0\right\},\{0,1,0\},\left\{1+2 c_{2}, 0,2+4 c_{2}\right\}\right\}$ & \\
\hline 18 Pmmm & $7 m_{y}$ & $8\left\{\left\{1+c_{1}, 0,0\right\},\{0,2,0\},\left\{0,0,1+c_{2}\right\}\right\}$ & \\
\hline 18 Pmmm & $7 m_{y}$ & $9\left\{\left\{1+c_{1}, 0,0\right\},\{0,2,0\},\left\{1+c_{2}, 0,2\left(1+c_{2}\right)\right\}\right\}$ & \\
\hline $18 \mathrm{Pmmm}$ & $7 m_{y}$ & $10\left\{\left\{1+c_{1}, 0,0\right\},\{1,2,0\},\left\{0,0,1+c_{2}\right\}\right\}$ & \\
\hline 18 Pmmm & $7 m_{y}$ & $11\left\{\left\{1+c_{1}, 0,0\right\},\{1,2,0\},\left\{1+c_{2}, 0,2\left(1+c_{2}\right)\right\}\right\}$ & \\
\hline 18 Pmmm & $8 m_{z}$ & $1\left\{\left\{1+c_{1}, 0,0\right\},\left\{0,1+c_{2}, 0\right\},\{0,0,1\}\right\}$ & \\
\hline 18 Pmmm & $8 m_{z}$ & $2\left\{\left\{1+c_{1}, 0,0\right\},\left\{1+c_{2}, 2\left(1+c_{2}\right), 0\right\},\{0,0,1\}\right\}$ & \\
\hline
\end{tabular}


Table A.1 - continued from previous page

\begin{tabular}{|c|c|c|c|}
\hline ACC & $P_{N}$ & $B_{N}$ matrix row by row & $\mathrm{c \#}$ \\
\hline 18 Pmmm & $8 m_{z}$ & $3\left\{\left\{1+c_{1}, 0,0\right\},\left\{0,1+c_{2}, 0\right\},\{0,0,2\}\right\}$ & \\
\hline 18 Pmmm & $8 m_{z}$ & $4\left\{\left\{1+c_{1}, 0,0\right\},\left\{1+c_{2}, 2\left(1+c_{2}\right), 0\right\},\{0,0,2\}\right\}$ & \\
\hline 18 Pmmm & $8 m_{z}$ & $5\left\{\left\{1+c_{1}, 0,0\right\},\left\{0,1+c_{2}, 0\right\},\{0,1,2\}\right\}$ & \\
\hline 18 Pmmm & $8 m_{z}$ & $6\left\{\left\{1+c_{1}, 0,0\right\},\left\{0,1+c_{2}, 0\right\},\{1,0,2\}\right\}$ & \\
\hline 18 Pmmm & $8 m_{z}$ & $7\left\{\left\{1+c_{1}, 0,0\right\},\left\{1+c_{2}, 2\left(1+c_{2}\right), 0\right\},\{1,0,2\}\right\}$ & \\
\hline 18 Pmmm & $8 m_{z}$ & $8\left\{\left\{1+c_{1}, 0,0\right\},\left\{0,1+c_{2}, 0\right\},\{1,1,2\}\right\}$ & \\
\hline $18 \mathrm{Pmmm}$ & $92 / m_{z}$ & $1\{\{1,0,0\},\{0,1,0\},\{0,0,1\}\}$ & \\
\hline 18 Pmmm & $92 / m_{z}$ & $2\{\{1,0,0\},\{0,1,0\},\{1,1,2\}\}$ & \\
\hline 18 Pmmm & $92 / m_{z}$ & $3\{\{1,0,0\},\{0,1,0\},\{1,0,2\}\}$ & \\
\hline 18 Pmmm & $92 / m_{z}$ & $4\{\{1,0,0\},\{0,1,0\},\{0,1,2\}\}$ & \\
\hline 18 Pmmm & $92 / m_{z}$ & $5\{\{1,0,0\},\{0,1,0\},\{0,0,2\}\}$ & \\
\hline 18 Pmmm & $92 / m_{z}$ & $6\{\{1,0,0\},\{1,2,0\},\{0,0,1\}\}$ & \\
\hline 18 Pmmm & $92 / m_{z}$ & $7\{\{1,0,0\},\{0,2,0\},\{0,0,1\}\}$ & \\
\hline 18 Pmmm & $92 / m_{z}$ & $8\{\{1,0,0\},\{1,2,0\},\{1,0,2\}\}$ & \\
\hline 18 Pmmm & $92 / m_{z}$ & $9\{\{1,0,0\},\{1,2,0\},\{0,0,2\}\}$ & \\
\hline 18 Pmmm & $92 / m_{z}$ & $10\{\{1,0,0\},\{0,2,0\},\{1,0,2\}\}$ & \\
\hline 18 Pmmm & $92 / m_{z}$ & $11\{\{1,0,0\},\{0,2,0\},\{0,0,2\}\}$ & \\
\hline 18 Pmmm & $92 / m_{z}$ & $12\{\{2,0,0\},\{0,1,0\},\{0,0,1\}\}$ & \\
\hline 18 Pmmm & $92 / m_{z}$ & $13\{\{2,0,0\},\{0,1,0\},\{0,1,2\}\}$ & \\
\hline 18 Pmmm & $92 / m_{z}$ & $14\{\{2,0,0\},\{0,1,0\},\{0,0,2\}\}$ & \\
\hline 18 Pmmm & $92 / m_{z}$ & $15\{\{2,0,0\},\{0,2,0\},\{0,0,1\}\}$ & \\
\hline 18 Pmmm & $92 / m_{z}$ & $16\{\{2,0,0\},\{0,2,0\},\{0,0,2\}\}$ & \\
\hline 18 Pmmm & $102 / m_{y}$ & $1\{\{1,0,0\},\{0,1,0\},\{0,0,1\}\}$ & \\
\hline 18 Pmmm & $102 / m_{y}$ & $2\{\{1,0,0\},\{0,1,0\},\{1,1,2\}\}$ & \\
\hline 18 Pmmm & $102 / m_{y}$ & $3\{\{1,0,0\},\{0,1,0\},\{1,0,2\}\}$ & \\
\hline 18 Pmmm & $102 / m_{y}$ & $4\{\{1,0,0\},\{0,1,0\},\{0,1,2\}\}$ & \\
\hline $18 \mathrm{Pmmm}$ & $102 / m_{y}$ & $5\{\{1,0,0\},\{0,1,0\},\{0,0,2\}\}$ & \\
\hline 18 Pmmm & $102 / m_{y}$ & $6\{\{1,0,0\},\{1,2,0\},\{0,0,1\}\}$ & \\
\hline
\end{tabular}


Table A.1 - continued from previous page

\begin{tabular}{|c|c|c|c|}
\hline ACC & $P_{N}$ & $B_{N}$ matrix row by row & $\mathrm{c \#}$ \\
\hline 18 Pmmm & $102 / m_{y}$ & $7\{\{1,0,0\},\{0,2,0\},\{0,0,1\}\}$ & \\
\hline 18 Pmmm & $102 / m_{y}$ & $8\{\{1,0,0\},\{1,2,0\},\{1,0,2\}\}$ & \\
\hline 18 Pmmm & $102 / m_{y}$ & $9\{\{1,0,0\},\{1,2,0\},\{0,0,2\}\}$ & \\
\hline 18 Pmmm & $102 / m_{y}$ & $10\{\{1,0,0\},\{0,2,0\},\{1,0,2\}\}$ & \\
\hline 18 Pmmm & $102 / m_{y}$ & $11\{\{1,0,0\},\{0,2,0\},\{0,0,2\}\}$ & \\
\hline 18 Pmmm & $102 / m_{y}$ & $12\{\{2,0,0\},\{0,1,0\},\{0,0,1\}\}$ & \\
\hline 18 Pmmm & $102 / m_{y}$ & $13\{\{2,0,0\},\{0,1,0\},\{0,1,2\}\}$ & \\
\hline 18 Pmmm & $102 / m_{y}$ & $14\{\{2,0,0\},\{0,1,0\},\{0,0,2\}\}$ & \\
\hline 18 Pmmm & $102 / m_{y}$ & $15\{\{2,0,0\},\{0,2,0\},\{0,0,1\}\}$ & \\
\hline 18 Pmmm & $102 / m_{y}$ & $16\{\{2,0,0\},\{0,2,0\},\{0,0,2\}\}$ & \\
\hline 18 Pmmm & $112 / m_{x}$ & $1\{\{1,0,0\},\{0,1,0\},\{0,0,1\}\}$ & \\
\hline 18 Pmmm & $112 / m_{x}$ & $2\{\{1,0,0\},\{0,1,0\},\{1,1,2\}\}$ & \\
\hline 18 Pmmm & $112 / m_{x}$ & $3\{\{1,0,0\},\{0,1,0\},\{1,0,2\}\}$ & \\
\hline 18 Pmmm & $112 / m_{x}$ & $4\{\{1,0,0\},\{0,1,0\},\{0,1,2\}\}$ & \\
\hline 18 Pmmm & $112 / m_{x}$ & $5\{\{1,0,0\},\{0,1,0\},\{0,0,2\}\}$ & \\
\hline 18 Pmmm & $112 / m_{x}$ & $6\{\{1,0,0\},\{1,2,0\},\{0,0,1\}\}$ & \\
\hline 18 Pmmm & $112 / m_{x}$ & $7\{\{1,0,0\},\{0,2,0\},\{0,0,1\}\}$ & \\
\hline 18 Pmmm & $112 / m_{x}$ & $8\{\{1,0,0\},\{1,2,0\},\{1,0,2\}\}$ & \\
\hline 18 Pmmm & $112 / m_{x}$ & $9\{\{1,0,0\},\{1,2,0\},\{0,0,2\}\}$ & \\
\hline 18 Pmmm & $112 / m_{x}$ & $10\{\{1,0,0\},\{0,2,0\},\{1,0,2\}\}$ & \\
\hline 18 Pmmm & $112 / m_{x}$ & $11\{\{1,0,0\},\{0,2,0\},\{0,0,2\}\}$ & \\
\hline 18 Pmmm & $112 / m_{x}$ & $12\{\{2,0,0\},\{0,1,0\},\{0,0,1\}\}$ & \\
\hline 18 Pmmm & $112 / m_{x}$ & $13\{\{2,0,0\},\{0,1,0\},\{0,1,2\}\}$ & \\
\hline 18 Pmmm & $112 / m_{x}$ & $14\{\{2,0,0\},\{0,1,0\},\{0,0,2\}\}$ & \\
\hline 18 Pmmm & $112 / m_{x}$ & $15\{\{2,0,0\},\{0,2,0\},\{0,0,1\}\}$ & \\
\hline 18 Pmmm & $112 / m_{x}$ & $16\{\{2,0,0\},\{0,2,0\},\{0,0,2\}\}$ & \\
\hline $18 \mathrm{Pmmm}$ & 12222 & $1\{\{1,0,0\},\{0,1,0\},\{0,0,1\}\}$ & \\
\hline 18 Pmmm & 12222 & $2\{\{1,0,0\},\{0,1,0\},\{1,1,2\}\}$ & \\
\hline
\end{tabular}


Table A.1 - continued from previous page

\begin{tabular}{|c|c|c|c|}
\hline ACC & $P_{N}$ & $B_{N}$ matrix row by row & $\mathrm{c \#}$ \\
\hline $18 \mathrm{Pmmm}$ & 12222 & $3\{\{1,0,0\},\{0,1,0\},\{1,0,2\}\}$ & \\
\hline 18 Pmmm & 12222 & $4\{\{1,0,0\},\{0,1,0\},\{0,1,2\}\}$ & \\
\hline 18 Pmmm & 12222 & $5\{\{1,0,0\},\{0,1,0\},\{0,0,2\}\}$ & \\
\hline 18 Pmmm & 12222 & $6\{\{1,0,0\},\{1,2,0\},\{0,0,1\}\}$ & \\
\hline 18 Pmmm & 12222 & $7\{\{1,0,0\},\{0,2,0\},\{0,0,1\}\}$ & \\
\hline 18 Pmmm & 12222 & $8\{\{1,0,0\},\{1,2,0\},\{1,0,2\}\}$ & \\
\hline 18 Pmmm & 12222 & $9\{\{1,0,0\},\{1,2,0\},\{0,0,2\}\}$ & \\
\hline 18 Pmmm & 12222 & $10\{\{1,0,0\},\{0,2,0\},\{1,0,2\}\}$ & \\
\hline 18 Pmmm & 12222 & $11\{\{1,0,0\},\{0,2,0\},\{0,0,2\}\}$ & \\
\hline 18 Pmmm & 12222 & $12\{\{2,0,0\},\{0,1,0\},\{0,0,1\}\}$ & \\
\hline 18 Pmmm & 12222 & $13\{\{2,0,0\},\{0,1,0\},\{0,1,2\}\}$ & \\
\hline $18 \mathrm{Pmmm}$ & 12222 & $14\{\{2,0,0\},\{0,1,0\},\{0,0,2\}\}$ & \\
\hline 18 Pmmm & 12222 & $15\{\{2,0,0\},\{0,2,0\},\{0,0,1\}\}$ & \\
\hline 18 Pmmm & 12222 & $16\{\{2,0,0\},\{0,2,0\},\{0,0,2\}\}$ & \\
\hline 18 Pmmm & $13 m m 2_{z}$ & $1\left\{\{1,0,0\},\{0,1,0\},\left\{1+c_{1}, 1+c_{1}, 2+2 c_{1}\right\}\right\}$ & \\
\hline 18 Pmmm & $13 m m 2_{z}$ & $2\left\{\{1,0,0\},\{0,1,0\},\left\{1+c_{1}, 0,2+2 c_{1}\right\}\right\}$ & \\
\hline $18 \mathrm{Pmmm}$ & $13 m m 2_{z}$ & $3\left\{\{1,0,0\},\{0,1,0\},\left\{0,1+c_{1}, 2+2 c_{1}\right\}\right\}$ & \\
\hline 18 Pmmm & $13 m m 2_{z}$ & $4\left\{\{1,0,0\},\{0,1,0\},\left\{0,0,1+c_{1}\right\}\right\}$ & \\
\hline 18 Pmmm & $13 m m 2_{z}$ & $5\left\{\{1,0,0\},\{1,2,0\},\left\{1+c_{1}, 0,2+2 c_{1}\right\}\right\}$ & \\
\hline 18 Pmmm & $13 m m 2_{z}$ & $6\left\{\{1,0,0\},\{1,2,0\},\left\{0,0,1+c_{1}\right\}\right\}$ & \\
\hline 18 Pmmm & $13 m m 2_{z}$ & $7\left\{\{1,0,0\},\{0,2,0\},\left\{1+c_{1}, 0,2+2 c_{1}\right\}\right\}$ & \\
\hline 18 Pmmm & $13 m m 2_{z}$ & $8\left\{\{1,0,0\},\{0,2,0\},\left\{0,0,1+c_{1}\right\}\right\}$ & \\
\hline 18 Pmmm & $13 m m 2_{z}$ & $9\left\{\{2,0,0\},\{0,1,0\},\left\{0,1+c_{1}, 2+2 c_{1}\right\}\right\}$ & \\
\hline 18 Pmmm & $13 m m 2_{z}$ & $10\left\{\{2,0,0\},\{0,1,0\},\left\{0,0,1+c_{1}\right\}\right\}$ & \\
\hline 18 Pmmm & $13 m m 2_{z}$ & $11\left\{\{2,0,0\},\{0,2,0\},\left\{0,0,1+c_{1}\right\}\right\}$ & \\
\hline 18 Pmmm & $14 m m 2_{y}$ & $1\left\{\{1,0,0\},\left\{1+c_{1}, 2+2 c_{1}, 0\right\},\{0,0,1\}\right\}$ & \\
\hline $18 \mathrm{Pmmm}$ & $14 m m 2_{y}$ & $2\left\{\{1,0,0\},\left\{0,1+c_{1}, 0\right\},\{0,0,1\}\right\}$ & \\
\hline 18 Pmmm & $14 m m 2_{y}$ & $3\left\{\{1,0,0\},\left\{1+c_{1}, 2+2 c_{1}, 0\right\},\{1,0,2\}\right\}$ & \\
\hline
\end{tabular}


Table A.1 - continued from previous page

\begin{tabular}{|c|c|c|c|}
\hline ACC & $P_{N}$ & $B_{N}$ matrix row by row & $\mathrm{c \#}$ \\
\hline 18 Pmmm & $14 m m 2_{y}$ & $4\left\{\{1,0,0\},\left\{1+c_{1}, 2+2 c_{1}, 0\right\},\{0,0,2\}\right\}$ & \\
\hline 18 Pmmm & $14 m m 2_{y}$ & $5\left\{\{1,0,0\},\left\{0,1+c_{1}, 0\right\},\{1,0,2\}\right\}$ & \\
\hline 18 Pmmm & $14 m m 2_{y}$ & $6\left\{\{1,0,0\},\left\{0,1+c_{1}, 0\right\},\{1,1,2\}\right\}$ & \\
\hline 18 Pmmm & $14 m m 2_{y}$ & $7\left\{\{1,0,0\},\left\{0,1+c_{1}, 0\right\},\{0,0,2\}\right\}$ & \\
\hline 18 Pmmm & $14 m m 2_{y}$ & $8\left\{\{1,0,0\},\left\{0,1+c_{1}, 0\right\},\{0,1,2\}\right\}$ & \\
\hline 18 Pmmm & $14 m m 2_{y}$ & $9\left\{\{2,0,0\},\left\{0,1+c_{1}, 0\right\},\{0,0,1\}\right\}$ & \\
\hline 18 Pmmm & $14 m m 2_{y}$ & $10\left\{\{2,0,0\},\left\{0,1+c_{1}, 0\right\},\{0,0,2\}\right\}$ & \\
\hline 18 Pmmm & $14 m m 2_{y}$ & $11\left\{\{2,0,0\},\left\{0,1+c_{1}, 0\right\},\{0,1,2\}\right\}$ & \\
\hline 18 Pmmm & $15 m m 2_{x}$ & $1\left\{\left\{1+c_{1}, 0,0\right\},\{0,1,0\},\{0,0,1\}\right\}$ & \\
\hline 18 Pmmm & $15 m m 2_{x}$ & $2\left\{\left\{1+c_{1}, 0,0\right\},\{0,1,0\},\{0,1,2\}\right\}$ & \\
\hline 18 Pmmm & $15 m m 2_{x}$ & $3\left\{\left\{1+c_{1}, 0,0\right\},\{0,1,0\},\{1,1,2\}\right\}$ & \\
\hline $18 \mathrm{Pmmm}$ & $15 m m 2_{x}$ & $4\left\{\left\{1+c_{1}, 0,0\right\},\{0,1,0\},\{0,0,2\}\right\}$ & \\
\hline 18 Pmmm & $15 m m 2_{x}$ & $5\left\{\left\{1+c_{1}, 0,0\right\},\{0,1,0\},\{1,0,2\}\right\}$ & \\
\hline 18 Pmmm & $15 m m 2_{x}$ & $6\left\{\left\{1+c_{1}, 0,0\right\},\{0,2,0\},\{0,0,1\}\right\}$ & \\
\hline 18 Pmmm & $15 m m 2_{x}$ & $7\left\{\left\{1+c_{1}, 0,0\right\},\{1,2,0\},\{0,0,1\}\right\}$ & \\
\hline 18 Pmmm & $15 m m 2_{x}$ & $8\left\{\left\{1+c_{1}, 0,0\right\},\{0,2,0\},\{0,0,2\}\right\}$ & \\
\hline 18 Pmmm & $15 m m 2_{x}$ & $9\left\{\left\{1+c_{1}, 0,0\right\},\{0,2,0\},\{1,0,2\}\right\}$ & \\
\hline 18 Pmmm & $15 m m 2_{x}$ & $10\left\{\left\{1+c_{1}, 0,0\right\},\{1,2,0\},\{0,0,2\}\right\}$ & \\
\hline 18 Pmmm & $15 m m 2_{x}$ & $11\left\{\left\{1+c_{1}, 0,0\right\},\{1,2,0\},\{1,0,2\}\right\}$ & \\
\hline 18 Pmmm & $16 \mathrm{mmm}$ & $1\{\{1,0,0\},\{0,1,0\},\{0,0,1\}\}$ & \\
\hline 18 Pmmm & $16 \mathrm{mmm}$ & $2\{\{1,0,0\},\{0,1,0\},\{1,1,2\}\}$ & \\
\hline 18 Pmmm & $16 \mathrm{mmm}$ & $3\{\{1,0,0\},\{0,1,0\},\{1,0,2\}\}$ & \\
\hline 18 Pmmm & $16 \mathrm{mmm}$ & $4\{\{1,0,0\},\{0,1,0\},\{0,1,2\}\}$ & \\
\hline 18 Pmmm & $16 \mathrm{mmm}$ & $5\{\{1,0,0\},\{0,1,0\},\{0,0,2\}\}$ & \\
\hline 18 Pmmm & $16 \mathrm{mmm}$ & $6\{\{1,0,0\},\{1,2,0\},\{0,0,1\}\}$ & \\
\hline 18 Pmmm & $16 \mathrm{mmm}$ & $7\{\{1,0,0\},\{0,2,0\},\{0,0,1\}\}$ & \\
\hline 18 Pmmm & $16 \mathrm{mmm}$ & $8\{\{1,0,0\},\{1,2,0\},\{1,0,2\}\}$ & \\
\hline 18 Pmmm & $16 \mathrm{mmm}$ & $9\{\{1,0,0\},\{1,2,0\},\{0,0,2\}\}$ & \\
\hline
\end{tabular}


Table A.1 - continued from previous page

\begin{tabular}{|c|c|c|c|}
\hline ACC & $P_{N}$ & $B_{N}$ matrix row by row & $\mathrm{c \#}$ \\
\hline $18 \mathrm{Pmmm}$ & $16 \mathrm{mmm}$ & $10\{\{1,0,0\},\{0,2,0\},\{1,0,2\}\}$ & \\
\hline 18 Pmmm & $16 \mathrm{mmm}$ & $11\{\{1,0,0\},\{0,2,0\},\{0,0,2\}\}$ & \\
\hline 18 Pmmm & $16 \mathrm{mmm}$ & $12\{\{2,0,0\},\{0,1,0\},\{0,0,1\}\}$ & \\
\hline 18 Pmmm & $16 \mathrm{mmm}$ & $13\{\{2,0,0\},\{0,1,0\},\{0,1,2\}\}$ & \\
\hline 18 Pmmm & $16 \mathrm{mmm}$ & $14\{\{2,0,0\},\{0,1,0\},\{0,0,2\}\}$ & \\
\hline 18 Pmmm & $16 \mathrm{mmm}$ & $15\{\{2,0,0\},\{0,2,0\},\{0,0,1\}\}$ & \\
\hline 18 Pmmm & $16 \mathrm{mmm}$ & $16\{\{2,0,0\},\{0,2,0\},\{0,0,2\}\}$ & \\
\hline $19 \mathrm{Cmmm}$ & $2 \overline{1}$ & $1\{\{1,0,0\},\{0,1,0\},\{0,0,1\}\}$ & \\
\hline $19 \mathrm{Cmmm}$ & $2 \overline{1}$ & $2\{\{1,0,0\},\{0,1,0\},\{1,1,2\}\}$ & \\
\hline $19 \mathrm{Cmmm}$ & $2 \overline{1}$ & $3\{\{1,0,0\},\{0,1,0\},\{0,0,2\}\}$ & \\
\hline $19 \mathrm{Cmmm}$ & $2 \overline{1}$ & $4\{\{1,0,0\},\{1,2,0\},\{0,0,1\}\}$ & \\
\hline $19 \mathrm{Cmmm}$ & $2 \overline{1}$ & $5\{\{1,0,0\},\{1,2,0\},\{1,0,2\}\}$ & \\
\hline $19 \mathrm{Cmmm}$ & $2 \overline{1}$ & $6\{\{1,0,0\},\{1,2,0\},\{0,0,2\}\}$ & \\
\hline $19 \mathrm{Cmmm}$ & $2 \overline{1}$ & $7\{\{2,0,0\},\{0,2,0\},\{0,0,1\}\}$ & \\
\hline $19 \mathrm{Cmmm}$ & $2 \overline{1}$ & $8\{\{2,0,0\},\{0,2,0\},\{0,0,2\}\}$ & \\
\hline $19 \mathrm{Cmmm}$ & $32 z$ & $1\left\{\{1,0,0\},\{0,1,0\},\left\{1+c_{1}, 1+c_{1}, 2+2 c_{1}\right\}\right\}$ & \\
\hline $19 \mathrm{Cmmm}$ & $32 z$ & $2\left\{\{1,0,0\},\{0,1,0\},\left\{0,0,1+c_{1}\right\}\right\}$ & \\
\hline $19 \mathrm{Cmmm}$ & $32 z$ & $3\left\{\{1,0,0\},\{1,2,0\},\left\{1+c_{1}, 0,2+2 c_{1}\right\}\right\}$ & \\
\hline $19 \mathrm{Cmmm}$ & $32 z$ & $4\left\{\{1,0,0\},\{1,2,0\},\left\{0,0,1+c_{1}\right\}\right\}$ & \\
\hline $19 \mathrm{Cmmm}$ & $32 z$ & $5\left\{\{2,0,0\},\{0,2,0\},\left\{0,0,1+c_{1}\right\}\right\}$ & \\
\hline $19 \mathrm{Cmmm}$ & $42 y$ & $1\left\{\{1,0,0\},\left\{c_{1}, 1+c_{1}, 0\right\},\{0,0,1\}\right\}$ & \\
\hline $19 \mathrm{Cmmm}$ & $42 y$ & $2\left\{\{1,0,0\},\left\{c_{1}, 1+c_{1}, 0\right\},\{0,0,2\}\right\}$ & \\
\hline $19 \mathrm{Cmmm}$ & $42 y$ & $3\left\{\{1,0,0\},\left\{c_{1}, 1+c_{1}, 0\right\},\{1,1,2\}\right\}$ & \\
\hline $19 \mathrm{Cmmm}$ & $5 m_{x}$ & $1\left\{\{1,0,0\},\left\{c_{1}, 1+c_{1}, 0\right\},\left\{0,0,1+c_{2}\right\}\right\}$ & \\
\hline $19 \mathrm{Cmmm}$ & $5 m_{x}$ & $2\left\{\{1,0,0\},\left\{c_{1}, 1+c_{1}, 0\right\},\left\{1+c_{2}, 1+c_{2}, 2\left(1+c_{2}\right)\right\}\right\}$ & \\
\hline $19 \mathrm{Cmmm}$ & $62 x$ & $1\{\{1,0,0\},\{0,1,0\},\{0,0,1\}\}$ & \\
\hline $19 \mathrm{Cmmm}$ & $62_{x}$ & $2\left\{\{1,0,0\},\left\{1,2+c_{1}, 0\right\},\{0,0,1\}\right\}$ & \\
\hline $19 \mathrm{Cmmm}$ & $62_{x}$ & $3\{\{1,0,0\},\{0,1,0\},\{1,1,2\}\}$ & \\
\hline
\end{tabular}


Table A.1 - continued from previous page

\begin{tabular}{|c|c|c|c|}
\hline ACC & $P_{N}$ & $B_{N}$ matrix row by row & $\mathrm{c} \#$ \\
\hline $19 \mathrm{Cmmm}$ & $62_{x}$ & $4\{\{1,0,0\},\{0,1,0\},\{0,0,2\}\}$ & \\
\hline $19 \mathrm{Cmmm}$ & $62_{x}$ & $5\left\{\{1,0,0\},\left\{1,2+c_{1}, 0\right\},\{0,0,2\}\right\}$ & \\
\hline $19 \mathrm{Cmmm}$ & $62 x$ & $6\left\{\{1,0,0\},\left\{1,2+c_{1}, 0\right\},\{0,1,2\}\right\}$ & \\
\hline $19 \mathrm{Cmmm}$ & $7 m_{y}$ & $1\left\{\{1,0,0\},\{0,1,0\},\left\{0,0,1+c_{1}\right\}\right\}$ & \\
\hline $19 \mathrm{Cmmm}$ & $7 m_{y}$ & $2\left\{\{1,0,0\},\{0,1,0\},\left\{1+c_{1}, 1+c_{1}, 2\left(1+c_{1}\right)\right\}\right\}$ & \\
\hline $19 \mathrm{Cmmm}$ & $7 m_{y}$ & $3\left\{\{1,0,0\},\left\{1,2+c_{1}, 0\right\},\left\{0,0,1+c_{2}\right\}\right\}$ & \\
\hline $19 \mathrm{Cmmm}$ & $7 m_{y}$ & $4\left\{\{1,0,0\},\left\{1,2+c_{1}, 0\right\},\left\{0,1+c_{2}, 2\left(1+c_{2}\right)\right\}\right\}$ & \\
\hline $19 \mathrm{Cmmm}$ & $8 m_{z}$ & $1\left\{\left\{1+c_{1}, 0,0\right\},\left\{0,1+c_{1}, 0\right\},\{0,0,1\}\right\}$ & \\
\hline $19 \mathrm{Cmmm}$ & $8 m_{z}$ & $2\left\{\left\{1+c_{1}, 0,0\right\},\left\{\left(1+c_{1}\right)\left(1+d_{1}\right),\left(1+c_{1}\right)\left(2+d_{1}+d_{2}\right), 0\right\},\{0,0,1\}\right\}$ & 33 \\
\hline $19 \mathrm{Cmmm}$ & $8 m_{z}$ & $3\left\{\left\{1+c_{1}, 0,0\right\},\left\{0,1+c_{1}, 0\right\},\{0,0,2\}\right\}$ & \\
\hline $19 \mathrm{Cmmm}$ & $8 m_{z}$ & $4\left\{\left\{1+c_{1}, 0,0\right\},\left\{\left(1+c_{1}\right)\left(1+d_{1}\right),\left(1+c_{1}\right)\left(2+d_{1}+d_{2}\right), 0\right\},\{0,0,2\}\right\}$ & 33 \\
\hline $19 \mathrm{Cmmm}$ & $8 m_{z}$ & $5\left\{\left\{1+c_{1}, 0,0\right\},\left\{\left(1+c_{1}\right)\left(1+2 c_{2}\right), 2\left(1+c_{1}\right)\left(1+c_{2}\right), 0\right\},\{0,1,2\}\right\}$ & \\
\hline $19 \mathrm{Cmmm}$ & $8 m_{z}$ & $6\left\{\left\{1+c_{1}, 0,0\right\},\left\{\left(1+c_{1}\right)\left(1+2 d_{1}\right),\left(1+c_{1}\right)\left(3+2 d_{1}+d_{2}\right), 0\right\},\{0,1,2\}\right\}$ & 54 \\
\hline $19 \mathrm{Cmmm}$ & $8 m_{z}$ & $7\left\{\left\{1+c_{1}, 0,0\right\},\left\{\left(1+c_{1}\right)\left(1+2 c_{2}\right), 2\left(1+c_{1}\right)\left(1+c_{2}\right), 0\right\},\{1,0,2\}\right\}$ & \\
\hline $19 \mathrm{Cmmm}$ & $8 m_{z}$ & $8\left\{\left\{1+c_{1}, 0,0\right\},\left\{\left(1+c_{1}\right)\left(1+2 d_{1}\right), 2\left(1+c_{1}\right)\left(2+d_{1}+d_{2}\right), 0\right\},\{1,0,2\}\right\}$ & 32 \\
\hline $19 \mathrm{Cmmm}$ & $8 m_{z}$ & $9\left\{\left\{1+c_{1}, 0,0\right\},\left\{\left(1+c_{1}\right) d_{1},\left(1+c_{1}\right)\left(1+d_{1}+2 d_{2}\right), 0\right\},\{1,1,2\}\right\}$ & 66 \\
\hline $19 \mathrm{Cmmm}$ & $92 / m_{z}$ & $1\{\{1,0,0\},\{0,1,0\},\{0,0,1\}\}$ & \\
\hline $19 \mathrm{Cmmm}$ & $92 / m_{z}$ & $2\{\{1,0,0\},\{0,1,0\},\{1,1,2\}\}$ & \\
\hline $19 \mathrm{Cmmm}$ & $92 / m_{z}$ & $3\{\{1,0,0\},\{0,1,0\},\{0,0,2\}\}$ & \\
\hline $19 \mathrm{Cmmm}$ & $92 / m_{z}$ & $4\{\{1,0,0\},\{1,2,0\},\{0,0,1\}\}$ & \\
\hline $19 \mathrm{Cmmm}$ & $92 / m_{z}$ & $5\{\{1,0,0\},\{1,2,0\},\{1,0,2\}\}$ & \\
\hline $19 \mathrm{Cmmm}$ & $92 / m_{z}$ & $6\{\{1,0,0\},\{1,2,0\},\{0,0,2\}\}$ & \\
\hline $19 \mathrm{Cmmm}$ & $92 / m_{z}$ & $7\{\{2,0,0\},\{0,2,0\},\{0,0,1\}\}$ & \\
\hline $19 \mathrm{Cmmm}$ & $92 / m_{z}$ & $8\{\{2,0,0\},\{0,2,0\},\{0,0,2\}\}$ & \\
\hline $19 \mathrm{Cmmm}$ & $102 / m_{y}$ & $1\{\{1,0,0\},\{1,2,0\},\{0,0,1\}\}$ & \\
\hline $19 \mathrm{Cmmm}$ & $102 / m_{y}$ & $2\{\{1,0,0\},\{0,1,0\},\{0,0,1\}\}$ & \\
\hline $19 \mathrm{Cmmm}$ & $102 / m_{y}$ & $3\{\{1,0,0\},\{1,2,0\},\{0,0,2\}\}$ & \\
\hline $19 \mathrm{Cmmm}$ & $102 / m_{y}$ & $4\{\{1,0,0\},\{0,1,0\},\{1,1,2\}\}$ & \\
\hline
\end{tabular}


Table A.1 - continued from previous page

\begin{tabular}{|c|c|c|c|}
\hline ACC & $P_{N}$ & $B_{N}$ matrix row by row & $\mathrm{c \#}$ \\
\hline $19 \mathrm{Cmmm}$ & $102 / m_{y}$ & $5\{\{1,0,0\},\{0,1,0\},\{0,0,2\}\}$ & \\
\hline $19 \mathrm{Cmmm}$ & $112 / m_{x}$ & $1\{\{1,0,0\},\{1,2,0\},\{0,0,1\}\}$ & \\
\hline $19 \mathrm{Cmmm}$ & $112 / m_{x}$ & $2\{\{1,0,0\},\{0,1,0\},\{0,0,1\}\}$ & \\
\hline $19 \mathrm{Cmmm}$ & $112 / m_{x}$ & $3\{\{1,0,0\},\{1,2,0\},\{0,0,2\}\}$ & \\
\hline $19 \mathrm{Cmmm}$ & $112 / m_{x}$ & $4\{\{1,0,0\},\{0,1,0\},\{1,1,2\}\}$ & \\
\hline $19 \mathrm{Cmmm}$ & $112 / m_{x}$ & $5\{\{1,0,0\},\{0,1,0\},\{0,0,2\}\}$ & \\
\hline $19 \mathrm{Cmmm}$ & 12222 & $1\{\{1,0,0\},\{1,2,0\},\{0,0,1\}\}$ & \\
\hline $19 \mathrm{Cmmm}$ & 12222 & $2\{\{1,0,0\},\{0,1,0\},\{0,0,1\}\}$ & \\
\hline $19 \mathrm{Cmmm}$ & 12222 & $3\{\{1,0,0\},\{1,2,0\},\{0,0,2\}\}$ & \\
\hline $19 \mathrm{Cmmm}$ & 12222 & $4\{\{1,0,0\},\{0,1,0\},\{1,1,2\}\}$ & \\
\hline $19 \mathrm{Cmmm}$ & 12222 & $5\{\{1,0,0\},\{0,1,0\},\{0,0,2\}\}$ & \\
\hline $19 \mathrm{Cmmm}$ & $13 m m 2_{z}$ & $1\left\{\{1,0,0\},\{1,2,0\},\left\{0,0,1+c_{1}\right\}\right\}$ & \\
\hline $19 \mathrm{Cmmm}$ & $13 m m 2_{z}$ & $2\left\{\{1,0,0\},\{0,1,0\},\left\{1+c_{1}, 1+c_{1}, 2+2 c_{1}\right\}\right\}$ & \\
\hline $19 \mathrm{Cmmm}$ & $13 m m 2_{z}$ & $3\left\{\{1,0,0\},\{0,1,0\},\left\{0,0,1+c_{1}\right\}\right\}$ & \\
\hline $19 \mathrm{Cmmm}$ & $14 m m 2_{y}$ & $1\left\{\{1,0,0\},\left\{c_{1}, 1+c_{1}, 0\right\},\{0,0,1\}\right\}$ & \\
\hline $19 \mathrm{Cmmm}$ & $14 m m 2_{y}$ & $2\left\{\{1,0,0\},\left\{c_{1}, 1+c_{1}, 0\right\},\{0,0,2\}\right\}$ & \\
\hline $19 \mathrm{Cmmm}$ & $14 m m 2_{y}$ & $3\left\{\{1,0,0\},\left\{c_{1}, 1+c_{1}, 0\right\},\{1,1,2\}\right\}$ & \\
\hline $19 \mathrm{Cmmm}$ & $15 m m 2_{x}$ & $1\{\{1,0,0\},\{0,1,0\},\{0,0,1\}\}$ & \\
\hline $19 \mathrm{Cmmm}$ & $15 m m 2_{x}$ & $2\left\{\{1,0,0\},\left\{1,2+c_{1}, 0\right\},\{0,0,1\}\right\}$ & \\
\hline $19 \mathrm{Cmmm}$ & $15 m m 2_{x}$ & $3\{\{1,0,0\},\{0,1,0\},\{1,1,2\}\}$ & \\
\hline $19 \mathrm{Cmmm}$ & $15 m m 2_{x}$ & $4\{\{1,0,0\},\{0,1,0\},\{0,0,2\}\}$ & \\
\hline $19 \mathrm{Cmmm}$ & $15 m m 2_{x}$ & $5\left\{\{1,0,0\},\left\{1,2+c_{1}, 0\right\},\{0,0,2\}\right\}$ & \\
\hline $19 \mathrm{Cmmm}$ & $15 m m 2_{x}$ & $6\left\{\{1,0,0\},\left\{1,2+c_{1}, 0\right\},\{0,1,2\}\right\}$ & \\
\hline $19 \mathrm{Cmmm}$ & $16 \mathrm{mmm}$ & $1\{\{1,0,0\},\{1,2,0\},\{0,0,1\}\}$ & \\
\hline $19 \mathrm{Cmmm}$ & $16 \mathrm{mmm}$ & $2\{\{1,0,0\},\{0,1,0\},\{0,0,1\}\}$ & \\
\hline $19 \mathrm{Cmmm}$ & $16 \mathrm{mmm}$ & $3\{\{1,0,0\},\{1,2,0\},\{0,0,2\}\}$ & \\
\hline $19 \mathrm{Cmmm}$ & $16 \mathrm{mmm}$ & $4\{\{1,0,0\},\{0,1,0\},\{1,1,2\}\}$ & \\
\hline $19 \mathrm{Cmmm}$ & $16 \mathrm{mmm}$ & $5\{\{1,0,0\},\{0,1,0\},\{0,0,2\}\}$ & \\
\hline
\end{tabular}


Table A.1 - continued from previous page




Table A.1 - continued from previous page

\begin{tabular}{|c|c|c|c|}
\hline ACC & $P_{N}$ & $B_{N}$ matrix row by row & $\mathrm{c \#}$ \\
\hline $20 \mathrm{Fmmm}$ & $7 m_{y}$ & $\begin{aligned} 8 & \left\{\{1,0,0\},\left\{2\left(1+c_{3}+c_{1}\left(2+c_{2}+c_{3}\right)\right), 3+2 c_{3}+2 c_{1}\left(2+c_{2}+c_{3}\right), 0\right\},\right. \\
& \left.\left\{2\left(1+c_{2}\right), 1+2 c_{2}, 2\left(2+c_{2}+c_{3}\right)\right\}\right\}\end{aligned}$ & \\
\hline $20 \mathrm{Fmmm}$ & $7 m_{y}$ & $\begin{aligned} 9 & \left\{\{1,0,0\},\left\{4+c_{2}+3 c_{3}+2 c_{1}\left(2+c_{2}+c_{3}\right),\right.\right. \\
& \left.\left.5+c_{2}+3 c_{3}+2 c_{1}\left(2+c_{2}+c_{3}\right), 0\right\},\left\{2\left(1+c_{2}\right), 1+2 c_{2}, 2\left(2+c_{2}+c_{3}\right)\right\}\right\}\end{aligned}$ & \\
\hline $20 \mathrm{Fmmm}$ & $7 m_{y}$ & $\begin{aligned} 10 & \left\{\{1,0,0\},\left\{1+2 c_{3}+c_{1}\left(3+2 c_{2}+2 c_{3}\right), 2\left(1+c_{3}\right)+c_{1}\left(3+2 c_{2}+2 c_{3}\right), 0\right\}\right. \\
& \left.\left\{2\left(1+c_{2}\right), 1+2 c_{2}, 3+2 c_{2}+2 c_{3}\right\}\right\}\end{aligned}$ & \\
\hline $20 \mathrm{Fmmm}$ & $7 m_{y}$ & $\begin{aligned} 11 & \left\{\{1,0,0\},\left\{2\left(1+c_{3}\right)+c_{1}\left(3+2 c_{2}+2 c_{3}\right), 3+2 c_{3}+c_{1}\left(3+2 c_{2}+2 c_{3}\right), 0\right\}\right. \\
& \left.\left\{1+2 c_{2}, 2 c_{2}, 3+2 c_{2}+2 c_{3}\right\}\right\}\end{aligned}$ & \\
\hline $20 \mathrm{Fmmm}$ & $8 m_{z}$ & $1\left\{\{1,0,0\},\{0,1,0\},\left\{d_{1}, d_{2}, 1+d_{1}+d_{2}\right\}\right\}$ & 3 \\
\hline $20 \mathrm{Fmmm}$ & $8 m_{z}$ & $2\left\{\{1,0,0\},\{1,2,0\},\left\{1+c_{1}, c_{1}, 2+c_{1}\right\}\right\}$ & \\
\hline $20 \mathrm{Fmmm}$ & $8 m_{z}$ & $3\left\{\{1,0,0\},\left\{1,2\left(1+c_{1}\right), 0\right\},\left\{c_{1}, 0,1+c_{1}\right\}\right\}$ & \\
\hline $20 \mathrm{Fmmm}$ & $8 m_{z}$ & $4\left\{\{1,0,0\},\left\{1,2\left(3+d_{1}+d_{2}\right), 0\right\},\left\{5+2 d_{1}+3 d_{2}, 3+d_{1}+d_{2}, 6+2 d_{1}+3 d_{2}\right\}\right\}$ & $25^{*}$ \\
\hline $20 \mathrm{Fmmm}$ & $8 m_{z}$ & $\begin{aligned} 5 & \left\{\{1,0,0\},\left\{1,2\left(2+c_{1}\right), 0\right\}\right. \\
& \left.\left\{5+3 c_{1}+2\left(2+c_{1}\right) d_{1}+2\left(2+c_{1}\right) d_{2}, 2\left(2+c_{1}\right) d_{1},\left(2+c_{1}\right)\left(3+2 d_{1}+2 d_{2}\right)\right\}\right\}\end{aligned}$ & 58 \\
\hline $20 \mathrm{Fmmm}$ & $8 m_{z}$ & $\begin{aligned} 6 & \left\{\{1,0,0\},\left\{1,2\left(2+c_{1}\right), 0\right\},\left\{5+4 d_{1}+2 d_{2}+c_{1}\left(3+2 d_{1}+d_{2}\right)\right.\right. \\
& \left.\left.\left(2+c_{1}\right)\left(1+2 d_{1}\right),\left(2+c_{1}\right)\left(3+2 d_{1}+d_{2}\right)\right\}\right\}\end{aligned}$ & 55 \\
\hline $20 \mathrm{Fmmm}$ & $8 m_{z}$ & $\begin{aligned} 7 & \left\{\{1,0,0\},\left\{1,3+c_{1}, 0\right\}\right. \\
& \left.\left\{2+c_{1}+\left(3+c_{1}\right) d_{1}+\left(3+c_{1}\right) d_{2},\left(3+c_{1}\right) d_{1},\left(3+c_{1}\right)\left(1+d_{1}+d_{2}\right)\right\}\right\}\end{aligned}$ & 30 \\
\hline $20 \mathrm{Fmmm}$ & $8 m_{z}$ & $\begin{aligned} 8 & \left\{\{1,0,0\},\left\{1,2\left(1+d_{1}\right), 0\right\}\right. \\
& \left.\left\{3+3 d_{1}+2\left(1+d_{1}\right) d_{2}+d_{3},\left(1+d_{1}\right)\left(1+2 d_{2}\right), 4+3 d_{1}+2\left(1+d_{1}\right) d_{2}+d_{3}\right\}\right\}\end{aligned}$ & 68 \\
\hline $20 \mathrm{Fmmm}$ & $8 m_{z}$ & $\begin{aligned} 9 & \left\{\{1,0,0\},\left\{1,2+d_{1}, 0\right\}\right. \\
& \left.\left\{2+d_{1}+\left(2+d_{1}\right) d_{2}+d_{3},\left(2+d_{1}\right) d_{2}, 3+d_{1}+\left(2+d_{1}\right) d_{2}+d_{3}\right\}\right\}\end{aligned}$ & 67 \\
\hline $20 \mathrm{Fmmm}$ & $8 m_{z}$ & $\begin{aligned} & 10\left\{\{1,0,0\},\left\{1,2\left(2+d_{1}+d_{2}\right), 0\right\},\left\{4+2 d_{1}+3 d_{2}+2\left(2+d_{1}+d_{2}\right) d_{3},\right.\right. \\
&\left.\left.\left(2+d_{1}+d_{2}\right)\left(1+2 d_{3}\right), 5+2 d_{1}+3 d_{2}+2\left(2+d_{1}+d_{2}\right) d_{3}\right\}\right\}\end{aligned}$ & $72^{*}$ \\
\hline $20 \mathrm{Fmmm}$ & $8 m_{z}$ & $\begin{aligned} 11\left\{\{1,0,0\},\left\{1,2\left(3+d_{1}+d_{2}\right), 0\right\},\left\{6+2 d_{1}+3 d_{2}+2\left(3+d_{1}+d_{2}\right) d_{3},\right.\right. \\
\left.\left.\left(3+d_{1}+d_{2}\right)\left(1+2 d_{3}\right), 7+2 d_{1}+3 d_{2}+2\left(3+d_{1}+d_{2}\right) d_{3}\right\}\right\}\end{aligned}$ & $73^{*}$ \\
\hline $20 \mathrm{Fmmm}$ & $8 m_{z}$ & $\begin{aligned} 12 & \left\{\{1,0,0\},\left\{1,2\left(3+d_{1}+d_{2}\right), 0\right\},\left\{5+2 d_{1}+3 d_{2}+2\left(3+d_{1}+d_{2}\right)\left(1+d_{3}\right),\right.\right. \\
& \left.\left.\left(3+d_{1}+d_{2}\right)\left(3+2 d_{3}\right), 6+2 d_{1}+3 d_{2}+2\left(3+d_{1}+d_{2}\right)\left(1+d_{3}\right)\right\}\right\}\end{aligned}$ & $74^{*}$ \\
\hline
\end{tabular}


Table A.1 - continued from previous page

\begin{tabular}{|c|c|c|c|}
\hline ACC & $P_{N}$ & $B_{N}$ matrix row by row & $\mathrm{c} \#$ \\
\hline $20 \mathrm{Fmmm}$ & $8 m_{z}$ & $\begin{aligned} 13\{ & \{1,0,0\},\left\{1,4+2 d_{1}+d_{2}, 0\right\},\left\{2+d_{1}+d_{2}+\left(4+2 d_{1}+d_{2}\right)\left(1+d_{3}\right),\right. \\
& \left.\left.\left(4+2 d_{1}+d_{2}\right)\left(1+d_{3}\right), 3+d_{1}+d_{2}+\left(4+2 d_{1}+d_{2}\right)\left(1+d_{3}\right)\right\}\right\}\end{aligned}$ & $69^{*}$ \\
\hline $20 \mathrm{Fmmm}$ & $8 m_{z}$ & $\begin{aligned} 14\{ & \{1,0,0\},\left\{1,5+2 d_{1}+d_{2}, 0\right\},\left\{2+d_{1}+d_{2}+\left(5+2 d_{1}+d_{2}\right)\left(1+d_{3}\right)\right. \\
& \left.\left.\left(5+2 d_{1}+d_{2}\right)\left(1+d_{3}\right), 3+d_{1}+d_{2}+\left(5+2 d_{1}+d_{2}\right)\left(1+d_{3}\right)\right\}\right\}\end{aligned}$ & $70^{*}$ \\
\hline $20 \mathrm{Fmmm}$ & $8 m_{z}$ & $\begin{aligned} 15\{ & \{1,0,0\},\left\{1,6+2 d_{1}+d_{2}, 0\right\},\left\{2+d_{1}+d_{2}+\left(6+2 d_{1}+d_{2}\right)\left(1+d_{3}\right),\right. \\
& \left.\left.\left(6+2 d_{1}+d_{2}\right)\left(1+d_{3}\right), 3+d_{1}+d_{2}+\left(6+2 d_{1}+d_{2}\right)\left(1+d_{3}\right)\right\}\right\}\end{aligned}$ & $71^{*}$ \\
\hline 20 Fmmm & $92 / m_{z}$ & $1\{\{1,0,0\},\{0,1,0\},\{0,1,2\}\}$ & \\
\hline $20 \mathrm{Fmmm}$ & $92 / m_{z}$ & $2\{\{1,0,0\},\{0,1,0\},\{1,0,2\}\}$ & \\
\hline $20 \mathrm{Fmmm}$ & $92 / m_{z}$ & $3\{\{1,0,0\},\{0,1,0\},\{0,0,1\}\}$ & \\
\hline 20 Fmmm & $92 / m_{z}$ & $4\{\{1,0,0\},\{1,2,0\},\{1,0,2\}\}$ & \\
\hline $20 \mathrm{Fmmm}$ & $92 / m_{z}$ & $5\{\{1,0,0\},\{1,2,0\},\{0,0,1\}\}$ & \\
\hline 20 Fmmm & $102 / m_{y}$ & $1\{\{1,0,0\},\{0,1,0\},\{0,1,2\}\}$ & \\
\hline $20 \mathrm{Fmmm}$ & $102 / m_{y}$ & $2\{\{1,0,0\},\{0,1,0\},\{1,0,2\}\}$ & \\
\hline $20 \mathrm{Fmmm}$ & $102 / m_{y}$ & $3\{\{1,0,0\},\{0,1,0\},\{0,0,1\}\}$ & \\
\hline 20 Fmmm & $102 / m_{y}$ & $4\{\{1,0,0\},\{1,2,0\},\{1,0,2\}\}$ & \\
\hline $20 \mathrm{Fmmm}$ & $102 / m_{y}$ & $5\{\{1,0,0\},\{1,2,0\},\{0,0,1\}\}$ & \\
\hline $20 \mathrm{Fmmm}$ & $112 / m_{x}$ & $1\{\{1,0,0\},\{0,1,0\},\{0,1,2\}\}$ & \\
\hline $20 \mathrm{Fmmm}$ & $112 / m_{x}$ & $2\{\{1,0,0\},\{0,1,0\},\{1,0,2\}\}$ & \\
\hline $20 \mathrm{Fmmm}$ & $112 / m_{x}$ & $3\{\{1,0,0\},\{0,1,0\},\{0,0,1\}\}$ & \\
\hline $20 \mathrm{Fmmm}$ & $112 / m_{x}$ & $4\{\{1,0,0\},\{1,2,0\},\{1,0,2\}\}$ & \\
\hline $20 \mathrm{Fmmm}$ & $112 / m_{x}$ & $5\{\{1,0,0\},\{1,2,0\},\{0,0,1\}\}$ & \\
\hline $20 \mathrm{Fmmm}$ & 12222 & $1\{\{1,0,0\},\{0,1,0\},\{0,1,2\}\}$ & \\
\hline $20 \mathrm{Fmmm}$ & 12222 & $2\{\{1,0,0\},\{0,1,0\},\{1,0,2\}\}$ & \\
\hline $20 \mathrm{Fmmm}$ & 12222 & $3\{\{1,0,0\},\{0,1,0\},\{0,0,1\}\}$ & \\
\hline 20 Fmmm & 12222 & $4\{\{1,0,0\},\{1,2,0\},\{1,0,2\}\}$ & \\
\hline $20 \mathrm{Fmmm}$ & 12222 & $5\{\{1,0,0\},\{1,2,0\},\{0,0,1\}\}$ & \\
\hline 20 Fmmm & $13 m m 2_{z}$ & $1\left\{\{1,0,0\},\left\{c_{1}, 1+c_{1}, 0\right\},\{1,0,2\}\right\}$ & \\
\hline $20 \mathrm{Fmmm}$ & $13 \mathrm{~mm} 2_{z}$ & $2\left\{\{1,0,0\},\left\{c_{1}, 1+c_{1}, 0\right\},\{0,1,2\}\right\}$ & \\
\hline
\end{tabular}


Table A.1 - continued from previous page

\begin{tabular}{|c|c|c|c|}
\hline ACC & $P_{N}$ & $B_{N}$ matrix row by row & $\mathrm{c} \#$ \\
\hline $20 \mathrm{Fmmm}$ & $13 m m 2_{z}$ & $3\left\{\{1,0,0\},\left\{c_{1}, 1+c_{1}, 0\right\},\{0,0,1\}\right\}$ & \\
\hline $20 \mathrm{Fmmm}$ & $14 m m 2_{y}$ & $1\left\{\{1,0,0\},\{1,2,0\},\left\{c_{1}, 0,1+c_{1}\right\}\right\}$ & \\
\hline $20 \mathrm{Fmmm}$ & $14 m m 2_{y}$ & $2\left\{\{1,0,0\},\{0,1,0\},\left\{c_{1}, 1+c_{1}, 2+2 c_{1}\right\}\right\}$ & \\
\hline $20 \mathrm{Fmmm}$ & $14 m m 2_{y}$ & $3\left\{\{1,0,0\},\{0,1,0\},\left\{c_{1}, 0,1+c_{1}\right\}\right\}$ & \\
\hline $20 \mathrm{Fmmm}$ & $15 m m 2_{x}$ & $1\{\{1,0,0\},\{1,2,0\},\{0,0,1\}\}$ & \\
\hline $20 \mathrm{Fmmm}$ & $15 m m 2_{x}$ & $2\left\{\{1,0,0\},\{1,2,0\},\left\{1+c_{1}, c_{1}, 2+c_{1}\right\}\right\}$ & \\
\hline $20 \mathrm{Fmmm}$ & $15 m m 2_{x}$ & $3\left\{\{1,0,0\},\{0,1,0\},\left\{0, c_{1}, 1+c_{1}\right\}\right\}$ & \\
\hline $20 \mathrm{Fmmm}$ & $15 m m 2_{x}$ & $4\left\{\{1,0,0\},\{0,1,0\},\left\{1+c_{1}, c_{1}, 2+2 c_{1}\right\}\right\}$ & \\
\hline $20 \mathrm{Fmmm}$ & $16 \mathrm{mmm}$ & $1\{\{1,0,0\},\{0,1,0\},\{0,1,2\}\}$ & \\
\hline $20 \mathrm{Fmmm}$ & $16 \mathrm{mmm}$ & $2\{\{1,0,0\},\{0,1,0\},\{1,0,2\}\}$ & \\
\hline $20 \mathrm{Fmmm}$ & $16 \mathrm{mmm}$ & $3\{\{1,0,0\},\{0,1,0\},\{0,0,1\}\}$ & \\
\hline $20 \mathrm{Fmmm}$ & $16 \mathrm{mmm}$ & $4\{\{1,0,0\},\{1,2,0\},\{1,0,2\}\}$ & \\
\hline $20 \mathrm{Fmmm}$ & $16 \mathrm{mmm}$ & $5\{\{1,0,0\},\{1,2,0\},\{0,0,1\}\}$ & \\
\hline $21 \mathrm{Immm}$ & $2 \overline{1}$ & $1\{\{1,0,0\},\{0,1,0\},\{0,0,1\}\}$ & \\
\hline $21 \mathrm{Immm}$ & $2 \overline{1}$ & $2\{\{1,0,0\},\{0,1,0\},\{1,1,2\}\}$ & \\
\hline $21 \mathrm{Immm}$ & $2 \overline{1}$ & $3\{\{1,0,0\},\{1,2,0\},\{0,0,2\}\}$ & \\
\hline $21 \mathrm{Immm}$ & $2 \overline{1}$ & $4\{\{1,0,0\},\{0,2,0\},\{1,0,2\}\}$ & \\
\hline $21 \mathrm{Immm}$ & $2 \overline{1}$ & $5\{\{2,0,0\},\{0,1,0\},\{0,1,2\}\}$ & \\
\hline $21 \mathrm{Immm}$ & $2 \overline{1}$ & $6\{\{2,0,0\},\{0,2,0\},\{0,0,2\}\}$ & \\
\hline $21 \mathrm{Immm}$ & $32 z$ & $1\{\{1,0,0\},\{1,2,0\},\{0,0,2\}\}$ & \\
\hline $21 \mathrm{Immm}$ & $32 z$ & $2\{\{1,0,0\},\{0,1,0\},\{0,0,1\}\}$ & \\
\hline $21 \mathrm{Immm}$ & $32 z$ & $3\left\{\{1,0,0\},\{1,2,0\},\left\{2,2,2\left(2+c_{1}\right)\right\}\right\}$ & \\
\hline $21 \mathrm{Immm}$ & $32 z$ & $4\left\{\{1,0,0\},\{0,1,0\},\left\{3+2 c_{1}, 3+2 c_{1}, 4\left(1+c_{1}\right)\right\}\right\}$ & \\
\hline $21 \mathrm{Immm}$ & $32 z$ & $5\left\{\{1,0,0\},\{0,1,0\},\left\{1,1,2+c_{1}\right\}\right\}$ & \\
\hline $21 \mathrm{Immm}$ & $42 y$ & $1\{\{1,0,0\},\{0,2,0\},\{1,0,2\}\}$ & \\
\hline $21 \mathrm{Immm}$ & $42 y$ & $2\{\{1,0,0\},\{0,1,0\},\{0,0,1\}\}$ & \\
\hline $21 \mathrm{Immm}$ & $42 y$ & $3\left\{\{1,0,0\},\{0,2,0\},\left\{3+2 c_{1}, 2,2\left(2+c_{1}\right)\right\}\right\}$ & \\
\hline 21 Immm & $42 y$ & $4\left\{\{1,0,0\},\{0,1,0\},\left\{3+4 c_{1}, 3+2 c_{1}, 4\left(1+c_{1}\right)\right\}\right\}$ & \\
\hline
\end{tabular}


Table A.1 - continued from previous page

\begin{tabular}{|c|c|c|c|}
\hline ACC & $P_{N}$ & $B_{N}$ matrix row by row & $\mathrm{c} \#$ \\
\hline $21 \mathrm{Immm}$ & $42 y$ & $5\left\{\{1,0,0\},\{0,1,0\},\left\{1+c_{1}, 1,2+c_{1}\right\}\right\}$ & \\
\hline $21 \mathrm{Immm}$ & $5 m_{x}$ & $1\{\{1,0,0\},\{0,1,0\},\{0,0,1\}\}$ & \\
\hline $21 \mathrm{Immm}$ & $5 m_{x}$ & $2\left\{\left\{2+c_{1}, 0,0\right\},\{0,1,0\},\left\{0,1,2+c_{1}\right\}\right\}$ & \\
\hline $21 \mathrm{Immm}$ & $5 m_{x}$ & $3\left\{\left\{2+c_{1}, 0,0\right\},\{0,1,0\},\left\{\left(2+c_{1}\right)\left(1+c_{2}\right), 1,\left(2+c_{1}\right)\left(2+c_{2}\right)\right\}\right\}$ & \\
\hline $21 \mathrm{Immm}$ & $5 m_{x}$ & $4\left\{\left\{1+c_{1}, 0,0\right\},\{0,1,0\},\left\{\left(1+c_{1}\right)\left(1+d_{1}\right), 1,\left(1+c_{1}\right)\left(3+d_{1}+d_{2}\right)\right\}\right\}$ & 38 \\
\hline $21 \mathrm{Immm}$ & $5 m_{x}$ & $5\left\{\left\{1+c_{1}, 0,0\right\},\{0,1,0\},\left\{1+c_{1}, 1,2\left(1+c_{1}\right)\right\}\right\}$ & \\
\hline $21 \mathrm{Immm}$ & $5 m_{x}$ & $6\left\{\left\{1+c_{1}, 0,0\right\},\{0,1,0\},\left\{1+c_{1}, 1,\left(1+c_{1}\right)\left(3+c_{2}\right)\right\}\right\}$ & \\
\hline $21 \mathrm{Immm}$ & $5 m_{x}$ & $7\left\{\left\{1+c_{1}, 0,0\right\},\{0,1,0\},\left\{\left(1+c_{1}\right)\left(2+d_{1}\right), 1,\left(1+c_{1}\right)\left(3+d_{1}+d_{2}\right)\right\}\right\}$ & 42 \\
\hline $21 \mathrm{Immm}$ & $62_{x}$ & $1\{\{1,0,0\},\{0,1,0\},\{0,0,1\}\}$ & \\
\hline $21 \mathrm{Immm}$ & $62_{x}$ & $2\left\{\{1,0,0\},\{0,1,0\},\left\{3+2 c_{1}, 3+4 c_{1}, 4\left(1+c_{1}\right)\right\}\right\}$ & \\
\hline $21 \mathrm{Immm}$ & $62_{x}$ & $3\left\{\{1,0,0\},\{0,1,0\},\left\{1,1+c_{1}, 2+c_{1}\right\}\right\}$ & \\
\hline $21 \mathrm{Immm}$ & $62_{x}$ & $4\{\{2,0,0\},\{0,1,0\},\{0,1,2\}\}$ & \\
\hline $21 \mathrm{Immm}$ & $62 x$ & $5\left\{\{2,0,0\},\{0,1,0\},\left\{2,3+2 c_{1}, 2\left(2+c_{1}\right)\right\}\right\}$ & \\
\hline $21 \mathrm{Immm}$ & $7 m_{y}$ & $1\{\{1,0,0\},\{0,1,0\},\{0,0,1\}\}$ & \\
\hline $21 \mathrm{Immm}$ & $7 m_{y}$ & $2\{\{1,0,0\},\{0,1,0\},\{1,1,3\}\}$ & \\
\hline $21 \mathrm{Immm}$ & $7 m_{y}$ & $3\{\{1,0,0\},\{0,2,0\},\{1,2,8\}\}$ & \\
\hline $21 \mathrm{Immm}$ & $7 m_{y}$ & $4\left\{\{1,0,0\},\{0,1,0\},\left\{1,1,4+c_{1}\right\}\right\}$ & \\
\hline $21 \mathrm{Immm}$ & $7 m_{y}$ & $5\left\{\{1,0,0\},\left\{0,2+c_{1}, 0\right\},\left\{1,0,2+c_{1}\right\}\right\}$ & \\
\hline $21 \mathrm{Immm}$ & $7 m_{y}$ & $6\left\{\{1,0,0\},\left\{0,2+c_{1}, 0\right\},\left\{1,2+c_{1}, 3\left(2+c_{1}\right)\right\}\right\}$ & \\
\hline $21 \mathrm{Immm}$ & $7 m_{y}$ & $7\left\{\{1,0,0\},\left\{0,3+c_{1}, 0\right\},\left\{1,3+c_{1}, 4\left(3+c_{1}\right)\right\}\right\}$ & \\
\hline $21 \mathrm{Immm}$ & $7 m_{y}$ & $8\left\{\{1,0,0\},\left\{0,2+c_{1}, 0\right\},\left\{1,\left(2+c_{1}\right)\left(1+c_{2}\right),\left(2+c_{1}\right)\left(2+c_{2}\right)\right\}\right\}$ & \\
\hline $21 \mathrm{Immm}$ & $7 m_{y}$ & $9\left\{\{1,0,0\},\{0,1,0\},\left\{1,2+d_{1}, 5+d_{1}+d_{2}\right\}\right\}$ & 17 \\
\hline $21 \mathrm{Immm}$ & $7 m_{y}$ & $10\left\{\{1,0,0\},\{0,2,0\},\left\{1,2\left(1+d_{1}\right), 2\left(5+d_{1}+d_{2}\right)\right\}\right\}$ & 16 \\
\hline $21 \mathrm{Immm}$ & $7 m_{y}$ & $11\left\{\{1,0,0\},\{0,2,0\},\left\{1,2\left(2+d_{1}\right), 2\left(5+d_{1}+d_{2}\right)\right\}\right\}$ & 17 \\
\hline $21 \mathrm{Immm}$ & $7 m_{y}$ & $12\left\{\{1,0,0\},\left\{0,3+c_{1}, 0\right\},\left\{1,\left(3+c_{1}\right)\left(1+d_{1}\right),\left(3+c_{1}\right)\left(5+d_{1}+d_{2}\right)\right\}\right\}$ & 49 \\
\hline $21 \mathrm{Immm}$ & $7 m_{y}$ & $13\left\{\{1,0,0\},\left\{0,3+c_{1}, 0\right\},\left\{1,\left(3+c_{1}\right)\left(2+d_{1}\right),\left(3+c_{1}\right)\left(5+d_{1}+d_{2}\right)\right\}\right\}$ & 50 \\
\hline $21 \mathrm{Immm}$ & $7 m_{y}$ & $14\left\{\{1,0,0\},\{0,1,0\},\left\{1,2+c_{1}, 3+c_{1}\right\}\right\}$ & \\
\hline $21 \mathrm{Immm}$ & $7 m_{y}$ & $15\left\{\{1,0,0\},\left\{0,1+c_{1}, 0\right\},\left\{1,1+c_{1}, 2\left(1+c_{1}\right)\right\}\right\}$ & \\
\hline
\end{tabular}


Table A.1 - continued from previous page

\begin{tabular}{|c|c|c|c|}
\hline ACC & $P_{N}$ & $B_{N}$ matrix row by row & $\mathrm{c} \#$ \\
\hline $21 \mathrm{Immm}$ & $7 m_{y}$ & $16\left\{\{1,0,0\},\left\{0,1+c_{1}, 0\right\},\left\{1,1+c_{1},\left(1+c_{1}\right)\left(3+c_{2}\right)\right\}\right\}$ & \\
\hline $21 \mathrm{Immm}$ & $7 m_{y}$ & $17\left\{\{1,0,0\},\left\{0,2+c_{1}, 0\right\},\left\{1,\left(2+c_{1}\right)\left(2+c_{2}\right),\left(2+c_{1}\right)\left(3+c_{2}\right)\right\}\right\}$ & \\
\hline $21 \mathrm{Immm}$ & $7 m_{y}$ & $18\left\{\{1,0,0\},\{0,1,0\},\left\{1,3+d_{1}, 5+d_{1}+d_{2}\right\}\right\}$ & 18 \\
\hline $21 \mathrm{Immm}$ & $7 m_{y}$ & $19\left\{\{1,0,0\},\left\{0,2+c_{1}, 0\right\},\left\{1,\left(2+c_{1}\right)\left(2+d_{1}\right),\left(2+c_{1}\right)\left(5+d_{1}+d_{2}\right)\right\}\right\}$ & 51 \\
\hline $21 \mathrm{Immm}$ & $7 m_{y}$ & $20\left\{\{1,0,0\},\left\{0,2+c_{1}, 0\right\},\left\{1,\left(2+c_{1}\right)\left(3+d_{1}\right),\left(2+c_{1}\right)\left(5+d_{1}+d_{2}\right)\right\}\right\}$ & 52 \\
\hline $21 \mathrm{Immm}$ & $8 m_{z}$ & $1\{\{1,0,0\},\{0,1,0\},\{0,0,1\}\}$ & \\
\hline $21 \mathrm{Immm}$ & $8 m_{z}$ & $2\left\{\{1,0,0\},\{0,1,0\},\left\{1+d_{1}, 1+d_{2}, 2+d_{1}+d_{2}\right\}\right\}$ & 4 \\
\hline $21 \mathrm{Immm}$ & $8 m_{z}$ & $3\{\{1,0,0\},\{1,2,0\},\{0,0,2\}\}$ & \\
\hline $21 \mathrm{Immm}$ & $8 m_{z}$ & $4\{\{1,0,0\},\{1,3,0\},\{0,3,6\}\}$ & \\
\hline $21 \mathrm{Immm}$ & $8 m_{z}$ & $5\left\{\{1,0,0\},\left\{1,2+c_{1}, 0\right\},\left\{0,0,2+c_{1}\right\}\right\}$ & \\
\hline $21 \mathrm{Immm}$ & $8 m_{z}$ & $6\left\{\{1,0,0\},\{1,2,0\},\left\{0,2,2\left(2+c_{1}\right)\right\}\right\}$ & \\
\hline $21 \mathrm{Immm}$ & $8 m_{z}$ & $7\left\{\{1,0,0\},\left\{1,4+c_{1}, 0\right\},\left\{0,4+c_{1}, 2\left(4+c_{1}\right)\right\}\right\}$ & \\
\hline $21 \mathrm{Immm}$ & $8 m_{z}$ & $8\left\{\{1,0,0\},\{1,2,0\},\left\{0,2\left(2+d_{1}\right), 2\left(3+d_{1}+d_{2}\right)\right\}\right\}$ & 9 \\
\hline $21 \mathrm{Immm}$ & $8 m_{z}$ & $9\left\{\{1,0,0\},\{1,3,0\},\left\{0,3\left(1+d_{1}\right), 3\left(3+d_{1}+d_{2}\right)\right\}\right\}$ & 6 \\
\hline $21 \mathrm{Immm}$ & $8 m_{z}$ & $10\left\{\{1,0,0\},\{1,3,0\},\left\{0,3\left(2+d_{1}\right), 3\left(3+d_{1}+d_{2}\right)\right\}\right\}$ & 9 \\
\hline $21 \mathrm{Immm}$ & $8 m_{z}$ & $11\left\{\{1,0,0\},\left\{1,4+c_{1}, 0\right\},\left\{0,\left(4+c_{1}\right)\left(1+d_{1}\right),\left(4+c_{1}\right)\left(3+d_{1}+d_{2}\right)\right\}\right\}$ & 38 \\
\hline $21 \mathrm{Immm}$ & $8 m_{z}$ & $12\left\{\{1,0,0\},\left\{1,4+c_{1}, 0\right\},\left\{0,\left(4+c_{1}\right)\left(2+d_{1}\right),\left(4+c_{1}\right)\left(3+d_{1}+d_{2}\right)\right\}\right\}$ & 42 \\
\hline $21 \mathrm{Immm}$ & $92 / m_{z}$ & $1\{\{1,0,0\},\{0,1,0\},\{0,0,1\}\}$ & \\
\hline $21 \mathrm{Immm}$ & $92 / m_{z}$ & $2\{\{1,0,0\},\{1,2,0\},\{0,0,2\}\}$ & \\
\hline $21 \mathrm{Immm}$ & $92 / m_{z}$ & $3\{\{1,0,0\},\{0,1,0\},\{1,1,2\}\}$ & \\
\hline $21 \mathrm{Immm}$ & $102 / m_{y}$ & $1\{\{1,0,0\},\{0,1,0\},\{1,1,2\}\}$ & \\
\hline $21 \mathrm{Immm}$ & $102 / m_{y}$ & $2\{\{1,0,0\},\{0,1,0\},\{0,0,1\}\}$ & \\
\hline $21 \mathrm{Immm}$ & $102 / m_{y}$ & $3\{\{1,0,0\},\{0,2,0\},\{1,0,2\}\}$ & \\
\hline $21 \mathrm{Immm}$ & $112 / m_{x}$ & $1\{\{1,0,0\},\{0,1,0\},\{1,1,2\}\}$ & \\
\hline $21 \mathrm{Immm}$ & $112 / m_{x}$ & $2\{\{1,0,0\},\{0,1,0\},\{0,0,1\}\}$ & \\
\hline $21 \mathrm{Immm}$ & $112 / m_{x}$ & $3\{\{2,0,0\},\{0,1,0\},\{0,1,2\}\}$ & \\
\hline $21 \mathrm{Immm}$ & 12222 & $1\{\{1,0,0\},\{0,1,0\},\{3,3,4\}\}$ & \\
\hline $21 \mathrm{Immm}$ & 12222 & $2\{\{1,0,0\},\{0,1,0\},\{1,1,2\}\}$ & \\
\hline
\end{tabular}


Table A.1 - continued from previous page

\begin{tabular}{|c|c|c|c|}
\hline ACC & $P_{N}$ & $B_{N}$ matrix row by row & $\mathrm{c} \#$ \\
\hline $21 \mathrm{Immm}$ & 12222 & $3\{\{1,0,0\},\{0,1,0\},\{0,0,1\}\}$ & \\
\hline $21 \mathrm{Immm}$ & 12222 & $4\{\{1,0,0\},\{0,1,0\},\{3,3,4\}\}$ & \\
\hline $21 \mathrm{Immm}$ & 12222 & $5\{\{1,0,0\},\{0,1,0\},\{1,1,2\}\}$ & \\
\hline $21 \mathrm{Immm}$ & 12222 & $6\{\{1,0,0\},\{0,1,0\},\{0,0,1\}\}$ & \\
\hline $21 \mathrm{Immm}$ & $13 m m 2_{z}$ & $1\{\{1,0,0\},\{0,1,0\},\{0,0,1\}\}$ & \\
\hline $21 \mathrm{Immm}$ & $13 m m 2_{z}$ & $2\left\{\{1,0,0\},\{0,1,0\},\left\{1,1,2+c_{1}\right\}\right\}$ & \\
\hline $21 \mathrm{Immm}$ & $14 m m 2_{y}$ & $1\{\{1,0,0\},\{0,1,0\},\{0,0,1\}\}$ & \\
\hline $21 \mathrm{Immm}$ & $14 m m 2_{y}$ & $2\left\{\{1,0,0\},\{0,1,0\},\left\{1+c_{1}, 1,2+c_{1}\right\}\right\}$ & \\
\hline $21 \mathrm{Immm}$ & $15 m m 2_{x}$ & $1\{\{1,0,0\},\{0,1,0\},\{0,0,1\}\}$ & \\
\hline $21 \mathrm{Immm}$ & $15 m m 2_{x}$ & $2\left\{\{1,0,0\},\{0,1,0\},\left\{1,1+c_{1}, 2+c_{1}\right\}\right\}$ & \\
\hline $21 \mathrm{Immm}$ & $16 \mathrm{mmm}$ & $1\{\{1,0,0\},\{0,1,0\},\{1,1,2\}\}$ & \\
\hline $21 \mathrm{Immm}$ & $16 \mathrm{mmm}$ & $2\{\{1,0,0\},\{0,1,0\},\{0,0,1\}\}$ & \\
\hline $22 P 4$ & $22_{z}$ & $1\left\{\{1,0,0\},\{0,1,0\},\left\{1+c_{1}, 1+c_{1}, 2+2 c_{1}\right\}\right\}$ & \\
\hline $22 P 4$ & $22_{z}$ & $2\left\{\{1,0,0\},\{0,1,0\},\left\{0,0,1+c_{1}\right\}\right\}$ & \\
\hline $22 P 4$ & $22 z$ & $3\left\{\{1,0,0\},\{1,2,0\},\left\{1+c_{1}, 0,2+2 c_{1}\right\}\right\}$ & \\
\hline $22 P 4$ & $22 z$ & $4\left\{\{1,0,0\},\{1,2,0\},\left\{0,0,1+c_{1}\right\}\right\}$ & \\
\hline $22 P 4$ & $22_{z}$ & $5\left\{\{2,0,0\},\{0,2,0\},\left\{0,0,1+c_{1}\right\}\right\}$ & \\
\hline $22 P 4$ & 34 & $1\left\{\{1,0,0\},\{1,2,0\},\left\{0,0,1+c_{1}\right\}\right\}$ & \\
\hline $22 P 4$ & 34 & $2\left\{\{1,0,0\},\{0,1,0\},\left\{1+c_{1}, 1+c_{1}, 2+2 c_{1}\right\}\right\}$ & \\
\hline $22 P 4$ & 34 & $3\left\{\{1,0,0\},\{0,1,0\},\left\{0,0,1+c_{1}\right\}\right\}$ & \\
\hline $23 I 4$ & $22_{z}$ & $1\{\{1,0,0\},\{1,2,0\},\{0,0,2\}\}$ & \\
\hline $23 I 4$ & $22_{z}$ & $2\{\{1,0,0\},\{0,1,0\},\{0,0,1\}\}$ & \\
\hline $23 I 4$ & $22_{z}$ & $3\left\{\{1,0,0\},\{1,2,0\},\left\{2,2,2\left(2+c_{1}\right)\right\}\right\}$ & \\
\hline $23 I 4$ & $22_{z}$ & $4\left\{\{1,0,0\},\{0,1,0\},\left\{3+2 c_{1}, 3+2 c_{1}, 4\left(1+c_{1}\right)\right\}\right\}$ & \\
\hline $23 I 4$ & $22 z$ & $5\left\{\{1,0,0\},\{0,1,0\},\left\{1,1,2+c_{1}\right\}\right\}$ & \\
\hline $23 I 4$ & 34 & $1\{\{1,0,0\},\{0,1,0\},\{0,0,1\}\}$ & \\
\hline $23 I 4$ & 34 & $2\left\{\{1,0,0\},\{0,1,0\},\left\{1,1,2+c_{1}\right\}\right\}$ & \\
\hline $24 P \overline{4}$ & $22_{z}$ & $1\left\{\{1,0,0\},\{0,1,0\},\left\{1+c_{1}, 1+c_{1}, 2+2 c_{1}\right\}\right\}$ & \\
\hline
\end{tabular}


Table A.1 - continued from previous page

\begin{tabular}{|c|c|c|c|}
\hline ACC & $P_{N}$ & $B_{N}$ matrix row by row & $\mathrm{c \#}$ \\
\hline $24 P \overline{4}$ & $22_{z}$ & $2\left\{\{1,0,0\},\{0,1,0\},\left\{0,0,1+c_{1}\right\}\right\}$ & \\
\hline $24 P \overline{4}$ & $22 z$ & $3\left\{\{1,0,0\},\{1,2,0\},\left\{1+c_{1}, 0,2+2 c_{1}\right\}\right\}$ & \\
\hline $24 P \overline{4}$ & $22 z$ & $4\left\{\{1,0,0\},\{1,2,0\},\left\{0,0,1+c_{1}\right\}\right\}$ & \\
\hline $24 P \overline{4}$ & $22 z$ & $5\left\{\{2,0,0\},\{0,2,0\},\left\{0,0,1+c_{1}\right\}\right\}$ & \\
\hline $24 P \overline{4}$ & $3 \overline{4}$ & $1\{\{1,0,0\},\{1,2,0\},\{0,0,1\}\}$ & \\
\hline $24 P \overline{4}$ & $3 \overline{4}$ & $2\{\{1,0,0\},\{0,1,0\},\{0,0,1\}\}$ & \\
\hline $24 P \overline{4}$ & $3 \overline{4}$ & $3\{\{1,0,0\},\{1,2,0\},\{0,0,2\}\}$ & \\
\hline $24 P \overline{4}$ & $3 \overline{4}$ & $4\{\{1,0,0\},\{0,1,0\},\{1,1,2\}\}$ & \\
\hline $24 P \overline{4}$ & $3 \overline{4}$ & $5\{\{1,0,0\},\{0,1,0\},\{0,0,2\}\}$ & \\
\hline $25 I \overline{4}$ & $22 z$ & $1\{\{1,0,0\},\{1,2,0\},\{0,0,2\}\}$ & \\
\hline $25 I \overline{4}$ & $22_{z}$ & $2\{\{1,0,0\},\{0,1,0\},\{0,0,1\}\}$ & \\
\hline $25 I \overline{4}$ & $22 z$ & $3\left\{\{1,0,0\},\{1,2,0\},\left\{2,2,2\left(2+c_{1}\right)\right\}\right\}$ & \\
\hline $25 I \overline{4}$ & $22 z$ & $4\left\{\{1,0,0\},\{0,1,0\},\left\{3+2 c_{1}, 3+2 c_{1}, 4\left(1+c_{1}\right)\right\}\right\}$ & \\
\hline $25 I \overline{4}$ & $22 z$ & $5\left\{\{1,0,0\},\{0,1,0\},\left\{1,1,2+c_{1}\right\}\right\}$ & \\
\hline $25 I \overline{4}$ & $3 \overline{4}$ & $1\{\{1,0,0\},\{0,1,0\},\{3,3,4\}\}$ & \\
\hline $25 I \overline{4}$ & $3 \overline{4}$ & $2\{\{1,0,0\},\{0,1,0\},\{1,1,2\}\}$ & \\
\hline $25 I \overline{4}$ & $3 \overline{4}$ & $3\{\{1,0,0\},\{0,1,0\},\{0,0,1\}\}$ & \\
\hline $25 I \overline{4}$ & $3 \overline{4}$ & $4\{\{1,0,0\},\{0,1,0\},\{3,3,4\}\}$ & \\
\hline $25 I \overline{4}$ & $3 \overline{4}$ & $5\{\{1,0,0\},\{0,1,0\},\{1,1,2\}\}$ & \\
\hline $25 I \overline{4}$ & $3 \overline{4}$ & $6\{\{1,0,0\},\{0,1,0\},\{0,0,1\}\}$ & \\
\hline $26 P 4 / m$ & $2 \overline{1}$ & $1\{\{1,0,0\},\{0,1,0\},\{0,0,1\}\}$ & \\
\hline $26 P 4 / m$ & $2 \overline{1}$ & $2\{\{1,0,0\},\{0,1,0\},\{1,1,2\}\}$ & \\
\hline $26 P 4 / m$ & $2 \overline{1}$ & $3\{\{1,0,0\},\{0,1,0\},\{0,0,2\}\}$ & \\
\hline $26 P 4 / m$ & $2 \overline{1}$ & $4\{\{1,0,0\},\{1,2,0\},\{0,0,1\}\}$ & \\
\hline $26 P 4 / m$ & $2 \overline{1}$ & $5\{\{1,0,0\},\{1,2,0\},\{1,0,2\}\}$ & \\
\hline $26 P 4 / m$ & $2 \overline{1}$ & $6\{\{1,0,0\},\{1,2,0\},\{0,0,2\}\}$ & \\
\hline $26 P 4 / m$ & $2 \overline{1}$ & $7\{\{2,0,0\},\{0,2,0\},\{0,0,1\}\}$ & \\
\hline $26 P 4 / m$ & $2 \overline{1}$ & $8\{\{2,0,0\},\{0,2,0\},\{0,0,2\}\}$ & \\
\hline
\end{tabular}


Table A.1 - continued from previous page

\begin{tabular}{|c|c|c|c|}
\hline ACC & $P_{N}$ & $B_{N}$ matrix row by row & $\mathrm{c} \#$ \\
\hline $26 P 4 / m$ & $32 z$ & $1\left\{\{1,0,0\},\{0,1,0\},\left\{1+c_{1}, 1+c_{1}, 2+2 c_{1}\right\}\right\}$ & \\
\hline $26 P 4 / m$ & $32 z$ & $2\left\{\{1,0,0\},\{0,1,0\},\left\{0,0,1+c_{1}\right\}\right\}$ & \\
\hline $26 P 4 / m$ & $32 z$ & $3\left\{\{1,0,0\},\{1,2,0\},\left\{1+c_{1}, 0,2+2 c_{1}\right\}\right\}$ & \\
\hline $26 P 4 / m$ & $32 z$ & $4\left\{\{1,0,0\},\{1,2,0\},\left\{0,0,1+c_{1}\right\}\right\}$ & \\
\hline $26 P 4 / m$ & $32 z$ & $5\left\{\{2,0,0\},\{0,2,0\},\left\{0,0,1+c_{1}\right\}\right\}$ & \\
\hline $26 P 4 / m$ & $4 m_{z}$ & $1\left\{\left\{1+c_{1}, 0,0\right\},\left\{0,1+c_{1}, 0\right\},\{0,0,1\}\right\}$ & \\
\hline $26 P 4 / m$ & $4 m_{z}$ & $2\left\{\left\{1+c_{1}, 0,0\right\},\left\{\left(1+c_{1}\right)\left(1+d_{1}\right),\left(1+c_{1}\right)\left(2+d_{1}+d_{2}\right), 0\right\},\{0,0,1\}\right\}$ & 35 \\
\hline $26 P 4 / m$ & $4 m_{z}$ & $3\left\{\left\{1+c_{1}, 0,0\right\},\left\{0,1+c_{1}, 0\right\},\{0,0,2\}\right\}$ & \\
\hline $26 P 4 / m$ & $4 m_{z}$ & $4\left\{\left\{1+c_{1}, 0,0\right\},\left\{\left(1+c_{1}\right)\left(1+d_{1}\right),\left(1+c_{1}\right)\left(2+d_{1}+d_{2}\right), 0\right\},\{0,0,2\}\right\}$ & 35 \\
\hline $26 P 4 / m$ & $4 m_{z}$ & $5\left\{\left\{1+c_{1}, 0,0\right\},\left\{\left(1+c_{1}\right)\left(1+2 d_{1}\right),\left(1+c_{1}\right)\left(2+2 d_{1}+d_{2}\right), 0\right\},\{0,1,2\}\right\}$ & 53 \\
\hline $26 P 4 / m$ & $4 m_{z}$ & $6\left\{\left\{1+c_{1}, 0,0\right\},\left\{\left(1+c_{1}\right)\left(1+2 d_{1}\right), 2\left(1+c_{1}\right)\left(1+d_{1}+d_{2}\right), 0\right\},\{1,0,2\}\right\}$ & 31 \\
\hline $26 P 4 / m$ & $4 m_{z}$ & $7\left\{\left\{1+c_{1}, 0,0\right\},\left\{0,1+c_{1}, 0\right\},\{1,1,2\}\right\}$ & \\
\hline $26 P 4 / m$ & $4 m_{z}$ & $8\left\{\left\{1+c_{1}, 0,0\right\},\left\{\left(1+c_{1}\right) d_{1},\left(1+c_{1}\right)\left(3+d_{1}+2 d_{2}\right), 0\right\},\{1,1,2\}\right\}$ & 64 \\
\hline $26 P 4 / m$ & $52 / m_{z}$ & $1\{\{1,0,0\},\{0,1,0\},\{0,0,1\}\}$ & \\
\hline $26 P 4 / m$ & $52 / m_{z}$ & $2\{\{1,0,0\},\{0,1,0\},\{1,1,2\}\}$ & \\
\hline $26 P 4 / m$ & $52 / m_{z}$ & $3\{\{1,0,0\},\{0,1,0\},\{0,0,2\}\}$ & \\
\hline $26 P 4 / m$ & $52 / m_{z}$ & $4\{\{1,0,0\},\{1,2,0\},\{0,0,1\}\}$ & \\
\hline $26 P 4 / m$ & $52 / m_{z}$ & $5\{\{1,0,0\},\{1,2,0\},\{1,0,2\}\}$ & \\
\hline $26 P 4 / m$ & $52 / m_{z}$ & $6\{\{1,0,0\},\{1,2,0\},\{0,0,2\}\}$ & \\
\hline $26 P 4 / m$ & $52 / m_{z}$ & $7\{\{2,0,0\},\{0,2,0\},\{0,0,1\}\}$ & \\
\hline $26 P 4 / m$ & $52 / m_{z}$ & $8\{\{2,0,0\},\{0,2,0\},\{0,0,2\}\}$ & \\
\hline $26 P 4 / m$ & $6 \overline{4}$ & $1\{\{1,0,0\},\{1,2,0\},\{0,0,1\}\}$ & \\
\hline $26 P 4 / m$ & $6 \overline{4}$ & $2\{\{1,0,0\},\{0,1,0\},\{0,0,1\}\}$ & \\
\hline $26 P 4 / m$ & $6 \overline{4}$ & $3\{\{1,0,0\},\{1,2,0\},\{0,0,2\}\}$ & \\
\hline $26 P 4 / m$ & $6 \overline{4}$ & $4\{\{1,0,0\},\{0,1,0\},\{1,1,2\}\}$ & \\
\hline $26 P 4 / m$ & $6 \overline{4}$ & $5\{\{1,0,0\},\{0,1,0\},\{0,0,2\}\}$ & \\
\hline $26 P 4 / m$ & 74 & $1\left\{\{1,0,0\},\{1,2,0\},\left\{0,0,1+c_{1}\right\}\right\}$ & \\
\hline $26 P 4 / m$ & 74 & $2\left\{\{1,0,0\},\{0,1,0\},\left\{1+c_{1}, 1+c_{1}, 2+2 c_{1}\right\}\right\}$ & \\
\hline
\end{tabular}


Table A.1 - continued from previous page

\begin{tabular}{|c|c|c|c|}
\hline ACC & $P_{N}$ & $B_{N}$ matrix row by row & $\mathrm{c} \#$ \\
\hline $26 P 4 / m$ & 74 & $3\left\{\{1,0,0\},\{0,1,0\},\left\{0,0,1+c_{1}\right\}\right\}$ & \\
\hline $26 P 4 / m$ & $84 / m$ & $1\{\{1,0,0\},\{1,2,0\},\{0,0,1\}\}$ & \\
\hline $26 P 4 / m$ & $84 / m$ & $2\{\{1,0,0\},\{0,1,0\},\{0,0,1\}\}$ & \\
\hline $26 P 4 / m$ & $84 / m$ & $3\{\{1,0,0\},\{1,2,0\},\{0,0,2\}\}$ & \\
\hline $26 P 4 / m$ & $84 / m$ & $4\{\{1,0,0\},\{0,1,0\},\{1,1,2\}\}$ & \\
\hline $26 P 4 / m$ & $84 / m$ & $5\{\{1,0,0\},\{0,1,0\},\{0,0,2\}\}$ & \\
\hline $27 I 4 / m$ & $2 \overline{1}$ & $1\{\{1,0,0\},\{0,1,0\},\{0,0,1\}\}$ & \\
\hline $27 I 4 / m$ & $2 \overline{1}$ & $2\{\{1,0,0\},\{0,1,0\},\{1,1,2\}\}$ & \\
\hline $27 I 4 / m$ & $2 \overline{1}$ & $3\{\{1,0,0\},\{1,2,0\},\{0,0,2\}\}$ & \\
\hline $27 I 4 / m$ & $2 \overline{1}$ & $4\{\{2,0,0\},\{0,2,0\},\{0,0,2\}\}$ & \\
\hline $27 I 4 / m$ & $32 z$ & $1\{\{1,0,0\},\{1,2,0\},\{0,0,2\}\}$ & \\
\hline $27 I 4 / m$ & $32 z$ & $2\{\{1,0,0\},\{0,1,0\},\{0,0,1\}\}$ & \\
\hline $27 I 4 / m$ & $32 z$ & $3\left\{\{1,0,0\},\{1,2,0\},\left\{2,2,2\left(2+c_{1}\right)\right\}\right\}$ & \\
\hline $27 I 4 / m$ & $32 z$ & $4\left\{\{1,0,0\},\{0,1,0\},\left\{3+2 c_{1}, 3+2 c_{1}, 4\left(1+c_{1}\right)\right\}\right\}$ & \\
\hline $27 I 4 / m$ & $32 z$ & $5\left\{\{1,0,0\},\{0,1,0\},\left\{1,1,2+c_{1}\right\}\right\}$ & \\
\hline $27 I 4 / m$ & $4 m_{z}$ & $1\{\{1,0,0\},\{0,1,0\},\{0,0,1\}\}$ & \\
\hline $27 I 4 / m$ & $4 m_{z}$ & $2\left\{\{1,0,0\},\{0,1,0\},\left\{1+d_{1}, 1+d_{2}, 2+d_{1}+d_{2}\right\}\right\}$ & 5 \\
\hline $27 I 4 / m$ & $4 m_{z}$ & $3\{\{1,0,0\},\{1,2,0\},\{0,0,2\}\}$ & \\
\hline $27 I 4 / m$ & $4 m_{z}$ & $4\{\{1,0,0\},\{1,2,0\},\{0,2,4\}\}$ & \\
\hline $27 I 4 / m$ & $4 m_{z}$ & $5\{\{1,0,0\},\{1,3,0\},\{0,3,6\}\}$ & \\
\hline $27 I 4 / m$ & $4 m_{z}$ & $6\left\{\{1,0,0\},\left\{1,2+c_{1}, 0\right\},\left\{0,0,2+c_{1}\right\}\right\}$ & \\
\hline $27 I 4 / m$ & $4 m_{z}$ & $7\left\{\{1,0,0\},\left\{1,4+c_{1}, 0\right\},\left\{0,4+c_{1}, 2\left(4+c_{1}\right)\right\}\right\}$ & \\
\hline $27 I 4 / m$ & $4 m_{z}$ & $8\left\{\{1,0,0\},\{1,2,0\},\left\{0,2\left(2+d_{1}\right), 2\left(3+d_{1}+d_{2}\right)\right\}\right\}$ & 10 \\
\hline $27 I 4 / m$ & $4 m_{z}$ & $9\left\{\{1,0,0\},\{1,3,0\},\left\{0,3\left(1+d_{1}\right), 3\left(3+d_{1}+d_{2}\right)\right\}\right\}$ & 7 \\
\hline $27 I 4 / m$ & $4 m_{z}$ & $10\left\{\{1,0,0\},\{1,3,0\},\left\{0,3\left(2+d_{1}\right), 3\left(3+d_{1}+d_{2}\right)\right\}\right\}$ & 10 \\
\hline $27 I 4 / m$ & $4 m_{z}$ & $11\left\{\{1,0,0\},\left\{1,4+c_{1}, 0\right\},\left\{0,\left(4+c_{1}\right)\left(1+d_{1}\right),\left(4+c_{1}\right)\left(3+d_{1}+d_{2}\right)\right\}\right\}$ & 39 \\
\hline $27 I 4 / m$ & $4 m_{z}$ & $12\left\{\{1,0,0\},\left\{1,4+c_{1}, 0\right\},\left\{0,\left(4+c_{1}\right)\left(2+d_{1}\right),\left(4+c_{1}\right)\left(3+d_{1}+d_{2}\right)\right\}\right\}$ & 43 \\
\hline $27 I 4 / m$ & $52 / m_{z}$ & $1\{\{1,0,0\},\{0,1,0\},\{0,0,1\}\}$ & \\
\hline
\end{tabular}


Table A.1 - continued from previous page

\begin{tabular}{|c|c|c|c|}
\hline ACC & $P_{N}$ & $B_{N}$ matrix row by row & $\mathrm{c \#}$ \\
\hline $27 I 4 / m$ & $52 / m_{z}$ & $2\{\{1,0,0\},\{1,2,0\},\{0,0,2\}\}$ & \\
\hline $27 I 4 / m$ & $52 / m_{z}$ & $3\{\{1,0,0\},\{0,1,0\},\{1,1,2\}\}$ & \\
\hline $27 I 4 / m$ & $6 \overline{4}$ & $1\{\{1,0,0\},\{0,1,0\},\{3,3,4\}\}$ & \\
\hline $27 I 4 / m$ & $6 \overline{4}$ & $2\{\{1,0,0\},\{0,1,0\},\{1,1,2\}\}$ & \\
\hline $27 I 4 / m$ & $6 \overline{4}$ & $3\{\{1,0,0\},\{0,1,0\},\{0,0,1\}\}$ & \\
\hline $27 I 4 / m$ & $6 \overline{4}$ & $4\{\{1,0,0\},\{0,1,0\},\{3,3,4\}\}$ & \\
\hline $27 I 4 / m$ & $6 \overline{4}$ & $5\{\{1,0,0\},\{0,1,0\},\{1,1,2\}\}$ & \\
\hline $27 I 4 / m$ & $6 \overline{4}$ & $6\{\{1,0,0\},\{0,1,0\},\{0,0,1\}\}$ & \\
\hline $27 I 4 / m$ & 74 & $1\{\{1,0,0\},\{0,1,0\},\{0,0,1\}\}$ & \\
\hline $27 I 4 / m$ & 74 & $2\left\{\{1,0,0\},\{0,1,0\},\left\{1,1,2+c_{1}\right\}\right\}$ & \\
\hline $27 I 4 / m$ & $84 / m$ & $1\{\{1,0,0\},\{0,1,0\},\{1,1,2\}\}$ & \\
\hline $27 I 4 / m$ & $84 / m$ & $2\{\{1,0,0\},\{0,1,0\},\{0,0,1\}\}$ & \\
\hline $28 P 422$ & $22 z$ & $1\left\{\{1,0,0\},\{0,1,0\},\left\{1+c_{1}, 1+c_{1}, 2+2 c_{1}\right\}\right\}$ & \\
\hline $28 P 422$ & $22 z$ & $2\left\{\{1,0,0\},\{0,1,0\},\left\{0,0,1+c_{1}\right\}\right\}$ & \\
\hline $28 P 422$ & $22 z$ & $3\left\{\{1,0,0\},\{1,2,0\},\left\{1+c_{1}, 0,2+2 c_{1}\right\}\right\}$ & \\
\hline $28 P 422$ & $22 z$ & $4\left\{\{1,0,0\},\{1,2,0\},\left\{0,0,1+c_{1}\right\}\right\}$ & \\
\hline $28 P 422$ & $22_{z}$ & $5\left\{\{2,0,0\},\{0,2,0\},\left\{0,0,1+c_{1}\right\}\right\}$ & \\
\hline $28 P 422$ & 3222 & $1\{\{1,0,0\},\{0,1,0\},\{0,0,1\}\}$ & \\
\hline $28 P 422$ & 3222 & $2\{\{1,0,0\},\{0,1,0\},\{1,1,2\}\}$ & \\
\hline $28 P 422$ & 3222 & $3\{\{1,0,0\},\{0,1,0\},\{0,0,2\}\}$ & \\
\hline $28 P 422$ & 3222 & $4\{\{1,0,0\},\{1,2,0\},\{0,0,1\}\}$ & \\
\hline $28 P 422$ & 3222 & $5\{\{1,0,0\},\{1,2,0\},\{1,0,2\}\}$ & \\
\hline $28 P 422$ & 3222 & $6\{\{1,0,0\},\{1,2,0\},\{0,0,2\}\}$ & \\
\hline $28 P 422$ & 3222 & $7\{\{2,0,0\},\{0,2,0\},\{0,0,1\}\}$ & \\
\hline $28 P 422$ & 3222 & $8\{\{2,0,0\},\{0,2,0\},\{0,0,2\}\}$ & \\
\hline $28 P 422$ & $4222_{d}$ & $1\{\{1,0,0\},\{1,2,0\},\{0,0,1\}\}$ & \\
\hline $28 P 422$ & $4222_{d}$ & $2\{\{1,0,0\},\{0,1,0\},\{0,0,1\}\}$ & \\
\hline $28 P 422$ & $4222_{d}$ & $3\{\{1,0,0\},\{1,2,0\},\{0,0,2\}\}$ & \\
\hline
\end{tabular}


Table A.1 - continued from previous page

\begin{tabular}{|c|c|c|c|}
\hline ACC & $P_{N}$ & $B_{N}$ matrix row by row & $\mathrm{c \#}$ \\
\hline $28 P 422$ & $4222_{d}$ & $4\{\{1,0,0\},\{0,1,0\},\{1,1,2\}\}$ & \\
\hline $28 P 422$ & $4222_{d}$ & $5\{\{1,0,0\},\{0,1,0\},\{0,0,2\}\}$ & \\
\hline $28 P 422$ & 54 & $1\left\{\{1,0,0\},\{1,2,0\},\left\{0,0,1+c_{1}\right\}\right\}$ & \\
\hline $28 P 422$ & 54 & $2\left\{\{1,0,0\},\{0,1,0\},\left\{1+c_{1}, 1+c_{1}, 2+2 c_{1}\right\}\right\}$ & \\
\hline $28 P 422$ & 54 & $3\left\{\{1,0,0\},\{0,1,0\},\left\{0,0,1+c_{1}\right\}\right\}$ & \\
\hline $28 P 422$ & 6422 & $1\{\{1,0,0\},\{1,2,0\},\{0,0,1\}\}$ & \\
\hline $28 P 422$ & 6422 & $2\{\{1,0,0\},\{0,1,0\},\{0,0,1\}\}$ & \\
\hline $28 P 422$ & 6422 & $3\{\{1,0,0\},\{1,2,0\},\{0,0,2\}\}$ & \\
\hline $28 P 422$ & 6422 & $4\{\{1,0,0\},\{0,1,0\},\{1,1,2\}\}$ & \\
\hline $28 P 422$ & 6422 & $5\{\{1,0,0\},\{0,1,0\},\{0,0,2\}\}$ & \\
\hline $29 I 422$ & $22 z$ & $1\{\{1,0,0\},\{1,2,0\},\{0,0,2\}\}$ & \\
\hline $29 I 422$ & $22_{z}$ & $2\{\{1,0,0\},\{0,1,0\},\{0,0,1\}\}$ & \\
\hline $29 I 422$ & $22 z$ & $3\left\{\{1,0,0\},\{1,2,0\},\left\{2,2,2\left(2+c_{1}\right)\right\}\right\}$ & \\
\hline $29 I 422$ & $22 z$ & $4\left\{\{1,0,0\},\{0,1,0\},\left\{3+2 c_{1}, 3+2 c_{1}, 4\left(1+c_{1}\right)\right\}\right\}$ & \\
\hline $29 I 422$ & $22 z$ & $5\left\{\{1,0,0\},\{0,1,0\},\left\{1,1,2+c_{1}\right\}\right\}$ & \\
\hline $29 I 422$ & 3222 & $1\{\{1,0,0\},\{0,1,0\},\{3,3,4\}\}$ & \\
\hline $29 I 422$ & 3222 & $2\{\{1,0,0\},\{0,1,0\},\{1,1,2\}\}$ & \\
\hline $29 I 422$ & 3222 & $3\{\{1,0,0\},\{0,1,0\},\{0,0,1\}\}$ & \\
\hline $29 I 422$ & 3222 & $4\{\{1,0,0\},\{0,1,0\},\{3,3,4\}\}$ & \\
\hline $29 I 422$ & 3222 & $5\{\{1,0,0\},\{0,1,0\},\{1,1,2\}\}$ & \\
\hline $29 I 422$ & 3222 & $6\{\{1,0,0\},\{0,1,0\},\{0,0,1\}\}$ & \\
\hline $29 I 422$ & $4222_{d}$ & $1\{\{1,0,0\},\{0,1,0\},\{0,0,1\}\}$ & \\
\hline $29 I 422$ & $4222_{d}$ & $2\{\{1,0,0\},\{1,2,0\},\{0,0,2\}\}$ & \\
\hline $29 I 422$ & $4222_{d}$ & $3\{\{1,0,0\},\{0,1,0\},\{1,1,2\}\}$ & \\
\hline $29 I 422$ & 54 & $1\{\{1,0,0\},\{0,1,0\},\{0,0,1\}\}$ & \\
\hline $29 I 422$ & 54 & $2\left\{\{1,0,0\},\{0,1,0\},\left\{1,1,2+c_{1}\right\}\right\}$ & \\
\hline $29 I 422$ & 6422 & $1\{\{1,0,0\},\{0,1,0\},\{1,1,2\}\}$ & \\
\hline $29 I 422$ & 6422 & $2\{\{1,0,0\},\{0,1,0\},\{0,0,1\}\}$ & \\
\hline
\end{tabular}


Table A.1 - continued from previous page

\begin{tabular}{|c|c|c|c|}
\hline ACC & $P_{N}$ & $B_{N}$ matrix row by row & $\mathrm{c \#}$ \\
\hline $30 P 4 \mathrm{~mm}$ & $22_{z}$ & $1\left\{\{1,0,0\},\{0,1,0\},\left\{1+c_{1}, 1+c_{1}, 2+2 c_{1}\right\}\right\}$ & \\
\hline $30 \mathrm{P} 4 \mathrm{~mm}$ & $22 z$ & $2\left\{\{1,0,0\},\{0,1,0\},\left\{0,0,1+c_{1}\right\}\right\}$ & \\
\hline $30 \mathrm{P} 4 \mathrm{~mm}$ & $22 z$ & $3\left\{\{1,0,0\},\{1,2,0\},\left\{1+c_{1}, 0,2+2 c_{1}\right\}\right\}$ & \\
\hline $30 P 4 m m$ & $22 z$ & $4\left\{\{1,0,0\},\{1,2,0\},\left\{0,0,1+c_{1}\right\}\right\}$ & \\
\hline $30 \mathrm{P} 4 \mathrm{~mm}$ & $22 z$ & $5\left\{\{2,0,0\},\{0,2,0\},\left\{0,0,1+c_{1}\right\}\right\}$ & \\
\hline $30 \mathrm{P} 4 \mathrm{~mm}$ & $3 m m 2_{z}$ & $1\left\{\{1,0,0\},\{0,1,0\},\left\{1+c_{1}, 1+c_{1}, 2+2 c_{1}\right\}\right\}$ & \\
\hline $30 \mathrm{P} 4 \mathrm{~mm}$ & $3 m m 2_{z}$ & $2\left\{\{1,0,0\},\{0,1,0\},\left\{0,0,1+c_{1}\right\}\right\}$ & \\
\hline $30 \mathrm{P} 4 \mathrm{~mm}$ & $3 m m 2_{z}$ & $3\left\{\{1,0,0\},\{1,2,0\},\left\{1+c_{1}, 0,2+2 c_{1}\right\}\right\}$ & \\
\hline $30 P 4 m m$ & $3 m m 2_{z}$ & $4\left\{\{1,0,0\},\{1,2,0\},\left\{0,0,1+c_{1}\right\}\right\}$ & \\
\hline $30 P 4 m m$ & $3 m m 2_{z}$ & $5\left\{\{2,0,0\},\{0,2,0\},\left\{0,0,1+c_{1}\right\}\right\}$ & \\
\hline $30 P 4 \mathrm{~mm}$ & $4 m m 2_{d}$ & $1\left\{\{1,0,0\},\{1,2,0\},\left\{0,0,1+c_{1}\right\}\right\}$ & \\
\hline $30 P 4 \mathrm{~mm}$ & $4 m m 2_{d}$ & $2\left\{\{1,0,0\},\{0,1,0\},\left\{1+c_{1}, 1+c_{1}, 2+2 c_{1}\right\}\right\}$ & \\
\hline $30 P 4 m m$ & $4 m m 2_{d}$ & $3\left\{\{1,0,0\},\{0,1,0\},\left\{0,0,1+c_{1}\right\}\right\}$ & \\
\hline $30 P 4 \mathrm{~mm}$ & 54 & $1\left\{\{1,0,0\},\{1,2,0\},\left\{0,0,1+c_{1}\right\}\right\}$ & \\
\hline $30 \mathrm{P} 4 \mathrm{~mm}$ & 54 & $2\left\{\{1,0,0\},\{0,1,0\},\left\{1+c_{1}, 1+c_{1}, 2+2 c_{1}\right\}\right\}$ & \\
\hline $30 \mathrm{P} 4 \mathrm{~mm}$ & 54 & $3\left\{\{1,0,0\},\{0,1,0\},\left\{0,0,1+c_{1}\right\}\right\}$ & \\
\hline $30 \mathrm{P} 4 \mathrm{~mm}$ & $64 m m$ & $1\left\{\{1,0,0\},\{1,2,0\},\left\{0,0,1+c_{1}\right\}\right\}$ & \\
\hline $30 \mathrm{P} 4 \mathrm{~mm}$ & $64 m m$ & $2\left\{\{1,0,0\},\{0,1,0\},\left\{1+c_{1}, 1+c_{1}, 2+2 c_{1}\right\}\right\}$ & \\
\hline $30 P 4 m m$ & $64 m m$ & $3\left\{\{1,0,0\},\{0,1,0\},\left\{0,0,1+c_{1}\right\}\right\}$ & \\
\hline $31 \mathrm{I} 4 \mathrm{~mm}$ & $22_{z}$ & $1\{\{1,0,0\},\{1,2,0\},\{0,0,2\}\}$ & \\
\hline $31 \mathrm{I} 4 \mathrm{~mm}$ & $22 z$ & $2\{\{1,0,0\},\{0,1,0\},\{0,0,1\}\}$ & \\
\hline $31 \mathrm{I} 4 \mathrm{~mm}$ & $22 z$ & $3\left\{\{1,0,0\},\{1,2,0\},\left\{2,2,2\left(2+c_{1}\right)\right\}\right\}$ & \\
\hline $31 \mathrm{I} 4 \mathrm{~mm}$ & $22 z$ & $4\left\{\{1,0,0\},\{0,1,0\},\left\{3+2 c_{1}, 3+2 c_{1}, 4\left(1+c_{1}\right)\right\}\right\}$ & \\
\hline $31 \mathrm{I} 4 \mathrm{~mm}$ & $22_{z}$ & $5\left\{\{1,0,0\},\{0,1,0\},\left\{1,1,2+c_{1}\right\}\right\}$ & \\
\hline $31 \mathrm{I} 4 \mathrm{~mm}$ & $3 m m 2_{z}$ & $1\{\{1,0,0\},\{0,1,0\},\{0,0,1\}\}$ & \\
\hline $31 \mathrm{I} 4 \mathrm{~mm}$ & $3 m m 2_{z}$ & $2\left\{\{1,0,0\},\{0,1,0\},\left\{1,1,2+c_{1}\right\}\right\}$ & \\
\hline $31 \mathrm{I} 4 \mathrm{~mm}$ & $4 m m 2_{d}$ & $1\{\{1,0,0\},\{1,2,0\},\{0,0,2\}\}$ & \\
\hline $31 \mathrm{I} 4 \mathrm{~mm}$ & $4 m m 2_{d}$ & $2\{\{1,0,0\},\{0,1,0\},\{0,0,1\}\}$ & \\
\hline
\end{tabular}


Table A.1 - continued from previous page

\begin{tabular}{|c|c|c|c|}
\hline ACC & $P_{N}$ & $B_{N}$ matrix row by row & $\mathrm{c \#}$ \\
\hline $31 \mathrm{I} 4 \mathrm{~mm}$ & $4 m m 2_{d}$ & $3\left\{\{1,0,0\},\{1,2,0\},\left\{2,2,2\left(2+c_{1}\right)\right\}\right\}$ & \\
\hline $31 \mathrm{I} 4 \mathrm{~mm}$ & $4 m m 2_{d}$ & $4\left\{\{1,0,0\},\{0,1,0\},\left\{3+2 c_{1}, 3+2 c_{1}, 4\left(1+c_{1}\right)\right\}\right\}$ & \\
\hline $31 \mathrm{I} 4 \mathrm{~mm}$ & $4 m m 2_{d}$ & $5\left\{\{1,0,0\},\{0,1,0\},\left\{1,1,2+c_{1}\right\}\right\}$ & \\
\hline $31 \mathrm{I} 4 \mathrm{~mm}$ & 54 & $1\{\{1,0,0\},\{0,1,0\},\{0,0,1\}\}$ & \\
\hline $31 \mathrm{I} 4 \mathrm{~mm}$ & 54 & $2\left\{\{1,0,0\},\{0,1,0\},\left\{1,1,2+c_{1}\right\}\right\}$ & \\
\hline $31 \mathrm{I} 4 \mathrm{~mm}$ & $64 m m$ & $1\{\{1,0,0\},\{0,1,0\},\{0,0,1\}\}$ & \\
\hline $31 \mathrm{I} 4 \mathrm{~mm}$ & $64 m m$ & $2\left\{\{1,0,0\},\{0,1,0\},\left\{1,1,2+c_{1}\right\}\right\}$ & \\
\hline $32 P \overline{4} 2 m$ & $22_{z}$ & $1\left\{\{1,0,0\},\{0,1,0\},\left\{1+c_{1}, 1+c_{1}, 2+2 c_{1}\right\}\right\}$ & \\
\hline $32 P \overline{4} 2 m$ & $22 z$ & $2\left\{\{1,0,0\},\{0,1,0\},\left\{0,0,1+c_{1}\right\}\right\}$ & \\
\hline $32 P \overline{4} 2 m$ & $22 z$ & $3\left\{\{1,0,0\},\{1,2,0\},\left\{1+c_{1}, 0,2+2 c_{1}\right\}\right\}$ & \\
\hline $32 P \overline{4} 2 m$ & $22 z$ & $4\left\{\{1,0,0\},\{1,2,0\},\left\{0,0,1+c_{1}\right\}\right\}$ & \\
\hline $32 P \overline{4} 2 m$ & $22 z$ & $5\left\{\{2,0,0\},\{0,2,0\},\left\{0,0,1+c_{1}\right\}\right\}$ & \\
\hline $32 P \overline{4} 2 m$ & 3222 & $1\{\{1,0,0\},\{0,1,0\},\{0,0,1\}\}$ & \\
\hline $32 P \overline{4} 2 m$ & 3222 & $2\{\{1,0,0\},\{0,1,0\},\{1,1,2\}\}$ & \\
\hline $32 P \overline{4} 2 m$ & 3222 & $3\{\{1,0,0\},\{0,1,0\},\{0,0,2\}\}$ & \\
\hline $32 P \overline{4} 2 m$ & 3222 & $4\{\{1,0,0\},\{1,2,0\},\{0,0,1\}\}$ & \\
\hline $32 P \overline{4} 2 m$ & 3222 & $5\{\{1,0,0\},\{1,2,0\},\{1,0,2\}\}$ & \\
\hline $32 P \overline{4} 2 m$ & 3222 & $6\{\{1,0,0\},\{1,2,0\},\{0,0,2\}\}$ & \\
\hline $32 P \overline{4} 2 m$ & 3222 & $7\{\{2,0,0\},\{0,2,0\},\{0,0,1\}\}$ & \\
\hline $32 P \overline{4} 2 m$ & 3222 & $8\{\{2,0,0\},\{0,2,0\},\{0,0,2\}\}$ & \\
\hline $32 P \overline{4} 2 m$ & $4 m m 2_{d}$ & $1\left\{\{1,0,0\},\{1,2,0\},\left\{0,0,1+c_{1}\right\}\right\}$ & \\
\hline $32 P \overline{4} 2 m$ & $4 m m 2_{d}$ & $2\left\{\{1,0,0\},\{0,1,0\},\left\{1+c_{1}, 1+c_{1}, 2+2 c_{1}\right\}\right\}$ & \\
\hline $32 P \overline{4} 2 m$ & $4 m m 2_{d}$ & $3\left\{\{1,0,0\},\{0,1,0\},\left\{0,0,1+c_{1}\right\}\right\}$ & \\
\hline $32 P \overline{4} 2 m$ & $5 \overline{4}$ & $1\{\{1,0,0\},\{1,2,0\},\{0,0,1\}\}$ & \\
\hline $32 P \overline{4} 2 m$ & $5 \overline{4}$ & $2\{\{1,0,0\},\{0,1,0\},\{0,0,1\}\}$ & \\
\hline $32 P \overline{4} 2 m$ & $5 \overline{4}$ & $3\{\{1,0,0\},\{1,2,0\},\{0,0,2\}\}$ & \\
\hline $32 P \overline{4} 2 m$ & $5 \overline{4}$ & $4\{\{1,0,0\},\{0,1,0\},\{1,1,2\}\}$ & \\
\hline $32 P \overline{4} 2 m$ & $5 \overline{4}$ & $5\{\{1,0,0\},\{0,1,0\},\{0,0,2\}\}$ & \\
\hline
\end{tabular}


Table A.1 - continued from previous page

\begin{tabular}{|c|c|c|c|}
\hline ACC & $P_{N}$ & $B_{N}$ matrix row by row & $\mathrm{c \#}$ \\
\hline $32 P \overline{4} 2 m$ & $6 \overline{4} 2 m$ & $1\{\{1,0,0\},\{1,2,0\},\{0,0,1\}\}$ & \\
\hline $32 P \overline{4} 2 m$ & $6 \overline{4} 2 m$ & $2\{\{1,0,0\},\{0,1,0\},\{0,0,1\}\}$ & \\
\hline $32 P \overline{4} 2 m$ & $6 \overline{4} 2 m$ & $3\{\{1,0,0\},\{1,2,0\},\{0,0,2\}\}$ & \\
\hline $32 P \overline{4} 2 m$ & $6 \overline{4} 2 m$ & $4\{\{1,0,0\},\{0,1,0\},\{1,1,2\}\}$ & \\
\hline $32 P \overline{4} 2 m$ & $6 \overline{4} 2 m$ & $5\{\{1,0,0\},\{0,1,0\},\{0,0,2\}\}$ & \\
\hline $33 P \overline{4} m 2$ & $22_{z}$ & $1\left\{\{1,0,0\},\{0,1,0\},\left\{1+c_{1}, 1+c_{1}, 2+2 c_{1}\right\}\right\}$ & \\
\hline $33 P \overline{4} m 2$ & $22_{z}$ & $2\left\{\{1,0,0\},\{0,1,0\},\left\{0,0,1+c_{1}\right\}\right\}$ & \\
\hline $33 P \overline{4} m 2$ & $22_{z}$ & $3\left\{\{1,0,0\},\{1,2,0\},\left\{1+c_{1}, 0,2+2 c_{1}\right\}\right\}$ & \\
\hline $33 P \overline{4} m 2$ & $22 z$ & $4\left\{\{1,0,0\},\{1,2,0\},\left\{0,0,1+c_{1}\right\}\right\}$ & \\
\hline $33 P \overline{4} m 2$ & $22_{z}$ & $5\left\{\{2,0,0\},\{0,2,0\},\left\{0,0,1+c_{1}\right\}\right\}$ & \\
\hline $33 P \overline{4} m 2$ & $3 m m 2_{z}$ & $1\left\{\{1,0,0\},\{0,1,0\},\left\{1+c_{1}, 1+c_{1}, 2+2 c_{1}\right\}\right\}$ & \\
\hline $33 P \overline{4} m 2$ & $3 m m 2_{z}$ & $2\left\{\{1,0,0\},\{0,1,0\},\left\{0,0,1+c_{1}\right\}\right\}$ & \\
\hline $33 P \overline{4} m 2$ & $3 m m 2_{z}$ & $3\left\{\{1,0,0\},\{1,2,0\},\left\{1+c_{1}, 0,2+2 c_{1}\right\}\right\}$ & \\
\hline $33 P \overline{4} m 2$ & $3 m m 2_{z}$ & $4\left\{\{1,0,0\},\{1,2,0\},\left\{0,0,1+c_{1}\right\}\right\}$ & \\
\hline $33 P \overline{4} m 2$ & $3 m m 2_{z}$ & $5\left\{\{2,0,0\},\{0,2,0\},\left\{0,0,1+c_{1}\right\}\right\}$ & \\
\hline $33 P \overline{4} m 2$ & $4222_{d}$ & $1\{\{1,0,0\},\{1,2,0\},\{0,0,1\}\}$ & \\
\hline $33 P \overline{4} m 2$ & $4222_{d}$ & $2\{\{1,0,0\},\{0,1,0\},\{0,0,1\}\}$ & \\
\hline $33 P \overline{4} m 2$ & $4222_{d}$ & $3\{\{1,0,0\},\{1,2,0\},\{0,0,2\}\}$ & \\
\hline $33 P \overline{4} m 2$ & $4222_{d}$ & $4\{\{1,0,0\},\{0,1,0\},\{1,1,2\}\}$ & \\
\hline $33 P \overline{4} m 2$ & $4222_{d}$ & $5\{\{1,0,0\},\{0,1,0\},\{0,0,2\}\}$ & \\
\hline $33 P \overline{4} m 2$ & $5 \overline{4}$ & $1\{\{1,0,0\},\{1,2,0\},\{0,0,1\}\}$ & \\
\hline $33 P \overline{4} m 2$ & $5 \overline{4}$ & $2\{\{1,0,0\},\{0,1,0\},\{0,0,1\}\}$ & \\
\hline $33 P \overline{4} m 2$ & $5 \overline{4}$ & $3\{\{1,0,0\},\{1,2,0\},\{0,0,2\}\}$ & \\
\hline $33 P \overline{4} m 2$ & $5 \overline{4}$ & $4\{\{1,0,0\},\{0,1,0\},\{1,1,2\}\}$ & \\
\hline $33 P \overline{4} m 2$ & $5 \overline{4}$ & $5\{\{1,0,0\},\{0,1,0\},\{0,0,2\}\}$ & \\
\hline $33 P \overline{4} m 2$ & $6 \overline{4} m 2$ & $1\{\{1,0,0\},\{1,2,0\},\{0,0,1\}\}$ & \\
\hline $33 P \overline{4} m 2$ & $6 \overline{4} m 2$ & $2\{\{1,0,0\},\{0,1,0\},\{0,0,1\}\}$ & \\
\hline $33 P \overline{4} m 2$ & $6 \overline{4} m 2$ & $3\{\{1,0,0\},\{1,2,0\},\{0,0,2\}\}$ & \\
\hline
\end{tabular}


Table A.1 - continued from previous page

\begin{tabular}{|c|c|c|c|}
\hline ACC & $P_{N}$ & $B_{N}$ matrix row by row & $\mathrm{c \#}$ \\
\hline $33 P \overline{4} m 2$ & $6 \overline{4} m 2$ & $4\{\{1,0,0\},\{0,1,0\},\{1,1,2\}\}$ & \\
\hline $33 P \overline{4} m 2$ & $6 \overline{4} m 2$ & $5\{\{1,0,0\},\{0,1,0\},\{0,0,2\}\}$ & \\
\hline $34 I \overline{4} m 2$ & $22 z$ & $1\{\{1,0,0\},\{1,2,0\},\{0,0,2\}\}$ & \\
\hline $34 I \overline{4} m 2$ & $22 z$ & $2\{\{1,0,0\},\{0,1,0\},\{0,0,1\}\}$ & \\
\hline $34 I \overline{4} m 2$ & $22 z$ & $3\left\{\{1,0,0\},\{1,2,0\},\left\{2,2,2\left(2+c_{1}\right)\right\}\right\}$ & \\
\hline $34 I \overline{4} m 2$ & $22_{z}$ & $4\left\{\{1,0,0\},\{0,1,0\},\left\{3+2 c_{1}, 3+2 c_{1}, 4\left(1+c_{1}\right)\right\}\right\}$ & \\
\hline $34 I \overline{4} m 2$ & $22_{z}$ & $5\left\{\{1,0,0\},\{0,1,0\},\left\{1,1,2+c_{1}\right\}\right\}$ & \\
\hline $34 I \overline{4} m 2$ & $3 m m 2_{z}$ & $1\{\{1,0,0\},\{0,1,0\},\{0,0,1\}\}$ & \\
\hline $34 I \overline{4} m 2$ & $3 m m 2_{z}$ & $2\left\{\{1,0,0\},\{0,1,0\},\left\{1,1,2+c_{1}\right\}\right\}$ & \\
\hline $34 I \overline{4} m 2$ & $4222_{d}$ & $1\{\{1,0,0\},\{0,1,0\},\{0,0,1\}\}$ & \\
\hline $34 I \overline{4} m 2$ & $4222_{d}$ & $2\{\{1,0,0\},\{1,2,0\},\{0,0,2\}\}$ & \\
\hline $34 I \overline{4} m 2$ & $4222_{d}$ & $3\{\{1,0,0\},\{0,1,0\},\{1,1,2\}\}$ & \\
\hline $34 I \overline{4} m 2$ & $5 \overline{4}$ & $1\{\{1,0,0\},\{0,1,0\},\{3,3,4\}\}$ & \\
\hline $34 I \overline{4} m 2$ & $5 \overline{4}$ & $2\{\{1,0,0\},\{0,1,0\},\{1,1,2\}\}$ & \\
\hline $34 I \overline{4} m 2$ & $5 \overline{4}$ & $3\{\{1,0,0\},\{0,1,0\},\{0,0,1\}\}$ & \\
\hline $34 I \overline{4} m 2$ & $5 \overline{4}$ & $4\{\{1,0,0\},\{0,1,0\},\{3,3,4\}\}$ & \\
\hline $34 I \overline{4} m 2$ & $5 \overline{4}$ & $5\{\{1,0,0\},\{0,1,0\},\{1,1,2\}\}$ & \\
\hline $34 I \overline{4} m 2$ & $5 \overline{4}$ & $6\{\{1,0,0\},\{0,1,0\},\{0,0,1\}\}$ & \\
\hline $34 I \overline{4} m 2$ & $6 \overline{4} m 2$ & $1\{\{1,0,0\},\{0,1,0\},\{1,1,2\}\}$ & \\
\hline $34 I \overline{4} m 2$ & $6 \overline{4} m 2$ & $2\{\{1,0,0\},\{0,1,0\},\{0,0,1\}\}$ & \\
\hline $35 I \overline{4} 2 m$ & $22 z$ & $1\{\{1,0,0\},\{1,2,0\},\{0,0,2\}\}$ & \\
\hline $35 I \overline{4} 2 m$ & $22 z$ & $2\{\{1,0,0\},\{0,1,0\},\{0,0,1\}\}$ & \\
\hline $35 I \overline{4} 2 m$ & $22 z$ & $3\left\{\{1,0,0\},\{1,2,0\},\left\{2,2,2\left(2+c_{1}\right)\right\}\right\}$ & \\
\hline $35 I \overline{4} 2 m$ & $22 z$ & $4\left\{\{1,0,0\},\{0,1,0\},\left\{3+2 c_{1}, 3+2 c_{1}, 4\left(1+c_{1}\right)\right\}\right\}$ & \\
\hline $35 I \overline{4} 2 m$ & $22 z$ & $5\left\{\{1,0,0\},\{0,1,0\},\left\{1,1,2+c_{1}\right\}\right\}$ & \\
\hline $35 I \overline{4} 2 m$ & 3222 & $1\{\{1,0,0\},\{0,1,0\},\{3,3,4\}\}$ & \\
\hline $35 I \overline{4} 2 m$ & 3222 & $2\{\{1,0,0\},\{0,1,0\},\{1,1,2\}\}$ & \\
\hline $35 I \overline{4} 2 m$ & 3222 & $3\{\{1,0,0\},\{0,1,0\},\{0,0,1\}\}$ & \\
\hline
\end{tabular}


Table A.1 - continued from previous page

\begin{tabular}{|c|c|c|c|}
\hline ACC & $P_{N}$ & $B_{N}$ matrix row by row & $\mathrm{c \#}$ \\
\hline $35 I \overline{4} 2 m$ & 3222 & $4\{\{1,0,0\},\{0,1,0\},\{3,3,4\}\}$ & \\
\hline $35 I \overline{4} 2 m$ & 3222 & $5\{\{1,0,0\},\{0,1,0\},\{1,1,2\}\}$ & \\
\hline $35 I \overline{4} 2 m$ & 3222 & $6\{\{1,0,0\},\{0,1,0\},\{0,0,1\}\}$ & \\
\hline $35 I \overline{4} 2 m$ & $4 m m 2_{d}$ & $1\{\{1,0,0\},\{1,2,0\},\{0,0,2\}\}$ & \\
\hline $35 I \overline{4} 2 m$ & $4 m m 2_{d}$ & $2\{\{1,0,0\},\{0,1,0\},\{0,0,1\}\}$ & \\
\hline $35 I \overline{4} 2 m$ & $4 m m 2_{d}$ & $3\left\{\{1,0,0\},\{1,2,0\},\left\{2,2,2\left(2+c_{1}\right)\right\}\right\}$ & \\
\hline $35 I \overline{4} 2 m$ & $4 m m 2_{d}$ & $4\left\{\{1,0,0\},\{0,1,0\},\left\{3+2 c_{1}, 3+2 c_{1}, 4\left(1+c_{1}\right)\right\}\right\}$ & \\
\hline $35 I \overline{4} 2 m$ & $4 m m 2_{d}$ & $5\left\{\{1,0,0\},\{0,1,0\},\left\{1,1,2+c_{1}\right\}\right\}$ & \\
\hline $35 I \overline{4} 2 m$ & $5 \overline{4}$ & $1\{\{1,0,0\},\{0,1,0\},\{3,3,4\}\}$ & \\
\hline $35 I \overline{4} 2 m$ & $5 \overline{4}$ & $2\{\{1,0,0\},\{0,1,0\},\{1,1,2\}\}$ & \\
\hline $35 I \overline{4} 2 m$ & $5 \overline{4}$ & $3\{\{1,0,0\},\{0,1,0\},\{0,0,1\}\}$ & \\
\hline $35 I \overline{4} 2 m$ & $5 \overline{4}$ & $4\{\{1,0,0\},\{0,1,0\},\{3,3,4\}\}$ & \\
\hline $35 I \overline{4} 2 m$ & $5 \overline{4}$ & $5\{\{1,0,0\},\{0,1,0\},\{1,1,2\}\}$ & \\
\hline $35 I \overline{4} 2 m$ & $5 \overline{4}$ & $6\{\{1,0,0\},\{0,1,0\},\{0,0,1\}\}$ & \\
\hline $35 I \overline{4} 2 m$ & $6 \overline{4} 2 m$ & $1\{\{1,0,0\},\{0,1,0\},\{3,3,4\}\}$ & \\
\hline $35 I \overline{4} 2 m$ & $6 \overline{4} 2 m$ & $2\{\{1,0,0\},\{0,1,0\},\{1,1,2\}\}$ & \\
\hline $35 I \overline{4} 2 m$ & $6 \overline{4} 2 m$ & $3\{\{1,0,0\},\{0,1,0\},\{0,0,1\}\}$ & \\
\hline $35 I \overline{4} 2 m$ & $6 \overline{4} 2 m$ & $4\{\{1,0,0\},\{0,1,0\},\{3,3,4\}\}$ & \\
\hline $35 I \overline{4} 2 m$ & $6 \overline{4} 2 m$ & $5\{\{1,0,0\},\{0,1,0\},\{1,1,2\}\}$ & \\
\hline $35 I \overline{4} 2 m$ & $6 \overline{4} 2 m$ & $6\{\{1,0,0\},\{0,1,0\},\{0,0,1\}\}$ & \\
\hline $36 \mathrm{P} 4 / \mathrm{mmm}$ & $2 \overline{1}$ & $1\{\{1,0,0\},\{0,1,0\},\{0,0,1\}\}$ & \\
\hline $36 \mathrm{P} 4 / \mathrm{mmm}$ & $2 \overline{1}$ & $2\{\{1,0,0\},\{0,1,0\},\{1,1,2\}\}$ & \\
\hline $36 \mathrm{P} 4 / \mathrm{mmm}$ & $2 \overline{1}$ & $3\{\{1,0,0\},\{0,1,0\},\{0,0,2\}\}$ & \\
\hline $36 \mathrm{P} 4 / \mathrm{mmm}$ & $2 \overline{1}$ & $4\{\{1,0,0\},\{1,2,0\},\{0,0,1\}\}$ & \\
\hline $36 \mathrm{P} 4 / \mathrm{mmm}$ & $2 \overline{1}$ & $5\{\{1,0,0\},\{1,2,0\},\{1,0,2\}\}$ & \\
\hline $36 \mathrm{P} 4 / \mathrm{mmm}$ & $2 \overline{1}$ & $6\{\{1,0,0\},\{1,2,0\},\{0,0,2\}\}$ & \\
\hline $36 \mathrm{P} 4 / \mathrm{mmm}$ & $2 \overline{1}$ & $7\{\{2,0,0\},\{0,2,0\},\{0,0,1\}\}$ & \\
\hline $36 \mathrm{P} 4 / \mathrm{mmm}$ & $2 \overline{1}$ & $8\{\{2,0,0\},\{0,2,0\},\{0,0,2\}\}$ & \\
\hline
\end{tabular}


Table A.1 - continued from previous page

\begin{tabular}{|c|c|c|c|}
\hline ACC & $P_{N}$ & $B_{N}$ matrix row by row & $\mathrm{c} \#$ \\
\hline $36 \mathrm{P} 4 / \mathrm{mmm}$ & $32 z$ & $1\left\{\{1,0,0\},\{0,1,0\},\left\{1+c_{1}, 1+c_{1}, 2+2 c_{1}\right\}\right\}$ & \\
\hline $36 \mathrm{P} 4 / \mathrm{mmm}$ & $32 z$ & $2\left\{\{1,0,0\},\{0,1,0\},\left\{0,0,1+c_{1}\right\}\right\}$ & \\
\hline $36 \mathrm{P} 4 / \mathrm{mmm}$ & $32 z$ & $3\left\{\{1,0,0\},\{1,2,0\},\left\{1+c_{1}, 0,2+2 c_{1}\right\}\right\}$ & \\
\hline $36 \mathrm{P} 4 / \mathrm{mmm}$ & $32 z$ & $4\left\{\{1,0,0\},\{1,2,0\},\left\{0,0,1+c_{1}\right\}\right\}$ & \\
\hline $36 \mathrm{P} 4 / \mathrm{mmm}$ & $32 z$ & $5\left\{\{2,0,0\},\{0,2,0\},\left\{0,0,1+c_{1}\right\}\right\}$ & \\
\hline $36 \mathrm{P} 4 / \mathrm{mmm}$ & $4 m_{z}$ & $1\left\{\left\{2\left(1+c_{1}\right), 0,0\right\},\left\{0,2\left(1+c_{1}\right), 0\right\},\{0,0,1\}\right\}$ & \\
\hline $36 \mathrm{P} 4 / \mathrm{mmm}$ & $4 m_{z}$ & $2\left\{\left\{2\left(1+c_{1}\right), 0,0\right\},\left\{2\left(1+c_{1}\right), 4\left(1+c_{1}\right), 0\right\},\{0,0,1\}\right\}$ & \\
\hline $36 \mathrm{P} 4 / \mathrm{mmm}$ & $4 m_{z}$ & $3\left\{\left\{1+2 c_{1}, 0,0\right\},\left\{0,1+2 c_{1}, 0\right\},\{0,0,1\}\right\}$ & \\
\hline $36 \mathrm{P} 4 / \mathrm{mmm}$ & $4 m_{z}$ & $4\left\{\left\{1+2 c_{1}, 0,0\right\},\left\{1+2 c_{1}, 2+4 c_{1}, 0\right\},\{0,0,1\}\right\}$ & \\
\hline $36 \mathrm{P} 4 / \mathrm{mmm}$ & $4 m_{z}$ & $5\left\{\left\{2\left(1+c_{1}\right), 0,0\right\},\left\{2\left(1+c_{1}\right)\left(2+d_{1}\right), 4\left(1+c_{1}\right)\left(2+d_{1}\right), 0\right\},\{0,0,1\}\right\}$ & $2^{*}$ \\
\hline $36 \mathrm{P} 4 / \mathrm{mmm}$ & $4 m_{z}$ & $6\left\{\left\{1+2 c_{1}, 0,0\right\},\left\{\left(1+2 c_{1}\right)\left(2+d_{1}\right), 2\left(1+2 c_{1}\right)\left(2+d_{1}\right), 0\right\},\{0,0,1\}\right\}$ & $2^{*}$ \\
\hline $36 \mathrm{P} 4 / \mathrm{mmm}$ & $4 m_{z}$ & $7\left\{\left\{2\left(1+c_{1}\right), 0,0\right\},\left\{0,2\left(1+c_{1}\right), 0\right\},\{0,0,2\}\right\}$ & \\
\hline $36 \mathrm{P} 4 / \mathrm{mmm}$ & $4 m_{z}$ & $8\left\{\left\{2\left(1+c_{1}\right), 0,0\right\},\left\{2\left(1+c_{1}\right), 4\left(1+c_{1}\right), 0\right\},\{0,0,2\}\right\}$ & \\
\hline $36 \mathrm{P} 4 / \mathrm{mmm}$ & $4 m_{z}$ & $9\left\{\left\{1+2 c_{1}, 0,0\right\},\left\{0,1+2 c_{1}, 0\right\},\{0,0,2\}\right\}$ & \\
\hline $36 \mathrm{P} 4 / \mathrm{mmm}$ & $4 m_{z}$ & $10\left\{\left\{1+2 c_{1}, 0,0\right\},\left\{1+2 c_{1}, 2+4 c_{1}, 0\right\},\{0,0,2\}\right\}$ & \\
\hline $36 \mathrm{P} 4 / \mathrm{mmm}$ & $4 m_{z}$ & $11\left\{\left\{2\left(1+c_{1}\right), 0,0\right\},\left\{2\left(1+c_{1}\right)\left(2+d_{1}\right), 4\left(1+c_{1}\right)\left(2+d_{1}\right), 0\right\},\{0,0,2\}\right\}$ & $2^{*}$ \\
\hline $36 \mathrm{P} 4 / \mathrm{mmm}$ & $4 m_{z}$ & $12\left\{\left\{1+2 c_{1}, 0,0\right\},\left\{\left(1+2 c_{1}\right)\left(2+d_{1}\right), 2\left(1+2 c_{1}\right)\left(2+d_{1}\right), 0\right\},\{0,0,2\}\right\}$ & $2^{*}$ \\
\hline $36 \mathrm{P} 4 / \mathrm{mmm}$ & $4 m_{z}$ & $13\{\{1,0,0\},\{1,2,0\},\{1,0,2\}\}$ & \\
\hline $36 \mathrm{P} 4 / \mathrm{mmm}$ & $4 m_{z}$ & $14\left\{\left\{2+c_{1}, 0,0\right\},\left\{2+c_{1}, 2\left(2+c_{1}\right), 0\right\},\{1,0,2\}\right\}$ & \\
\hline $36 \mathrm{P} 4 / \mathrm{mmm}$ & $4 m_{z}$ & $15\left\{\{1,0,0\},\left\{2+d_{1}, 2\left(2+d_{1}\right), 0\right\},\{1,0,2\}\right\}$ & $1^{*}$ \\
\hline $36 \mathrm{P} 4 / \mathrm{mmm}$ & $4 m_{z}$ & $16\left\{\left\{1+c_{1}, 0,0\right\},\left\{0,1+c_{1}, 0\right\},\{1,1,2\}\right\}$ & \\
\hline $36 \mathrm{P} 4 / \mathrm{mmm}$ & $52 / m_{z}$ & $1\{\{1,0,0\},\{0,1,0\},\{0,0,1\}\}$ & \\
\hline $36 \mathrm{P} 4 / \mathrm{mmm}$ & $52 / m_{z}$ & $2\{\{1,0,0\},\{0,1,0\},\{1,1,2\}\}$ & \\
\hline $36 \mathrm{P} 4 / \mathrm{mmm}$ & $52 / m_{z}$ & $3\{\{1,0,0\},\{0,1,0\},\{0,0,2\}\}$ & \\
\hline $36 \mathrm{P} 4 / \mathrm{mmm}$ & $52 / m_{z}$ & $4\{\{1,0,0\},\{1,2,0\},\{0,0,1\}\}$ & \\
\hline $36 \mathrm{P} 4 / \mathrm{mmm}$ & $52 / m_{z}$ & $5\{\{1,0,0\},\{1,2,0\},\{1,0,2\}\}$ & \\
\hline $36 \mathrm{P} 4 / \mathrm{mmm}$ & $52 / m_{z}$ & $6\{\{1,0,0\},\{1,2,0\},\{0,0,2\}\}$ & \\
\hline $36 \mathrm{P} 4 / \mathrm{mmm}$ & $52 / m_{z}$ & $7\{\{2,0,0\},\{0,2,0\},\{0,0,1\}\}$ & \\
\hline
\end{tabular}


Table A.1 - continued from previous page

\begin{tabular}{|c|c|c|c|}
\hline ACC & $P_{N}$ & $B_{N}$ matrix row by row & $\mathrm{c \#}$ \\
\hline $36 \mathrm{P} 4 / \mathrm{mmm}$ & $52 / m_{z}$ & $8\{\{2,0,0\},\{0,2,0\},\{0,0,2\}\}$ & \\
\hline $36 \mathrm{P} 4 / \mathrm{mmm}$ & 6222 & $1\{\{1,0,0\},\{0,1,0\},\{0,0,1\}\}$ & \\
\hline $36 \mathrm{P} 4 / \mathrm{mmm}$ & 6222 & $2\{\{1,0,0\},\{0,1,0\},\{1,1,2\}\}$ & \\
\hline $36 \mathrm{P} 4 / \mathrm{mmm}$ & 6222 & $3\{\{1,0,0\},\{0,1,0\},\{0,0,2\}\}$ & \\
\hline $36 \mathrm{P} 4 / \mathrm{mmm}$ & 6222 & $4\{\{1,0,0\},\{1,2,0\},\{0,0,1\}\}$ & \\
\hline $36 \mathrm{P} 4 / \mathrm{mmm}$ & 6222 & $5\{\{1,0,0\},\{1,2,0\},\{1,0,2\}\}$ & \\
\hline $36 \mathrm{P} 4 / \mathrm{mmm}$ & 6222 & $6\{\{1,0,0\},\{1,2,0\},\{0,0,2\}\}$ & \\
\hline $36 \mathrm{P} 4 / \mathrm{mmm}$ & 6222 & $7\{\{2,0,0\},\{0,2,0\},\{0,0,1\}\}$ & \\
\hline $36 \mathrm{P} 4 / \mathrm{mmm}$ & 6222 & $8\{\{2,0,0\},\{0,2,0\},\{0,0,2\}\}$ & \\
\hline $36 \mathrm{P} 4 / \mathrm{mmm}$ & $7 \mathrm{~mm} 2_{z}$ & $1\left\{\{1,0,0\},\{0,1,0\},\left\{1+c_{1}, 1+c_{1}, 2+2 c_{1}\right\}\right\}$ & \\
\hline $36 \mathrm{P} 4 / \mathrm{mmm}$ & $7 m m 2_{z}$ & $2\left\{\{1,0,0\},\{0,1,0\},\left\{0,0,1+c_{1}\right\}\right\}$ & \\
\hline $36 \mathrm{P} 4 / \mathrm{mmm}$ & $7 m m 2_{z}$ & $3\left\{\{1,0,0\},\{1,2,0\},\left\{1+c_{1}, 0,2+2 c_{1}\right\}\right\}$ & \\
\hline $36 \mathrm{P} 4 / \mathrm{mmm}$ & $7 m m 2_{z}$ & $4\left\{\{1,0,0\},\{1,2,0\},\left\{0,0,1+c_{1}\right\}\right\}$ & \\
\hline $36 \mathrm{P} 4 / \mathrm{mmm}$ & $7 \mathrm{~mm} 2_{z}$ & $5\left\{\{2,0,0\},\{0,2,0\},\left\{0,0,1+c_{1}\right\}\right\}$ & \\
\hline $36 \mathrm{P} 4 / \mathrm{mmm}$ & $8222_{d}$ & $1\{\{1,0,0\},\{1,2,0\},\{0,0,1\}\}$ & \\
\hline $36 \mathrm{P} 4 / \mathrm{mmm}$ & $8222_{d}$ & $2\{\{1,0,0\},\{0,1,0\},\{0,0,1\}\}$ & \\
\hline $36 \mathrm{P} 4 / \mathrm{mmm}$ & $8222_{d}$ & $3\{\{1,0,0\},\{1,2,0\},\{0,0,2\}\}$ & \\
\hline $36 \mathrm{P} 4 / \mathrm{mmm}$ & $8222_{d}$ & $4\{\{1,0,0\},\{0,1,0\},\{1,1,2\}\}$ & \\
\hline $36 \mathrm{P} 4 / \mathrm{mmm}$ & $8222_{d}$ & $5\{\{1,0,0\},\{0,1,0\},\{0,0,2\}\}$ & \\
\hline $36 \mathrm{P} 4 / \mathrm{mmm}$ & $9 m m 2_{d}$ & $1\left\{\{1,0,0\},\{1,2,0\},\left\{0,0,1+c_{1}\right\}\right\}$ & \\
\hline $36 \mathrm{P} 4 / \mathrm{mmm}$ & $9 m m 2_{d}$ & $2\left\{\{1,0,0\},\{0,1,0\},\left\{1+c_{1}, 1+c_{1}, 2+2 c_{1}\right\}\right\}$ & \\
\hline $36 \mathrm{P} 4 / \mathrm{mmm}$ & $9 m m 2_{d}$ & $3\left\{\{1,0,0\},\{0,1,0\},\left\{0,0,1+c_{1}\right\}\right\}$ & \\
\hline $36 \mathrm{P} 4 / \mathrm{mmm}$ & $10 \overline{4}$ & $1\{\{1,0,0\},\{1,2,0\},\{0,0,1\}\}$ & \\
\hline $36 \mathrm{P} 4 / \mathrm{mmm}$ & $10 \overline{4}$ & $2\{\{1,0,0\},\{0,1,0\},\{0,0,1\}\}$ & \\
\hline $36 \mathrm{P} 4 / \mathrm{mmm}$ & $10 \overline{4}$ & $3\{\{1,0,0\},\{1,2,0\},\{0,0,2\}\}$ & \\
\hline $36 \mathrm{P} 4 / \mathrm{mmm}$ & $10 \overline{4}$ & $4\{\{1,0,0\},\{0,1,0\},\{1,1,2\}\}$ & \\
\hline $36 \mathrm{P} 4 / \mathrm{mmm}$ & $10 \overline{4}$ & $5\{\{1,0,0\},\{0,1,0\},\{0,0,2\}\}$ & \\
\hline $36 \mathrm{P} 4 / \mathrm{mmm}$ & 114 & $1\left\{\{1,0,0\},\{1,2,0\},\left\{0,0,1+c_{1}\right\}\right\}$ & \\
\hline
\end{tabular}


Table A.1 - continued from previous page

\begin{tabular}{|c|c|c|c|}
\hline ACC & $P_{N}$ & $B_{N}$ matrix row by row & $\mathrm{c} \#$ \\
\hline $36 \mathrm{P} 4 / \mathrm{mmm}$ & 114 & $2\left\{\{1,0,0\},\{0,1,0\},\left\{1+c_{1}, 1+c_{1}, 2+2 c_{1}\right\}\right\}$ & \\
\hline $36 \mathrm{P} 4 / \mathrm{mmm}$ & 114 & $3\left\{\{1,0,0\},\{0,1,0\},\left\{0,0,1+c_{1}\right\}\right\}$ & \\
\hline $36 \mathrm{P} 4 / \mathrm{mmm}$ & $12 \mathrm{mmm}$ & $1\{\{1,0,0\},\{0,1,0\},\{0,0,1\}\}$ & \\
\hline $36 \mathrm{P} 4 / \mathrm{mmm}$ & $12 \mathrm{mmm}$ & $2\{\{1,0,0\},\{0,1,0\},\{1,1,2\}\}$ & \\
\hline $36 \mathrm{P} 4 / \mathrm{mmm}$ & $12 \mathrm{mmm}$ & $3\{\{1,0,0\},\{0,1,0\},\{0,0,2\}\}$ & \\
\hline $36 \mathrm{P} 4 / \mathrm{mmm}$ & $12 \mathrm{mmm}$ & $4\{\{1,0,0\},\{1,2,0\},\{0,0,1\}\}$ & \\
\hline $36 \mathrm{P} 4 / \mathrm{mmm}$ & $12 \mathrm{mmm}$ & $5\{\{1,0,0\},\{1,2,0\},\{1,0,2\}\}$ & \\
\hline $36 \mathrm{P} 4 / \mathrm{mmm}$ & $12 \mathrm{mmm}$ & $6\{\{1,0,0\},\{1,2,0\},\{0,0,2\}\}$ & \\
\hline $36 \mathrm{P} 4 / \mathrm{mmm}$ & $12 \mathrm{mmm}$ & $7\{\{2,0,0\},\{0,2,0\},\{0,0,1\}\}$ & \\
\hline $36 \mathrm{P} 4 / \mathrm{mmm}$ & $12 \mathrm{mmm}$ & $8\{\{2,0,0\},\{0,2,0\},\{0,0,2\}\}$ & \\
\hline $36 \mathrm{P} 4 / \mathrm{mmm}$ & $13 \mathrm{mmm}_{d}$ & $1\{\{1,0,0\},\{1,2,0\},\{0,0,1\}\}$ & \\
\hline $36 \mathrm{P} 4 / \mathrm{mmm}$ & $13 \mathrm{mmm}_{d}$ & $2\{\{1,0,0\},\{0,1,0\},\{0,0,1\}\}$ & \\
\hline $36 \mathrm{P} 4 / \mathrm{mmm}$ & $13 \mathrm{mmm}_{d}$ & $3\{\{1,0,0\},\{1,2,0\},\{0,0,2\}\}$ & \\
\hline $36 \mathrm{P} 4 / \mathrm{mmm}$ & $13 \mathrm{mmm}_{d}$ & $4\{\{1,0,0\},\{0,1,0\},\{1,1,2\}\}$ & \\
\hline $36 \mathrm{P} 4 / \mathrm{mmm}$ & $13 \mathrm{mmm}_{d}$ & $5\{\{1,0,0\},\{0,1,0\},\{0,0,2\}\}$ & \\
\hline $36 \mathrm{P} 4 / \mathrm{mmm}$ & $144 / m$ & $1\{\{1,0,0\},\{1,2,0\},\{0,0,1\}\}$ & \\
\hline $36 \mathrm{P} 4 / \mathrm{mmm}$ & $144 / m$ & $2\{\{1,0,0\},\{0,1,0\},\{0,0,1\}\}$ & \\
\hline $36 \mathrm{P} 4 / \mathrm{mmm}$ & $144 / m$ & $3\{\{1,0,0\},\{1,2,0\},\{0,0,2\}\}$ & \\
\hline $36 \mathrm{P} 4 / \mathrm{mmm}$ & $144 / m$ & $4\{\{1,0,0\},\{0,1,0\},\{1,1,2\}\}$ & \\
\hline $36 \mathrm{P} 4 / \mathrm{mmm}$ & $144 / m$ & $5\{\{1,0,0\},\{0,1,0\},\{0,0,2\}\}$ & \\
\hline $36 \mathrm{P} 4 / \mathrm{mmm}$ & 15422 & $1\{\{1,0,0\},\{1,2,0\},\{0,0,1\}\}$ & \\
\hline $36 \mathrm{P} 4 / \mathrm{mmm}$ & 15422 & $2\{\{1,0,0\},\{0,1,0\},\{0,0,1\}\}$ & \\
\hline $36 \mathrm{P} 4 / \mathrm{mmm}$ & 15422 & $3\{\{1,0,0\},\{1,2,0\},\{0,0,2\}\}$ & \\
\hline $36 \mathrm{P} 4 / \mathrm{mmm}$ & 15422 & $4\{\{1,0,0\},\{0,1,0\},\{1,1,2\}\}$ & \\
\hline $36 \mathrm{P} 4 / \mathrm{mmm}$ & 15422 & $5\{\{1,0,0\},\{0,1,0\},\{0,0,2\}\}$ & \\
\hline $36 \mathrm{P} 4 / \mathrm{mmm}$ & $16 \overline{4} 2 m$ & $1\{\{1,0,0\},\{1,2,0\},\{0,0,1\}\}$ & \\
\hline $36 \mathrm{P} 4 / \mathrm{mmm}$ & $16 \overline{4} 2 m$ & $2\{\{1,0,0\},\{0,1,0\},\{0,0,1\}\}$ & \\
\hline $36 \mathrm{P} 4 / \mathrm{mmm}$ & $16 \overline{4} 2 m$ & $3\{\{1,0,0\},\{1,2,0\},\{0,0,2\}\}$ & \\
\hline
\end{tabular}


Table A.1 - continued from previous page

\begin{tabular}{|c|c|c|c|}
\hline ACC & $P_{N}$ & $B_{N}$ matrix row by row & $\mathrm{c \#}$ \\
\hline $36 \mathrm{P} 4 / \mathrm{mmm}$ & $16 \overline{4} 2 m$ & $4\{\{1,0,0\},\{0,1,0\},\{1,1,2\}\}$ & \\
\hline $36 \mathrm{P} 4 / \mathrm{mmm}$ & $16 \overline{4} 2 m$ & $5\{\{1,0,0\},\{0,1,0\},\{0,0,2\}\}$ & \\
\hline $36 \mathrm{P} 4 / \mathrm{mmm}$ & $17 \overline{4} m 2$ & $1\{\{1,0,0\},\{1,2,0\},\{0,0,1\}\}$ & \\
\hline $36 \mathrm{P} 4 / \mathrm{mmm}$ & $17 \overline{4} m 2$ & $2\{\{1,0,0\},\{0,1,0\},\{0,0,1\}\}$ & \\
\hline $36 \mathrm{P} 4 / \mathrm{mmm}$ & $17 \overline{4} m 2$ & $3\{\{1,0,0\},\{1,2,0\},\{0,0,2\}\}$ & \\
\hline $36 \mathrm{P} 4 / \mathrm{mmm}$ & $17 \overline{4} m 2$ & $4\{\{1,0,0\},\{0,1,0\},\{1,1,2\}\}$ & \\
\hline $36 \mathrm{P} 4 / \mathrm{mmm}$ & $17 \overline{4} m 2$ & $5\{\{1,0,0\},\{0,1,0\},\{0,0,2\}\}$ & \\
\hline $36 \mathrm{P} 4 / \mathrm{mmm}$ & $184 m m$ & $1\left\{\{1,0,0\},\{1,2,0\},\left\{0,0,1+c_{1}\right\}\right\}$ & \\
\hline $36 \mathrm{P} 4 / \mathrm{mmm}$ & $184 \mathrm{~mm}$ & $2\left\{\{1,0,0\},\{0,1,0\},\left\{1+c_{1}, 1+c_{1}, 2+2 c_{1}\right\}\right\}$ & \\
\hline $36 \mathrm{P} 4 / \mathrm{mmm}$ & $184 \mathrm{~mm}$ & $3\left\{\{1,0,0\},\{0,1,0\},\left\{0,0,1+c_{1}\right\}\right\}$ & \\
\hline $36 \mathrm{P} 4 / \mathrm{mmm}$ & $194 / \mathrm{mmm}$ & $1\{\{1,0,0\},\{1,2,0\},\{0,0,1\}\}$ & \\
\hline $36 \mathrm{P} 4 / \mathrm{mmm}$ & $194 / \mathrm{mmm}$ & $2\{\{1,0,0\},\{0,1,0\},\{0,0,1\}\}$ & \\
\hline $36 \mathrm{P} 4 / \mathrm{mmm}$ & $194 / \mathrm{mmm}$ & $3\{\{1,0,0\},\{1,2,0\},\{0,0,2\}\}$ & \\
\hline $36 \mathrm{P} 4 / \mathrm{mmm}$ & $194 / \mathrm{mmm}$ & $4\{\{1,0,0\},\{0,1,0\},\{1,1,2\}\}$ & \\
\hline $36 \mathrm{P} 4 / \mathrm{mmm}$ & $194 / \mathrm{mmm}$ & $5\{\{1,0,0\},\{0,1,0\},\{0,0,2\}\}$ & \\
\hline $37 \mathrm{I} / \mathrm{mmm}$ & $2 \overline{1}$ & $1\{\{1,0,0\},\{0,1,0\},\{0,0,1\}\}$ & \\
\hline $37 \mathrm{I} 4 / \mathrm{mmm}$ & $2 \overline{1}$ & $2\{\{1,0,0\},\{0,1,0\},\{1,1,2\}\}$ & \\
\hline $37 \mathrm{I} 4 / \mathrm{mmm}$ & $2 \overline{1}$ & $3\{\{1,0,0\},\{1,2,0\},\{0,0,2\}\}$ & \\
\hline $37 \mathrm{I} / \mathrm{mmm}$ & $2 \overline{1}$ & $4\{\{2,0,0\},\{0,2,0\},\{0,0,2\}\}$ & \\
\hline $37 \mathrm{I} / \mathrm{mmm}$ & $32 z$ & $1\{\{1,0,0\},\{1,2,0\},\{0,0,2\}\}$ & \\
\hline $37 \mathrm{I} / \mathrm{mmm}$ & $32 z$ & $2\{\{1,0,0\},\{0,1,0\},\{0,0,1\}\}$ & \\
\hline $37 \mathrm{I} 4 / \mathrm{mmm}$ & $32 z$ & $3\left\{\{1,0,0\},\{1,2,0\},\left\{2,2,2\left(2+c_{1}\right)\right\}\right\}$ & \\
\hline $37 \mathrm{I} / \mathrm{mmm}$ & $32 z$ & $4\left\{\{1,0,0\},\{0,1,0\},\left\{3+2 c_{1}, 3+2 c_{1}, 4\left(1+c_{1}\right)\right\}\right\}$ & \\
\hline $37 \mathrm{I} / \mathrm{mmm}$ & $32 z$ & $5\left\{\{1,0,0\},\{0,1,0\},\left\{1,1,2+c_{1}\right\}\right\}$ & \\
\hline $37 \mathrm{I} / \mathrm{mmm}$ & $4 m_{z}$ & $1\{\{1,0,0\},\{0,1,0\},\{0,0,1\}\}$ & \\
\hline $37 \mathrm{I} / \mathrm{mmm}$ & $4 m_{z}$ & $2\left\{\{1,0,0\},\{0,1,0\},\left\{1+d_{1}, 1+d_{2}, 2+d_{1}+d_{2}\right\}\right\}$ & 22 \\
\hline $37 \mathrm{I} / \mathrm{mmm}$ & $4 m_{z}$ & $3\{\{1,0,0\},\{1,2,0\},\{0,0,2\}\}$ & \\
\hline $37 \mathrm{I} 4 / \mathrm{mmm}$ & $4 m_{z}$ & $4\{\{1,0,0\},\{1,2,0\},\{0,2,4\}\}$ & \\
\hline
\end{tabular}


Table A.1 - continued from previous page

\begin{tabular}{|c|c|c|c|}
\hline ACC & $P_{N}$ & $B_{N}$ matrix row by row & $\mathrm{c} \#$ \\
\hline $37 \mathrm{I} 4 / \mathrm{mmm}$ & $4 m_{z}$ & $5\{\{1,0,0\},\{1,3,0\},\{0,3,6\}\}$ & \\
\hline $37 \mathrm{I} 4 / \mathrm{mmm}$ & $4 m_{z}$ & $6\left\{\{1,0,0\},\left\{1,2+c_{1}, 0\right\},\left\{0,0,2+c_{1}\right\}\right\}$ & \\
\hline $37 \mathrm{I} 4 / \mathrm{mmm}$ & $4 m_{z}$ & $7\left\{\{1,0,0\},\left\{1,4+c_{1}, 0\right\},\left\{0,4+c_{1}, 2\left(4+c_{1}\right)\right\}\right\}$ & \\
\hline $37 \mathrm{I} 4 / \mathrm{mmm}$ & $4 m_{z}$ & $8\left\{\{1,0,0\},\{1,2,0\},\left\{0,2\left(2+d_{1}\right), 2\left(3+d_{1}+d_{2}\right)\right\}\right\}$ & $24^{*}$ \\
\hline $37 \mathrm{I} 4 / \mathrm{mmm}$ & $4 m_{z}$ & $9\left\{\{1,0,0\},\{1,3,0\},\left\{0,3\left(1+d_{1}\right), 3\left(3+d_{1}+d_{2}\right)\right\}\right\}$ & $23^{*}$ \\
\hline $37 \mathrm{I} 4 / \mathrm{mmm}$ & $4 m_{z}$ & $10\left\{\{1,0,0\},\{1,3,0\},\left\{0,3\left(2+d_{1}\right), 3\left(3+d_{1}+d_{2}\right)\right\}\right\}$ & $24^{*}$ \\
\hline $37 \mathrm{I} 4 / \mathrm{mmm}$ & $4 m_{z}$ & $11\left\{\{1,0,0\},\left\{1,4+c_{1}, 0\right\},\left\{0,\left(4+c_{1}\right)\left(1+d_{1}\right),\left(4+c_{1}\right)\left(3+d_{1}+d_{2}\right)\right\}\right\}$ & $62^{*}$ \\
\hline $37 \mathrm{I} 4 / \mathrm{mmm}$ & $4 m_{z}$ & $12\left\{\{1,0,0\},\left\{1,4+c_{1}, 0\right\},\left\{0,\left(4+c_{1}\right)\left(2+d_{1}\right),\left(4+c_{1}\right)\left(3+d_{1}+d_{2}\right)\right\}\right\}$ & $63^{*}$ \\
\hline $37 \mathrm{I} 4 / \mathrm{mmm}$ & $52 / m_{z}$ & $1\{\{1,0,0\},\{0,1,0\},\{0,0,1\}\}$ & \\
\hline $37 \mathrm{I} 4 / \mathrm{mmm}$ & $52 / m_{z}$ & $2\{\{1,0,0\},\{1,2,0\},\{0,0,2\}\}$ & \\
\hline $37 \mathrm{I} 4 / \mathrm{mmm}$ & $52 / m_{z}$ & $3\{\{1,0,0\},\{0,1,0\},\{1,1,2\}\}$ & \\
\hline $37 \mathrm{I} 4 / \mathrm{mmm}$ & 6222 & $1\{\{1,0,0\},\{0,1,0\},\{3,3,4\}\}$ & \\
\hline $37 \mathrm{I} 4 / \mathrm{mmm}$ & 6222 & $2\{\{1,0,0\},\{0,1,0\},\{1,1,2\}\}$ & \\
\hline $37 \mathrm{I} 4 / \mathrm{mmm}$ & 6222 & $3\{\{1,0,0\},\{0,1,0\},\{0,0,1\}\}$ & \\
\hline $37 \mathrm{I} 4 / \mathrm{mmm}$ & 6222 & $4\{\{1,0,0\},\{0,1,0\},\{3,3,4\}\}$ & \\
\hline $37 \mathrm{I} 4 / \mathrm{mmm}$ & 6222 & $5\{\{1,0,0\},\{0,1,0\},\{1,1,2\}\}$ & \\
\hline $37 \mathrm{I} 4 / \mathrm{mmm}$ & 6222 & $6\{\{1,0,0\},\{0,1,0\},\{0,0,1\}\}$ & \\
\hline $37 \mathrm{I} 4 / \mathrm{mmm}$ & $7 m m 2_{z}$ & $1\{\{1,0,0\},\{0,1,0\},\{0,0,1\}\}$ & \\
\hline $37 \mathrm{I} 4 / \mathrm{mmm}$ & $7 m m 2_{z}$ & $2\left\{\{1,0,0\},\{0,1,0\},\left\{1,1,2+c_{1}\right\}\right\}$ & \\
\hline $37 \mathrm{I} 4 / \mathrm{mmm}$ & $8222_{d}$ & $1\{\{1,0,0\},\{0,1,0\},\{0,0,1\}\}$ & \\
\hline $37 \mathrm{I} 4 / \mathrm{mmm}$ & $8222_{d}$ & $2\{\{1,0,0\},\{1,2,0\},\{0,0,2\}\}$ & \\
\hline $37 \mathrm{I} 4 / \mathrm{mmm}$ & $8222_{d}$ & $3\{\{1,0,0\},\{0,1,0\},\{1,1,2\}\}$ & \\
\hline $37 \mathrm{I} 4 / \mathrm{mmm}$ & $9 m m 2_{d}$ & $1\{\{1,0,0\},\{1,2,0\},\{0,0,2\}\}$ & \\
\hline $37 \mathrm{I} 4 / \mathrm{mmm}$ & $9 m m 2_{d}$ & $2\{\{1,0,0\},\{0,1,0\},\{0,0,1\}\}$ & \\
\hline $37 \mathrm{I} 4 / \mathrm{mmm}$ & $9 m m 2_{d}$ & $3\left\{\{1,0,0\},\{1,2,0\},\left\{2,2,2\left(2+c_{1}\right)\right\}\right\}$ & \\
\hline $37 \mathrm{I} 4 / \mathrm{mmm}$ & $9 m m 2_{d}$ & $4\left\{\{1,0,0\},\{0,1,0\},\left\{3+2 c_{1}, 3+2 c_{1}, 4\left(1+c_{1}\right)\right\}\right\}$ & \\
\hline $37 \mathrm{I} 4 / \mathrm{mmm}$ & $9 m m 2_{d}$ & $5\left\{\{1,0,0\},\{0,1,0\},\left\{1,1,2+c_{1}\right\}\right\}$ & \\
\hline $37 \mathrm{I} 4 / \mathrm{mmm}$ & $10 \overline{4}$ & $1\{\{1,0,0\},\{0,1,0\},\{3,3,4\}\}$ & \\
\hline
\end{tabular}


Table A.1 - continued from previous page

\begin{tabular}{|c|c|c|c|}
\hline ACC & $P_{N}$ & $B_{N}$ matrix row by row & $\mathrm{c \#}$ \\
\hline $37 \mathrm{I} 4 / \mathrm{mmm}$ & $10 \overline{4}$ & $2\{\{1,0,0\},\{0,1,0\},\{1,1,2\}\}$ & \\
\hline $37 \mathrm{I} / \mathrm{mmm}$ & $10 \overline{4}$ & $3\{\{1,0,0\},\{0,1,0\},\{0,0,1\}\}$ & \\
\hline $37 \mathrm{I} / \mathrm{mmm}$ & $10 \overline{4}$ & $4\{\{1,0,0\},\{0,1,0\},\{3,3,4\}\}$ & \\
\hline $37 \mathrm{I} / \mathrm{mmm}$ & $10 \overline{4}$ & $5\{\{1,0,0\},\{0,1,0\},\{1,1,2\}\}$ & \\
\hline $37 \mathrm{I} 4 / \mathrm{mmm}$ & $10 \overline{4}$ & $6\{\{1,0,0\},\{0,1,0\},\{0,0,1\}\}$ & \\
\hline $37 \mathrm{I} 4 / \mathrm{mmm}$ & 114 & $1\{\{1,0,0\},\{0,1,0\},\{0,0,1\}\}$ & \\
\hline $37 \mathrm{I} 4 / \mathrm{mmm}$ & 114 & $2\left\{\{1,0,0\},\{0,1,0\},\left\{1,1,2+c_{1}\right\}\right\}$ & \\
\hline $37 \mathrm{I} 4 / \mathrm{mmm}$ & $12 \mathrm{mmm}$ & $1\{\{1,0,0\},\{0,1,0\},\{1,1,2\}\}$ & \\
\hline $37 \mathrm{I} 4 / \mathrm{mmm}$ & $12 \mathrm{mmm}$ & $2\{\{1,0,0\},\{0,1,0\},\{0,0,1\}\}$ & \\
\hline $37 \mathrm{I} 4 / \mathrm{mmm}$ & $13 \mathrm{mmm}_{d}$ & $1\{\{1,0,0\},\{0,1,0\},\{0,0,1\}\}$ & \\
\hline $37 \mathrm{I} 4 / \mathrm{mmm}$ & $13 \mathrm{mmm}_{d}$ & $2\{\{1,0,0\},\{1,2,0\},\{0,0,2\}\}$ & \\
\hline $37 \mathrm{I} 4 / \mathrm{mmm}$ & $13 \mathrm{mmm}_{d}$ & $3\{\{1,0,0\},\{0,1,0\},\{1,1,2\}\}$ & \\
\hline $37 \mathrm{I} 4 / \mathrm{mmm}$ & $144 / m$ & $1\{\{1,0,0\},\{0,1,0\},\{1,1,2\}\}$ & \\
\hline $37 \mathrm{I} 4 / \mathrm{mmm}$ & $144 / m$ & $2\{\{1,0,0\},\{0,1,0\},\{0,0,1\}\}$ & \\
\hline $37 \mathrm{I} 4 / \mathrm{mmm}$ & 15422 & $1\{\{1,0,0\},\{0,1,0\},\{1,1,2\}\}$ & \\
\hline $37 \mathrm{I} 4 / \mathrm{mmm}$ & 15422 & $2\{\{1,0,0\},\{0,1,0\},\{0,0,1\}\}$ & \\
\hline $37 \mathrm{I} 4 / \mathrm{mmm}$ & $16 \overline{4} 2 m$ & $1\{\{1,0,0\},\{0,1,0\},\{3,3,4\}\}$ & \\
\hline $37 \mathrm{I} 4 / \mathrm{mmm}$ & $16 \overline{4} 2 m$ & $2\{\{1,0,0\},\{0,1,0\},\{1,1,2\}\}$ & \\
\hline $37 \mathrm{I} 4 / \mathrm{mmm}$ & $16 \overline{4} 2 m$ & $3\{\{1,0,0\},\{0,1,0\},\{0,0,1\}\}$ & \\
\hline $37 \mathrm{I} 4 / \mathrm{mmm}$ & $16 \overline{4} 2 m$ & $4\{\{1,0,0\},\{0,1,0\},\{3,3,4\}\}$ & \\
\hline $37 \mathrm{I} 4 / \mathrm{mmm}$ & $16 \overline{4} 2 m$ & $5\{\{1,0,0\},\{0,1,0\},\{1,1,2\}\}$ & \\
\hline $37 \mathrm{I} 4 / \mathrm{mmm}$ & $16 \overline{4} 2 m$ & $6\{\{1,0,0\},\{0,1,0\},\{0,0,1\}\}$ & \\
\hline $37 \mathrm{I} / \mathrm{mmm}$ & $17 \overline{4} m 2$ & $1\{\{1,0,0\},\{0,1,0\},\{1,1,2\}\}$ & \\
\hline $37 \mathrm{I} 4 / \mathrm{mmm}$ & $17 \overline{4} m 2$ & $2\{\{1,0,0\},\{0,1,0\},\{0,0,1\}\}$ & \\
\hline $37 \mathrm{I} 4 / \mathrm{mmm}$ & $184 \mathrm{~mm}$ & $1\{\{1,0,0\},\{0,1,0\},\{0,0,1\}\}$ & \\
\hline $37 \mathrm{I} 4 / \mathrm{mmm}$ & $184 \mathrm{~mm}$ & $2\left\{\{1,0,0\},\{0,1,0\},\left\{1,1,2+c_{1}\right\}\right\}$ & \\
\hline $37 \mathrm{I} 4 / \mathrm{mmm}$ & $194 / \mathrm{mmm}$ & $1\{\{1,0,0\},\{0,1,0\},\{1,1,2\}\}$ & \\
\hline $37 \mathrm{I} 4 / \mathrm{mmm}$ & $194 / \mathrm{mmm}$ & $2\{\{1,0,0\},\{0,1,0\},\{0,0,1\}\}$ & \\
\hline
\end{tabular}


Table A.1 - continued from previous page

\begin{tabular}{|c|c|c|c|}
\hline ACC & $P_{N}$ & $B_{N}$ matrix row by row & $\mathrm{c \#}$ \\
\hline $38 P 3$ & 23 & $1\left\{\{1,0,0\},\{2,3,0\},\left\{0,0,1+c_{1}\right\}\right\}$ & \\
\hline $38 P 3$ & 23 & $2\left\{\{1,0,0\},\{0,1,0\},\left\{2+2 c_{1}, 2+2 c_{1}, 3+3 c_{1}\right\}\right\}$ & \\
\hline $38 P 3$ & 23 & $3\left\{\{1,0,0\},\{0,1,0\},\left\{1+c_{1}, 1+c_{1}, 3+3 c_{1}\right\}\right\}$ & \\
\hline $38 P 3$ & 23 & $4\left\{\{1,0,0\},\{0,1,0\},\left\{0,0,1+c_{1}\right\}\right\}$ & \\
\hline $39 R 3$ & 23 & $1\left\{\{1,0,0\},\{0,1,0\},\left\{c_{1}, c_{1}, 1+c_{1}\right\}\right\}$ & \\
\hline $40 P \overline{3}$ & $2 \overline{1}$ & $1\{\{1,0,0\},\{0,1,0\},\{0,0,1\}\}$ & \\
\hline $40 P \overline{3}$ & $2 \overline{1}$ & $2\{\{1,0,0\},\{0,1,0\},\{0,0,2\}\}$ & \\
\hline $40 P \overline{3}$ & $2 \overline{1}$ & $3\{\{2,0,0\},\{0,2,0\},\{0,0,1\}\}$ & \\
\hline $40 P \overline{3}$ & $2 \overline{1}$ & $4\{\{2,0,0\},\{0,2,0\},\{0,0,2\}\}$ & \\
\hline $40 P \overline{3}$ & 33 & $1\left\{\{1,0,0\},\{2,3,0\},\left\{0,0,1+c_{1}\right\}\right\}$ & \\
\hline $40 P \overline{3}$ & 33 & $2\left\{\{1,0,0\},\{0,1,0\},\left\{2+2 c_{1}, 2+2 c_{1}, 3+3 c_{1}\right\}\right\}$ & \\
\hline $40 P \overline{3}$ & 33 & $3\left\{\{1,0,0\},\{0,1,0\},\left\{1+c_{1}, 1+c_{1}, 3+3 c_{1}\right\}\right\}$ & \\
\hline $40 P \overline{3}$ & 33 & $4\left\{\{1,0,0\},\{0,1,0\},\left\{0,0,1+c_{1}\right\}\right\}$ & \\
\hline $40 P \overline{3}$ & $4 \overline{3}$ & $1\{\{1,0,0\},\{0,1,0\},\{0,0,1\}\}$ & \\
\hline $40 P \overline{3}$ & $4 \overline{3}$ & $2\{\{1,0,0\},\{0,1,0\},\{0,0,2\}\}$ & \\
\hline $41 R \overline{3}$ & $2 \overline{1}$ & $1\{\{1,0,0\},\{0,1,0\},\{0,0,1\}\}$ & \\
\hline $41 R \overline{3}$ & $2 \overline{1}$ & $2\{\{1,0,0\},\{0,1,0\},\{1,1,2\}\}$ & \\
\hline $41 R \overline{3}$ & $2 \overline{1}$ & $3\{\{1,0,0\},\{1,2,0\},\{1,0,2\}\}$ & \\
\hline $41 R \overline{3}$ & $2 \overline{1}$ & $4\{\{2,0,0\},\{0,2,0\},\{0,0,2\}\}$ & \\
\hline $41 R \overline{3}$ & 33 & $1\left\{\{1,0,0\},\{0,1,0\},\left\{c_{1}, c_{1}, 1+c_{1}\right\}\right\}$ & \\
\hline $41 R \overline{3}$ & $4 \overline{3}$ & $1\{\{1,0,0\},\{0,1,0\},\{1,1,2\}\}$ & \\
\hline $41 R \overline{3}$ & $4 \overline{3}$ & $2\{\{1,0,0\},\{0,1,0\},\{0,0,1\}\}$ & \\
\hline $42 P 312$ & 23 & $1\left\{\{1,0,0\},\{2,3,0\},\left\{0,0,1+c_{1}\right\}\right\}$ & \\
\hline $42 P 312$ & 23 & $2\left\{\{1,0,0\},\{0,1,0\},\left\{2+2 c_{1}, 2+2 c_{1}, 3+3 c_{1}\right\}\right\}$ & \\
\hline $42 P 312$ & 23 & $3\left\{\{1,0,0\},\{0,1,0\},\left\{1+c_{1}, 1+c_{1}, 3+3 c_{1}\right\}\right\}$ & \\
\hline $42 P 312$ & 23 & $4\left\{\{1,0,0\},\{0,1,0\},\left\{0,0,1+c_{1}\right\}\right\}$ & \\
\hline $42 P 312$ & 3312 & $1\{\{1,0,0\},\{0,1,0\},\{0,0,1\}\}$ & \\
\hline $42 P 312$ & 3312 & $2\{\{1,0,0\},\{0,1,0\},\{0,0,2\}\}$ & \\
\hline
\end{tabular}


Table A.1 - continued from previous page

\begin{tabular}{|c|c|c|c|}
\hline ACC & $P_{N}$ & $B_{N}$ matrix row by row & $\mathrm{c} \#$ \\
\hline $43 P 321$ & 23 & $1\left\{\{1,0,0\},\{2,3,0\},\left\{0,0,1+c_{1}\right\}\right\}$ & \\
\hline $43 P 321$ & 23 & $2\left\{\{1,0,0\},\{0,1,0\},\left\{0,0,1+c_{1}\right\}\right\}$ & \\
\hline $43 P 321$ & 3321 & $1\{\{1,0,0\},\{2,3,0\},\{0,0,1\}\}$ & \\
\hline $43 P 321$ & 3321 & $2\{\{1,0,0\},\{0,1,0\},\{0,0,1\}\}$ & \\
\hline $43 P 321$ & 3321 & $3\{\{1,0,0\},\{2,3,0\},\{0,0,2\}\}$ & \\
\hline $43 P 321$ & 3321 & $4\{\{1,0,0\},\{0,1,0\},\{0,0,2\}\}$ & \\
\hline $44 R 32$ & 23 & $1\left\{\{1,0,0\},\{0,1,0\},\left\{c_{1}, c_{1}, 1+c_{1}\right\}\right\}$ & \\
\hline $44 R 32$ & 3321 & $1\{\{1,0,0\},\{0,1,0\},\{1,1,2\}\}$ & \\
\hline $44 R 32$ & 3321 & $2\{\{1,0,0\},\{0,1,0\},\{0,0,1\}\}$ & \\
\hline $45 P 3 m 1$ & 23 & $1\left\{\{1,0,0\},\{2,3,0\},\left\{0,0,1+c_{1}\right\}\right\}$ & \\
\hline $45 P 3 m 1$ & 23 & $2\left\{\{1,0,0\},\{0,1,0\},\left\{0,0,1+c_{1}\right\}\right\}$ & \\
\hline $45 P 3 m 1$ & $33 m 1$ & $1\left\{\{1,0,0\},\{0,1,0\},\left\{0,0,1+c_{1}\right\}\right\}$ & \\
\hline $46 P 31 m$ & 23 & $1\left\{\{1,0,0\},\{2,3,0\},\left\{0,0,1+c_{1}\right\}\right\}$ & \\
\hline $46 P 31 m$ & 23 & $2\left\{\{1,0,0\},\{0,1,0\},\left\{2+2 c_{1}, 2+2 c_{1}, 3+3 c_{1}\right\}\right\}$ & \\
\hline $46 P 31 m$ & 23 & $3\left\{\{1,0,0\},\{0,1,0\},\left\{1+c_{1}, 1+c_{1}, 3+3 c_{1}\right\}\right\}$ & \\
\hline $46 P 31 m$ & 23 & $4\left\{\{1,0,0\},\{0,1,0\},\left\{0,0,1+c_{1}\right\}\right\}$ & \\
\hline $46 P 31 m$ & $331 m$ & $1\left\{\{1,0,0\},\{2,3,0\},\left\{0,0,1+c_{1}\right\}\right\}$ & \\
\hline $46 P 31 m$ & $331 m$ & $2\left\{\{1,0,0\},\{0,1,0\},\left\{2+2 c_{1}, 2+2 c_{1}, 3+3 c_{1}\right\}\right\}$ & \\
\hline $46 P 31 m$ & $331 m$ & $3\left\{\{1,0,0\},\{0,1,0\},\left\{1+c_{1}, 1+c_{1}, 3+3 c_{1}\right\}\right\}$ & \\
\hline $46 P 31 m$ & $331 m$ & $4\left\{\{1,0,0\},\{0,1,0\},\left\{0,0,1+c_{1}\right\}\right\}$ & \\
\hline $47 R 3 m$ & 23 & $1\left\{\{1,0,0\},\{0,1,0\},\left\{c_{1}, c_{1}, 1+c_{1}\right\}\right\}$ & \\
\hline $47 R 3 m$ & $33 m 1$ & $1\left\{\{1,0,0\},\{0,1,0\},\left\{c_{1}, c_{1}, 1+c_{1}\right\}\right\}$ & \\
\hline $48 P \overline{3} 1 m$ & $2 \overline{1}$ & $1\{\{1,0,0\},\{0,1,0\},\{0,0,1\}\}$ & \\
\hline $48 P \overline{3} 1 m$ & $2 \overline{1}$ & $2\{\{1,0,0\},\{0,1,0\},\{0,0,2\}\}$ & \\
\hline $48 P \overline{3} 1 m$ & $2 \overline{1}$ & $3\{\{2,0,0\},\{0,2,0\},\{0,0,1\}\}$ & \\
\hline $48 P \overline{3} 1 m$ & $2 \overline{1}$ & $4\{\{2,0,0\},\{0,2,0\},\{0,0,2\}\}$ & \\
\hline $48 P \overline{3} 1 m$ & 33 & $1\left\{\{1,0,0\},\{2,3,0\},\left\{0,0,1+c_{1}\right\}\right\}$ & \\
\hline $48 P \overline{3} 1 m$ & 33 & $2\left\{\{1,0,0\},\{0,1,0\},\left\{2+2 c_{1}, 2+2 c_{1}, 3+3 c_{1}\right\}\right\}$ & \\
\hline
\end{tabular}


Table A.1 - continued from previous page

\begin{tabular}{|c|c|c|c|}
\hline ACC & $P_{N}$ & $B_{N}$ matrix row by row & $\mathrm{c \#}$ \\
\hline $48 P \overline{3} 1 m$ & 33 & $3\left\{\{1,0,0\},\{0,1,0\},\left\{1+c_{1}, 1+c_{1}, 3+3 c_{1}\right\}\right\}$ & \\
\hline $48 P \overline{3} 1 m$ & 33 & $4\left\{\{1,0,0\},\{0,1,0\},\left\{0,0,1+c_{1}\right\}\right\}$ & \\
\hline $48 P \overline{3} 1 m$ & $4 \overline{3}$ & $1\{\{1,0,0\},\{0,1,0\},\{0,0,1\}\}$ & \\
\hline $48 P \overline{3} 1 \mathrm{~m}$ & $4 \overline{3}$ & $2\{\{1,0,0\},\{0,1,0\},\{0,0,2\}\}$ & \\
\hline $48 P \overline{3} 1 m$ & $531 m$ & $1\left\{\{1,0,0\},\{2,3,0\},\left\{0,0,1+c_{1}\right\}\right\}$ & \\
\hline $48 P \overline{3} 1 m$ & $531 m$ & $2\left\{\{1,0,0\},\{0,1,0\},\left\{2+2 c_{1}, 2+2 c_{1}, 3+3 c_{1}\right\}\right\}$ & \\
\hline $48 P \overline{3} 1 m$ & $531 \mathrm{~m}$ & $3\left\{\{1,0,0\},\{0,1,0\},\left\{1+c_{1}, 1+c_{1}, 3+3 c_{1}\right\}\right\}$ & \\
\hline $48 P \overline{3} 1 \mathrm{~m}$ & $531 \mathrm{~m}$ & $4\left\{\{1,0,0\},\{0,1,0\},\left\{0,0,1+c_{1}\right\}\right\}$ & \\
\hline $48 P \overline{3} 1 \mathrm{~m}$ & 6312 & $1\{\{1,0,0\},\{0,1,0\},\{0,0,1\}\}$ & \\
\hline $48 P \overline{3} 1 m$ & 6312 & $2\{\{1,0,0\},\{0,1,0\},\{0,0,2\}\}$ & \\
\hline $48 P \overline{3} 1 m$ & $7 \overline{3} 1 m$ & $1\{\{1,0,0\},\{0,1,0\},\{0,0,1\}\}$ & \\
\hline $48 P \overline{3} 1 m$ & $7 \overline{3} 1 m$ & $2\{\{1,0,0\},\{0,1,0\},\{0,0,2\}\}$ & \\
\hline $49 P \overline{3} m 1$ & $2 \overline{1}$ & $1\{\{1,0,0\},\{0,1,0\},\{0,0,1\}\}$ & \\
\hline $49 P \overline{3} m 1$ & $2 \overline{1}$ & $2\{\{1,0,0\},\{0,1,0\},\{0,0,2\}\}$ & \\
\hline $49 P \overline{3} m 1$ & $2 \overline{1}$ & $3\{\{2,0,0\},\{0,2,0\},\{0,0,1\}\}$ & \\
\hline $49 P \overline{3} m 1$ & $2 \overline{1}$ & $4\{\{2,0,0\},\{0,2,0\},\{0,0,2\}\}$ & \\
\hline $49 P \overline{3} m 1$ & 33 & $1\left\{\{1,0,0\},\{2,3,0\},\left\{0,0,1+c_{1}\right\}\right\}$ & \\
\hline $49 P \overline{3} m 1$ & 33 & $2\left\{\{1,0,0\},\{0,1,0\},\left\{0,0,1+c_{1}\right\}\right\}$ & \\
\hline $49 P \overline{3} m 1$ & $4 \overline{3}$ & $1\{\{1,0,0\},\{0,1,0\},\{0,0,1\}\}$ & \\
\hline $49 P \overline{3} m 1$ & $4 \overline{3}$ & $2\{\{1,0,0\},\{0,1,0\},\{0,0,2\}\}$ & \\
\hline $49 P \overline{3} m 1$ & 5321 & $1\{\{1,0,0\},\{2,3,0\},\{0,0,1\}\}$ & \\
\hline $49 P \overline{3} m 1$ & 5321 & $2\{\{1,0,0\},\{0,1,0\},\{0,0,1\}\}$ & \\
\hline $49 P \overline{3} m 1$ & 5321 & $3\{\{1,0,0\},\{2,3,0\},\{0,0,2\}\}$ & \\
\hline $49 P \overline{3} m 1$ & 5321 & $4\{\{1,0,0\},\{0,1,0\},\{0,0,2\}\}$ & \\
\hline $49 P \overline{3} m 1$ & $63 m 1$ & $1\left\{\{1,0,0\},\{0,1,0\},\left\{0,0,1+c_{1}\right\}\right\}$ & \\
\hline $49 P \overline{3} m 1$ & $7 \overline{3} m 1$ & $1\{\{1,0,0\},\{0,1,0\},\{0,0,1\}\}$ & \\
\hline $49 P \overline{3} m 1$ & $7 \overline{3} m 1$ & $2\{\{1,0,0\},\{0,1,0\},\{0,0,2\}\}$ & \\
\hline $50 R \overline{3} m$ & $2 \overline{1}$ & $1\{\{1,0,0\},\{0,1,0\},\{0,0,1\}\}$ & \\
\hline
\end{tabular}


Table A.1 - continued from previous page

\begin{tabular}{|c|c|c|c|}
\hline ACC & $P_{N}$ & $B_{N}$ matrix row by row & $\mathrm{c} \#$ \\
\hline $50 R \overline{3} m$ & $2 \overline{1}$ & $2\{\{1,0,0\},\{0,1,0\},\{1,1,2\}\}$ & \\
\hline $50 R \overline{3} m$ & $2 \overline{1}$ & $3\{\{1,0,0\},\{1,2,0\},\{1,0,2\}\}$ & \\
\hline $50 R \overline{3} m$ & $2 \overline{1}$ & $4\{\{2,0,0\},\{0,2,0\},\{0,0,2\}\}$ & \\
\hline $50 R \overline{3} m$ & 33 & $1\left\{\{1,0,0\},\{0,1,0\},\left\{c_{1}, c_{1}, 1+c_{1}\right\}\right\}$ & \\
\hline $50 R \overline{3} m$ & $4 \overline{3}$ & $1\{\{1,0,0\},\{0,1,0\},\{1,1,2\}\}$ & \\
\hline $50 R \overline{3} m$ & $4 \overline{3}$ & $2\{\{1,0,0\},\{0,1,0\},\{0,0,1\}\}$ & \\
\hline $50 R \overline{3} m$ & 5321 & $1\{\{1,0,0\},\{0,1,0\},\{1,1,2\}\}$ & \\
\hline $50 R \overline{3} m$ & 5321 & $2\{\{1,0,0\},\{0,1,0\},\{0,0,1\}\}$ & \\
\hline $50 R \overline{3} m$ & $63 m 1$ & $1\left\{\{1,0,0\},\{0,1,0\},\left\{c_{1}, c_{1}, 1+c_{1}\right\}\right\}$ & \\
\hline $50 R \overline{3} m$ & $7 \overline{3} m 1$ & $1\{\{1,0,0\},\{0,1,0\},\{1,1,2\}\}$ & \\
\hline $50 R \overline{3} m$ & $7 \overline{3} m 1$ & $2\{\{1,0,0\},\{0,1,0\},\{0,0,1\}\}$ & \\
\hline $51 P 6$ & $22_{z}$ & $1\left\{\{1,0,0\},\{0,1,0\},\left\{0,0,1+c_{1}\right\}\right\}$ & \\
\hline $51 P 6$ & $22_{z}$ & $2\left\{\{2,0,0\},\{0,2,0\},\left\{0,0,1+c_{1}\right\}\right\}$ & \\
\hline $51 P 6$ & 33 & $1\left\{\{1,0,0\},\{2,3,0\},\left\{0,0,1+c_{1}\right\}\right\}$ & \\
\hline $51 P 6$ & 33 & $2\left\{\{1,0,0\},\{0,1,0\},\left\{0,0,1+c_{1}\right\}\right\}$ & \\
\hline $51 P 6$ & 46 & $1\left\{\{1,0,0\},\{0,1,0\},\left\{0,0,1+c_{1}\right\}\right\}$ & \\
\hline $52 P \overline{6}$ & $2 m_{z}$ & $1\left\{\left\{1+c_{1}, 0,0\right\},\left\{0,1+c_{1}, 0\right\},\{0,0,1\}\right\}$ & \\
\hline $52 P \overline{6}$ & $2 m_{z}$ & $2\left\{\left\{1+c_{1}, 0,0\right\},\left\{\left(1+c_{1}\right)\left(1+d_{1}\right),\left(1+c_{1}\right)\left(2+d_{1}+d_{2}\right), 0\right\},\{0,0,1\}\right\}$ & 34 \\
\hline $52 P \overline{6}$ & $2 m_{z}$ & $3\left\{\left\{1+c_{1}, 0,0\right\},\left\{0,1+c_{1}, 0\right\},\{0,0,2\}\right\}$ & \\
\hline $52 P \overline{6}$ & $2 m_{z}$ & $4\left\{\left\{1+c_{1}, 0,0\right\},\left\{\left(1+c_{1}\right)\left(1+d_{1}\right),\left(1+c_{1}\right)\left(2+d_{1}+d_{2}\right), 0\right\},\{0,0,2\}\right\}$ & 34 \\
\hline $52 P \overline{6}$ & $2 m_{z}$ & $5\left\{\left\{1+c_{1}, 0,0\right\},\left\{\left(1+c_{1}\right)\left(1+2 d_{1}\right),\left(1+c_{1}\right)\left(3+2 d_{1}+d_{2}\right), 0\right\},\{0,1,2\}\right\}$ & $59^{*}$ \\
\hline $52 P \overline{6}$ & $2 m_{z}$ & $6\left\{\left\{1+c_{1}, 0,0\right\},\left\{2\left(1+c_{1}\right)\left(1+d_{1}\right), 2\left(1+c_{1}\right)\left(2+d_{1}+d_{2}\right), 0\right\},\{1,0,2\}\right\}$ & $36^{*}$ \\
\hline $52 P \overline{6}$ & $2 m_{z}$ & $7\left\{\left\{1+c_{1}, 0,0\right\},\left\{\left(1+c_{1}\right)\left(1+d_{1}\right),\left(1+c_{1}\right)\left(4+d_{1}+2 d_{2}\right), 0\right\},\{1,1,2\}\right\}$ & $65^{*}$ \\
\hline $52 P \overline{6}$ & 33 & $1\left\{\{1,0,0\},\{2,3,0\},\left\{0,0,1+c_{1}\right\}\right\}$ & \\
\hline $52 P \overline{6}$ & 33 & $2\left\{\{1,0,0\},\{0,1,0\},\left\{0,0,1+c_{1}\right\}\right\}$ & \\
\hline $52 P \overline{6}$ & $4 \overline{6}$ & $1\{\{1,0,0\},\{2,3,0\},\{0,0,1\}\}$ & \\
\hline $52 P \overline{6}$ & $4 \overline{6}$ & $2\{\{1,0,0\},\{0,1,0\},\{0,0,1\}\}$ & \\
\hline $52 P \overline{6}$ & $4 \overline{6}$ & $3\{\{1,0,0\},\{2,3,0\},\{0,0,2\}\}$ & \\
\hline
\end{tabular}


Table A.1 - continued from previous page

\begin{tabular}{|c|c|c|c|}
\hline ACC & $P_{N}$ & $B_{N}$ matrix row by row & $\mathrm{c} \#$ \\
\hline $52 P \overline{6}$ & $4 \overline{6}$ & $4\{\{1,0,0\},\{0,1,0\},\{0,0,2\}\}$ & \\
\hline $53 P 6 / m$ & $2 \overline{1}$ & $1\{\{1,0,0\},\{0,1,0\},\{0,0,1\}\}$ & \\
\hline $53 P 6 / m$ & $2 \overline{1}$ & $2\{\{1,0,0\},\{0,1,0\},\{0,0,2\}\}$ & \\
\hline $53 P 6 / m$ & $2 \overline{1}$ & $3\{\{2,0,0\},\{0,2,0\},\{0,0,1\}\}$ & \\
\hline $53 P 6 / m$ & $2 \overline{1}$ & $4\{\{2,0,0\},\{0,2,0\},\{0,0,2\}\}$ & \\
\hline $53 P 6 / m$ & $32 z$ & $1\left\{\{1,0,0\},\{0,1,0\},\left\{0,0,1+c_{1}\right\}\right\}$ & \\
\hline $53 P 6 / m$ & $32 z$ & $2\left\{\{2,0,0\},\{0,2,0\},\left\{0,0,1+c_{1}\right\}\right\}$ & \\
\hline $53 P 6 / m$ & $4 m_{z}$ & $1\left\{\left\{1+c_{1}, 0,0\right\},\left\{0,1+c_{1}, 0\right\},\{0,0,1\}\right\}$ & \\
\hline $53 P 6 / m$ & $4 m_{z}$ & $2\left\{\left\{1+c_{1}, 0,0\right\},\left\{\left(1+c_{1}\right)\left(1+d_{1}\right),\left(1+c_{1}\right)\left(2+d_{1}+d_{2}\right), 0\right\},\{0,0,1\}\right\}$ & 34 \\
\hline $53 P 6 / m$ & $4 m_{z}$ & $3\left\{\left\{1+c_{1}, 0,0\right\},\left\{0,1+c_{1}, 0\right\},\{0,0,2\}\right\}$ & \\
\hline $53 P 6 / m$ & $4 m_{z}$ & $4\left\{\left\{1+c_{1}, 0,0\right\},\left\{\left(1+c_{1}\right)\left(1+d_{1}\right),\left(1+c_{1}\right)\left(2+d_{1}+d_{2}\right), 0\right\},\{0,0,2\}\right\}$ & 34 \\
\hline $53 P 6 / m$ & $4 m_{z}$ & $5\left\{\left\{1+c_{1}, 0,0\right\},\left\{\left(1+c_{1}\right)\left(1+2 d_{1}\right),\left(1+c_{1}\right)\left(3+2 d_{1}+d_{2}\right), 0\right\},\{0,1,2\}\right\}$ & $59^{*}$ \\
\hline $53 P 6 / m$ & $4 m_{z}$ & $6\left\{\left\{1+c_{1}, 0,0\right\},\left\{2\left(1+c_{1}\right)\left(1+d_{1}\right), 2\left(1+c_{1}\right)\left(2+d_{1}+d_{2}\right), 0\right\},\{1,0,2\}\right\}$ & $36^{*}$ \\
\hline $53 P 6 / m$ & $4 m_{z}$ & $7\left\{\left\{1+c_{1}, 0,0\right\},\left\{\left(1+c_{1}\right)\left(1+d_{1}\right),\left(1+c_{1}\right)\left(4+d_{1}+2 d_{2}\right), 0\right\},\{1,1,2\}\right\}$ & $65^{*}$ \\
\hline $53 P 6 / m$ & 53 & $1\left\{\{1,0,0\},\{2,3,0\},\left\{0,0,1+c_{1}\right\}\right\}$ & \\
\hline $53 P 6 / m$ & 53 & $2\left\{\{1,0,0\},\{0,1,0\},\left\{0,0,1+c_{1}\right\}\right\}$ & \\
\hline $53 P 6 / m$ & $62 / m_{z}$ & $1\{\{1,0,0\},\{0,1,0\},\{0,0,1\}\}$ & \\
\hline $53 P 6 / m$ & $62 / m_{z}$ & $2\{\{1,0,0\},\{0,1,0\},\{0,0,2\}\}$ & \\
\hline $53 P 6 / m$ & $62 / m_{z}$ & $3\{\{2,0,0\},\{0,2,0\},\{0,0,1\}\}$ & \\
\hline $53 P 6 / m$ & $62 / m_{z}$ & $4\{\{2,0,0\},\{0,2,0\},\{0,0,2\}\}$ & \\
\hline $53 P 6 / m$ & $7 \overline{3}$ & $1\{\{1,0,0\},\{0,1,0\},\{0,0,1\}\}$ & \\
\hline $53 P 6 / m$ & $7 \overline{3}$ & $2\{\{1,0,0\},\{0,1,0\},\{0,0,2\}\}$ & \\
\hline $53 P 6 / m$ & 86 & $1\left\{\{1,0,0\},\{0,1,0\},\left\{0,0,1+c_{1}\right\}\right\}$ & \\
\hline $53 P 6 / m$ & $9 \overline{6}$ & $1\{\{1,0,0\},\{2,3,0\},\{0,0,1\}\}$ & \\
\hline $53 P 6 / m$ & $9 \overline{6}$ & $2\{\{1,0,0\},\{0,1,0\},\{0,0,1\}\}$ & \\
\hline $53 P 6 / m$ & $9 \overline{6}$ & $3\{\{1,0,0\},\{2,3,0\},\{0,0,2\}\}$ & \\
\hline $53 P 6 / m$ & $9 \overline{6}$ & $4\{\{1,0,0\},\{0,1,0\},\{0,0,2\}\}$ & \\
\hline $53 P 6 / m$ & $106 / m$ & $1\{\{1,0,0\},\{0,1,0\},\{0,0,1\}\}$ & \\
\hline
\end{tabular}


Table A.1 - continued from previous page

\begin{tabular}{|c|c|c|c|}
\hline ACC & $P_{N}$ & $B_{N}$ matrix row by row & $\mathrm{c \#}$ \\
\hline $53 P 6 / m$ & $106 / m$ & $2\{\{1,0,0\},\{0,1,0\},\{0,0,2\}\}$ & \\
\hline $54 P 622$ & $22 z$ & $1\left\{\{1,0,0\},\{0,1,0\},\left\{0,0,1+c_{1}\right\}\right\}$ & \\
\hline $54 P 622$ & $22 z$ & $2\left\{\{2,0,0\},\{0,2,0\},\left\{0,0,1+c_{1}\right\}\right\}$ & \\
\hline $54 P 622$ & 33 & $1\left\{\{1,0,0\},\{2,3,0\},\left\{0,0,1+c_{1}\right\}\right\}$ & \\
\hline $54 P 622$ & 33 & $2\left\{\{1,0,0\},\{0,1,0\},\left\{0,0,1+c_{1}\right\}\right\}$ & \\
\hline $54 P 622$ & 46 & $1\left\{\{1,0,0\},\{0,1,0\},\left\{0,0,1+c_{1}\right\}\right\}$ & \\
\hline $54 P 622$ & 5321 & $1\{\{1,0,0\},\{2,3,0\},\{0,0,1\}\}$ & \\
\hline $54 P 622$ & 5321 & $2\{\{1,0,0\},\{0,1,0\},\{0,0,1\}\}$ & \\
\hline $54 P 622$ & 5321 & $3\{\{1,0,0\},\{2,3,0\},\{0,0,2\}\}$ & \\
\hline $54 P 622$ & 5321 & $4\{\{1,0,0\},\{0,1,0\},\{0,0,2\}\}$ & \\
\hline $54 P 622$ & 6312 & $1\{\{1,0,0\},\{0,1,0\},\{0,0,1\}\}$ & \\
\hline $54 P 622$ & 6312 & $2\{\{1,0,0\},\{0,1,0\},\{0,0,2\}\}$ & \\
\hline $54 P 622$ & 7622 & $1\{\{1,0,0\},\{0,1,0\},\{0,0,1\}\}$ & \\
\hline $54 P 622$ & 7622 & $2\{\{1,0,0\},\{0,1,0\},\{0,0,2\}\}$ & \\
\hline 55 P6mm & $22 z$ & $1\left\{\{1,0,0\},\{0,1,0\},\left\{0,0,1+c_{1}\right\}\right\}$ & \\
\hline $55 P 6 \mathrm{~mm}$ & $22 z$ & $2\left\{\{2,0,0\},\{0,2,0\},\left\{0,0,1+c_{1}\right\}\right\}$ & \\
\hline $55 P 6 \mathrm{~mm}$ & 33 & $1\left\{\{1,0,0\},\{2,3,0\},\left\{0,0,1+c_{1}\right\}\right\}$ & \\
\hline 55 P6mm & 33 & $2\left\{\{1,0,0\},\{0,1,0\},\left\{0,0,1+c_{1}\right\}\right\}$ & \\
\hline $55 P 6 \mathrm{~mm}$ & 46 & $1\left\{\{1,0,0\},\{0,1,0\},\left\{0,0,1+c_{1}\right\}\right\}$ & \\
\hline $55 P 6 \mathrm{~mm}$ & $531 \mathrm{~m}$ & $1\left\{\{1,0,0\},\{2,3,0\},\left\{0,0,1+c_{1}\right\}\right\}$ & \\
\hline $55 P 6 \mathrm{~mm}$ & $531 \mathrm{~m}$ & $2\left\{\{1,0,0\},\{0,1,0\},\left\{0,0,1+c_{1}\right\}\right\}$ & \\
\hline $55 P 6 \mathrm{~mm}$ & $63 m 1$ & $1\left\{\{1,0,0\},\{0,1,0\},\left\{0,0,1+c_{1}\right\}\right\}$ & \\
\hline 55 P6mm & $76 \mathrm{~mm}$ & $1\left\{\{1,0,0\},\{0,1,0\},\left\{0,0,1+c_{1}\right\}\right\}$ & \\
\hline $56 P \overline{6} m 2$ & $2 m_{z}$ & $1\left\{\left\{1+c_{1}, 0,0\right\},\left\{0,1+c_{1}, 0\right\},\{0,0,1\}\right\}$ & \\
\hline $56 P \overline{6} m 2$ & $2 m_{z}$ & $2\left\{\left\{1+c_{1}, 0,0\right\},\left\{\left(1+c_{1}\right)\left(1+d_{1}\right),\left(1+c_{1}\right)\left(2+d_{1}+d_{2}\right), 0\right\},\{0,0,1\}\right\}$ & 61 \\
\hline $56 P \overline{6} m 2$ & $2 m_{z}$ & $3\left\{\left\{1+c_{1}, 0,0\right\},\left\{0,1+c_{1}, 0\right\},\{0,0,2\}\right\}$ & \\
\hline $56 P \overline{6} m 2$ & $2 m_{z}$ & $4\left\{\left\{1+c_{1}, 0,0\right\},\left\{\left(1+c_{1}\right)\left(1+d_{1}\right),\left(1+c_{1}\right)\left(2+d_{1}+d_{2}\right), 0\right\},\{0,0,2\}\right\}$ & 61 \\
\hline $56 P \overline{6} m 2$ & $2 m_{z}$ & $5\left\{\left\{1+c_{1}, 0,0\right\},\left\{2\left(1+c_{1}\right)\left(1+d_{1}\right), 2\left(1+c_{1}\right)\left(2+d_{1}+d_{2}\right), 0\right\},\{1,0,2\}\right\}$ & $60^{*}$ \\
\hline
\end{tabular}


Table A.1 - continued from previous page

\begin{tabular}{|c|c|c|c|}
\hline ACC & $P_{N}$ & $B_{N}$ matrix row by row & $\mathrm{c} \#$ \\
\hline $56 P \overline{6} m 2$ & 33 & $1\left\{\{1,0,0\},\{2,3,0\},\left\{0,0,1+c_{1}\right\}\right\}$ & \\
\hline $56 P \overline{6} m 2$ & 33 & $2\left\{\{1,0,0\},\{0,1,0\},\left\{0,0,1+c_{1}\right\}\right\}$ & \\
\hline $56 P \overline{6} m 2$ & $4 \overline{6}$ & $1\{\{1,0,0\},\{2,3,0\},\{0,0,1\}\}$ & \\
\hline $56 P \overline{6} m 2$ & $4 \overline{6}$ & $2\{\{1,0,0\},\{0,1,0\},\{0,0,1\}\}$ & \\
\hline $56 P \overline{6} m 2$ & $4 \overline{6}$ & $3\{\{1,0,0\},\{2,3,0\},\{0,0,2\}\}$ & \\
\hline $56 P \overline{6} m 2$ & $4 \overline{6}$ & $4\{\{1,0,0\},\{0,1,0\},\{0,0,2\}\}$ & \\
\hline $56 P \overline{6} m 2$ & 5312 & $1\{\{1,0,0\},\{0,1,0\},\{0,0,1\}\}$ & \\
\hline $56 P \overline{6} m 2$ & 5312 & $2\{\{1,0,0\},\{0,1,0\},\{0,0,2\}\}$ & \\
\hline $56 P \overline{6} m 2$ & $63 m 1$ & $1\left\{\{1,0,0\},\{0,1,0\},\left\{0,0,1+c_{1}\right\}\right\}$ & \\
\hline $56 P \overline{6} m 2$ & $7 \overline{6} m 2$ & $1\{\{1,0,0\},\{0,1,0\},\{0,0,1\}\}$ & \\
\hline $56 P \overline{6} m 2$ & $7 \overline{6} m 2$ & $2\{\{1,0,0\},\{0,1,0\},\{0,0,2\}\}$ & \\
\hline $57 P \overline{6} 2 m$ & $2 m_{z}$ & $1\left\{\left\{1+c_{1}, 0,0\right\},\left\{0,1+c_{1}, 0\right\},\{0,0,1\}\right\}$ & \\
\hline $57 P \overline{6} 2 m$ & $2 m_{z}$ & $2\left\{\left\{1+c_{1}, 0,0\right\},\left\{\left(1+c_{1}\right)\left(1+d_{1}\right),\left(1+c_{1}\right)\left(2+d_{1}+d_{2}\right), 0\right\},\{0,0,1\}\right\}$ & 61 \\
\hline $57 P \overline{6} 2 m$ & $2 m_{z}$ & $3\left\{\left\{1+c_{1}, 0,0\right\},\left\{0,1+c_{1}, 0\right\},\{0,0,2\}\right\}$ & \\
\hline $57 P \overline{6} 2 m$ & $2 m_{z}$ & $4\left\{\left\{1+c_{1}, 0,0\right\},\left\{\left(1+c_{1}\right)\left(1+d_{1}\right),\left(1+c_{1}\right)\left(2+d_{1}+d_{2}\right), 0\right\},\{0,0,2\}\right\}$ & 61 \\
\hline $57 P \overline{6} 2 m$ & $2 m_{z}$ & $5\left\{\left\{1+c_{1}, 0,0\right\},\left\{2\left(1+c_{1}\right)\left(1+d_{1}\right), 2\left(1+c_{1}\right)\left(2+d_{1}+d_{2}\right), 0\right\},\{1,0,2\}\right\}$ & $60^{*}$ \\
\hline $57 P \overline{6} 2 m$ & 33 & $1\left\{\{1,0,0\},\{2,3,0\},\left\{0,0,1+c_{1}\right\}\right\}$ & \\
\hline $57 P \overline{6} 2 m$ & 33 & $2\left\{\{1,0,0\},\{0,1,0\},\left\{0,0,1+c_{1}\right\}\right\}$ & \\
\hline $57 P \overline{6} 2 m$ & 4321 & $1\{\{1,0,0\},\{2,3,0\},\{0,0,1\}\}$ & \\
\hline $57 P \overline{6} 2 m$ & 4321 & $2\{\{1,0,0\},\{0,1,0\},\{0,0,1\}\}$ & \\
\hline $57 P \overline{6} 2 m$ & 4321 & $3\{\{1,0,0\},\{2,3,0\},\{0,0,2\}\}$ & \\
\hline $57 P \overline{6} 2 m$ & 4321 & $4\{\{1,0,0\},\{0,1,0\},\{0,0,2\}\}$ & \\
\hline $57 P \overline{6} 2 m$ & $531 m$ & $1\left\{\{1,0,0\},\{2,3,0\},\left\{0,0,1+c_{1}\right\}\right\}$ & \\
\hline $57 P \overline{6} 2 m$ & $531 m$ & $2\left\{\{1,0,0\},\{0,1,0\},\left\{0,0,1+c_{1}\right\}\right\}$ & \\
\hline $57 P \overline{6} 2 m$ & $6 \overline{6}$ & $1\{\{1,0,0\},\{2,3,0\},\{0,0,1\}\}$ & \\
\hline $57 P \overline{6} 2 m$ & $6 \overline{6}$ & $2\{\{1,0,0\},\{0,1,0\},\{0,0,1\}\}$ & \\
\hline $57 P \overline{6} 2 m$ & $6 \overline{6}$ & $3\{\{1,0,0\},\{2,3,0\},\{0,0,2\}\}$ & \\
\hline $57 P \overline{6} 2 m$ & $6 \overline{6}$ & $4\{\{1,0,0\},\{0,1,0\},\{0,0,2\}\}$ & \\
\hline
\end{tabular}


Table A.1 - continued from previous page

\begin{tabular}{|c|c|c|c|}
\hline ACC & $P_{N}$ & $B_{N}$ matrix row by row & $\mathrm{c} \#$ \\
\hline $57 P \overline{6} 2 m$ & $7 \overline{6} 2 m$ & $1\{\{1,0,0\},\{2,3,0\},\{0,0,1\}\}$ & \\
\hline $57 P \overline{6} 2 m$ & $7 \overline{6} 2 m$ & $2\{\{1,0,0\},\{0,1,0\},\{0,0,1\}\}$ & \\
\hline $57 P \overline{6} 2 m$ & $7 \overline{6} 2 m$ & $3\{\{1,0,0\},\{2,3,0\},\{0,0,2\}\}$ & \\
\hline $57 P \overline{6} 2 m$ & $7 \overline{6} 2 m$ & $4\{\{1,0,0\},\{0,1,0\},\{0,0,2\}\}$ & \\
\hline $58 \mathrm{P6} / \mathrm{mmm}$ & $2 \overline{1}$ & $1\{\{1,0,0\},\{0,1,0\},\{0,0,1\}\}$ & \\
\hline $58 \mathrm{P6} / \mathrm{mmm}$ & $2 \overline{1}$ & $2\{\{1,0,0\},\{0,1,0\},\{0,0,2\}\}$ & \\
\hline $58 \mathrm{P6} / \mathrm{mmm}$ & $2 \overline{1}$ & $3\{\{2,0,0\},\{0,2,0\},\{0,0,1\}\}$ & \\
\hline $58 \mathrm{P6} / \mathrm{mmm}$ & $2 \overline{1}$ & $4\{\{2,0,0\},\{0,2,0\},\{0,0,2\}\}$ & \\
\hline $58 \mathrm{P6} / \mathrm{mmm}$ & $32 z$ & $1\left\{\{1,0,0\},\{0,1,0\},\left\{0,0,1+c_{1}\right\}\right\}$ & \\
\hline $58 \mathrm{P6} / \mathrm{mmm}$ & $32 z$ & $2\left\{\{2,0,0\},\{0,2,0\},\left\{0,0,1+c_{1}\right\}\right\}$ & \\
\hline $58 \mathrm{P6} / \mathrm{mmm}$ & $4 m_{z}$ & $1\left\{\left\{1+c_{1}, 0,0\right\},\left\{0,1+c_{1}, 0\right\},\{0,0,1\}\right\}$ & \\
\hline $58 \mathrm{P} 6 / \mathrm{mmm}$ & $4 m_{z}$ & $2\left\{\left\{1+c_{1}, 0,0\right\},\left\{\left(1+c_{1}\right)\left(1+d_{1}\right),\left(1+c_{1}\right)\left(2+d_{1}+d_{2}\right), 0\right\},\{0,0,1\}\right\}$ & 61 \\
\hline $58 \mathrm{P6} / \mathrm{mmm}$ & $4 m_{z}$ & $3\left\{\left\{1+c_{1}, 0,0\right\},\left\{0,1+c_{1}, 0\right\},\{0,0,2\}\right\}$ & \\
\hline $58 \mathrm{P6} / \mathrm{mmm}$ & $4 m_{z}$ & $4\left\{\left\{1+c_{1}, 0,0\right\},\left\{\left(1+c_{1}\right)\left(1+d_{1}\right),\left(1+c_{1}\right)\left(2+d_{1}+d_{2}\right), 0\right\},\{0,0,2\}\right\}$ & 61 \\
\hline $58 \mathrm{P6} / \mathrm{mmm}$ & $4 m_{z}$ & $5\left\{\left\{1+c_{1}, 0,0\right\},\left\{2\left(1+c_{1}\right)\left(1+d_{1}\right), 2\left(1+c_{1}\right)\left(2+d_{1}+d_{2}\right), 0\right\},\{1,0,2\}\right\}$ & $60^{*}$ \\
\hline $58 \mathrm{P6} / \mathrm{mmm}$ & 53 & $1\left\{\{1,0,0\},\{2,3,0\},\left\{0,0,1+c_{1}\right\}\right\}$ & \\
\hline $58 \mathrm{P6} / \mathrm{mmm}$ & 53 & $2\left\{\{1,0,0\},\{0,1,0\},\left\{0,0,1+c_{1}\right\}\right\}$ & \\
\hline $58 \mathrm{P6} / \mathrm{mmm}$ & $62 / m_{z}$ & $1\{\{1,0,0\},\{0,1,0\},\{0,0,1\}\}$ & \\
\hline $58 \mathrm{P6} / \mathrm{mmm}$ & $62 / m_{z}$ & $2\{\{1,0,0\},\{0,1,0\},\{0,0,2\}\}$ & \\
\hline $58 \mathrm{P6} / \mathrm{mmm}$ & $62 / m_{z}$ & $3\{\{2,0,0\},\{0,2,0\},\{0,0,1\}\}$ & \\
\hline $58 \mathrm{P6} / \mathrm{mmm}$ & $62 / m_{z}$ & $4\{\{2,0,0\},\{0,2,0\},\{0,0,2\}\}$ & \\
\hline $58 \mathrm{P} 6 / \mathrm{mmm}$ & $7 \overline{3}$ & $1\{\{1,0,0\},\{0,1,0\},\{0,0,1\}\}$ & \\
\hline $58 \mathrm{P6} / \mathrm{mmm}$ & $7 \overline{3}$ & $2\{\{1,0,0\},\{0,1,0\},\{0,0,2\}\}$ & \\
\hline $58 \mathrm{P6} / \mathrm{mmm}$ & 86 & $1\left\{\{1,0,0\},\{0,1,0\},\left\{0,0,1+c_{1}\right\}\right\}$ & \\
\hline $58 \mathrm{P6} / \mathrm{mmm}$ & 9321 & $1\{\{1,0,0\},\{2,3,0\},\{0,0,1\}\}$ & \\
\hline $58 \mathrm{P6} / \mathrm{mmm}$ & 9321 & $2\{\{1,0,0\},\{0,1,0\},\{0,0,1\}\}$ & \\
\hline $58 \mathrm{P6} / \mathrm{mmm}$ & 9321 & $3\{\{1,0,0\},\{2,3,0\},\{0,0,2\}\}$ & \\
\hline $58 \mathrm{P6} / \mathrm{mmm}$ & 9321 & $4\{\{1,0,0\},\{0,1,0\},\{0,0,2\}\}$ & \\
\hline
\end{tabular}


Table A.1 - continued from previous page

\begin{tabular}{|c|c|c|c|}
\hline ACC & $P_{N}$ & $B_{N}$ matrix row by row & $\mathrm{c} \#$ \\
\hline $58 \mathrm{P6} / \mathrm{mmm}$ & $1031 m$ & $1\left\{\{1,0,0\},\{2,3,0\},\left\{0,0,1+c_{1}\right\}\right\}$ & \\
\hline $58 \mathrm{P6} / \mathrm{mmm}$ & $1031 \mathrm{~m}$ & $2\left\{\{1,0,0\},\{0,1,0\},\left\{0,0,1+c_{1}\right\}\right\}$ & \\
\hline $58 \mathrm{P6} / \mathrm{mmm}$ & $11 \overline{6}$ & $1\{\{1,0,0\},\{2,3,0\},\{0,0,1\}\}$ & \\
\hline $58 \mathrm{P6} / \mathrm{mmm}$ & $11 \overline{6}$ & $2\{\{1,0,0\},\{0,1,0\},\{0,0,1\}\}$ & \\
\hline $58 \mathrm{P6} / \mathrm{mmm}$ & $11 \overline{6}$ & $3\{\{1,0,0\},\{2,3,0\},\{0,0,2\}\}$ & \\
\hline $58 \mathrm{P6} / \mathrm{mmm}$ & $11 \overline{6}$ & $4\{\{1,0,0\},\{0,1,0\},\{0,0,2\}\}$ & \\
\hline $58 \mathrm{P6} / \mathrm{mmm}$ & 12312 & $1\{\{1,0,0\},\{0,1,0\},\{0,0,1\}\}$ & \\
\hline $58 \mathrm{P6} / \mathrm{mmm}$ & 12312 & $2\{\{1,0,0\},\{0,1,0\},\{0,0,2\}\}$ & \\
\hline $58 \mathrm{P6} / \mathrm{mmm}$ & $133 m 1$ & $1\left\{\{1,0,0\},\{0,1,0\},\left\{0,0,1+c_{1}\right\}\right\}$ & \\
\hline $58 \mathrm{P6} / \mathrm{mmm}$ & $146 / m$ & $1\{\{1,0,0\},\{0,1,0\},\{0,0,1\}\}$ & \\
\hline $58 \mathrm{P6} / \mathrm{mmm}$ & $146 / m$ & $2\{\{1,0,0\},\{0,1,0\},\{0,0,2\}\}$ & \\
\hline $58 \mathrm{P6} / \mathrm{mmm}$ & $15 \overline{3} m 1$ & $1\{\{1,0,0\},\{0,1,0\},\{0,0,1\}\}$ & \\
\hline $58 \mathrm{P6} / \mathrm{mmm}$ & $15 \overline{3} m 1$ & $2\{\{1,0,0\},\{0,1,0\},\{0,0,2\}\}$ & \\
\hline $58 \mathrm{P6} / \mathrm{mmm}$ & 16622 & $1\{\{1,0,0\},\{0,1,0\},\{0,0,1\}\}$ & \\
\hline $58 \mathrm{P6} / \mathrm{mmm}$ & 16622 & $2\{\{1,0,0\},\{0,1,0\},\{0,0,2\}\}$ & \\
\hline $58 \mathrm{P6} / \mathrm{mmm}$ & $17 \overline{6} 2 m$ & $1\{\{1,0,0\},\{2,3,0\},\{0,0,1\}\}$ & \\
\hline $58 \mathrm{P6} / \mathrm{mmm}$ & $17 \overline{6} 2 m$ & $2\{\{1,0,0\},\{0,1,0\},\{0,0,1\}\}$ & \\
\hline $58 \mathrm{P6} / \mathrm{mmm}$ & $17 \overline{6} 2 m$ & $3\{\{1,0,0\},\{2,3,0\},\{0,0,2\}\}$ & \\
\hline $58 \mathrm{P6} / \mathrm{mmm}$ & $17 \overline{6} 2 m$ & $4\{\{1,0,0\},\{0,1,0\},\{0,0,2\}\}$ & \\
\hline $58 \mathrm{P6} / \mathrm{mmm}$ & $18 \overline{3} 1 m$ & $1\{\{1,0,0\},\{0,1,0\},\{0,0,1\}\}$ & \\
\hline $58 \mathrm{P6} / \mathrm{mmm}$ & $18 \overline{3} 1 m$ & $2\{\{1,0,0\},\{0,1,0\},\{0,0,2\}\}$ & \\
\hline $58 \mathrm{P6} / \mathrm{mmm}$ & $196 \mathrm{~mm}$ & $1\left\{\{1,0,0\},\{0,1,0\},\left\{0,0,1+c_{1}\right\}\right\}$ & \\
\hline $58 \mathrm{P6} / \mathrm{mmm}$ & $20 \overline{6} m 2$ & $1\{\{1,0,0\},\{0,1,0\},\{0,0,1\}\}$ & \\
\hline $58 \mathrm{P6} / \mathrm{mmm}$ & $20 \overline{6} m 2$ & $2\{\{1,0,0\},\{0,1,0\},\{0,0,2\}\}$ & \\
\hline $58 \mathrm{P6} / \mathrm{mmm}$ & $216 / \mathrm{mmm}$ & $1\{\{1,0,0\},\{0,1,0\},\{0,0,1\}\}$ & \\
\hline $58 \mathrm{P6} / \mathrm{mmm}$ & $216 / \mathrm{mmm}$ & $2\{\{1,0,0\},\{0,1,0\},\{0,0,2\}\}$ & \\
\hline $59 P 23$ & 2222 & $1\{\{1,0,0\},\{0,1,0\},\{0,0,1\}\}$ & \\
\hline $59 P 23$ & 2222 & $2\{\{1,0,0\},\{0,1,0\},\{1,1,2\}\}$ & \\
\hline
\end{tabular}


Table A.1 - continued from previous page

\begin{tabular}{|c|c|c|c|}
\hline ACC & $P_{N}$ & $B_{N}$ matrix row by row & $\mathrm{c \#}$ \\
\hline $59 P 23$ & 2222 & $3\{\{1,0,0\},\{1,2,0\},\{1,0,2\}\}$ & \\
\hline $59 P 23$ & 2222 & $4\{\{2,0,0\},\{0,2,0\},\{0,0,2\}\}$ & \\
\hline $59 P 23$ & 323 & $1\{\{1,0,0\},\{0,1,0\},\{1,1,2\}\}$ & \\
\hline $59 P 23$ & 323 & $2\{\{1,0,0\},\{0,1,0\},\{0,0,1\}\}$ & \\
\hline $60 F 23$ & 2222 & $1\{\{1,0,0\},\{0,1,0\},\{0,0,1\}\}$ & \\
\hline $60 F 23$ & 2222 & $2\{\{1,0,0\},\{1,2,0\},\{1,0,2\}\}$ & \\
\hline $60 F 23$ & 323 & $1\{\{1,0,0\},\{0,1,0\},\{0,0,1\}\}$ & \\
\hline $61 I 23$ & 2222 & $1\{\{1,0,0\},\{0,1,0\},\{3,3,4\}\}$ & \\
\hline $61 I 23$ & 2222 & $2\{\{1,0,0\},\{0,1,0\},\{1,1,2\}\}$ & \\
\hline $61 I 23$ & 2222 & $3\{\{1,0,0\},\{0,1,0\},\{0,0,1\}\}$ & \\
\hline $61 I 23$ & 2222 & $4\{\{1,0,0\},\{0,1,0\},\{3,3,4\}\}$ & \\
\hline $61 I 23$ & 2222 & $5\{\{1,0,0\},\{0,1,0\},\{1,1,2\}\}$ & \\
\hline $61 I 23$ & 2222 & $6\{\{1,0,0\},\{0,1,0\},\{0,0,1\}\}$ & \\
\hline $61 I 23$ & 323 & $1\{\{1,0,0\},\{0,1,0\},\{3,3,4\}\}$ & \\
\hline $61 I 23$ & 323 & $2\{\{1,0,0\},\{0,1,0\},\{1,1,2\}\}$ & \\
\hline $61 I 23$ & 323 & $3\{\{1,0,0\},\{0,1,0\},\{0,0,1\}\}$ & \\
\hline $61 I 23$ & 323 & $4\{\{1,0,0\},\{0,1,0\},\{3,3,4\}\}$ & \\
\hline $61 I 23$ & 323 & $5\{\{1,0,0\},\{0,1,0\},\{1,1,2\}\}$ & \\
\hline $61 I 23$ & 323 & $6\{\{1,0,0\},\{0,1,0\},\{0,0,1\}\}$ & \\
\hline $62 P m \overline{3}$ & $2 \overline{1}$ & $1\{\{1,0,0\},\{0,1,0\},\{0,0,1\}\}$ & \\
\hline $62 P m \overline{3}$ & $2 \overline{1}$ & $2\{\{1,0,0\},\{0,1,0\},\{1,1,2\}\}$ & \\
\hline $62 P m \overline{3}$ & $2 \overline{1}$ & $3\{\{1,0,0\},\{1,2,0\},\{1,0,2\}\}$ & \\
\hline $62 P m \overline{3}$ & $2 \overline{1}$ & $4\{\{2,0,0\},\{0,2,0\},\{0,0,2\}\}$ & \\
\hline $62 P m \overline{3}$ & 3222 & $1\{\{1,0,0\},\{0,1,0\},\{0,0,1\}\}$ & \\
\hline $62 P m \overline{3}$ & 3222 & $2\{\{1,0,0\},\{0,1,0\},\{1,1,2\}\}$ & \\
\hline $62 P m \overline{3}$ & 3222 & $3\{\{1,0,0\},\{1,2,0\},\{1,0,2\}\}$ & \\
\hline $62 P m \overline{3}$ & 3222 & $4\{\{2,0,0\},\{0,2,0\},\{0,0,2\}\}$ & \\
\hline $62 P m \overline{3}$ & $4 \mathrm{mmm}$ & $1\{\{1,0,0\},\{0,1,0\},\{0,0,1\}\}$ & \\
\hline
\end{tabular}


Table A.1 - continued from previous page

\begin{tabular}{|c|c|c|c|}
\hline ACC & $P_{N}$ & $B_{N}$ matrix row by row & $\mathrm{c \#}$ \\
\hline $62 P m \overline{3}$ & $4 \mathrm{mmm}$ & $2\{\{1,0,0\},\{0,1,0\},\{1,1,2\}\}$ & \\
\hline $62 P m \overline{3}$ & $4 \mathrm{mmm}$ & $3\{\{1,0,0\},\{1,2,0\},\{1,0,2\}\}$ & \\
\hline $62 P m \overline{3}$ & $4 \mathrm{mmm}$ & $4\{\{2,0,0\},\{0,2,0\},\{0,0,2\}\}$ & \\
\hline $62 P m \overline{3}$ & 523 & $1\{\{1,0,0\},\{0,1,0\},\{1,1,2\}\}$ & \\
\hline $62 P m \overline{3}$ & 523 & $2\{\{1,0,0\},\{0,1,0\},\{0,0,1\}\}$ & \\
\hline $62 P m \overline{3}$ & $6 m \overline{3}$ & $1\{\{1,0,0\},\{0,1,0\},\{1,1,2\}\}$ & \\
\hline $62 P m \overline{3}$ & $6 m \overline{3}$ & $2\{\{1,0,0\},\{0,1,0\},\{0,0,1\}\}$ & \\
\hline $63 F m \overline{3}$ & $2 \overline{1}$ & $1\{\{1,0,0\},\{0,1,0\},\{0,0,1\}\}$ & \\
\hline $63 F m \overline{3}$ & $2 \overline{1}$ & $2\{\{1,0,0\},\{1,2,0\},\{1,0,2\}\}$ & \\
\hline $63 F m \overline{3}$ & $2 \overline{1}$ & $3\{\{2,0,0\},\{0,2,0\},\{0,0,2\}\}$ & \\
\hline $63 F m \overline{3}$ & 3222 & $1\{\{1,0,0\},\{0,1,0\},\{0,0,1\}\}$ & \\
\hline $63 F m \overline{3}$ & 3222 & $2\{\{1,0,0\},\{1,2,0\},\{1,0,2\}\}$ & \\
\hline $63 F m \overline{3}$ & $4 \mathrm{mmm}$ & $1\{\{1,0,0\},\{0,1,0\},\{0,0,1\}\}$ & \\
\hline $63 F m \overline{3}$ & $4 \mathrm{mmm}$ & $2\{\{1,0,0\},\{1,2,0\},\{1,0,2\}\}$ & \\
\hline $63 F m \overline{3}$ & 523 & $1\{\{1,0,0\},\{0,1,0\},\{0,0,1\}\}$ & \\
\hline $63 F m \overline{3}$ & $6 m \overline{3}$ & $1\{\{1,0,0\},\{0,1,0\},\{0,0,1\}\}$ & \\
\hline $64 \operatorname{Im} \overline{3}$ & $2 \overline{1}$ & $1\{\{1,0,0\},\{0,1,0\},\{0,0,1\}\}$ & \\
\hline $64 \operatorname{Im} \overline{3}$ & $2 \overline{1}$ & $2\{\{1,0,0\},\{0,1,0\},\{1,1,2\}\}$ & \\
\hline $64 \operatorname{Im} \overline{3}$ & $2 \overline{1}$ & $3\{\{2,0,0\},\{0,2,0\},\{0,0,2\}\}$ & \\
\hline $64 \operatorname{Im} \overline{3}$ & 3222 & $1\{\{1,0,0\},\{0,1,0\},\{3,3,4\}\}$ & \\
\hline $64 \operatorname{Im} \overline{3}$ & 3222 & $2\{\{1,0,0\},\{0,1,0\},\{1,1,2\}\}$ & \\
\hline $64 \operatorname{Im} \overline{3}$ & 3222 & $3\{\{1,0,0\},\{0,1,0\},\{0,0,1\}\}$ & \\
\hline $64 \operatorname{Im} \overline{3}$ & 3222 & $4\{\{1,0,0\},\{0,1,0\},\{3,3,4\}\}$ & \\
\hline $64 \operatorname{Im} \overline{3}$ & 3222 & $5\{\{1,0,0\},\{0,1,0\},\{1,1,2\}\}$ & \\
\hline $64 \operatorname{Im} \overline{3}$ & 3222 & $6\{\{1,0,0\},\{0,1,0\},\{0,0,1\}\}$ & \\
\hline $64 \operatorname{Im} \overline{3}$ & $4 \mathrm{mmm}$ & $1\{\{1,0,0\},\{0,1,0\},\{1,1,2\}\}$ & \\
\hline $64 \operatorname{Im} \overline{3}$ & $4 \mathrm{mmm}$ & $2\{\{1,0,0\},\{0,1,0\},\{0,0,1\}\}$ & \\
\hline $64 \operatorname{Im} \overline{3}$ & 523 & $1\{\{1,0,0\},\{0,1,0\},\{3,3,4\}\}$ & \\
\hline
\end{tabular}


Table A.1 - continued from previous page

\begin{tabular}{|c|c|c|c|}
\hline ACC & $P_{N}$ & $B_{N}$ matrix row by row & $\mathrm{c \#}$ \\
\hline $64 \operatorname{Im} \overline{3}$ & 523 & $2\{\{1,0,0\},\{0,1,0\},\{1,1,2\}\}$ & \\
\hline $64 \operatorname{Im} \overline{3}$ & 523 & $3\{\{1,0,0\},\{0,1,0\},\{0,0,1\}\}$ & \\
\hline $64 \operatorname{Im} \overline{3}$ & 523 & $4\{\{1,0,0\},\{0,1,0\},\{3,3,4\}\}$ & \\
\hline $64 \operatorname{Im} \overline{3}$ & 523 & $5\{\{1,0,0\},\{0,1,0\},\{1,1,2\}\}$ & \\
\hline $64 \operatorname{Im} \overline{3}$ & 523 & $6\{\{1,0,0\},\{0,1,0\},\{0,0,1\}\}$ & \\
\hline $64 \operatorname{Im} \overline{3}$ & $6 m \overline{3}$ & $1\{\{1,0,0\},\{0,1,0\},\{1,1,2\}\}$ & \\
\hline $64 \operatorname{Im} \overline{3}$ & $6 m \overline{3}$ & $2\{\{1,0,0\},\{0,1,0\},\{0,0,1\}\}$ & \\
\hline $65 P 432$ & 2222 & $1\{\{1,0,0\},\{0,1,0\},\{0,0,1\}\}$ & \\
\hline $65 P 432$ & 2222 & $2\{\{1,0,0\},\{0,1,0\},\{1,1,2\}\}$ & \\
\hline $65 P 432$ & 2222 & $3\{\{1,0,0\},\{1,2,0\},\{1,0,2\}\}$ & \\
\hline $65 P 432$ & 2222 & $4\{\{2,0,0\},\{0,2,0\},\{0,0,2\}\}$ & \\
\hline $65 P 432$ & 323 & $1\{\{1,0,0\},\{0,1,0\},\{1,1,2\}\}$ & \\
\hline $65 P 432$ & 323 & $2\{\{1,0,0\},\{0,1,0\},\{0,0,1\}\}$ & \\
\hline $65 P 432$ & 4432 & $1\{\{1,0,0\},\{0,1,0\},\{1,1,2\}\}$ & \\
\hline $65 P 432$ & 4432 & $2\{\{1,0,0\},\{0,1,0\},\{0,0,1\}\}$ & \\
\hline $66 F 432$ & 2222 & $1\{\{1,0,0\},\{0,1,0\},\{0,0,1\}\}$ & \\
\hline $66 F 432$ & 2222 & $2\{\{1,0,0\},\{1,2,0\},\{1,0,2\}\}$ & \\
\hline $66 F 432$ & 323 & $1\{\{1,0,0\},\{0,1,0\},\{0,0,1\}\}$ & \\
\hline $66 F 432$ & 4432 & $1\{\{1,0,0\},\{0,1,0\},\{0,0,1\}\}$ & \\
\hline $67 I 432$ & 2222 & $1\{\{1,0,0\},\{0,1,0\},\{3,3,4\}\}$ & \\
\hline $67 I 432$ & 2222 & $2\{\{1,0,0\},\{0,1,0\},\{1,1,2\}\}$ & \\
\hline $67 I 432$ & 2222 & $3\{\{1,0,0\},\{0,1,0\},\{0,0,1\}\}$ & \\
\hline $67 I 432$ & 2222 & $4\{\{1,0,0\},\{0,1,0\},\{3,3,4\}\}$ & \\
\hline $67 I 432$ & 2222 & $5\{\{1,0,0\},\{0,1,0\},\{1,1,2\}\}$ & \\
\hline $67 I 432$ & 2222 & $6\{\{1,0,0\},\{0,1,0\},\{0,0,1\}\}$ & \\
\hline $67 I 432$ & 323 & $1\{\{1,0,0\},\{0,1,0\},\{3,3,4\}\}$ & \\
\hline $67 I 432$ & 323 & $2\{\{1,0,0\},\{0,1,0\},\{1,1,2\}\}$ & \\
\hline $67 I 432$ & 323 & $3\{\{1,0,0\},\{0,1,0\},\{0,0,1\}\}$ & \\
\hline
\end{tabular}


Table A.1 - continued from previous page

\begin{tabular}{|c|c|c|c|}
\hline ACC & $P_{N}$ & $B_{N}$ matrix row by row & $\mathrm{c \#}$ \\
\hline $67 I 432$ & 323 & $4\{\{1,0,0\},\{0,1,0\},\{3,3,4\}\}$ & \\
\hline $67 I 432$ & 323 & $5\{\{1,0,0\},\{0,1,0\},\{1,1,2\}\}$ & \\
\hline $67 I 432$ & 323 & $6\{\{1,0,0\},\{0,1,0\},\{0,0,1\}\}$ & \\
\hline $67 I 432$ & 4432 & $1\{\{1,0,0\},\{0,1,0\},\{1,1,2\}\}$ & \\
\hline $67 I 432$ & 4432 & $2\{\{1,0,0\},\{0,1,0\},\{0,0,1\}\}$ & \\
\hline $68 P \overline{4} 3 m$ & 2222 & $1\{\{1,0,0\},\{0,1,0\},\{0,0,1\}\}$ & \\
\hline $68 P \overline{4} 3 m$ & 2222 & $2\{\{1,0,0\},\{0,1,0\},\{1,1,2\}\}$ & \\
\hline $68 P \overline{4} 3 m$ & 2222 & $3\{\{1,0,0\},\{1,2,0\},\{1,0,2\}\}$ & \\
\hline $68 P \overline{4} 3 m$ & 2222 & $4\{\{2,0,0\},\{0,2,0\},\{0,0,2\}\}$ & \\
\hline $68 P \overline{4} 3 m$ & 323 & $1\{\{1,0,0\},\{0,1,0\},\{1,1,2\}\}$ & \\
\hline $68 P \overline{4} 3 m$ & 323 & $2\{\{1,0,0\},\{0,1,0\},\{0,0,1\}\}$ & \\
\hline $68 P \overline{4} 3 m$ & $4 \overline{4} 3 m$ & $1\{\{1,0,0\},\{0,1,0\},\{1,1,2\}\}$ & \\
\hline $68 P \overline{4} 3 m$ & $4 \overline{4} 3 m$ & $2\{\{1,0,0\},\{0,1,0\},\{0,0,1\}\}$ & \\
\hline $69 F \overline{4} 3 m$ & 2222 & $1\{\{1,0,0\},\{0,1,0\},\{0,0,1\}\}$ & \\
\hline $69 F \overline{4} 3 m$ & 2222 & $2\{\{1,0,0\},\{1,2,0\},\{1,0,2\}\}$ & \\
\hline $69 F \overline{4} 3 m$ & 323 & $1\{\{1,0,0\},\{0,1,0\},\{0,0,1\}\}$ & \\
\hline $69 F \overline{4} 3 m$ & $4 \overline{4} 3 m$ & $1\{\{1,0,0\},\{0,1,0\},\{0,0,1\}\}$ & \\
\hline $70 I \overline{4} 3 m$ & 2222 & $1\{\{1,0,0\},\{0,1,0\},\{3,3,4\}\}$ & \\
\hline $70 I \overline{4} 3 m$ & 2222 & $2\{\{1,0,0\},\{0,1,0\},\{1,1,2\}\}$ & \\
\hline $70 I \overline{4} 3 m$ & 2222 & $3\{\{1,0,0\},\{0,1,0\},\{0,0,1\}\}$ & \\
\hline $70 I \overline{4} 3 m$ & 2222 & $4\{\{1,0,0\},\{0,1,0\},\{3,3,4\}\}$ & \\
\hline $70 I \overline{4} 3 m$ & 2222 & $5\{\{1,0,0\},\{0,1,0\},\{1,1,2\}\}$ & \\
\hline $70 I \overline{4} 3 m$ & 2222 & $6\{\{1,0,0\},\{0,1,0\},\{0,0,1\}\}$ & \\
\hline $70 I \overline{4} 3 m$ & 323 & $1\{\{1,0,0\},\{0,1,0\},\{3,3,4\}\}$ & \\
\hline $70 I \overline{4} 3 m$ & 323 & $2\{\{1,0,0\},\{0,1,0\},\{1,1,2\}\}$ & \\
\hline $70 I \overline{4} 3 m$ & 323 & $3\{\{1,0,0\},\{0,1,0\},\{0,0,1\}\}$ & \\
\hline $70 I \overline{4} 3 m$ & 323 & $4\{\{1,0,0\},\{0,1,0\},\{3,3,4\}\}$ & \\
\hline $70 I \overline{4} 3 m$ & 323 & $5\{\{1,0,0\},\{0,1,0\},\{1,1,2\}\}$ & \\
\hline
\end{tabular}


Table A.1 - continued from previous page

\begin{tabular}{|c|c|c|c|}
\hline ACC & $P_{N}$ & $B_{N}$ matrix row by row & $\mathrm{c \#}$ \\
\hline $70 I \overline{4} 3 m$ & 323 & $6\{\{1,0,0\},\{0,1,0\},\{0,0,1\}\}$ & \\
\hline $70 I \overline{4} 3 m$ & $4 \overline{4} 3 m$ & $1\{\{1,0,0\},\{0,1,0\},\{3,3,4\}\}$ & \\
\hline $70 I \overline{4} 3 m$ & $4 \overline{4} 3 m$ & $2\{\{1,0,0\},\{0,1,0\},\{1,1,2\}\}$ & \\
\hline $70 I \overline{4} 3 m$ & $4 \overline{4} 3 m$ & $3\{\{1,0,0\},\{0,1,0\},\{0,0,1\}\}$ & \\
\hline $70 I \overline{4} 3 m$ & $4 \overline{4} 3 m$ & $4\{\{1,0,0\},\{0,1,0\},\{3,3,4\}\}$ & \\
\hline $70 I \overline{4} 3 m$ & $4 \overline{4} 3 m$ & $5\{\{1,0,0\},\{0,1,0\},\{1,1,2\}\}$ & \\
\hline $70 I \overline{4} 3 m$ & $4 \overline{4} 3 m$ & $6\{\{1,0,0\},\{0,1,0\},\{0,0,1\}\}$ & \\
\hline $71 P m \overline{3} m$ & $2 \overline{1}$ & $1\{\{1,0,0\},\{0,1,0\},\{0,0,1\}\}$ & \\
\hline $71 P m \overline{3} m$ & $2 \overline{1}$ & $2\{\{1,0,0\},\{0,1,0\},\{1,1,2\}\}$ & \\
\hline $71 P m \overline{3} m$ & $2 \overline{1}$ & $3\{\{1,0,0\},\{1,2,0\},\{1,0,2\}\}$ & \\
\hline $71 P m \overline{3} m$ & $2 \overline{1}$ & $4\{\{2,0,0\},\{0,2,0\},\{0,0,2\}\}$ & \\
\hline $71 P m \overline{3} m$ & 3222 & $1\{\{1,0,0\},\{0,1,0\},\{0,0,1\}\}$ & \\
\hline $71 P m \overline{3} m$ & 3222 & $2\{\{1,0,0\},\{0,1,0\},\{1,1,2\}\}$ & \\
\hline $71 P m \overline{3} m$ & 3222 & $3\{\{1,0,0\},\{1,2,0\},\{1,0,2\}\}$ & \\
\hline $71 P m \overline{3} m$ & 3222 & $4\{\{2,0,0\},\{0,2,0\},\{0,0,2\}\}$ & \\
\hline $71 P m \overline{3} m$ & $4 \mathrm{mmm}$ & $1\{\{1,0,0\},\{0,1,0\},\{0,0,1\}\}$ & \\
\hline $71 P m \overline{3} m$ & $4 \mathrm{mmm}$ & $2\{\{1,0,0\},\{0,1,0\},\{1,1,2\}\}$ & \\
\hline $71 P m \overline{3} m$ & $4 \mathrm{mmm}$ & $3\{\{1,0,0\},\{1,2,0\},\{1,0,2\}\}$ & \\
\hline $71 P m \overline{3} m$ & $4 \mathrm{mmm}$ & $4\{\{2,0,0\},\{0,2,0\},\{0,0,2\}\}$ & \\
\hline $71 P m \overline{3} m$ & 523 & $1\{\{1,0,0\},\{0,1,0\},\{1,1,2\}\}$ & \\
\hline $71 P m \overline{3} m$ & 523 & $2\{\{1,0,0\},\{0,1,0\},\{0,0,1\}\}$ & \\
\hline $71 P m \overline{3} m$ & $6 m \overline{3}$ & $1\{\{1,0,0\},\{0,1,0\},\{1,1,2\}\}$ & \\
\hline $71 P m \overline{3} m$ & $6 m \overline{3}$ & $2\{\{1,0,0\},\{0,1,0\},\{0,0,1\}\}$ & \\
\hline $71 P m \overline{3} m$ & 7432 & $1\{\{1,0,0\},\{0,1,0\},\{1,1,2\}\}$ & \\
\hline $71 P m \overline{3} m$ & 7432 & $2\{\{1,0,0\},\{0,1,0\},\{0,0,1\}\}$ & \\
\hline $71 P m \overline{3} m$ & $8 \overline{4} 3 m$ & $1\{\{1,0,0\},\{0,1,0\},\{1,1,2\}\}$ & \\
\hline $71 P m \overline{3} m$ & $8 \overline{4} 3 m$ & $2\{\{1,0,0\},\{0,1,0\},\{0,0,1\}\}$ & \\
\hline $71 P m \overline{3} m$ & $9 m \overline{3} m$ & $1\{\{1,0,0\},\{0,1,0\},\{1,1,2\}\}$ & \\
\hline
\end{tabular}


Table A.1 - continued from previous page

\begin{tabular}{|c|c|c|c|}
\hline ACC & $P_{N}$ & $B_{N}$ matrix row by row & $\mathrm{c \#}$ \\
\hline $71 P m \overline{3} m$ & $9 m \overline{3} m$ & $2\{\{1,0,0\},\{0,1,0\},\{0,0,1\}\}$ & \\
\hline $72 F m \overline{3} m$ & $2 \overline{1}$ & $1\{\{1,0,0\},\{0,1,0\},\{0,0,1\}\}$ & \\
\hline $72 F m \overline{3} m$ & $2 \overline{1}$ & $2\{\{1,0,0\},\{1,2,0\},\{1,0,2\}\}$ & \\
\hline $72 F m \overline{3} m$ & $2 \overline{1}$ & $3\{\{2,0,0\},\{0,2,0\},\{0,0,2\}\}$ & \\
\hline $72 F m \overline{3} m$ & 3222 & $1\{\{1,0,0\},\{0,1,0\},\{0,0,1\}\}$ & \\
\hline $72 F m \overline{3} m$ & 3222 & $2\{\{1,0,0\},\{1,2,0\},\{1,0,2\}\}$ & \\
\hline $72 F m \overline{3} m$ & $4 \mathrm{mmm}$ & $1\{\{1,0,0\},\{0,1,0\},\{0,0,1\}\}$ & \\
\hline $72 F m \overline{3} m$ & $4 \mathrm{mmm}$ & $2\{\{1,0,0\},\{1,2,0\},\{1,0,2\}\}$ & \\
\hline $72 F m \overline{3} m$ & 523 & $1\{\{1,0,0\},\{0,1,0\},\{0,0,1\}\}$ & \\
\hline $72 F m \overline{3} m$ & $6 m \overline{3}$ & $1\{\{1,0,0\},\{0,1,0\},\{0,0,1\}\}$ & \\
\hline $72 F m \overline{3} m$ & 7432 & $1\{\{1,0,0\},\{0,1,0\},\{0,0,1\}\}$ & \\
\hline $72 F m \overline{3} m$ & $8 \overline{4} 3 m$ & $1\{\{1,0,0\},\{0,1,0\},\{0,0,1\}\}$ & \\
\hline $72 F m \overline{3} m$ & $9 m \overline{3} m$ & $1\{\{1,0,0\},\{0,1,0\},\{0,0,1\}\}$ & \\
\hline $73 \operatorname{Im} \overline{3} m$ & $2 \overline{1}$ & $1\{\{1,0,0\},\{0,1,0\},\{0,0,1\}\}$ & \\
\hline $73 \operatorname{Im} \overline{3} m$ & $2 \overline{1}$ & $2\{\{1,0,0\},\{0,1,0\},\{1,1,2\}\}$ & \\
\hline $73 \operatorname{Im} \overline{3} m$ & $2 \overline{1}$ & $3\{\{2,0,0\},\{0,2,0\},\{0,0,2\}\}$ & \\
\hline $73 \operatorname{Im} \overline{3} m$ & 3222 & $1\{\{1,0,0\},\{0,1,0\},\{3,3,4\}\}$ & \\
\hline $73 \operatorname{Im} \overline{3} m$ & 3222 & $2\{\{1,0,0\},\{0,1,0\},\{1,1,2\}\}$ & \\
\hline $73 \operatorname{Im} \overline{3} m$ & 3222 & $3\{\{1,0,0\},\{0,1,0\},\{0,0,1\}\}$ & \\
\hline $73 \operatorname{Im} \overline{3} m$ & 3222 & $4\{\{1,0,0\},\{0,1,0\},\{3,3,4\}\}$ & \\
\hline $73 \operatorname{Im} \overline{3} m$ & 3222 & $5\{\{1,0,0\},\{0,1,0\},\{1,1,2\}\}$ & \\
\hline $73 \operatorname{Im} \overline{3} m$ & 3222 & $6\{\{1,0,0\},\{0,1,0\},\{0,0,1\}\}$ & \\
\hline $73 \operatorname{Im} \overline{3} m$ & $4 \mathrm{mmm}$ & $1\{\{1,0,0\},\{0,1,0\},\{1,1,2\}\}$ & \\
\hline $73 \operatorname{Im} \overline{3} m$ & $4 \mathrm{mmm}$ & $2\{\{1,0,0\},\{0,1,0\},\{0,0,1\}\}$ & \\
\hline $73 \operatorname{Im} \overline{3} m$ & 523 & $1\{\{1,0,0\},\{0,1,0\},\{3,3,4\}\}$ & \\
\hline $73 \operatorname{Im} \overline{3} m$ & 523 & $2\{\{1,0,0\},\{0,1,0\},\{1,1,2\}\}$ & \\
\hline $73 \operatorname{Im} \overline{3} m$ & 523 & $3\{\{1,0,0\},\{0,1,0\},\{0,0,1\}\}$ & \\
\hline $73 \operatorname{Im} \overline{3} m$ & 523 & $4\{\{1,0,0\},\{0,1,0\},\{3,3,4\}\}$ & \\
\hline
\end{tabular}


Table A.1 - continued from previous page

\begin{tabular}{l|l|l|l} 
ACC & $P_{N}$ & $B_{N}$ matrix row by row & c\# \\
\hline $73 \operatorname{Im} \overline{3} m$ & 523 & $5\{\{1,0,0\},\{0,1,0\},\{1,1,2\}\}$ & \\
$73 \operatorname{Im} \overline{3} m$ & 523 & $6\{\{1,0,0\},\{0,1,0\},\{0,0,1\}\}$ & $1\{\{1,0,0\},\{0,1,0\},\{1,1,2\}\}$ \\
$73 \operatorname{Im} \overline{3} m$ & $6 m \overline{3}$ & $2\{\{1,0,0\},\{0,1,0\},\{0,0,1\}\}$ & $1\{\{1,0,0\},\{0,1,0\},\{1,1,2\}\}$ \\
$73 \operatorname{Im} \overline{3} m$ & $6 m \overline{3}$ & $2\{\{1,0,0\},\{0,1,0\},\{0,0,1\}\}$ & \\
$73 \operatorname{Im} \overline{3} m$ & 7432 & $1\{\{1,0,0\},\{0,1,0\},\{3,3,4\}\}$ \\
$73 \operatorname{Im} \overline{3} m$ & 7432 & $2\{\{1,0,0\},\{0,1,0\},\{1,1,2\}\}$ & \\
$73 \operatorname{Im} \overline{3} m$ & $8 \overline{4} 3 m$ & $\{\{1,0,0\},\{0,1,0\},\{0,0,1\}\}$ \\
$73 \operatorname{Im} \overline{3} m$ & $8 \overline{4} 3 m$ & $4\{1,0,0\},\{0,1,0\},\{3,3,4\}\}$ \\
$73 \operatorname{Im} \overline{3} m$ & $8 \overline{4} 3 m$ & $5\{\{1,0,0\},\{0,1,0\},\{1,1,2\}\}$ \\
$73 \operatorname{Im} \overline{3} m$ & $8 \overline{4} 3 m$ & $6\{\{1,0,0\},\{0,1,0\},\{0,0,1\}\}$ & \\
$73 \operatorname{Im} \overline{3} m$ & $8 \overline{4} 3 m$ & $1\{\{1,0,0\},\{0,1,0\},\{1,1,2\}\}$ & \\
$73 \operatorname{Im} \overline{3} m$ & $8 \overline{4} 3 m$ & $2\{\{1,0,0\},\{0,1,0\},\{0,0,1\}\}$ & \\
$73 \operatorname{Im} \overline{3} m$ & $9 m \overline{3} m$ & & \\
$73 \operatorname{Im} \overline{3} m$ & $9 m \overline{3} m$ &
\end{tabular}


Table A.2 A list of extra conditions referenced in Table A.1. Each entry contains one or more rational polynomial functions that must simultaneously take on integer values. Furthermore, all variables are restricted to non-negative integer values, except for entries 26-29 where $d_{2}$ is further constrained by the inequalities presented and $d_{1}$ can be negative. Some of these conditions have no solutions; some have only a finite number of solutions, some have infinite families of solutions. A discussion of each of the solution space of each condition follows the table.

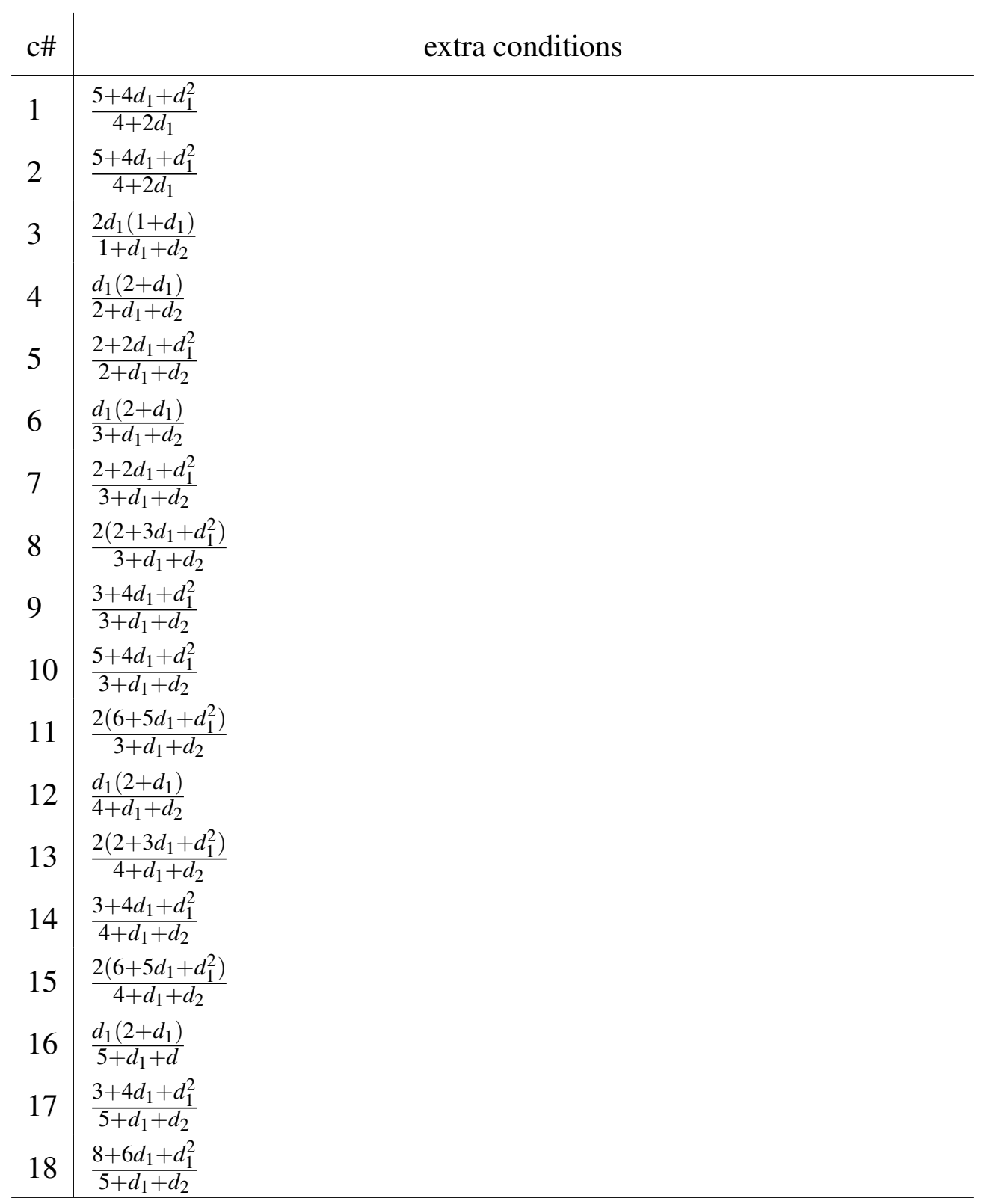


Table A.2 - continued from previous page

\begin{tabular}{|c|c|c|c|}
\hline $\mathrm{c \#}$ & \multicolumn{3}{|c|}{ extra conditions } \\
\hline 19 & $\frac{2 d_{1}\left(1+d_{1}\right)}{3+2 d_{1}+d_{2}}$ & & \\
\hline 20 & $\frac{2\left(2+3 d_{1}+d_{1}^{2}\right)}{4+2 d_{1}+d_{2}}$ & & \\
\hline 21 & $\frac{2\left(2+3 d_{1}+d_{1}^{2}\right)}{5+2 d_{1}+d_{2}}$ & & \\
\hline 22 & $\frac{d_{1}\left(2+d_{1}\right)}{2+d_{1}+d_{2}}$ & $\frac{2+2 d_{1}+d_{1}^{2}}{2+d_{1}+d_{2}}$ & \\
\hline 23 & $\frac{d_{1}\left(2+d_{1}\right)}{3+d_{1}+d}$ & $\frac{2+2 d_{1}+d_{1}^{2}}{3+d_{1}+d_{2}}$ & \\
\hline 24 & $\frac{3+4 d_{1}+d_{1}^{2}}{3+d_{1}+d_{2}}$ & $\frac{5+4 d_{1}+d_{1}^{2}}{3+d_{1}+d_{2}}$ & \\
\hline 25 & $\frac{3+d_{1}}{6+2 d_{1}+3 d_{2}}$ & $\frac{3+d_{1}}{3+d_{1}+d_{2}}$ & \\
\hline 26 & $\frac{d_{1}\left(5+2 d_{1}+3 d_{2}\right)}{4+d_{1}+2 d_{2}}$ & $d_{2} \geq \operatorname{Max}\left[\frac{-1-2 d_{1}}{2}, 0\right]$ & $d_{1}$ can be negative \\
\hline 27 & $\frac{d_{1}\left(7+2 d_{1}+3 d_{2}\right)}{4+d_{1}+2 d_{2}}$ & $d_{2} \geq \operatorname{Max}\left[\frac{-1-2 d_{1}}{2}, 0\right]$ & $d_{1}$ can be negative \\
\hline 28 & $\frac{\left(1+d_{1}\right)\left(4+2 d_{1}+3 d_{2}\right)}{3+d_{1}+2 d_{2}}$ & $d_{2} \geq \operatorname{Max}\left[-d_{1}, 0\right]$ & $d_{1}$ can be negative \\
\hline 29 & $\frac{-2 d_{1}^{2}+d_{2}-d_{1}\left(2+3 d_{2}\right)}{3+d_{1}+2 d_{2}}$ & $d_{2} \geq \operatorname{Max}\left[-d_{1}, 0\right]$ & $d_{1}$ can be negative \\
\hline 30 & $\frac{2 d_{1}\left(1+d_{1}\right)}{1+d_{1}+d_{2}}$ & & \\
\hline 31 & $\frac{1+2 d_{1}+2 d_{1}^{2}}{1+d_{1}+d_{2}}$ & & \\
\hline 32 & $\frac{2 d_{1}\left(1+d_{1}\right)}{2+d_{1}+d_{2}}$ & & \\
\hline 33 & $\frac{d_{1}\left(2+d_{1}\right)}{2+d_{1}+d_{2}}$ & & \\
\hline 34 & $\frac{1+d_{1}+d_{1}^{2}}{2+d_{1}+d_{2}}$ & & \\
\hline 35 & $\frac{2+2 d_{1}+d_{1}^{2}}{2+d_{1}+d_{2}}$ & & \\
\hline 36 & $\frac{3+6 d_{1}+4 d_{1}^{2}}{2\left(2+d_{1}+d_{2}\right)}$ & & \\
\hline 37 & $\frac{2 d_{1}\left(1+d_{1}\right)}{3+d_{1}+d_{2}}$ & & \\
\hline 38 & $\frac{d_{1}\left(2+d_{1}\right)}{3+d_{1}+d_{2}}$ & & \\
\hline 39 & $\frac{2+2 d_{1}+d_{1}^{2}}{3+d_{1}+d_{2}}$ & & \\
\hline 40 & $\frac{2\left(2+3 d_{1}+d_{1}^{2}\right)}{3+d_{1}+d_{2}}$ & & \\
\hline
\end{tabular}


Table A.2 - continued from previous page

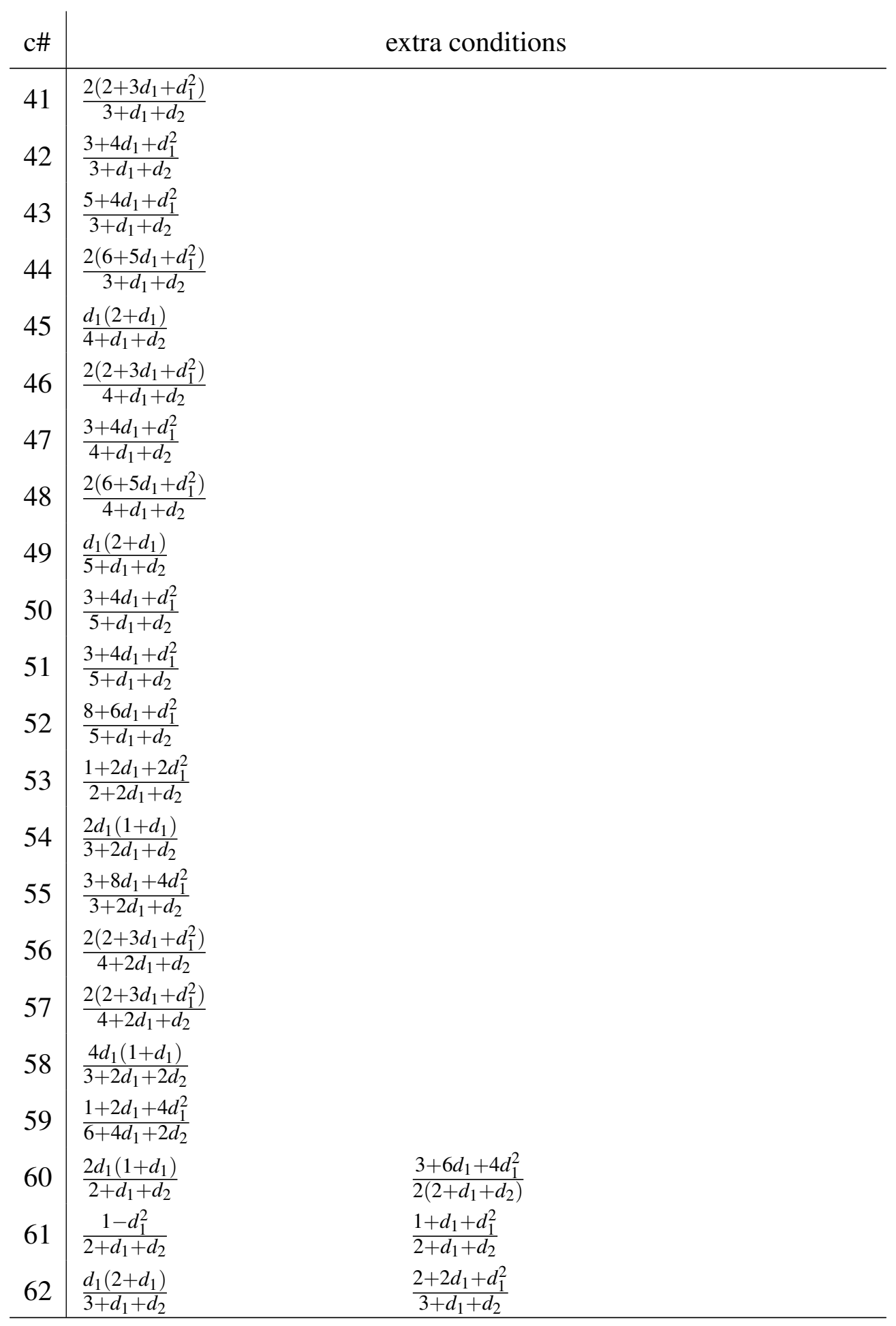


Table A.2 - continued from previous page

\begin{tabular}{l|lll}
$c \#$ & & extra conditions \\
\hline 63 & $\frac{3+4 d_{1}+d_{1}^{2}}{3+d_{1}+d_{2}}$ & $\frac{5+4 d_{1}+d_{1}^{2}}{3+d_{1}+d_{2}}$ \\
64 & $\frac{1+d_{1}^{2}}{3+d_{1}+2 d_{2}}$ & $\frac{1+d_{2}+d_{1}\left(2+d_{2}\right)}{3+d_{1}+2 d_{2}}$ & \\
65 & $\frac{1+d_{1}+d_{1}^{2}}{4+d_{1}+2 d_{2}}$ & $\frac{3+2 d_{2}+2 d_{1}\left(2+d_{2}\right)}{2\left(4+d_{1}+2 d_{2}\right)}$ & \\
66 & $\frac{1-d_{1}^{2}}{1+d_{1}+2 d_{2}}$ & $\frac{\left(-1+d_{1}\right) d_{2}}{1+d_{1}+2 d_{2}}$ & $\frac{\left(1+d_{1}\right)\left(1+d_{2}\right)}{1+d_{1}+2 d_{2}}$ \\
67 & $\frac{2\left(2+d_{1}\right) d_{2}\left(1+d_{2}\right)}{3+d_{1}+2 d_{2}+d_{1} d_{2}+d_{3}}$ & $\frac{2\left(3+d_{1}+2 d_{2}+d_{1} d_{2}+d_{3}\right)}{2+d_{1}}$ & \\
68 & $\frac{\left(1+d_{1}\right)\left(3+8 d_{2}+4 d_{2}^{2}\right)}{4+2 d_{2}+d_{1}\left(3+2 d_{2}\right)+d_{3}}$ & $\frac{4+3 d_{1}+2 d_{2}+2 d_{1} d_{2}+d_{3}}{1+d_{1}}$ & \\
69 & $\frac{2\left(1+d_{1}\right)\left(1+d_{3}\right)}{7+4 d_{3}+d_{2}\left(2+d_{3}\right)+d_{1}\left(3+2 d_{3}\right)}$ & $\frac{2\left(1+d_{1}\right)}{4+2 d_{1}+d_{2}}$ & \\
70 & $\frac{2\left(2+d_{1}\right)\left(1+d_{3}\right)}{8+5 d_{3}+d_{2}\left(2+d_{3}\right)+d_{1}\left(3+2 d_{3}\right)}$ & $\frac{2\left(2+d_{1}\right)}{5+2 d_{1}+d_{2}}$ \\
71 & $\frac{2\left(3+d_{1}\right)\left(1+d_{3}\right)}{9+6 d_{3}+d_{2}\left(2+d_{3}\right)+d_{1}\left(3+2 d_{3}\right)}$ & $\frac{2\left(3+d_{1}\right)}{6+2 d_{1}+d_{2}}$ \\
72 & $\frac{\left(1+d_{1}\right)\left(1+2 d_{3}\right)}{5+4 d_{3}+2 d_{1}\left(1+d_{3}\right)+d_{2}\left(3+2 d_{3}\right)}$ & $\frac{1+d_{1}}{2+d_{1}+d_{2}}$ \\
73 & $\frac{\left(2+d_{1}\right)\left(1+2 d_{3}\right)}{7+6 d_{3}+2 d_{1}\left(1+d_{3}\right)+d_{2}\left(3+2 d_{3}\right)}$ & $\frac{2+d_{1}}{3+d_{1}+d_{2}}$ \\
74 & $\frac{\left(3+d_{1}\right)\left(3+2 d_{3}\right)}{6\left(2+d_{3}\right)+2 d_{1}\left(2+d_{3}\right)+d_{2}\left(5+2 d_{3}\right)}$ & $\frac{3+d_{1}}{3+d_{1}+d_{2}}$ \\
\hline
\end{tabular}

For each c\# that is not specifically listed in the paragraphs below, there are infinitely many solutions. Each of these cases has a single quotient of polynomials of the form $\frac{f\left(d_{1}\right)}{g\left(d_{1}\right)+d_{2}}$, where the polynomial coefficients are all positive integers, and the variables $d_{1}$ and $d_{2}$ must be non-negative integers, and $g\left(d_{1}\right) \neq 0$. Thus, the integer value $k$ of the quotient must be positive. Solving for $d_{2}$ in terms of $k$, we obtain $d_{2}=\frac{f\left(d_{1}\right)}{k}-g\left(d_{1}\right)$. For a given $d_{1}$, every integer value of $k>0$ that divides $f\left(d_{1}\right)$ provides an integer solution for $d_{2}$. For any $d_{1}, k=f\left(d_{1}\right)$ will always be a solution; but there will usually be others as well. Because $d_{1}$ has infinite range, the condition has an infinite number of solutions. 


\section{c\# 1 and 2:}

There are no solutions. To illustrate this, we can expand the quotient as $1+\frac{d_{1}}{2}+\frac{1}{2\left(2+d_{1}\right)}$. Since $\frac{d_{1}}{2}$ is a half-integer and $0<\frac{1}{2\left(2+d_{1}\right)}<\frac{1}{2}$, the sum cannot be integral.

\section{c\# 22:}

There is only one solution. Since both quotients must be integers, it follows that their difference must also be an integer. If we subtract the first quotient from the second, it yields $\frac{2}{2+d_{1}+d_{2}}$ which is only an integer when $d_{1}=d_{2}=0$.

\section{c\# 23, 24, 62, and 63:}

There are no solutions. If we take the difference of the two quotients, we get $\frac{2}{3+d_{1}+d_{2}}$ which must lie strictly between 0 and 1 , and therefore cannot be an integer.

\section{c\# 25:}

There are no solutions. By observation, the second quotient is positive but less than or equal to 1 with equality only when $d_{2}=0$. However, replacing $d_{2}$ with 0 reduces the first quotient to $\frac{1}{2}$ which is clearly not an integer.

\section{c\# 36, 59, 60, and 65:}

There are no solutions. These cases contain a quotient where the numerator is odd while the denominator is even; thus, it can never be an integer.

\section{c\# 58:}

There is one infinite one-dimensional family of solutions. Clearly, $d_{1}=0$ is a solution for any $d_{2} \geq 0$. If $d_{1} \neq 0$, then the numerator of the quotient is even while the denominator is odd, so the 
quotient cannot be integral.

\section{c\# 61:}

There is only one solution. If we take the sum of the two quotients, we get $\frac{2+d_{1}}{2+d_{1}+d_{2}}$ which will only be an integer if $d_{2}=0$. With this in mind we can rewrite the first quotient as $\frac{1-d_{1}^{2}}{2+d_{1}}=$ $2-d_{1}-\frac{3}{2+d_{1}}$ which is only integral if $d_{1}=1$. Thus, the only solution for this case is $d_{1}=1$ and $d_{2}=0$.

\section{c\# 69, 70, 72, and 73:}

There are no solutions. The second quotient in each case has a denominator that is strictly greater than the non-zero numerator and thus it cannot be an integer.

\section{c\# 71 and 74:}

There are no solutions. The second quotient in each case has a denominator that is $d_{2}$ greater than the numerator, so that it is integral if and only if $d_{2}=0$. However, applying $d_{2}=0$ to the first quotient makes its denominator $3+d_{1}$ greater than its numerator, so that the first quotient is not an integer.

\section{c\# 26, 27, 28, 29, 64, 66, 67, and 68:}

There are infinitely many solutions. These cases are more complicated and are still under investigation. 